\begin{abstract}
WOLAK, JUSTYNA EWA. Polyolefin Miscibility: Solid-State NMR Investigation of Phase Behavior in Saturated Hydrocarbon Blends. (Under the direction of Jeffery $L$. White.)

Polyolefin blends represent a vital material field due to their economic and commercial importance. Potential new properties such as lighter weight, lower cost and higher strength, motivate research to investigate blends of saturated hydrocarbon polymers. However, many questions remain concerning how polymer chain structure and packing influence local thermodynamics, or more specifically, the interplay between enthalpy and entropy, which ultimately control bulk phase behavior. Solid-state NMR has proven to be an essential tool in these studies due to its ability to selectively observe molecular level conformations and dynamics without isotopic labeling. Combinations of basic and advanced variable temperature studies such as $1 \mathrm{D}$ and $2 \mathrm{D}{ }^{13} \mathrm{C}$ cross-polarization exchange experiments, static ${ }^{2} \mathrm{H}$ lineshape analysis, ${ }^{1} \mathrm{H}$ relaxation/spin-diffusion measurements, and ${ }^{129} \mathrm{Xe}$ experiments were applied in this work. Several systems were studied, including 50/50 weight percent blends of polyisobutylene with polyethylene-co-1-butene, polyisobutylene with head-to-head polypropylene, and atactic polypropylene with the same polyethylene-co-1-butene samples. The results were used to determine a relationship between miscibility, length scales of mixing, and timescales/length scales of the glass transition.
\end{abstract}


POLYOLEFIN MISCIBILITIY:

SOLID-STATE NMR INVESTIGATION OF PHASE BEHAVIOR

IN SATURATED HYDROCARBON BLENDS

by

JUSTYNA EWA WOLAK

A dissertation submitted to the Graduate Faculty of North Carolina State University

in partial fulfillment of the requirements for the Degree of

Doctor of Philosophy

\section{Chemistry}

Raleigh

2005

APPROVED BY:

Prof. Edward O. Stejskal

Prof. Alex I. Smirnov

Prof. Alan E. Tonelli

Prof. Jeffery L. White

Chair of Advisory Committee 


\section{DEDICATION}

I would like to dedicate this thesis to both my parents. Both of you have supported my goals and have helped and guided me to become the person that I am today. Hopefully one day I can be there for you the way you have for me. 


\section{BIOGRAPHY}

Justyna Ewa Wolak was born on April 10 ${ }^{\text {th }}, 1979$ in Bydgoszcz, Poland to Barbara and Andrzej Wolak. With dreams of becoming an artist, she pursed her piano studies from the age of five and at the age of eight entered the Music Academy in Bydgoszcz, Poland. Life was slightly interrupted when her parents decided to move to Australia, and at the age of ten, she began learning English at Beaconsfield Primary School in Perth, Western Australia. Upon completion of primary school, she was awarded the Dux award from the Minister for Employment, Education and Training, for the highest achievements in Western Australia. She spent a year at Rossmoyne Secondary school specializing in foreign languages and music, where she concentrated on learning Italian and French.

The road did not end in Australia, as in the early nineties, the Wolak family moved permanently to Raleigh, North Carolina where Leesville High School would be the place where the early education would be completed. The decision to attend the University of North Carolina at Chapel Hill prompted the choice to focus studies in chemistry, completing the degree in the year 2001 while also focusing on molecular biology research. The desire to attend graduate school and pursue further chemistry studies led her to North Carolina State University, and the vibrant department of chemistry where she received the graduate student achievement award from GlaxoSmithKline-PLU. While pursing her polymer project with Professor Jeffery L. White, she was part of a collaboration between NCSU and the University of Adam Mickiewicz in Poznan, Poland. Furthermore, a 
successful proposal with an NSF based program called East Asia and Pacific Summer Institute for U.S. Graduate Students, allowed her to learn imaging techniques in Professor Andrew Whittaker's laboratory at the University of Queensland in Brisbane, Australia. Future plans include a post-doc position at Professor Jacob Schaefer's lab at Washington University in Saint Louis, Missouri. 


\section{ACKNOWLEDGMENTS}

One can pay back the loan of gold, but one dies forever in debt to those who are kind. $\sim$ Malayan Proverb

Many people along my journey at NCSU have gone above and beyond their duties to help me with my work and this is my time to thank all of you for your tremendous support. I would like to thank Professor Jeffery L. White for all his hard work, his undivided dedication and his patience in teaching me. Also, I would like to extend a special appreciation to Professor Edward O. Stejskal for his many hours of valuable lessons as well as his patience and help in my numerous presentations. In addition, the experiments were performed smoothly thanks to the aid of Dr. Hanna Gracz, as well as the technical support from the whole electronic shop (Alan, Eddie, Leonard, and Tony). I would like to thank all my fellow group members (Xin Jia, Stan Toporek, Rosimar Rovira and Matthew Truitt) for all their cooperation in sharing the instrument, the helpful discussions and wonderful friendships that grew over the years. Furthermore, I would like to acknowledge and thank my sister Monika and brother Marcin as well as my parents for their continuous help in school work as well as their tremendous support in my life. And last but not least, I would like to thank Jason for all his love and his wonderful friendship. I am deeply grateful to all of you.

Also, the following grants and technical support in this work is gratefully acknowledged. Main support for this project came from the National Science Foundation Grant DMR-0137968 together with additional support from a DuPont 
Science and Engineering Award given to Professor Jeffery L. White. The National Research Council Eastern European Twinning program provided travel support for Professor Jeffery L. White, Justyna Ewa Wolak, Marcin Wachowicz, and Professor Stephan Jurga towards completing the deuterium work. Dr. Tim Shaffer of Exxon Research and Engineering synthesized several labeled polymers, while Dr. David J. Lohse of Exxon Research and Engineering provided other samples and valuable discussion. 


\section{TABLE OF CONTENTS}

LIST OF TABLES Ix

LIST OF FIGURES

CHAPTER 1: Introduction to Polyolefin Blends 1

1-1 Background 2

1-2 Metallocene Catalysis $\quad 7$

1-3 Thermodynamics of Polymer Mixing and Current Methodology 13

1-4 Current Theories for Polyolefin Blend Miscibility 19

1-5 Solid-State Nuclear Magnetic Resonance 27

$1-6$ Objectives 33

CHAPTER 2: Experimental Methods

2-1 Material Characterization 36

2-2 Temperature Calibration of the Bruker MAS Probe 42

2-3 Relaxation and Dipolar Filter Experiments 45

2-4 Cross-Polarization with Magic Angle Spinning (CPMAS) NMR Experiment 52

2-5 Two-Dimensional ${ }^{13} \mathrm{C}$ MAS Exchange NMR Experiment $\quad 58$

2-6 CODEX Description and Experimental Applications 63

2-7 Deuterium NMR Studies and Simulations $\quad 70$

2-8 Xenon NMR Background $\quad 75$

CHAPTER 3: Dynamic Disorder and Conformer Exchange in the Crystalline Monomer of Polycarbonate 82

3-1 Abstract $\quad 83$

3-2 Introduction $\quad 84$

3-3 Experimental 85

3-4 Results and Discussion $\quad 87$

3-5 Conclusions 101

3-6 Supporting Information 102

CHAPTER 4: Polyolefin Miscibility: Solid-State NMR Investigation of Phase Behavior in Saturated Hydrocarbon Blends 103

4-1 Abstract 104

4-2 Introduction 105

4-3 Experimental 107 
4-4 Results and Discussion 109

4-5 Conclusion 124

CHAPTER 5: The Glass Transition Time Scale and Configurational Entropy in Polymers: An Experimental Molecular View

5-1 Abstract

127

5-2 Introduction

127

5-3 Results and Discussion

128

5-4 Conclusions

CHAPTER 6: Length Scales Which Perturb Chain Packing in AmorphousPolymers

6-1 Abstract

6-2 Introduction

136

6-3 Experimental

138

6-4 Results and Discussion

141

6-5 Conclusions

156

CHAPTER 7: CODEX NMR Investigation of Novel Polyolefin Blends

7-1 Introduction

7-2 Different Sources of Atactic Polypropylene

7-3 Miscibility and Dynamics of Atactic Polypropylene Blends

A-1 Pulse Sequence: 2D ${ }^{13} \mathrm{C}-{ }^{13} \mathrm{C}$ Exchange NMR

A-2 Pulse Sequence: Centerband-Only Detection of Exchange (CODEX)

A-3 Supporting Information (.cif file): Bisphenol-A coordinates from $-60^{\circ}$ single- crystal X-ray experiments 


\section{LIST OF TABLES}

Table 1-1 Commercially produced metallocenes (taken from Ref. 4)

12

Table 1-2 Interaction Parameters Determined by SANS

(taken from Ref.11)

Table 2-1 Monomer structures of polyolefins studied

Table 2-2 List of all the polyolefins and their blends investigated

Table 2-3 Selective $\mathrm{T}_{1 \rho}{ }^{\mathrm{H}}$ values on two different systems (taken from Ref. 47)

Table 3-1 X-ray powder data 


\section{LIST OF FIGURES}

\section{CHAPTER 1}

Figure 1-1 Phase diagrams demonstrating (a) UCST, (b) LCST, and (c) UCST plus LCST (taken from Ref. 9).

Figure 1-2 Examples of metallocenes (together with cocatalyst which is not shown) capable of controlling the monomer insertion and making the indicated tacticities of PP: (a) aPP (atactic polypropylene) via a simple metallocene $\mathrm{Cp}_{2} \mathrm{MX}_{2}$ (b) iPP (isotactic polypropylene) via A-bridged (Ind) ${ }_{2} \mathrm{MX}_{2}$ system (typically $\mathrm{A}=\left(-\mathrm{CH}_{2}-\mathrm{CH}_{2}\right)$ and (c) $\mathrm{sPP}$ (syndiotactic polypropylene) via a mixed ligand metallocene of isopropyl-bridged (Cp)(Flu) $\mathrm{MX}_{2}$ (taken from Ref. 2).

Figure 1-3 Estimated annual global demand for SSC-based ethylene polymers (taken from Ref. 4).

12

Figure 1-4 Schematic representing exchange of magnetization via energy conserving flip-flops (taken from Ref. 37)

\section{CHAPTER 2}

Figure 2-1 Several ${ }^{13} \mathrm{C}$ NMR spectra run at $25 \mathrm{MHz}$ of (a) isotactic, (b) atactic, and (c) syndiotactic PP. (The solvent used was 1,2,4-trichlorobenzene at $140^{\circ} \mathrm{C}$ ) (taken from Ref. 40).

Figure 2-2 (a) Top spectrum is a solid-state ${ }^{13} \mathrm{C}$ CPMAS spectrum of aPP demonstrating the broad resonances of the methylene and methyl groups. Much narrower solution resonances can be seen for just the methylene region in (b) representing particular configurations (taken from Ref. 42).

Figure 2-3 Schematic representations of the three different conformations present for the methylene regions in atactic polypropylene. (a) Newman projections of the conformations of a single PP configuration (mrr). As seen in the figure, $\mathrm{g}$ (gauche) conformation marks an interaction involving $\mathrm{CH}_{2}$, while g'(gauche) involves the main-chain $\mathrm{CH}$ and R. (b) A chain schematic showing the same configuration, and the conformations responsible for the three populations in the 
methylene region as effected by the $\gamma$-gauche effect (taken from Ref. 42).

Figure 2-4 (a) Lead nitrate test sample where the center-sample provides the primary temperature measurement, and the bottom part measures the axial temperature gradient as demonstrated in (b) with a large centerband and a small peak from the bottom sample portion (taken from Ref. 44).

Figure 2-5 (a) ${ }^{207} \mathrm{~Pb}$ MAS spectra as a function of time. Spinning sidebands appear at intervals of $2000 \mathrm{~Hz}$ (indicated by $*$ ) and $0 \mathrm{ppm}$ corresponds to $0^{\circ} \mathrm{C}$. (b) Graph showing the expected linear dependence of the chemical shift with temperature.

Figure 2-6 Variable temperature $T_{1 \rho \mathrm{H}}$ plots for the components and the blends. Shown in (a) is aPP with PEE while in (b) aPP with PE-OD and PE-DD (taken from Ref. 35).

Figure 2-7 The dipolar filter pulse sequence where the train of $90^{\circ}$ pulses is followed by the cross-polarization time, and observation with dipolar decoupling. The $\tau$ (spacing in between pulses), $\mathrm{n}$ (number of loops) and mix (mixing time) can all be varied according to the blend requirements (taken from Ref. 33).

51

Figure 2-8 (a) ${ }^{1} \mathrm{H}^{13} \mathrm{C}$ Cross Polarization sequence and (b) thermodynamic energy levels before (top) and (c) after (bottom) the Hartmann-Hahn matching condition (taken from Ref. 38).

55

Figure 2-9 Pulse sequences demonstrating the $2 \mathrm{D}{ }^{13} \mathrm{C}$ MAS exchange experiment with four time periods labeled on top. The $90^{\circ}$ pulses are indicated in black, the cross-polarization (CP) and dipolar decoupling (DD) specified on each channel, and the mixing time $\left(\mathrm{t}_{\mathrm{m}}\right)$ shown as a multiple of the rotor period $\left(\mathrm{t}_{\mathrm{r}}\right)$ (taken from Ref. 9, 38).

Figure 2-10 ${ }^{13} \mathrm{C} \mathrm{CP} / \mathrm{MAS}$ NMR spectra of aPP at (a) $\mathrm{T}=250 \mathrm{~K}$ and (b) $\mathrm{T}=262 \mathrm{~K}$ with spin rate of $3500 \mathrm{~Hz}$. Two-dimensional ${ }^{13} \mathrm{C} \mathrm{CP} / \mathrm{MAS}$ NMR spectra of aPP at $250 \mathrm{~K}$ with mixing times of (c) $500 \mathrm{~ms}$ and (d) $5 \mathrm{~ms}$ (taken from ref. 28, 61)

Figure 2-11 Pulse sequences for CODEX with rotor synchronization indicated on the bottom. The $90^{\circ}$ and $180^{\circ}$ pulses are indicated by black and dashed rectangles, 
respectively (taken from Ref. 64).

Figure 2-12 (a) Three spectra showing the reference, CODEX, and pureexchange for methylmalonic acid at room temperature $\left(\mathrm{t}_{\mathrm{m}}=200 \mathrm{~ms}, \mathrm{t}_{\mathrm{z}}=1 \mathrm{~ms}\right.$ and $\mathrm{Nt}_{\mathrm{r}}=500 \mu \mathrm{s}$ ) (repeated data from S1999 paper to confirm in our pulse program was functional). (b) Differential signal for the pure-exchange of aPMMA (amorphous poly(methyl methacrylate) $)$ indicating slow-exchange taking place $\left(\mathrm{t}_{\mathrm{m}}=500 \mathrm{~ms}\right.$ and $\left.\mathrm{Nt}_{\mathrm{r}}=800 \mu \mathrm{s}\right)$ (taken from Ref. 64, 66).

Figure 2-13 (a) DMS mixed with iPB spinning at $6 \mathrm{kHz}, \mathrm{Nt}_{\mathrm{r}}=667 \mathrm{us}$ and $\mathrm{T}=293 \mathrm{~K}$. (b) iPB at $260 \mathrm{~K}$. Both figures show reference spectra on top and pure-exchange spectra below (taken from Ref. 63).

68

Figure 2-14 Pure CODEX exchange intensities as a function of mixing time for aliphatic quarternary $(\square)$ and protonated aromatic $(\bullet, \bullet, \mathbf{\Delta})$ carbons in BPA (structure above). Data were obtained at $308 \mathrm{~K}$ and using $4 \mathrm{kHz}$ MAS.

Figure 2-15 Quadrupole Echo pulse sequence where the $\pi / 2$ pulses are indicated in white, recovery time is dashed, and $t_{1}$ and $t_{2}$ are the two delays as indicated (taken from Ref. 38).

Figure 2-16 Theoretical ${ }^{2} \mathrm{H}$ NMR lineshapes of several anisotropic motions (taken from Ref. 38).

Figure 2-17 (a) The structure of PPV- $\mathrm{d}_{4}$ with indicated deuteration sites. (b) A static spectrum transitioning to a motionally averaged and axially asymmetric jump spectrum. (c) A variable temperature stackplot of ${ }^{2} \mathrm{H}$ quadrupole echo spectra with the simulations indicated in (d) (taken from Ref. 73).

Figure 2-18 (a) ${ }^{129} \mathrm{Xe}$ NMR spectrum of PP (b) EPDM and (c) blend of PP/EPDM $(80 \% / 20 \%)$ (taken from Ref. 77$)$.

Figure 2-19 Measurements of xenon peaks collapsing vs. differential scanning calorimetric measurements (taken from Ref. 79). 
Figure 2-20 Pulse field gradient Hahn spin-echo (taken from Ref. 38).

Figure 2-21 Graph of $-\ln (\mathrm{A}(G) / \mathrm{A}(G=0))$ vs. $\gamma^{2} \delta^{2}(\Delta-1 / 3 \delta) G^{2}$ where $\mathrm{K}=\gamma^{2} \delta^{2}(\Delta$ $1 / 3 \delta$ ) directly from which diffusion coefficients can be extracted (indicated on the right) (taken from Ref. 38).

\section{CHAPTER 3}

Figure 3-1 $2 \mathrm{D}{ }^{13} \mathrm{C}-{ }^{13} \mathrm{C}$ exchange plot of the BPA aromatic region at $23^{\circ} \mathrm{C}$ using a 1 second exchange time.

Figure 3-2 CP/MAS spectra of $\mathrm{BPA}$ at (a) $-60^{\circ} \mathrm{C}$, (b) $23^{\circ} \mathrm{C}$, (c) $80^{\circ} \mathrm{C}$, and (d) $100^{\circ} \mathrm{C}$. Assignments are indicated on the BPA structure.

Figure 3-3 2D exchange spectra of the aromatic BPA region at (a) $23^{\circ} \mathrm{C}, 0.1 \mathrm{~s}$ exchange; (b) $23^{\circ} \mathrm{C}, 2 \mathrm{~s}$ exchange; (c) $-60^{\circ} \mathrm{C}, 1 \mathrm{~s}$ exchange.

Figure 3-4 Expanded view of the (a) ortho and (b) meta ring carbons of BPA at $23^{\circ} \mathrm{C}$ using a $0.5 \mathrm{~s}$ exchange time. In (a), straight lines are used to indicate the exchange pathways. From these, one observes that only two conformers share multiple correlations.

Figure 3-5 Kinetic plot of the exchange cross peak volume fraction as a function of exchange time $\tau_{\operatorname{mix}}$ for single (-----) and double ( - ) exponential fits. The equations used in the single and double exponential fits are $y=a\left(1-\exp \left(-k_{1} x\right)\right)$, and $y=a\left(1-\exp \left(-k_{1} x\right)\right)+b\left(1-\exp \left(k_{2} x\right)\right)$, respectively.

Figure 3-6 Unit cell structure of BPA, viewed along the a axis, derived from the $23^{\circ} \mathrm{C}$ single crystal $\mathrm{x}$-ray data. The three inequivalent molecular conformations are denoted by color.

Figure 3-7 Potential energy diagram for the gas-phase simulation of ring dynamics in isolated BPA molecules, evaluated at the B3LYP/6-31G* level of theory. Energies of the three low energy equilibrium isomers are shown relative to the lowest energy isomer. Energies of approximate transition state structures, 
determined with QST2 methods at the same level of theory, are shown as barrier heights.

\section{CHAPTER 4}

Figure 4-1 ${ }^{1} \mathrm{H}$ dipolar filter on $\mathrm{PIB} / \mathrm{PEB}-66$ blend at $23^{\circ} \mathrm{C}$ as a function of the number of 12-pulse dipolar filter cycles, in which the interpulse spacing was 10 $\mu \mathrm{s}$. From top to bottom, the number of filter cycles was zero, 10, 20, and 30, respectively.

Figure 4-2 Variable-temeperature CP/MAS stack plot of (a) PIB, (b) PIB/PEB-66 blend, and (c) PIB/PEB-23 blend. Acquisition temperatures are indicated on the figure, and the $\mathrm{PIB} \mathrm{CH}_{2}$ region is expanded in the inset above each spectrum. Note that $\mathrm{PIB} \mathrm{CH}_{2}{ }^{1} \mathrm{H} \mathrm{T}_{1 \rho}$ relaxation time constants at $213 \mathrm{~K}$ are $810 \mu \mathrm{s}, 498 \mu \mathrm{s}$, and $884 \mu \mathrm{s}$ for the samples in (a), (b), and (c), respectively. These results agree with the lineshape data.

Figure 4-3 Selected spectra from variable-temperature static ${ }^{2} \mathrm{H}$ experiments on PIB- $\mathrm{d}_{8}$ (top) and PIB(18 mole\%-d $\mathrm{d}_{8}$ )/PEB-66 blend (bottom) at (a) $210 \mathrm{~K}$, (b) 250 $\mathrm{K}$, (c) $260 \mathrm{~K}$, and (d) $270 \mathrm{~K}$. Although only four data points are shown, spectra were collected at $10 \mathrm{~K}$ increments from $210 \mathrm{~K}$ to $300 \mathrm{~K}$. Note the faster motional timescale of the PIB chains in the blend relative to bulk PIB at the three higher temperatures.

Figure 4-4 Two-dimensional MAS ${ }^{13} \mathrm{C}$ exchange spectra of the PIB methylene region (50-80 ppm) at $213 \mathrm{~K}$ for (a) neat $\mathrm{PIB}$ with 100 millisecond mixing time, (b) neat PIB with 1 second mixing time, (c) PIB/PEB-23 blend with 1.5 second mixing time, and (d) $\mathrm{PIB} / \mathrm{PEB}-66$ blend at $100-\mathrm{ms}$ mixing time. ${ }^{13} \mathrm{C} \mathrm{T}_{1}$ values at $213 \mathrm{~K}$ equal $2.9 \mathrm{~s}$ for the $\mathrm{PIB} \mathrm{CH}_{2}$ carbons in all samples.

Figure 4-5 Two-dimensional MAS ${ }^{13} \mathrm{C}$ exchange spectra of the PIB methylene region (50-80 ppm) at $213 \mathrm{~K}$ for a PIB/PEB-66 blend in which the PIB contains $5 \%{ }^{13} \mathrm{C}$-labeled $\mathrm{CH}_{2}$ groups at mixing times of (a) $5 \mathrm{~ms}$, (b) $25 \mathrm{~ms}$, (c) $50 \mathrm{~ms}$, (d) $100 \mathrm{~ms}$, and (e) 1 second.

Figure 4-6 Static ${ }^{129} \mathrm{Xe}$ NMR spectra of $\mathrm{Xe}$ gas adsorbed in (a) PIB, (b) PIB/PEB-66 blend, (c) PEB-66, (d) PIB, (e) PIB/PEB-23 blend, and (f) PEB-23. 
The number of transients vary in each case, but are greater than $1 \mathrm{~K}$ for all. The mass of each sample also varies between experiments.

\section{CHAPTER 5}

Figure 5-1 Comparison of DSC $\mathrm{T}_{\mathrm{g}}$ trace obtained with a $2 \mathrm{~K} / \mathrm{min}$ scan rate (top) with $2 \mathrm{D}{ }^{13} \mathrm{C}$ exchange spectra at (a) $203 \mathrm{~K}$ and $1 \mathrm{~s}$ mix time; (b) same as (a) at $2 \mathrm{~s}$ mix; (c) same as (a) at $4 \mathrm{~s}$ mix; (d) $208 \mathrm{~K}$ at $1 \mathrm{~s}$ mix time; (e) same as (d) at $2 \mathrm{~s}$ mix; (f) same as (d) at $4 \mathrm{~s}$ mix. Spinning speeds were $4 \mathrm{kHz}$.

Figure 5-2 2D exchange spectrum at $208 \mathrm{~K}$ of (a) methylene carbons in pure PIB with a 2 s mixing time; (b) methylene carbons of PIB in a 50:50 blend with PEB using a $100 \mathrm{~ms}$ mixing time. Slices through the 65-ppm trans-gauche peak are shown on the top axis, and within the signal-to-noise limits, exhibit the same features.

\section{CHAPTER 6}

Figure 6-1 Static ${ }^{2} \mathrm{H}$ NMR spectra for $80 \%-\mathrm{PIB}-\mathrm{d}_{8}$ versus temperature, showing a glass transition on the ${ }^{2} \mathrm{H}$ NMR timescale at $\approx 265 \mathrm{~K}$.

Figure 6-2 Comparison of static ${ }^{2} \mathrm{H}$ NMR spectra over the $\mathrm{NMR} \mathrm{T}_{\mathrm{g}}$ range for 20\%-PIB- $\mathrm{d}_{8}$ chains in (a) a blend with PEB-66; (b) a blend with PEB23; (c) bulk, i.e., pure $20 \%$-PIB- $\mathrm{d}_{8}$. Note the difference in the spectral coalescence points in both (a) and (b), relative to (c), which is even more clear by comparing the lineshapes across the second row.

Figure 6-3 Top schematic shows a simulated conformer of an (a) all trans chain segment with introduction of a single trans-gauche (gauche + or gauche -) exchange event in (b). Bottom schematic shows conformational dynamics model used to simulate the motionally-averaged $\mathrm{CD}_{3}$ powder patterns. Individual methyl groups are denoted as "A" and "B".

146

Figure 6-4 Comparison of (a) experimental and (b) simulated variabletemperature ${ }^{2} \mathrm{H}$ spectra for the $20 \%-$ PIB- $_{8}$ blended with PEB-23. 
Figure 6-5 (a) Arrhenius plot of chain reorientation rates obtained via simulation of the experimental static ${ }^{2} \mathrm{H}$ lineshapes using the four-site model described in the text for $20 \%$-PIB- $\mathrm{d}_{8}$ chains in each of the indicated environments. The dotted lines indicate the region near lineshape collapse. (b) Relaxation rate data vs. temperature for ${ }^{2} \mathrm{H} \mathrm{T}_{2}$ experiments. Note that the same activation energy is obtained via lineshape simulation (a) and in the linear region of the $\mathrm{T}_{2}$ curve (b).

Figure 6-6 Static ${ }^{129} \mathrm{Xe}$ NMR spectra for xenon gas absorbed in (a) pure PIB vs. blends with decreasing 1-butene comonomer amounts: (b) 50:50 PIB/PEB-97 blend; (c) 50:50 PIB/PEB-66 blend; (d) 50:50 PIB/PEB-23 blend; (e) pure PEB97; (f) pure isotactic poly-1-butene. The free xenon peak at $0 \mathrm{ppm}$ is not shown for clarity, and lower sample masses were used in 6-6(c) and 6-6(e).

Figure 6-7 Comparison of two non-invasive experimental methods of miscibility for a polyolefin blend: (a) static ${ }^{129} \mathrm{Xe}$ spectra of a $\mathrm{PIB} / \mathrm{hhPP}$ blends vs. its pure components; (b) ${ }^{1} \mathrm{H}$ solid-state spin diffusion data for the same blend as in (a). Note that the slopes of all increasing and decreasing curves in (b) are of similar absolute magnitude, indicating that polarization transfer between PIB and hhPP chains occurs with a timescale similar to spin-diffusion within PIB chains, proving intimate mixing (taken from Ref. 33 \&138).

Figure 6-8 ${ }^{129} \mathrm{Xe}$ PFG experimental results for pure PIB (•) and PIB/PEB-66 (o), yielding diffusion coefficients of $2.9 \times 10^{-8} \mathrm{~cm}^{2} / \mathrm{s}$ and $2.0 \times 10^{-7} \mathrm{~cm}^{2} / \mathrm{s}$, respectively.

Figure 6-9 Schematic of mixing length scales derived from ${ }^{129} \mathrm{Xe}$ NMR data in (a) the PIB/PEB-23 blend. For the PIB/PEB-66 blend, either (b) or (c) are possible representations, since the average domain size is $<70 \mathrm{~nm}$ for each.

155

\section{CHAPTER 7}

Figure 7-1 Variable temperature cross-polarization studies of two samples of (a) aPP(Eastman) and (b) aPP(PolySciences). 
Figure 7-2 Solution spectra taken at $360^{\circ} \mathrm{C}$ for (a) aPP(PolySciences) and (b) aPP(Eastman), which were dissolved in 1,1,2,2-tetrachloroethane- $\mathrm{d}_{2}$.

Figure 7-3 Static ${ }^{129}$ Xe NMR spectra for xenon gas absorbed in (a) pure aPP versus blends with increasing 1-butene comonomer amounts: (b) 50:50 aPP/PEB23.5; (c) 50:50 aPP/PEB-66; (d) 50:50 aPP/PEB-86; (e) 50:50 aPP/PEB97; (f) pure isotactic poly-1-butene.

Figure 7-4 Static ${ }^{129}$ Xe NMR spectra for xenon gas absorbed in PEB copolymers with the following varying 1-butene comonomer concentrations (in weight \%): (a) PEB-23.5; (b) PEB-66; (c) PEB-86; (d) PEB-97; (e) pure isotactic poly-1-butene.

Figure 7-5 A comparison of the chemical shift differences between (a) PIB/PEB66 versus (b) aPP/PEB-66 via cross-polarization plots performed at $213 \mathrm{~K}$.

Figure 7-6 Three spectra of aPP at $260 \mathrm{~K}$ are displayed indicating how the pureCODEX spectrum is obtained. The pure-CODEX spectra signal was magnified by two.

166

Figure 7-7 Pure-CODEX spectra for (a) pure aPP, (b) aPP/PEB-66 and (c) PEB66. All represent either $100 \mathrm{~ms}$ or $200 \mathrm{~ms}$ exchange times acquired with $12 \mathrm{~K}$ scans and line-broadening of $75 \mathrm{~Hz}$.

Figure 7-8 Variable temperature cross-polarization stackplots comparing the (a) pure PIB versus (b) PIBhhPP $\left(5 \%{ }^{13} \mathrm{C}\right.$ labeled) and (c) hhPP.

Figure 7-9 Pure-CODEX spectra for (a) PIB, (b) PIB/hhPP, and (c) hhPP at various temperatures as indicated. All spectra were run at a mixing time of $100 \mathrm{~ms}$ and $12 \mathrm{~K}$. All the spectra at $260 \mathrm{~K}$ were magnified by two, while the rest were plotted on the same scale. 
Chapter 1: Introduction to Polyolefin Blends 


\section{1-1 Background}

Heterogeneous materials ranging from thermoplastic elastomers to multicomponent plasticized blends, are becoming widespread in academia and industry. ${ }^{1}$ The promising polyolefin field offers enhanced physical properties to address economic issues and potential answers to fundamental, scientific questions. Extensive applications of polyolefins are abundant in areas such as kitchen materials (disposable food-packaging, dishwasher-safe containers and grocery bags), household supplies (toys, and indoor-outdoor carpeting), and industrial/work equipment (tires, bullet proof vests, and aerospace structural designs). ${ }^{2}$ Typically, polyolefins are produced and used as blends offering attractive properties such as light weight, low cost, high strength, high chemical resistance and low dielectric constants. ${ }^{1,2}$ The 50 million tons of annual polyolefin production reveals the demand of today's society as well as the need to make these polymers economically accessible. Recent advances in metallocene catalysis have allowed new, previously inaccessible, polyolefins to be available with resulting increases in the various blend combinations. ${ }^{3,4}$ The polyolefin field can now reap the benefits of metallocene catalysis which offers advantages over the usual Ziegler-Natta polymerization, such as controlled monomer incorporation, specific tacticities with narrow and higher molecular weight and sequence distributions. ${ }^{5-7}$ Advances brought about by metallocene catalysis have rekindled interests to fully characterize polyolefin miscibility and their phase behaviors, because our current predictive capabilities of blend behaviors are 
limited, due in large measure to the lack of experimental data at the microscopic or chain level.

Currently, commercial applications of polyolefin blends are found in both homogeneous and heterogeneous materials. ${ }^{8}$ Homogenous or miscible means that the blend is intimately mixed on the molecular level, whereas a heterogeneous or immiscible blend possesses phase separation. ${ }^{9}$ The phase diagrams in Figure 1-1 can be helpful in visualizing how miscibility for a pair of polymers is dependent on what composition is chosen, as well as the temperature. In (a), the solid line shows where the blend becomes immiscible and as indicated, this is called the upper critical solution temperature (UCST). Part (b) reveals what happens for a blend displaying a lower solution critical temperature (LCST), whereas (c) illustrates a blend having both UCST and LCST. Many polymers that are compatible with one another are near phase boundaries for the particular

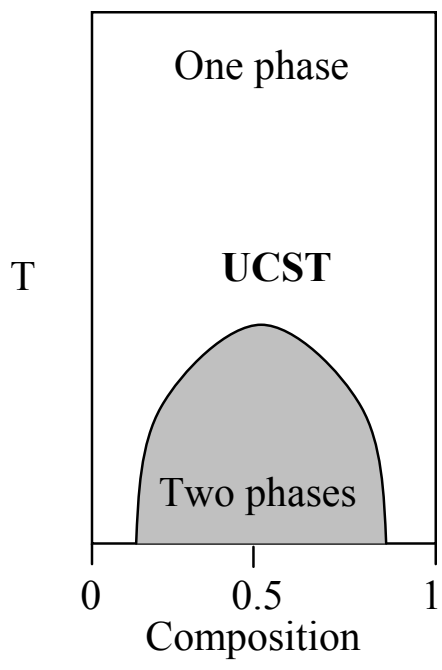

(a)

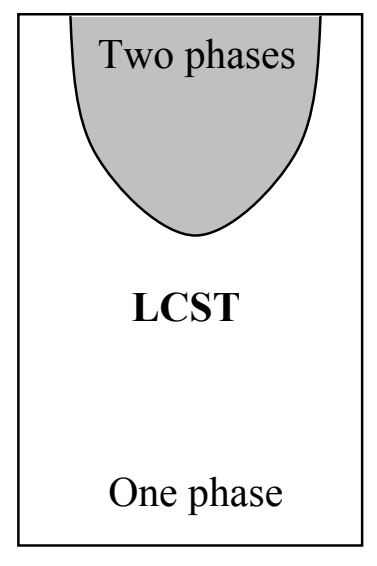

(b)

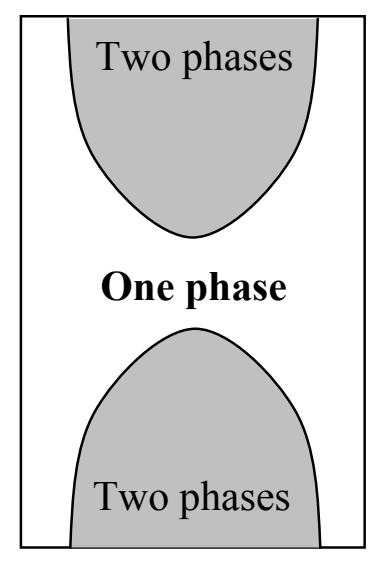

(c)

Figure 1-1 Phase diagrams demonstrating (a) UCST, (b) LCST, and (c) UCST plus LCST (taken from Ref.9). 
molecular weights and compositions used. In industry, certain immiscible blends are considered compatible if the blend is useful, i.e., the heterogeneity is not on a large enough scale to effect its application. The key to the usefulness of polyolefin blends lies in knowing and understanding the level at which they are mixed. Once the miscibility level is established, a precise prediction of the material behavior under various conditions and numerous applications can be made.

\section{Challenges of Studying Polyolefin Blends}

Polyolefins are difficult materials to study due to their chemically similar structures. They do not obey "like dissolves like" solubility conventions. One would imagine that all these saturated hydrocarbons should be miscible with one another. Alternatively, the lack of polarity or functionality leads to no specific attractions between the chains and one might predict no mixing at the molecular level. Nevertheless, miscible systems are encountered, but the state of the field is unable to offer an explanation for the factors influencing mixing. For example, at this time, we cannot explain why polyethylene and polypropylene do not mix with each other at the chain level, but upon altering the polyethylene chain with certain sidegroups, the two exhibit a miscibility window. ${ }^{1}$ Bates et al. reported that our knowledge of polyolefin thermodynamics is 'primitive' even though their interactions are simple, yet "...it is just this chemical similarity that leads to a variety of complications in attempting to model the mixing thermodynamics of

polyolefins." 10 Being able to control polyolefin properties by determining miscibility windows makes 'engineering' polymer blends with desirable behavior, 
such as increased toughness and larger temperature ranges, an attainable goal in the future. ${ }^{11-13}$

Current investigations are exploring how factors such as polymeric microstructure, tacticity, comonomer incorporation, and chain dynamics all contribute in determining local structure and bulk morphology for polyolefin blends. ${ }^{14-17}$ Questions on how these play a part in the enthalpy and entropy of these systems, which according to thermodynamics (Flory-Huggins theory) determine bulk phase behavior in the end, have only started to be answered. Many groups are attempting to map out these complex behaviors by suggesting phase diagrams and miscibility windows for obtaining miscible blends. What makes polyolefins such a challenging field is their chemical and physical similarities, e.g. essentially identical refractive indecies, melt densities and glass transitions, so typical characterization methods, such as differential scanning calorimetry and cloud points, are often uninformative. Small-angle neutron scattering (SANS) has been responsible for most of the advances in polyolefin blend studies, where direct information on the thermodynamic interactions of the single-phase mixtures was obtained. A drawback of this technique is that one of the polymers has to be deuterium-labeled, and thus the isotopic effect has to be considered. ${ }^{1}$

Various approaches have been taken to explain non-ideal polyolefin blend mixing, such as solubility parameters, conformational asymmetry theory, lattice cluster theory, and the polymer reactive interactive site model (PRISM) ${ }^{10}$ Many groups have studied these blends using a variety of experimental and theoretical techniques, but magnetic resonance data has been absent from this literature. 


\section{Solid-State NMR and Systems Studied}

Solid-state NMR (SSNMR) is uniquely suited to probe the fundamental phase behavior or polyolefin miscibility, where 'mixing' is required for fabrication of new polymers, but also for separation via reclamation and recycling. SSNMR has proven to be an essential tool in studying polyolefin chain level mixing due to its ability to selectively observe molecular level conformers, dynamic rates and maximum/minimum length scales without isotopic labeling. ${ }^{13}$,

${ }^{18}$ Due to its noninvasive capabilities, SSNMR is an inexpensive technique with direct industrial application as opposed to previously used techniques such as small-angle neutron scattering. 11, 12, 14, 15, 19 An assortment of experiments are applied. For instance, one and two-dimensional ${ }^{13} \mathrm{C}$ cross-polarization exchange experiments, static ${ }^{2} \mathrm{H}$ lineshape analysis, ${ }^{1} \mathrm{H}$ relaxation measurements and ${ }^{129} \mathrm{Xe}$ NMR. ${ }^{13}$ Detailed microscopic to mesoscopic (i.e. angstroms to ten of nanometers) data has been acquired on blend systems such as PIB (polyisobutylene) and PEB (polyethylene-co-1-butene) with varying butene composition, PIB and aPP (atactic polypropylene), PIB and iPP (isotactic polypropylene), and lastly, aPP and PEB.

Major research efforts have been concentrated on blends involving polyisobutylene (PIB) and the copolymer polyethylene-co-butene (PEBs) as the other component, which exhibit anomalous interaction parameters. The developed experimental approaches have been key in revealing local packing, dynamics, and length scales of mixing in several blends resulting in a miscibility window for PIB with several PEBs. Our group was the first to provide molecular/chain level 
evidence suggesting that configurational entropy is a driving force for miscibility in these polyolefin blends. Simply put, the Flory-Huggins theory only considers combinatorial entropy in its equation, and disregards the possible existence of configurational entropy. Traditional theories of macromolecular mixing simply do not treat the polymer at the local chain level and until this work, configurational entropy has been overlooked as a possible contribution to polymer blending. Acquisition and understanding of local microscopic data, which directly determines macroscopic properties, should enable the synthesis of new blends with desired properties and aid in determining the specific chain packing and chain architecture contributions to phase behavior.

\section{1-2 Metallocene Catalysis}

The Ziegler-Natta (ZN) polymerization discovered in the 1950s was the first method which allowed for stereochemical control of the olefin polymerization process. ${ }^{3}, 4,20$ The 'multi-site' ZN catalysts have several drawbacks: for example, inability to polymerize all monomers, as well as their non-uniform active centers, resulting in broad molecular weight distributions (MWD). ${ }^{5}$ These issues were solved upon the discovery of metallocene catalysis polymerization in the $1970 \mathrm{~s},{ }^{3,4}, 20$ where these novel catalysts gave not only higher activity and stereospecificity, but their 'supported' versions have simplified polymerization and transformed their commercial production. ${ }^{20}$

The metallocene catalyst system consists of a metallocene and a cocatalyst where the term metallocene comes from 'the iron sandwich' (ferrocene), 
composed of an iron atom in between planar cyclopentadienyl $(\mathrm{Cp})$ groups. $^{2}$ Today, the most active metallocenes are composed of substituted $\mathrm{Cp}\left(\mathrm{Cp}=\eta^{5}\right.$ $\mathrm{C}_{5} \mathrm{H}_{5}$ ), indenyl (Ind) and fluorenyl (Flu) ligands with either $\mathrm{Zr}$, Ti, or $\mathrm{Hf}$ as metal centers, where the active center is shielded from the immediate electronic environment. ${ }^{3}$ The discovery of methylaluminoxane (MAO) as the cocatalyst, by Walter Kaminsky's group in the 1970s, revived commercial interest in metallocenes due to the large increase in activity achieved., ${ }^{3,5}$ The resulting combination of the various available metallocenes (titanocenes, zirconocenes, or hafnocenes) with their cocatalysts, produce extremely active polymerization catalysts as an example, for ethylene polymerization, the zirconocene/MAO catalysts are 10-100 times more active than conventional Ziegler catalysts. ${ }^{21}$ Not only are the metallocenes more active then Ziegler-Natta $(\mathrm{ZN})$ catalysts, but they are also cost competitive in polyolefin production. ${ }^{22}$

Even though higher activity and cost effectiveness are very important, even more noteworthy are the novel polymers and assorted properties obtainable only via metallocene catalysis. ${ }^{22}$ Several combinations of the catalyst/cocatalyst components play vital roles in determining the activity, molecular weight, comonomer incorporation and stereospecificity. ${ }^{5}$ An assortment of ligands and their substituents, the presence/absence of bridges, metals and cocatalysts, play important roles in polymerizing a polymer with specific properties. ${ }^{3}$ A chief advantage of metallocene catalysts is the fact that they have single active sites, which are essentially identical, allowing for polymerization of very uniform polymers. ${ }^{2}$ The ability to control the catalysts's stereochemistry allows for the 


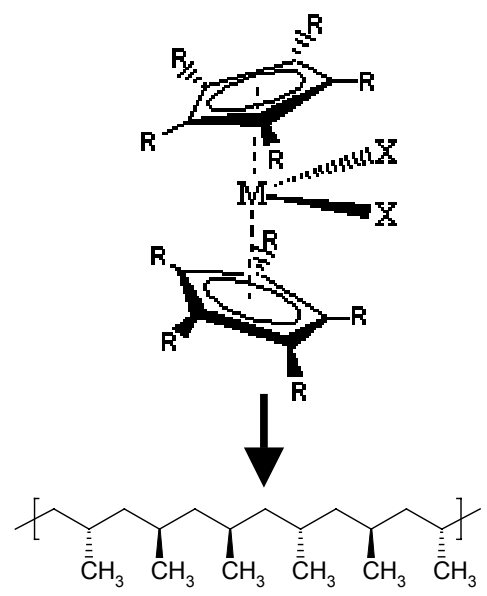

(a)

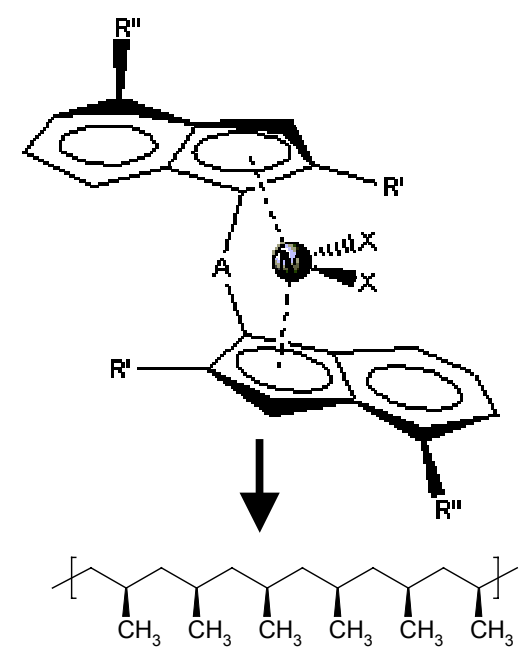

(b)

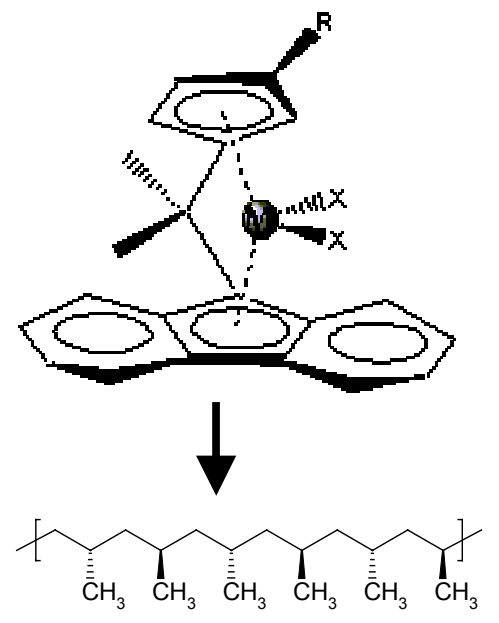

(c)

Figure 1-2 Examples of metallocenes (together with cocatalyst which is not shown) capable of controlling the monomer insertion and making the indicated tacticities of PP: (a) aPP (atactic polypropylene) via a simple metallocene $\mathrm{Cp}_{2} \mathrm{MX}_{2}$ (b) iPP (isotactic polypropylene) via A-bridged (Ind) ${ }_{2} \mathrm{MX}_{2}$ system (typically $\mathrm{A}=\left(-\mathrm{CH}_{2}-\mathrm{CH}_{2}\right)$ and (c) $\mathrm{sPP}$ (syndiotactic polypropylene) via a mixed ligand metallocene of isopropyl-bridged (Cp)(Flu) $\mathrm{MX}_{2}$ (taken from Ref.2).

production of extremely stereospecific polymers via sterically crowded catalyst complexes as indicated in Figure 1-2. For instance, the simple, unrestricted metallocene in Figure 1-2(a) gives a mixture of monomer insertions, whereas the bulkier, constrained metallocenes in (b) and (c) give very specific tacticities. ${ }^{2}$ The diverse tacticities are key in determining polymer's properties, for example, 
atactic polypropylene is soft, sticky, and weak while isotactic polypropylene can crystallize and make fibers used in ropes and webbing. ${ }^{23}$

\section{Commercialization of Metallocene Catalysts}

Since the introduction of commercial, metallocene-made polyolefins in 1991, new polyolefin structures with novel applications have surfaced. The early prediction of 'metallocenes having a very bright future in plastics' has been realized with the capability to almost 'engineer' polymer structure and properties 'as if each metallocene holds the genetic code for a specific polymer family'. Although the expansion of metallocene catalysts has been rapid, Ziegler-Natta (ZN) catalysts still control worldwide polyethylene (PE) and polypropylene (PP) industrial production and the metallocene field has been reclassified from 'mature' in the 1990 s to a 'growth business' today. ${ }^{4}$ At the same time new opportunities, expanding from the old $\mathrm{ZN}$-material performance, such as demanding exact physical properties, are potential goals in this expanding metallocene field. ${ }^{22}$

Fundamental challenges in early commercialization of metallocene catalysts were improving the required cocatalyst ratio to effectively lower the production costs and discovering practical operating conditions. At the beginning, no support was used causing high cocatalyst ratios resulting in expensive systems with high catalyst residues in the final polymers. The solution was found in fluoroaryl-based cocatalysts, which brought down the expenses of using a large

excess of aluminoxane. ${ }^{5,21}$ The cocatalysts' were commercially 'friendly' because they still enabled the formation of a metallocenium cation, provided charge 
stabilization and weak coordination without reaction with the highly reactive metallocene cation, while they brought down the amount of cocatalyst needed. For example, in order to have an active catalyst, the ratio of MAO to metallocene needs to be $5000: 1$ whereas this ratio is $1: 1$ for borate to metallocene. ${ }^{2}$ In order to be adapted to industrial processes (e.g. slurry or gas-phase), the homogeneous metallocene catalysts had to be heterogenized. The solution came with supported catalysts addressing factors such as activity, reduced amounts of MAO, and higher molecular weights ${ }^{24}$ in order to achieve similar/improved polyolefins compared to their homogeneous equivalents. The supports play an important role in the polymerization processes of supplying the catalyst to the reactor and serving as templates while at the same time being highly productive and cost efficient. $^{21}$

Over the past decade, the search for new polymerization-stable ligands has stimulated 'non-metallocene' single-site catalysts (SSC's) for olefin polymerization. ${ }^{5}$ Some major SSCs (single-site catalysts) companies that are already commercially producing products, or soon will be, are Basell, Chevron Phillips Chemical, Dow Chemical, DuPont Dow Elastomers, Exxon Mobil Chemical, Mitsui Chemicals, NOVA Chemicals, and Titan Polyethylene etc. ${ }^{4}$ Table 1-1 describes some of the SSC-based polymers that are already commercially available. Commercialization has been slow for some of these plastics, since it often takes years to establish new markets. The metallocene/SSCs have produced new specialty polymers such as thermoplastics (high-temperature resistance) and high performance elastomers, replaced 
Table 1-1 Commercially produced metallocenes(taken from Ref. 4)

\begin{tabular}{|l|l|}
\hline SSC-based product & Application/markets \\
\hline LLDPE & $\begin{array}{l}\text { Cast and blown film (often multiplayer), } \\
\text { extrusion coating, shrink film }\end{array}$ \\
\hline $\begin{array}{l}\text { HDPE and MDPE }(0.935 \text { to } \\
0.960 \mathrm{~g} / \mathrm{ml})\end{array}$ & $\begin{array}{l}\text { Rotomolding, injection molding, blow } \\
\text { molding, film }\end{array}$ \\
\hline $\begin{array}{l}\text { Ethylene- } \alpha \text {-olefin plastomers } \\
(0.880-0.910 \mathrm{~g} / \mathrm{ml})\end{array}$ & $\begin{array}{l}\text { PP modification, controlled permeability, } \\
\text { packaging films }\end{array}$ \\
\hline $\begin{array}{l}\text { Ethylene elastomers (ethylene- } \\
\text { octene, ethylene-butene) }\end{array}$ & $\begin{array}{l}\text { TPOs and TPVs, particularly for automotive } \\
\text { markets }\end{array}$ \\
\hline $\begin{array}{l}\text { Isotactic PP homo-, random-, and } \\
\text { impact-copolymers }\end{array}$ & $\begin{array}{l}\text { Injection molding, non-woven fabrics, fibers, } \\
\text { films, automotive interiors }\end{array}$ \\
\hline Syndiotactic PP copolymers & $\begin{array}{l}\text { Shrink films for labels, injection moldings, } \\
\text { adhesives, sheet extrusion }\end{array}$ \\
\hline Syndiotactic polystyrene & $\begin{array}{l}\text { Engineering plastic competing with nylons, } \\
\text { PBT, acetals, PPS and other ETPs }\end{array}$ \\
\hline
\end{tabular}

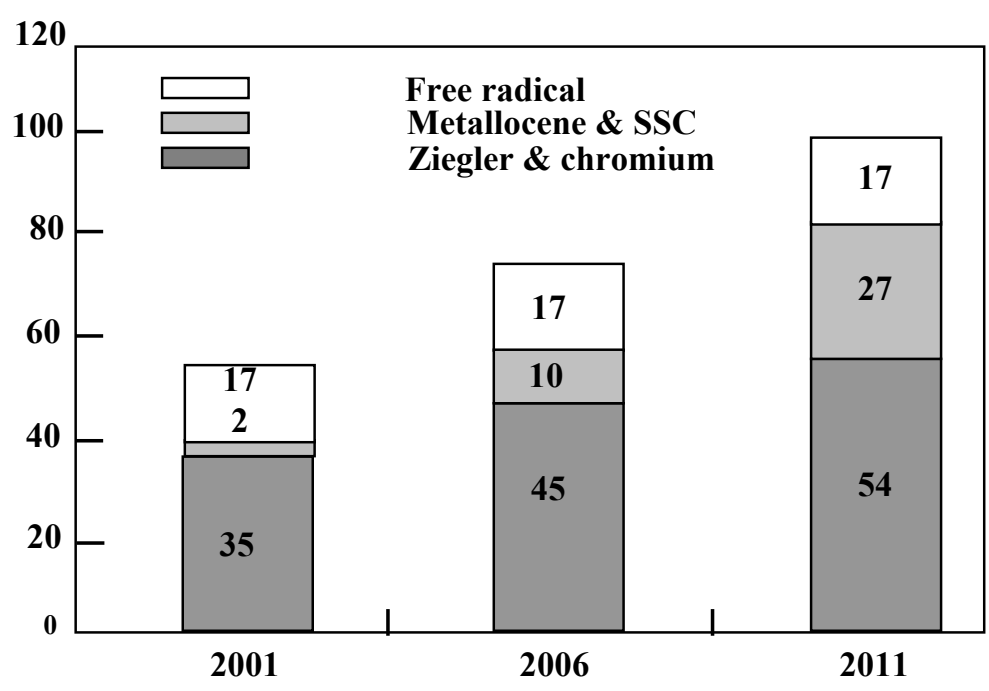

Figure 1-3 Estimated annual global demand for SSC-based ethylene polymers (taken from Ref. 4).

conventional catalysts, expanded markets to applications previously inaccessible, and created new fields for polyolefins at the expense of other higher-cost non polyolefin materials. The estimated projections for PE demand in the coming years are presented in Figure 1-3. ${ }^{2}$ Widespread demand of the various polyolefins can be found in film packaging, adhesives, foam products, cable insulation, and 
environmentally-friendly replacements of other plastics. ${ }^{3}$ As demonstrated, the SSC-based PE demand has large expectations for the coming years.

\section{1-3 Thermodynamics of Polymer Mixing and Current Methodology}

\section{Flory-Huggins Theory for Macromolecules}

Miscibility in macromolecules may be described by the Flory-Huggins theory of the free energy of mixing polymers in Equation 1-1. ${ }^{1}$ The first two terms are the combinatorial entropy term defined as all the different ways that the chains are randomly packed on a two-dimensional lattice. The third term is the enthalpy term that includes all the interactions between the polymer segments such as hydrogen bonding, weak Van der Waals forces, or electron donor-

$$
\frac{\Delta G}{R T}=\frac{\phi_{1}}{v_{1} N_{1}} \ln \phi_{1}+\frac{\phi_{2}}{v_{2} N_{2}} \ln \phi_{2}+\phi_{1} \phi_{2} \frac{\chi}{\sqrt{v_{1} v_{2}}}
$$

acceptor pairs. The symbols in Equation 1-1 are the following: $\phi_{\mathrm{i}}$ (volume fraction of the component), $v_{\mathrm{i}}$ (molar volume of the component), $\mathrm{N}_{\mathrm{i}}$ (degree of polymerization of component), $\left(v_{1} v_{2}\right)^{1 / 2}$ root mean square molar volume (or can also be expressed as $v$ ), $\chi$ (interaction parameter) and $\mathrm{R}$ (universal gas constant). Equation 1-2 shows the definition of the "Flory-Huggins interaction parameter" that is introduced into the main equation by the enthalpy term. ${ }^{1}$ The terms in Equation 1-2 are z (coordination number), $\Delta \varepsilon$ (energy required to form segmentsolvent contacts), $\mathrm{n}_{\mathrm{i}}$ (molecules), $\mathrm{k}$ (Boltzmann constant) and $\mathrm{T}$ (temperature in Kelvin). 


$$
\begin{gathered}
H_{m}=z \Delta \varepsilon n_{1} \phi_{2}=k T \chi n_{1} \phi_{2} \\
\text { where } \chi=\frac{z \Delta \varepsilon}{k T}
\end{gathered}
$$

The derivation of the Flory-Huggins equation describing the Gibbs free energy of mixing is based on the liquid-lattice theory, but as seen above, it does not contain any lattice variables. ${ }^{9}$ There are certain drawbacks and approximations present in this equation that have encouraged researchers to improve it. For example the model assumes no volume change with mixing, excludes possible self-interactions of the chains, and addresses only combinatorial entropy while ignoring possible local configurational contributions. ${ }^{25,26}$ It defines each monomer in the polymer simply by the size of the lattice point assuming that the local structure and local dynamics will be the same for all polymer types. Other approximations, such as the assumption of uniform placement of polymer segments on the lattice, require high concentrations and deviates when polymer solutions are dilute. Lastly, the Flory interaction parameter should have both enthalpic and entropic contributions, and also seems to depend on concentration. ${ }^{25}$ Yet, even though these apparent deficiencies are present, the Flory-Huggins theory has been the foundation in polymer thermodynamics since the 1940 s, and has successfully explained a large volume of experimental data. ${ }^{25,26}$

With polymer material applications becoming widespread, researchers are focusing on expanding the current knowledge pertaining to exact levels of mixing. ${ }^{9}$ As observed from the Flory-Huggins equation, polymer blends are 
typically immiscible because as molecular weight increases, the entropy of mixing decreases making this term negligible. Therefore, according to thermodynamics, when miscibility is encountered, it is the negative interaction parameter that is responsible for mixing. Stated differently, the interactions in the blend are more favorable or less unfavorable as compared with the interactions experienced by the polymer chains within the pure components. ${ }^{9}$ In general, it is considered that compatibility between blends can be associated with favorable specific chemical interactions between the blend components.

For polyolefin blends, this raises questions because the lack of polar or functional groups points towards no specific interactions between the different polymer chains. ${ }^{1}$ The intermolecular energies of saturated hydrocarbon liquids originate from induced-dipole interactions and these are expected to produce a net repulsion between components in liquid mixtures. Most polyolefin blends are not compatible, while the miscible ones are driving research to provide reasons for their mixing. Possible factors influencing mixing, such as geometric effects, are being considered. For example, exploring if more favorable packing for one of the components in the blend versus self packing causes the molecules to prefer the packing offered by the blend. ${ }^{9}$ Work is also being done to elucidate how mixing seems to depend closely on the degree, the length, and the location of the sidegroup. ${ }^{1,12,14-17,27-30}$ Other factors such as polymeric microstructure, tacticity, comonomer incorporation, and chain dynamics, are being investigated for their contributing roles to the total enthalpy and entropy of the blend systems. 
Phase diagrams can be constructed for polymer blends, which show that two polymers have ranges of composition and temperature within which they are miscible. ${ }^{1,8,9}$ Most polymer blends display a lower critical solution temperature (LCST), meaning they will phase separate at higher temperatures over certain composition ranges. ${ }^{9}$ Broad ranges of polyolefin blends are being used in commercial applications, either on the boundaries of miscibility, or in the heterogeneous/multiphase state. The performance of the materials can be evaluated depending on its application, because in certain situations phase separation will cause failures. Therefore, research is being conducted to define miscibility windows and phase diagrams, which can guide the design of particular blends for specific applications.

\section{Methods of Studying Polyolefin Blends}

Polyolefin blends pose a challenge to scientists, in that typical techniques used to study blend miscibility are limited due to the chemical and physical similarities that exist between the components. ${ }^{1,9}$ The basic microstructure of polyolefins is $-\mathrm{CH}_{2-}$, with differences between individual polymers coming from the backbone and side groups like methyl $\left(\mathrm{CH}_{3}\right)$, methylene $\left(\mathrm{CH}_{2}\right)$, methine $(\mathrm{CH})$ and quaternary carbons $(\mathrm{C}){ }^{8}$ The challenge in studying polyolefin blends is rooted in their chemical and physical similarities, but the enhanced blend properties drive the research community to develop experimental techniques and mixing theories to resolve questions about miscibility and phase separation. ${ }^{8}$

Phase boundaries of polymer blends are often studied by comparing melt densities, refractive indecies, and glass transition temperatures, but these are often 
too similar in polyolefins, thereby precluding the utility of these methods. ${ }^{1}$ For example, a visual inspection of a blend is a quick measure of mixing, with miscible blends being clear, while immiscible blends displaying opaque or blurry features. Yet, most polyolefin blends studied to date are clear regardless of how intimately the component polymer chains are mixed.

Small-angle neutron scattering has provided the most useful and direct information regarding the thermodynamic interactions present in polyolefin blends. The single-phase, deuterated mixtures have been used to provide some insight into phase behavior as will be detailed later. Some successful studies have been conducted by observing cloud-points with cooling or heating, demonstrating that the light scattering increases due to phase separation. ${ }^{8}$ Thus, light scattering can be used to construct phase diagrams, which can be helpful for SANS data in pointing towards where phase boundaries are present and where to look for scattering. Since it does not require deuteration, light scattering has been helpful in determining the effects of deuteration on phase behavior. ${ }^{1}$ Glass transitions are also commonly measured using calorimetry or dynamic mechanical loss, and these measurements have shown two $\mathrm{T}_{\mathrm{g}}$ 's for two phase systems as opposed to one intermediate $\mathrm{T}_{\mathrm{g}}$ for a one phase system. ${ }^{8}$ But normally, these transitions are very broad and ineffective when comparing polyolefin blends with similar compositions. As a result, these measurements only provide preliminary information about a polyolefin blend system. Nuclear reaction analysis has been successfully used to determine amounts and compositions of phases, and can be combined with SANS to provide information on two-phase and one-phase blends 
using the same samples. ${ }^{1}$ A significant drawback to this method is the requirement of a nuclear reactor and deuteration of the polymers.

As seen above, deuteration has played an important role in exploiting methodology for polyolefin blend studies, yet it has been shown that it can have a significant effect on the data. The differences between protons and deuterons, quite small on the segmental level, have a significant effect when summed over an entire chain. ${ }^{31,32}$ The isotopic contribution can be detected most significantly near phase boundaries, especially the upper and lower critical points. Balsara et al. designed 50/50 blends such as D88/H78 and H88/D78. ${ }^{31}$ Here, the first letter specifies whether the polymer is fully hydrogenated or partially deuterated. The remainder of the naming scheme is as follows: for example, $\mathrm{H} 78$, denotes a hydrogenous polybutadiene or a statistical copolymer of ethylene and butene containing 78 weight percent of butene structure while D78 is the partially deuterated version. ${ }^{11}$ They found that by reversing the deuterium labels in the same blend, the upper critical temperatures differed by $70^{\circ} \mathrm{C} .{ }^{31}$ It was shown later that the thermodynamic interactions change differently depending on which component is deuterated, and that if neither component is labeled, the critical temperatures lie intermediate to the two blend ones. ${ }^{32}$ A correction scheme was devised to account for deuterium swapping, demonstrating that for a purely hydrogenous component the thermodynamic interactions can be estimated by taking the average of two blends with switched labels. ${ }^{32}$ Judging from the complexity of trying to make deuterated samples of both polymers to obtain the SANS data, plus disturbing the 'native' environment of the blend, the benefits of 
a noninvasive technique are clear. Solid-state NMR does not require or typically use isotopic labeling, but in certain cases is implemented at a low level to improve sensitivity. $^{13,18}$

\section{1-4 Current Theories for Polyolefin Blend Miscibility}

Theories based on solubility parameters, conformational asymmetry theory, and others such as the lattice cluster and polymer reactive interactive site model (PRISM) theory encompass some of the leading proposals for polyolefin miscibility. ${ }^{10}$ Due to their complexity, more in-depth descriptions will be given of two of the above: solubility parameters and conformational asymmetry theory. ${ }^{1}$

SANS (small-angle neutron scattering) is based on measurements on single-phase mixtures, where deuteration is a disadvantage due to the isotope effect. Graessley et al. used this method to determine $\chi$ values. ${ }^{11,12,14-16,27,28}$ They introduced the use of the interaction density coefficient or the interaction strength coefficient $\mathrm{X}$ as defined in Equation 1-3:

$$
X=\frac{\chi}{v} R T
$$

The denominator is a common reference volume, which allows for direct comparison between different blend systems and general conclusions to be established. Now, the Flory-Huggins equation becomes the following (Equation 1-4) with $v_{i} N_{i}=\mathrm{M}_{\mathrm{i}} / \rho_{\mathrm{i}}:^{1}$

$$
\Delta G_{m}=R T\left(\frac{\phi_{1} \rho_{1}}{M_{1}} \ln \phi_{1}+\frac{\phi_{2} \rho_{2}}{M_{2}} \ln \phi_{2}\right)+X \phi_{1} \phi_{2}
$$


The interaction strength can be expressed using solubility parameters if the polymers mix regularly so $\mathrm{X}$ is defined as indicated in Equation 1-5:

$$
X=\left(\delta_{1}-\delta_{2}\right)^{2}
$$

Solubility parameters of liquids are defined as $\delta=(\mathrm{U} / \mathrm{V})^{1 / 2}$ which is the cohesive energy density (CED). SANS determines the interaction strength using the random-phase approximation through the following equation, which is derived from Equation 1-4:

$$
\frac{1}{S(q)}=\frac{\rho_{1}}{M_{1} \phi_{1} P_{1}(q)}+\frac{\rho_{2}}{M_{2} \phi_{2} P_{2}(q)}-\frac{2 X}{R T}
$$

Where $\mathrm{S}(\mathrm{q})$ is the static structure factor for a single-phase blend (directly from SANS) and $\mathrm{P}_{\mathrm{i}}(\mathrm{q})$ is the form factor (independently obtained from SANS) determining the interaction strength $\mathrm{X}$.

Usually these measurements are correlated with PVT data of pure components to confirm the values from SANS or these values are used for irregular mixtures when the interaction strength is negative. ${ }^{11,12,15,27}$ Polymers are nonvolatile so cohesive energies cannot be measured. ${ }^{1}$ Internal pressure measurements have been found to be almost identical, so solubility parameters using PVT data can be assigned and compared with the SANS data directly by the following equation:

$$
\delta_{P V T}=\left(\frac{T \alpha}{\beta}\right)^{1 / 2}
$$


where $\alpha$ is the thermal expansion coefficient, and $\beta$ is the isothermal compressibility coefficient. Accordingly, $\chi=\left(\delta_{1}-\delta_{2}\right)^{2}$ sANS is almost identical with $\chi=\left(\delta_{1}-\delta_{2}\right)^{2}$ PVT for regular blends, which are around $75 \%$ of the blends made.

On the other hand, some polymers including PIB and PP (polypropylene) as one of the blend components mix irregularly by showing negative values of $\mathrm{X}^{11,12,14-16,27,28}$ This is an extremely surprising result that shows net attractions existing between these nonpolar chains. The way these blends are studied is by the degree of irregularity through direct comparison of the observed versus the expected values as determined with the following equation:

$$
X_{i r r}=X-\left(\delta_{1}-\delta_{2}\right)^{2}
$$

where $\mathrm{X}$ is the observed values from SANS and $\left(\delta_{1}-\delta_{2}\right)^{2}$ is the value from PVT. PIB was shown to have irregular mixing upon forming miscible blends by having large, negative $\chi$ values at room temperature for blends with: ${ }^{11}$

1. Ethylene-butene copolymers with $52-78 \% \mathrm{wt} \%$ butene

2. Alternating copolymer of ethylene and propylene with $60 \mathrm{wt} \%$ propylene

3. Alternating copolymer of ethylene and butene with $66 \mathrm{wt} \%$ butene

4. Head-to-head polypropylene

The authors showed that by increasing the temperature, the $\chi$ values increased rapidly and became positive as seen in Table 1-2. Some other conclusions about factors influencing miscibility drawn from these studies were the following: 
1. Blends miscible at room temperature phase separate at higher temperatures (40-190 $\left.{ }^{\circ} \mathrm{C}\right)$ showing LCST behavior.

2. Miscible blends with PIB have solubility parameters between $\delta_{50}-\delta_{80}$ (define the comonomer weight fractions).

3. Phase separation temperature is insensitive to component molecular weight.

4. Configurations such as head-to-head and head-to-tail have significant effects on miscibility.

5. Sequence order such as altering or statistical, only effects the temperature of phase separation but not the miscibility. ${ }^{11}$

Table 1-2 Interaction Parameters Determined by SANS (taken from Ref.11)

\begin{tabular}{|c|c|c|c|c|}
\hline & $\chi \times 10^{4}$ & $\chi \times 10^{4}$ & $\chi \times 10^{4}$ & $\chi \times 10^{4}$ \\
\hline blend & $27^{\circ} \mathrm{C}$ & $51^{\circ} \mathbf{C}$ & $83^{\circ} \mathrm{C}$ & $167^{\circ} \mathrm{C}$ \\
\hline PIBB / D52 $\left(\phi_{\text {PIB }}=0.49\right)$ & -18.2 & -2.5 & $2 \mathrm{P}$ & $2 \mathrm{P}$ \\
\hline PIBB / D66A $\left(\phi_{\mathrm{PIB}}=0.51\right)$ & -45.9 & -23.1 & -2.3 & $2 \mathrm{P}$ \\
\hline $\mathrm{PIBC} / \mathrm{D} 66 \mathrm{~A}\left(\phi_{\mathrm{PIB}}=0.49\right)$ & -43.7 & -21.5 & -1.0 & $2 \mathrm{P}$ \\
\hline PIBB / D78 $(\phi$ PIB $=0.46)$ & -21.4 & -8.7 & +3.8 & $2 \mathrm{P}$ \\
\hline PIBB / DhhPPA $\left(\phi_{\mathrm{PIB}}=0.47\right)$ & -101.0 & -72.0 & -37.4 & +10.1 \\
\hline PIBB / D52 $\left(\phi_{\mathrm{PIB}}=0.49\right)$ & -18.2 & -2.5 & $2 \mathrm{P}$ & $2 \mathrm{P}$ \\
\hline \multicolumn{5}{|l|}{$\begin{array}{l}\text { Note: } \\
\text { PIBB, } \mathbf{M}_{\mathbf{W}}=81.6 \times 10^{-3} \\
\text { PIBC, } \mathbf{M}_{\mathbf{W}}=160 \times 10^{-3}\end{array}$} \\
\hline \multicolumn{5}{|c|}{$\begin{array}{l}\text { D52 (model ethylene-butene copolymer with } 52 \% \text { butene } \rightarrow \mathrm{M}_{\mathrm{W}} 85 \times 10^{3} \\
\text { D66A (model ethylene-butene copolymer with } 66 \% \text { butene } \rightarrow \mathrm{M}_{\mathrm{W}} 114 \times 10^{3} \\
\text { D78 (model ethylene-butene copolymer with } 78 \% \text { butene } \rightarrow \mathrm{M}_{\mathrm{W}} 73 \times 10^{3} \\
\text { DhhPPA (model head-to-head polypropylene) } \rightarrow \mathrm{M}_{\mathrm{W}} 27.5 \times 10^{3}\end{array}$} \\
\hline
\end{tabular}

The authors did not have an explanation for the strong PIB attractions with the other blend components. They attributed the behavior to the anomalous properties of PIB and stabilization in those particular blends. ${ }^{11,12}$ 
In another work, regular mixing was seen for a miscible blend involving polypropylene (PP), which showed negative interaction parameter values similar to PIB. Consequently, solubility parameters could be assigned to each component and used to calculate $\mathrm{X}_{\text {irr. }}$ PP exhibited wide behavior with not only regular mixing, but also both attractive and repulsive irregularities, and the PIB-like net attractions. Two blends showing negative $\chi$ values with PP were the following:

1. Statistical copolymer of polyisoprene (50\% 1,4 and $50 \% 3,4$ content)

2. Statistical copolymer of ethylbutadiene ( $50 \% 1,4$ and $50 \% 3,4$ content $)^{12}$

For the PP blends, as opposed to the PIB blends, the temperature dependence of $\chi$ was much weaker meaning that $\chi$ values were smaller and did not change as rapidly with temperature. Overall, the SANS theory works for $75 \%$ of regular blends by showing a direct correlation between the solubility parameter and the packing length (which is the volume occupied by a polymer chain divided by the square of the radius of gyration). ${ }^{1}$

Bates and Fredrickson take advantage of the relationship between the solubility parameter and the packing length and apply it to a theory based on statistical segment length. ${ }^{10,17,29,30}$ They argue that contributions to entropy have only been considered on the local level and fail to account for the entropy that could exist on the non-local level upon mixing or 'long-ranged excess entropy'. According to this theory, which also uses SANS as an experimental method, conformational asymmetry exists in these polymer-polymer systems. Due to the limit of uniform polymer density, different components can possess various conformations. 
Their theory shows that a mismatch of component values will present a loss of configurational entropy upon mixing. Subsequently, a difference in conformational $\left(\mathrm{R}_{\mathrm{g}}\right)$ and volume-filling $(\mathrm{V})$ properties gives rise to excess entropy. These can be compared directly for a blend's components through the following parameter $\varepsilon$ :

$$
\begin{gathered}
\varepsilon=\left(\frac{\beta_{A}}{\beta_{B}}\right)^{2} \quad \text { where } \beta^{2}=\frac{R_{g}^{2}}{V} \\
R_{g}=L\left(\frac{N}{6}\right)^{1 / 2}
\end{gathered}
$$

Here, $\mathrm{L}$ is the statistical segment length and is a non-local parameter because its magnitude depends on excluded-volume interactions. ${ }^{17,}$ 29, $30 \mathrm{~A}$ comparison of two polyolefins, $\mathrm{PE}$ (polyethylene) and $\mathrm{PEE}($ poly(ethylethylene)) was done by Bates and Fredrickson showing minimal heat of mixing contributions, and negligible density differences. Due to similar molecular weights $\left(\mathrm{N}_{\mathrm{PE}}=\mathrm{N}_{\mathrm{PEE}}\right)$ and densities, their volumes are very similar $\left(\mathrm{V}_{\mathrm{PE}}=\mathrm{V}_{\mathrm{PEE}}\right)$, but the radius of gyrations will be different for both which will be reflected in the statistical segment length in Equation 1-10. From experimental data, $L_{P E}=8.2 \mathrm{~A}$ and $\mathrm{L}_{\mathrm{PEE}}=5.1$, where the polyethylene looks more like a thin chain while PEE resembles a much thicker and globular form. The non-local entropy described falls in between the length scale of $\left(\mathrm{L}_{\mathrm{PE}} \mathrm{L}_{\mathrm{PEE}}\right)^{1 / 2}$ and the radius of gyration length scale $\left(\mathrm{R}_{\mathrm{PE}} \mathrm{R}_{\mathrm{PEE}}\right)^{1 / 2}$. This non-local entropy can be introduced when local energy of mixing becomes negligible. 
Irregularity is strongly related with differences in local packing for polyolefin blends as seen by simulations done based on the radial pair-distribution function $\mathrm{g}(\mathrm{r}){ }^{1}$ Slightly positive values of $\mathrm{X}_{\text {irr }}$ indicate similarities in local order whereas negative values of $\mathrm{X}_{\text {irr }}$ point toward large deviations in local-order. Therefore, intramolecular energies describe the local conformations, which in turn give rise to local geometries that will pack in certain ways. For example, two homopolymers that are almost identical in structure will show small changes in local geometries upon blending. Consequently, irregularity will be zero or faintly positive. On the other hand, two homopolymers that are diverse in local structure will see an enhancement of geometries with blending. This could result in more efficient space filling and a negative $\mathrm{X}_{\mathrm{irr}}$ or less efficient packing and a positive $\mathrm{X}_{\text {irr }} .{ }^{1}$ More research needs to be done because at this time, the existing theories are unable to predict the outcome. Applying this theory to copolymers, which are diverse in their structure shows that blending will not change the packing efficiency because various conformations are already available. So with similar free-volume between the two copolymers, regular mixing should be seen. Predicting the properties of a mixture between a homopolymer and a copolymer is uncertain, and depends on the free-volume differences between the two and the packing differences obtained with blending. ${ }^{1}$

Other theories such as lattice cluster theory and polymer reactive interactive site model theory (PRISM) exist at this time but they will not be discussed further. As mentioned previously, local experimental data that could shed light on how polyolefins pack and at what level they interact with one 
another, is a key missing element. Our group has pursued magnetic resonance investigations of microscopic miscibility and dynamics in polyolefin blends using solid-state NMR methods. ${ }^{33-35}$

\section{Polyisobutylene (PIB)—Focus in our studies}

We are particularly interested in polyolefin blends that contain polyisobutylene as one component, not only due to our long-standing interest in this polymer, ${ }^{35,36}$ but also due to reports that it exhibits negative $\chi$ (negative enthalpy) behavior with certain other polyolefins as mentioned above. ${ }^{11}$ As discussed previously, there is no logical justification for the mixing because no chemical interactions exist between the chain segments. The publication demonstrating miscible PIB blends offers the possibility of a local packing contribution to the fortification of the attractions between the two components for that particular blend. As compared with other polyolefins, PIB has anomalies in properties like larger density $\left(0.915 \mathrm{~g} / \mathrm{cm}^{3}\right.$ at $23^{\circ} \mathrm{C}$ versus all others that range from 0.85 to $0.87 \mathrm{~g} / \mathrm{cm}^{3}$ ), and much smaller thermal expansion $(\alpha)$ coefficient and isothermal compressibility $(\beta)$. The PIB molecules seem to be tightly arranged in the liquid state with their intermolecular forces being able to resist more strongly the perturbing effects of pressure and temperature. ${ }^{11}$ The authors speculate that the existing interactions are very unique for PIB in these blends, and they are responsible for the strong stabilization of the single phase blends.

Our group has shown that PIB exhibits chain-level mixing with head-tohead polypropylene (hhPP), ${ }^{33}$ while others have reported that it phase separates in blends with normal atactic head-to-tail PP. ${ }^{11}$ Given the unique chain-packing 
properties of PIB (which give rise to its barrier properties), we feel that this apparent regio-selectivity might arise from differential interchain packing geometries. Based on previous reports by Graessley, Lohse, and coworkers, ${ }^{12,}{ }^{14-}$ $16,27,28$ we are studying blends of PIB with poly(ethylene-co-1-butene) copolymers (PEB), in which the relative PEB comonomer concentration is either 23 or $66 \mathrm{wt} \%$ (13 and 49 mole\%, respectively). ${ }^{13}$ Our goals in this work are to interrogate length scales of mixing in the absence of extensive deuterium labeling, as well as determine the relationship between local backbone conformational barriers and miscibility.

\section{1-5 Solid-State Nuclear Magnetic Resonance}

Spin angular momentum (I) is a result of the ratio of protons and neutrons in an atomic nucleus, and is the reason why nuclear magnetic resonance is possible. The Newtonian model describes how the spinning motion, creates a magnetic moment, which produces a small magnetic field as seen with the following relationship:

$$
\mu=\gamma I
$$

where $\mu$ is the magnetic moment and $\gamma$ is the gyromagnetic ratio. (The gyromagnetic ratio is characteristic for each nucleus, for example proton's $\gamma$ is $2.6752 \times 10^{7} \mathrm{rad} \mathrm{T}^{-1} \mathrm{~s}^{-1}$ ). Thus, when a magnetic field $\mathrm{B}_{0}$ is applied, the magnetic moments precess with an angular frequency $\omega_{0}$ about the z-axis, whose magnitude is the Larmor frequency as seen in Equation 1-12: 


$$
\omega_{o}=\gamma B_{o}
$$

Interactions with $\mathrm{B}_{0}$ lead to various energy spin states, with a slightly larger population in the lower energy state. Application of a radiation frequency exactly matching the energy gap induces transitions between states. For instance, a $90^{\circ}$ pulse will create phase coherences in the xy-plane and a signal that will decay with a certain time constant. The signal is influenced by many possible timedependent and time-independent interactions, which are summarized by the following Hamiltonian:

$$
H=H_{z}+H_{R F}+H_{D}+H_{C S}+H_{Q}+H_{I}
$$

Here, the Zeeman interaction is the largest but does not contain structural information; the last four terms are the dipole-dipole, chemical shift, quadrupolar, and scalar coupling interactions. The power of NMR lies in manipulating the radio frequency pulses in such a way as to gain information about the molecular structure and dynamics.

Solid-state NMR has, in many instances, evolved into a routine technique for studying structure and dynamics in a variety of solid systems with high resolution and sensitivity. The development of various techniques to probe internuclear distances, molecular dynamics, spin diffusion, and anisotropy has occurred by taking advantage of the various interactions between nuclear spins and the magnetic fields. ${ }^{37}$ In polymers, the focus has commonly been on observing spin $1 / 2$ nuclei, such as ${ }^{13} \mathrm{C}$ or ${ }^{15} \mathrm{~N}$ nucleus. An assortment of spin interactions are depicted in a solid spectrum, which in turn have to be manipulated 
in order to achieve high resolution. The three principal interactions responsible for the broad lines detected in a solid spectrum are heteronuclear dipolar coupling, homonuclear dipolar coupling, and chemical-shift anisotropy. These interactions also exist in a solution spectrum, but due to the fast tumbling of the molecules, are successfully averaged to zero and eliminated.

\section{Heteronuclear Dipolar Coupling}

The origin of the heteronuclear dipolar coupling is from the interaction between nuclear magnetic moments of two different nuclear spins (their two magnetic fields), assigned as I for abundant nuclear spins, and $\mathrm{S}$ for rare ones. ${ }^{37}$ The Zeeman interactions, $E_{\text {Zeeman }}$, illustrates the energy of spin I and S, depending on whether they are aligned parallel or antiparallel with the external field:

$$
E_{\text {Zeeman }}=-\left(\frac{h}{2 \pi}\right) \gamma B_{o} m_{I}
$$

Equation 1-14 includes $\gamma$ (gyromagnetic ratio), $\mathrm{B}_{0}$ (external field) and $\mathrm{m}_{\mathrm{I}}$ (nuclear spin quantum number being either $+1 / 2$ or $-1 / 2$ for spin $1 / 2$ nucleus). The internuclear distance between $\mathrm{I}$ and $\mathrm{S}$ determines how much the resonance frequency of spin S can be altered by spin I (which will align either with or against the field), resulting in an increase or decrease of the local magnetic field at spin S. The potential power of spin I on the magnetic field of S, or the possible impact of the heteronuclear dipolar coupling, is described by the following Hamiltonian:

$$
H_{I S}=-D\left(3 \cos ^{2} \theta-1\right) I_{Z} S_{Z}
$$


The dependence of the interaction between I and $\mathrm{S}$ and the main field $\left(\mathrm{B}_{0}\right)$ is represented by the angle $\theta$, made by the vector between $\mathrm{I}$ and $\mathrm{S}$ and the direction of $\mathrm{B}_{0}$, while $d$ represents the dipolar coupling constant which corresponds to:

$$
D=\left(\frac{\mu_{o}}{4 \pi}\right) \frac{h \gamma_{I} \gamma_{S}}{2 \pi r_{I S}{ }^{3}}
$$

Here, $\mu_{0}$ is the permeability of free space, $r_{I S}$ is the internuclear distance, $\gamma_{I}$ and $\gamma_{S}$ are the gyromagnetic ratios of the I and S spins, and finally, $I_{Z}$ and $S_{Z}$ are the $z$ components of the nuclear spin angular momentum operators $\mathrm{I}$ and $\mathrm{S}$. Heteronuclear dipolar coupling is a through-space interaction, and as a result, diminishes the greater the distance between the two nuclei, as well as being orientation dependent, meaning that for certain orientations, the dipolar coupling can be greater. In order to eliminate the spectral broadening, characterized by coupling constants as great as $30 \mathrm{kHz}$, proton spins are manipulated by coherent radio frequency field such that they are time-averaged out. Steady radiofrequency pulses at the ${ }^{1} \mathrm{H}$ Larmor frequency rotate the proton nuclear spins from their parallel (up) and antiparallel (down) states, resulting in the heteronuclear coupling being averaged away. The method of continuous wave (CW) spin decoupling is more effective in narrowing the signal the closer the ${ }^{1} \mathrm{H}$ and ${ }^{13} \mathrm{C}$ nuclei are to each other. ${ }^{37,38}$

\section{Homonuclear Dipolar Coupling}

Homonuclear dipolar coupling is very similar to the heteronuclear dipolar coupling discussed above, but occurs for like spins. The major difference is that 
heteronuclear coupling is between two unlike spins (two different resonant frequencies), while homonuclear coupling occurs between two like spins (same resonance frequency). As a consequence, energy-conserving spin flips (spindiffusion) can occur for the homonuclear case, where magnetization is exchanged with one spin flipping up while the other spin flips down as seen in Figure 1-4.

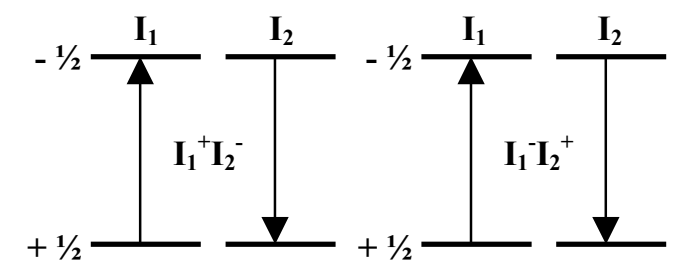

Figure 1-4 Schematic representing exchange of magnetization via energy conserving flip-flops (taken from Ref. 37).

Due to the different nuclear transition frequencies in the heteronuclear case, this mechanism can not take place. Therefore, the Hamiltonian also differs slightly between the two couplings as demonstrated in Equation 1-17:

$$
H_{I I}=-1 / 2 D\left(3 \cos ^{2} \theta-1\right)\left(3 I_{1 Z} I_{2 Z}-\left(I_{1} \bullet I_{2}\right)\right)
$$

where $\left(I_{1} \bullet I_{2}\right)$ is equal to $I_{1 X} I_{2 X}+I_{1 Y} I_{2 Y}+I_{1 Z} I_{2 Z}$. Rewriting this equation in terms of raising and lowering operators $\left(\mathrm{I}^{+}=\mathrm{I}_{\mathrm{x}}+\mathrm{iI}_{\mathrm{y}}\right.$ and $\left.\mathrm{I}^{-}=\mathrm{I}_{\mathrm{x}}-i \mathrm{I}_{\mathrm{y}}\right)$, the following Equation 1-18 is derived:

$$
H_{I I}=-\frac{D}{2}\left(3 \cos ^{2} \theta-1\right)\left(2 I_{1 Z} I_{2 Z}-\left(\frac{I_{1}^{+} I_{2}^{-}+I_{1}^{-} I_{2}^{+}}{2}\right)\right)
$$


The terms accounting for the flip-flop mechanism $\left(\mathrm{I}_{1}{ }^{+} \mathrm{I}_{2}{ }^{-}+\mathrm{I}_{1}{ }^{-} \mathrm{I}_{2}{ }^{+}\right)$correspond to raising an operator $\mathrm{I}^{+}$, where the spin is rotated from down to up position at the same time as the lowering of an operator $\mathrm{I}^{-}$, flips the spin from up to down position. Due to the great quantity of protons in polymer systems and their strong coupling between one another (up to $100 \mathrm{kHz}$ because of their large gyromagnetic ratios), decoupling is hard to accomplish, requiring either coherent multiple radio frequency pulse trains, or extremely fast MAS. ${ }^{37,38}$

\section{Chemical Shift Anisotropy}

The distribution of electrons shielding the nucleus from the main field around the molecule of interest determines the combined affect that they will have on the resonance frequency at the given nuclear spin. ${ }^{38}$ With the placement of the nuclei in the main field, the encircling electrons begin to create local magnetic fields via circulating currents resulting in small additions or subtractions to the nuclei's magnetic field. Typically, the electron distributions in a molecule are not symmetrical, causing various environments for particular positions in the magnetic field and thus a distribution of chemical shifts. ${ }^{37,38}$

The molecule's orientation in $\mathrm{B}_{0}$ will establish the perturbation extent of the electron density on the resonance frequency; when the widest part of the electron cloud is oriented along $\mathrm{B}_{0}$, a smaller chemical shift will occur (shielding) whereas when the narrowest part is positioned along $\mathrm{B}_{0}$, a large shift will take place (deshielding). In a sample where all possible orientations are present, a broad CSA pattern will occur, with the left and right edges representing the $\delta_{11}$ and $\delta_{33}$ chemical shifts, while the maximum intensity would be $\delta_{22}$. For a case 
where a molecule is axially symmetric, $\delta_{11}=\delta_{22}$, the chemical shift Hamiltonian is the following:

$$
H_{C S}=\delta B_{o} I_{Z}\left(\delta_{\text {iso }}+\frac{\delta_{C S A}}{2}\left(3 \cos ^{2} \theta-1\right)\right)
$$

Chemical shift possesses an isotropic and an anisotropic contribution which are defined as the following:

$$
\delta_{\text {iso }}=\frac{1}{3}\left(\delta_{11}+\delta_{22}+\delta_{33}\right) \quad ; \quad \delta_{C S A}=\delta_{33}-\delta_{\text {iso }}
$$

Therefore, with rapid tumbling in solution or spinning at an angle of $54.74^{\circ}$ in a solid, only the isotropic component will remain and the broadening due to CSA is removed. Yet, part of the motivation behind pursing solid-state NMR was to observe these CSA patterns that contain vital structural information. It is much easier to observe changes in molecular structure by observing changes in the individual principal values, rather than looking towards a change in $\delta_{\text {iso }}$, which only changes very slightly. ${ }^{37,38}$

\section{1-6 Objectives}

The specific purpose for the research presented in the following chapters was to demonstrate the potential of SSNMR as an essential tool in studying polyolefins and their blends. A comprehensive review of metallocene catalysis, polyolefin blends and polymer mixing is given in Chapter 1. Several experimental approaches for characterizing polyolefin blends, many utilizing variable 
temperature capabilities, are described in Chapter 2 with special emphasis on exact pulse sequences. Chapter 3 includes a publication on a crystalline solid, which came about as a result of the original, 2D-Exchange experiment calibration. A comprehensive study of two polyolefin blends via the fully developed SSNMR techniques is presented in Chapter 4. Polyolefins are used as model systems in Chapter 5 and 6 towards addressing fundamental polymer science questions, focusing mainly on length scales in amorphous polymers, timescale of the glass transition, and configurational entropy in polymers. Lastly, CODEX, a recently developed alternative to 2D exchange, reveals dynamics data on a new polyolefin system in Chapter 7. 
CHAPTER 2: Experimental Literature Review 


\section{2-1 Material Characterization}

\section{Types and Compositions of Blends Studied}

Polyolefins comprise an economically and technologically vital field that with continuing improvements in metallocene polymerization catalysis, have showed enhanced physical properties accessible through copolymerization and blending. Due to the increased available compositions of the ethylene- $\alpha$-olefin copolymers, the goals are to design miscibility windows in blends of polypropylene, polyisobutylene, or ethylene- $\alpha$-olefin copolymers. The numerous

Table 2-1 Monomer structures of polyolefins studied

\begin{tabular}{|c|c|}
\hline$-\left(-\mathrm{CH}_{2}-\left.\right|_{\mathrm{C}} ^{\mathrm{C} \mathrm{H}_{3}}\right.$ & $-\left(\mathrm{CH}_{2}-\stackrel{\mathrm{CH}_{3}}{\mathrm{CH}}-\right.$ \\
\hline 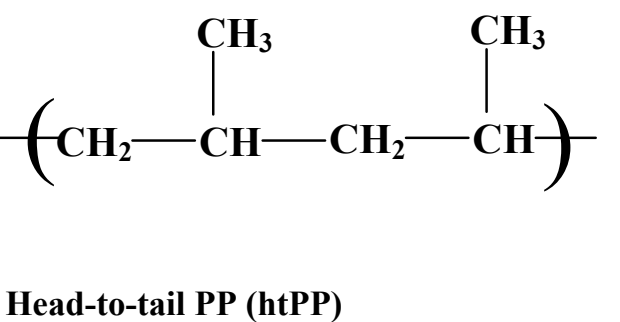 & $\begin{array}{l}\left(-\mathrm{CH}_{2}-\stackrel{\mathrm{CH}}{\mathrm{C}}-\stackrel{\mathrm{H}_{3}}{\mathrm{C}} \mathrm{CH}-\mathrm{CH}_{2}\right)- \\
\text { Head-to-head PP (hhPP) }\end{array}$ \\
\hline 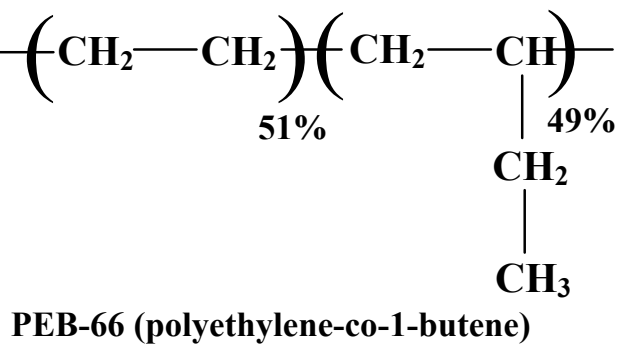 & 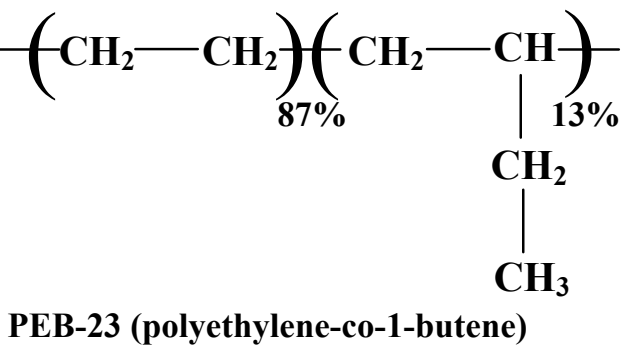 \\
\hline
\end{tabular}


50/50 weight percent blends is straight-forward with dissolution in toluene for 2448 hours, followed by solvent evaporation, and then vacuum-drying to $10^{-2}$ Torr for 4 days or longer. Typically, all blends, whether subsequently concluded to be miscible or immiscible, looked clear upon drying.

\section{Solution and Solid State NMR for the Characterization of Polyolefins}

A polymer's physical properties are the result of its specific microstructure. A polymer can be described with the type of side chains present, stereosequence, regiosequence, and presence/absence of branching. ${ }^{40,}{ }^{41}$ Solution

${ }^{13} \mathrm{C}$ NMR has proven vital as a tool in determining polymer stereosequences. ${ }^{40}$ The extreme sensitivity of ${ }^{13} \mathrm{C}$ solution NMR to a polymer's microstructure is shown in Figure 2-1, where three different polypropylene samples produce three diverse spectra due to each sample having specific stereoregularities. Upon comparing the three spectra carefully, it can be concluded that the atactic sample has overlapping peaks from the isotactic and the syndiotactic polymers in addition to other extra resonances. A number of studies showed that the multiplicity in each carbon's resonance was not only due to the $\alpha$-, $\beta$-, $\gamma$-substituent effects, but also due to stereosequence-dependence on the local conformations over as many as six backbone bonds for the polypropylene sample. ${ }^{40}$ It was concluded that a $\gamma$ substituent in a gauche arrangement can be as powerful as $\alpha$ - or $\beta$-substituents on the chemical shift (around -5-7 ppm vs. +9 ppm) as well being sensitive to the local conformation. ${ }^{41}$ The $\gamma$-substituent has to be in the gauche conformation in order to be effective, and it has proven to be the primary tool in assignment of ${ }^{13} \mathrm{C}$ spectra. 
In the solid state, the molecular rigidity prevents averaging and much broader resonances are observed. A great deal of conformational data can be revealed by a solid ${ }^{13} \mathrm{C}$ NMR spectrum, as observed in Figure 2-2 (a), where atactic polypropylene shows various conformations present via the methylene and the

(a)

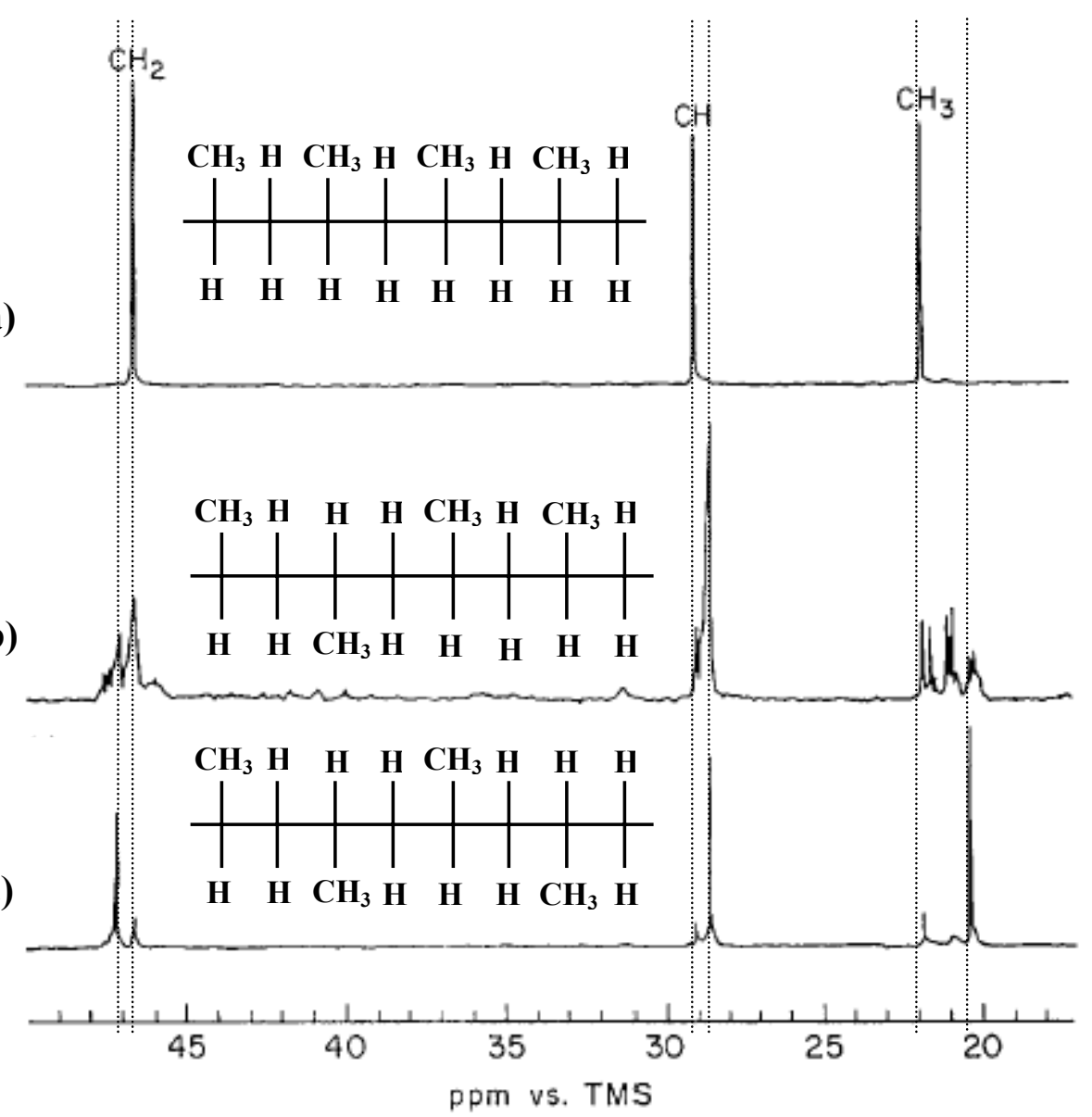

Figure 2-1 Several ${ }^{13} \mathrm{C}$ NMR spectra run at $25 \mathrm{MHz}$ of (a) isotactic, (b) atactic, and (c) syndiotactic PP. (The solvent used was 1,2,4-trichlorobenzene at $140^{\circ} \mathrm{C}$ ) (taken from Ref. 40).

methyl peaks. The preferred repeating conformations of iPP and sPP can be combined together with the $\gamma$-gauche effect, and the three most prevalent conformations for the methylene region assigned as $t t$ (trans-trans) from $\mathrm{sPP}, t g / g t$ 
(trans-gauche and gauche-trans) from iPP and $g g$ (gauche-gauche) from sPP. ${ }^{41,42}$ The $\mathrm{CH}$ and $\mathrm{CH}_{3}$ groups both have similar conformational populations as seen in iPP and sPP with an extra peak at $18.2 \mathrm{ppm}$ caused by the specific conformational effects in aPP. The relative geometric conformations for a tetrameric unit of the amorphous polypropylene are pointed out in Figure 2-3, demonstrating via Newman projections how the $\gamma$-gauche effect produces the relative distributions of the three different conformational populations.

(a)

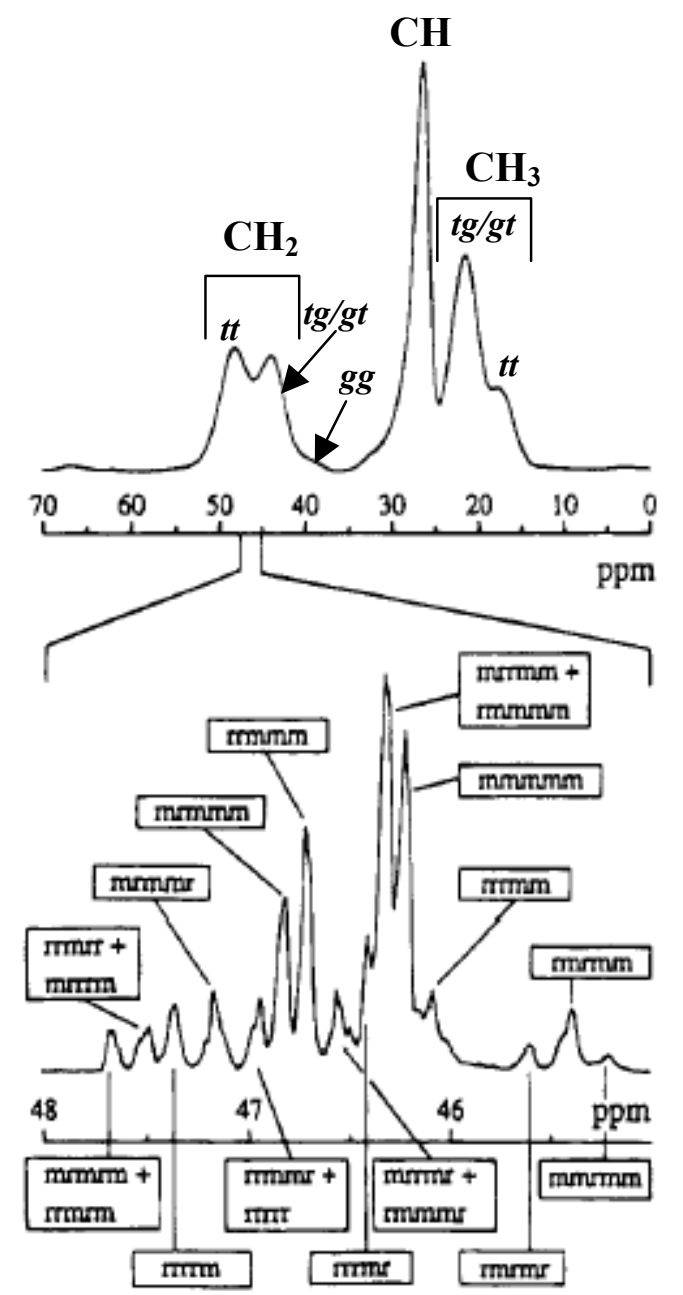

Figure 2-2 (a) Top spectrum is a solid-state ${ }^{13} \mathrm{C}$ CPMAS spectrum of aPP demonstrating the broad resonances of the methylene and methyl groups. Much narrower solution resonances can be seen for just the methylene region in (b) representing particular stereosequences (taken from Ref. 42). 
As demonstrated by Figure 2-2, the combination of solution and solidstate NMR can be a powerful weapon in investigating the configurations and conformations present within the polymer chain. Due to fast motion and thus fast trans-gauche conformational transitions present in solution NMR, the displayed chemical shifts reflect conformationally-averaged environments. Thus each configuration will have a different average conformation, resulting in a distinct isotropic chemical shift and much narrower lines then in a solid spectrum. ${ }^{41,42}$

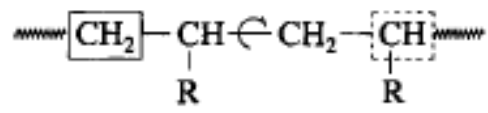

(a)
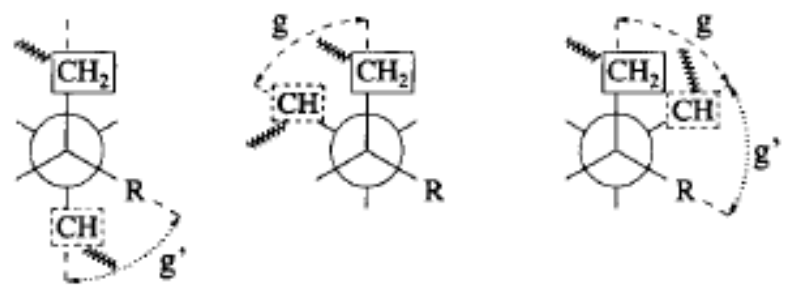

(b)
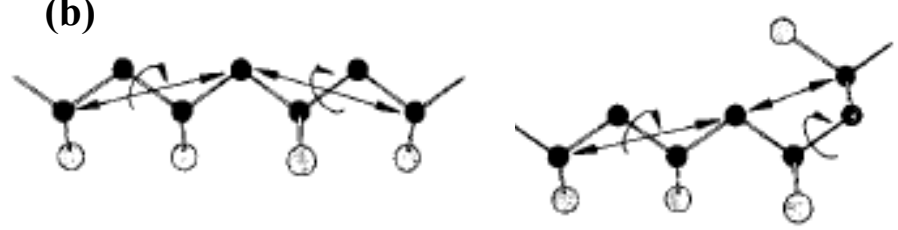

trans / gauche ( $\left(\mathrm{t}^{*} . * \mathrm{~g}\right)$

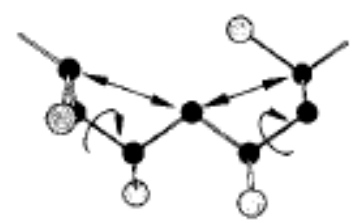

$\operatorname{trans} / \operatorname{trans}\left(t^{* * *} \cdot \mathbf{t}\right)$

gauche / gauche $\left(g^{*} \cdot * g\right)$

Figure 2-3 Schematic representations of the three different conformations present for the methylene regions in atactic polypropylene. (a) Newman projections of the conformations of a single PP configuration (mrr). As seen in the figure, $\mathrm{g}$ (gauche) conformation marks an interaction involving $\mathrm{CH}_{2}$, while g'(gauche) involves the main-chain $\mathrm{CH}$ and $\mathrm{R}$. (b) A chain schematic showing the same configuration, and the conformations responsible for the three populations in the methylene region as effected by the $\gamma$-gauche effect (taken from Ref. 42).

\section{Glass Transition in Amorphous Polymer Blends}

Amorphous polymers experience a glass transition $\left(\mathrm{T}_{\mathrm{g}}\right)$, which is the change between rubbery liquid and the glassy state or in other words, detection of 
the onset of molecular motion in the form of interconversion of backbone conformations. ${ }^{43}$ It is usually measured by differential scanning calorimetry (DSC) where the changes in specific heat of the polymer are measured. The $T_{g}$ depends on the rate at which heat flow is supplied for example; if the sample is heated quickly, the $T_{g}$ will occur at a higher temperature then if it were heated more slowly. The change from glass to rubber is not abrupt, occurring over temperature ranges up to $10^{\circ} \mathrm{C}$. DSC can be used to establish the miscibility of a blend, by observing a single, intermediate $\mathrm{T}_{\mathrm{g}}$ for a miscible blend as opposed to two $\mathrm{T}_{\mathrm{g}}$ 's for an immiscible blend. Due to the fact that the glass transition is rate dependent, different methods can yield different results. If the technique has long observation times, it will be sensitive to slow motions, whereas if it has short observation times, only fast motion will be detected. ${ }^{43}$

\section{2-2 Temperature calibration of the Bruker MAS Probe}

Lead nitrate has become a vital tool in MAS probes to accurately detect temperature changes and gradients over a wide temperature range. ${ }^{44}$ The $4-\mathrm{mm}$ Bruker MAS probe temperature was measured using the $\mathrm{Pb}\left(\mathrm{NO}_{3}\right)_{2}$ chemical shift thermometry method due to the high sensitivity, sharp NMR lines, and

temperature dependent chemical shifts. ${ }^{207} \mathrm{~Pb}$ has linear temperature dependence over a range of temperatures $\left(-100^{\circ} \mathrm{C}\right.$ to $\left.+150^{\circ} \mathrm{C}\right)$ presumed to be due to thermal lattice expansion. ${ }^{44}$ Typically, the variable temperature electronics are first calibrated to allow for a systematic error arising from the thermocouple not being 
in intimate contact with the sample. The correction is acquired by observing melting points and the temperature readings corrected accordingly. ${ }^{44}$

As seen in Figure 2-4, the analysis of the data is usually simplified by restricting the sample to two regions in the rotor, bottom end and middle, separated by inert-teflon plugs. By using the two restricted regions, one can clearly observe the temperature gradient inside the rotor by the shift difference between the large peak from the center sample and smaller peak from the bottom portion. If a completely filled rotor is used, the resonance will be a single broad line with the shape determined by the temperature gradients present.

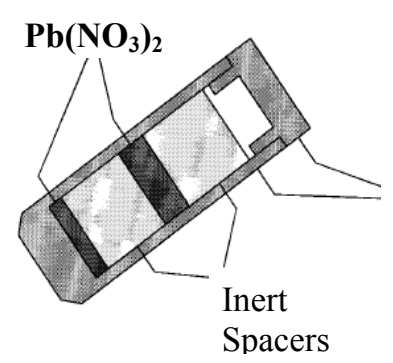

(a)

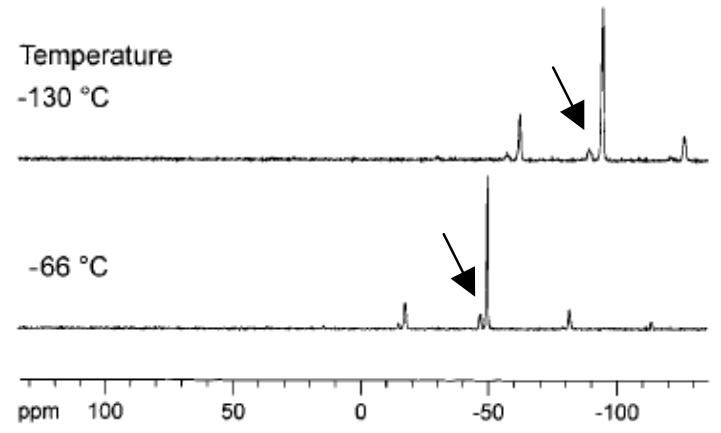

(b)

Figure 2-4 (a) Lead nitrate test sample where the center-sample provides the primary temperature measurement, and the bottom part measures the axial temperature gradient as demonstrated in (b) with a large centerband and a small peak from the bottom sample portion (taken from Ref. 44).

A series of experiments ranging from $203 \mathrm{~K}\left(-70^{\circ} \mathrm{C}\right)$ to $350 \mathrm{~K}\left(77^{\circ} \mathrm{C}\right)$ were run in steps of $10-20 \mathrm{~K}$ on a sample with lead nitrate restricted to the middle of the rotor. We were concerned with the ability to reproduce identical temperatures and did not need to consider the rotor gradients. At each temperature, around 20 minutes were allowed for sample equilibration, following which each spectrum was collected at a spinning rate of $2000 \pm 2 \mathrm{~Hz}$ with a $3.5 \mu$ s $90^{\circ}$ pulse, relaxation 
delay of 10s, and eight scans. Each calibration was done with 2000 L/min cooling gas flow rates to minimize temperature gradients in the sample. Figure 2-5 shows the sensitivity of using $\mathrm{Pb}\left(\mathrm{NO}_{3}\right)_{2}$ as indicated by the strong signal and a strong temperature dependence of the shift relative to the linewidth. Spinning sidebands are also observed, which vary with temperature indicative of a change in the
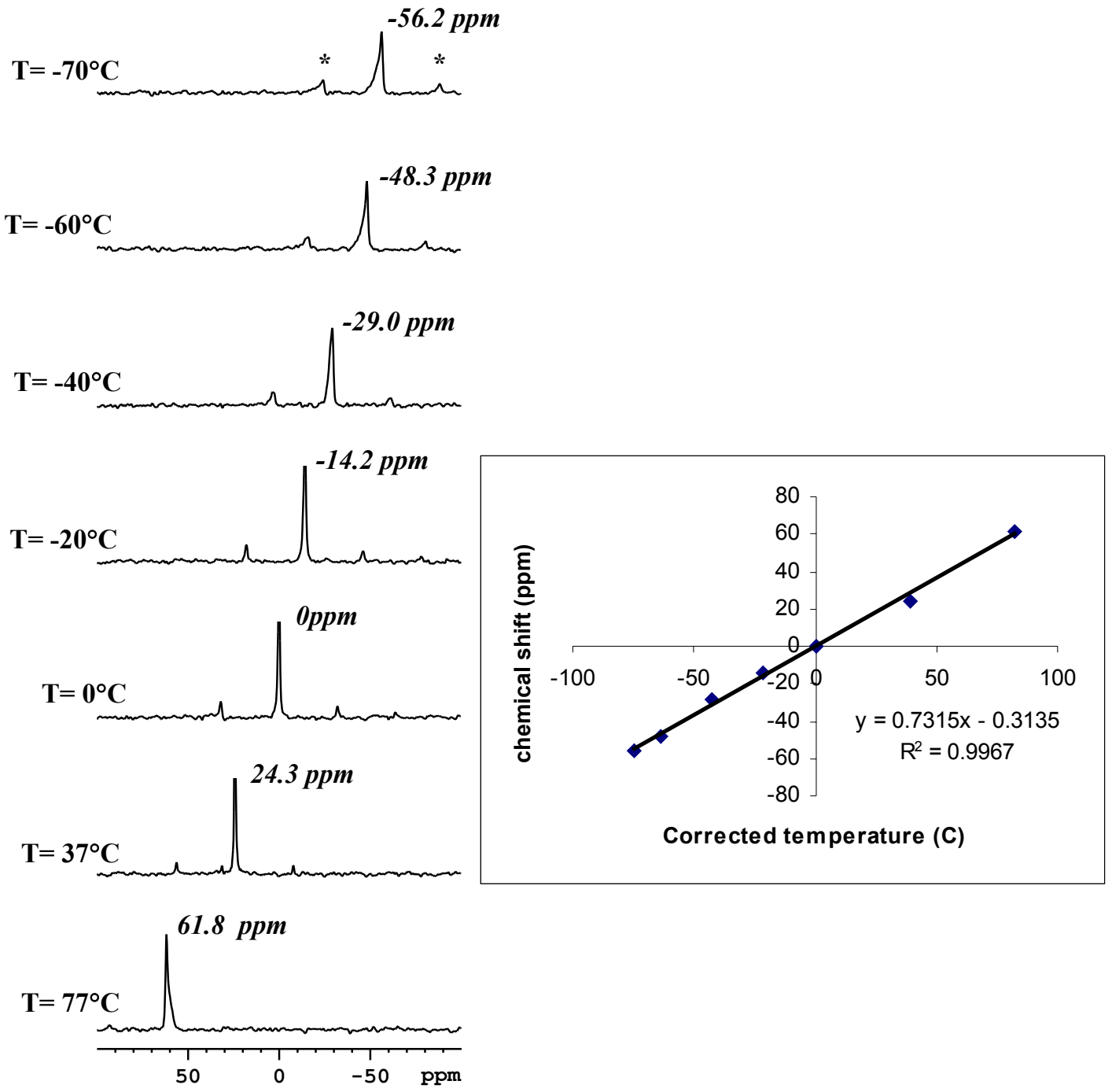

(a)

(b)

Figure 2-5 (a) ${ }^{207} \mathrm{~Pb}$ MAS spectra as a function of temperature. Spinning sidebands appear at intervals of $2000 \mathrm{~Hz}$ (indicated by ${ }^{*}$ ) and $0 \mathrm{ppm}$ corresponds to $0^{\circ} \mathrm{C}$. (b) Graph showing the expected linear dependence of the chemical shift with temperature. 
CSA. When a plot of the shift relative to the temperature is constructed with the chemical shift scale adjusted so $0 \mathrm{ppm}$ is $0^{\circ} \mathrm{C}$, a linear dependence is indeed observed. Due to the single resonance of $\mathrm{Pb}\left(\mathrm{NO}_{3}\right)_{2}$ (no built-in shift reference), a single spectrum cannot give an absolute temperature measurement. When a spectrum is taken at room temperature, where the probe temperature gradients are small and the thermocouple reading is the closest to true sample temperature, an accurate reference can be established. By repeating the same experiment within a two-week time span and collecting almost identical spectra, we confirmed that the variable temperature conditions were stable and accurate.

\section{2-3 Relaxation and Dipolar Filter Experiments}

Spin relaxation times are vital in obtaining high-resolution solid-state NMR spectra at all temperatures. The basic $T_{1}$ and $T_{2}$ govern the $z$ and $x, y$, components return to equilibrium, so for example, the cross-polarization method has been useful in overcoming issues of long $\mathrm{T}_{1 \mathrm{C}}$ values, by basing the experimental repetition rates on the shorter proton values. Resolution advancements in solid-state carbon NMR have allowed for relaxation data to be acquired for distinct nuclei, as a consequence leading to elucidation of rigid body and local motions. ${ }^{38}$ The direct relationship between molecular motion and relaxation enables experimental relaxation rates to reveal correlation times of the molecular motions. Relaxation parameters depend on both the isotope and its individual environment as determined by various Larmor frequencies, motions and the accessible relaxation mechanisms. Numerous types of relaxation 
parameters exist, but in polymers the main focus lies in characterizing the nature of molecular motions in the $\mathrm{MHz}$ (Larmor frequencies) and $\mathrm{kHz}$ frequency ranges by variable temperature spin lattice relaxation $\left(T_{1}\right)$ as well as spin lattice relaxation in the rotating frame $\left(\mathrm{T}_{1 \rho}\right)$, respectively. ${ }^{45},{ }^{46}$ Schaefer and Stejskal described early experimental developments on glassy polymers by using $T_{1 \rho}$ measurements to explore dynamic heterogeneities. ${ }^{47}$

Proton $T_{1 \rho}$ measurements have provided evidence for compatibility in solid polymer blends proving to be a dominant method in studying local polymer structures together with following molecular dynamics with blending. ${ }^{35,38,47}$ Due to the strong dipolar coupling and efficient spin-diffusion (energy-conserving spin

Table 2-3 Selective $\mathrm{T}_{1 \rho}{ }^{\mathrm{H}}$ values on two different systems (taken from Ref. 47).

\begin{tabular}{|l|c|}
\hline Polymer & $\mathbf{T}_{\mathbf{1} \boldsymbol{p}}{ }^{\mathbf{H}}$ Values (ms) \\
\hline $\mathrm{PPO}$ & 49 \\
\hline$\underline{\mathrm{PPO}} / \mathrm{PS}$ & 6.7 \\
\hline $\mathrm{PPO} / \underline{\mathrm{PS}}$ & 6.2 \\
\hline & 5.6 \\
\hline $\mathrm{PPO}$ & 49 \\
\hline$\underline{\mathrm{PPO}} / p$-Cl-PS & 48 \\
\hline $\mathrm{PPO} / p$-Cl-PS & 9 \\
\hline$p$-Cl-PS & 8 \\
\hline
\end{tabular}

flips) that exists in solid systems, the $\mathrm{T}_{1 \rho}{ }^{\mathrm{H}}$ values are averaged over the regions of protons present. For two polymers, which were blended, the proximity of two different polymer chain will determine the rate of spin-diffusion amongst the protons. Typically, for an intimately mixed set of chains, fast spin diffusion gives single $\mathrm{T}_{1 \rho}{ }^{\mathrm{H}}$ values, while an immiscible one offers dissimilar values as seen in Table 2-3. The PPO/PS blend (with composition of 25/75) had intermediate 
values as compared with the individual numbers for the PPO and PS indicating a compatible blend. As demonstrated by Stejskal et al., by acquiring proton $\mathrm{T}_{1 \rho}{ }^{\mathrm{H}}$ values via carbons, they were able to show slight differences in the individual $\mathrm{T}_{1 p}{ }^{\mathrm{H}}$ values, explaining the slight difference in the blend values as small PS regions mixed on a different scale than the rest of the blend. ${ }^{47}$ On the other hand, the quenched $\mathrm{PPO} / p$-Cl-PS (75/25 composition) blend had basically the same $\mathrm{T}_{1 \rho}{ }^{\mathrm{H}}$ values for the blend as it showed for individual components indicating no spin diffusion between the two polymers, and as a result, very little interchain mixing. The key in using $\mathrm{T}_{1 \rho}{ }^{\mathrm{H}}$ measurements to prove intimate mixing is the ability to resolve distinct resonances from each of the components in the blend thus allowing the changes from the homopolymer to the blend values to be followed. An important restriction when using this approximation is that the values of the relaxation time constants for the two homopolymers should differ by a factor of 2-3 thus the existence or the absence of convergence for the blend can be identified. As mentioned previously, molecular dynamics can be followed where the $\mathrm{T}_{1 \rho}{ }^{\mathrm{H}}$ will get smaller with increasing polymer mobility, while with growing rigidity (e.g. at temperatures below $\mathrm{T}_{\mathrm{g}}$ ), the values will enlarge. ${ }^{45,46}$ Once motion sets in, and is adequately fast to average out the dipolar interactions (in polymers often being $\mathrm{C}-\mathrm{H}$ and $\mathrm{H}-\mathrm{H}$ interactions), spin diffusion is prevented from taking place. Now, the $\mathrm{T}_{1 \rho}{ }^{\mathrm{H}}$ measurement monitors the local motions taking place.

\section{Variable Temperature Studies}

Variable temperature relaxation data may be used to monitor the change in 
the molecular motions distribution, while later following the level of molecular scale chain mixing. As discussed previously, $\mathrm{T}_{1 \rho \mathrm{H}}$ data monitors the level of mixing at the chain level, and as an example, this was demonstrated for a blend of aPP with PEE (poly(ethylene)ethylethylene). ${ }^{35}$ Here, solid-state NMR is advantageous in that the effect of blending on chain dynamics can be followed independently for each component due to separate resonances, with the $\mathrm{CH}_{2}$ signal being monitored for aPP, while the $\mathrm{CH}_{2}$ and $\mathrm{CH}_{3}$ resonances are observed for the PEE. As seen in Figure 2-6, the relaxation times over the wide temperature range for the blend components fall in between the homopolymers, yet they do not converge to the same value. The convergence is expected if the two polymers are mixed intimately due to spin-diffusion being active. Consequently, it was concluded that the chains were mixed, but in certain parts of the blend were phase separated, exceeding the spin-diffusion length scale.

On the other hand, the authors also present an example of a blend not mixed at the chain level, as observed by the almost identical $\mathrm{T}_{1 \rho \mathrm{H}}$ data for the blend (aPP/PE-DD where PE-D is poly(ethylene-co-dodecene)) and the homopolymer, aPP. Here, the blending has no effect on aPP, and the two polymer chains are not close enough for any spin-diffusion to take place. In the same graph, yet another blend's, aPP/PE-OD (where PE-OD is poly(ethylene-cooctadecene), chain dynamics were addressed, and which were quite unusual. The relaxation data at low temperatures indicates phase separation, while above $20^{\circ} \mathrm{C}$, a partially miscible blend is seen. $\mathrm{T}_{1 \mathrm{H}}$ data not shown indicates relaxation times of 650,280 , and $450 \mathrm{~ms}$ for the aPP, PE-OD and the blend, showing that 
that blend is intimately mixed at a length scale with an upper limit of $20 \mathrm{~nm}$ (as will be shown in the next paragraph). ${ }^{35}$
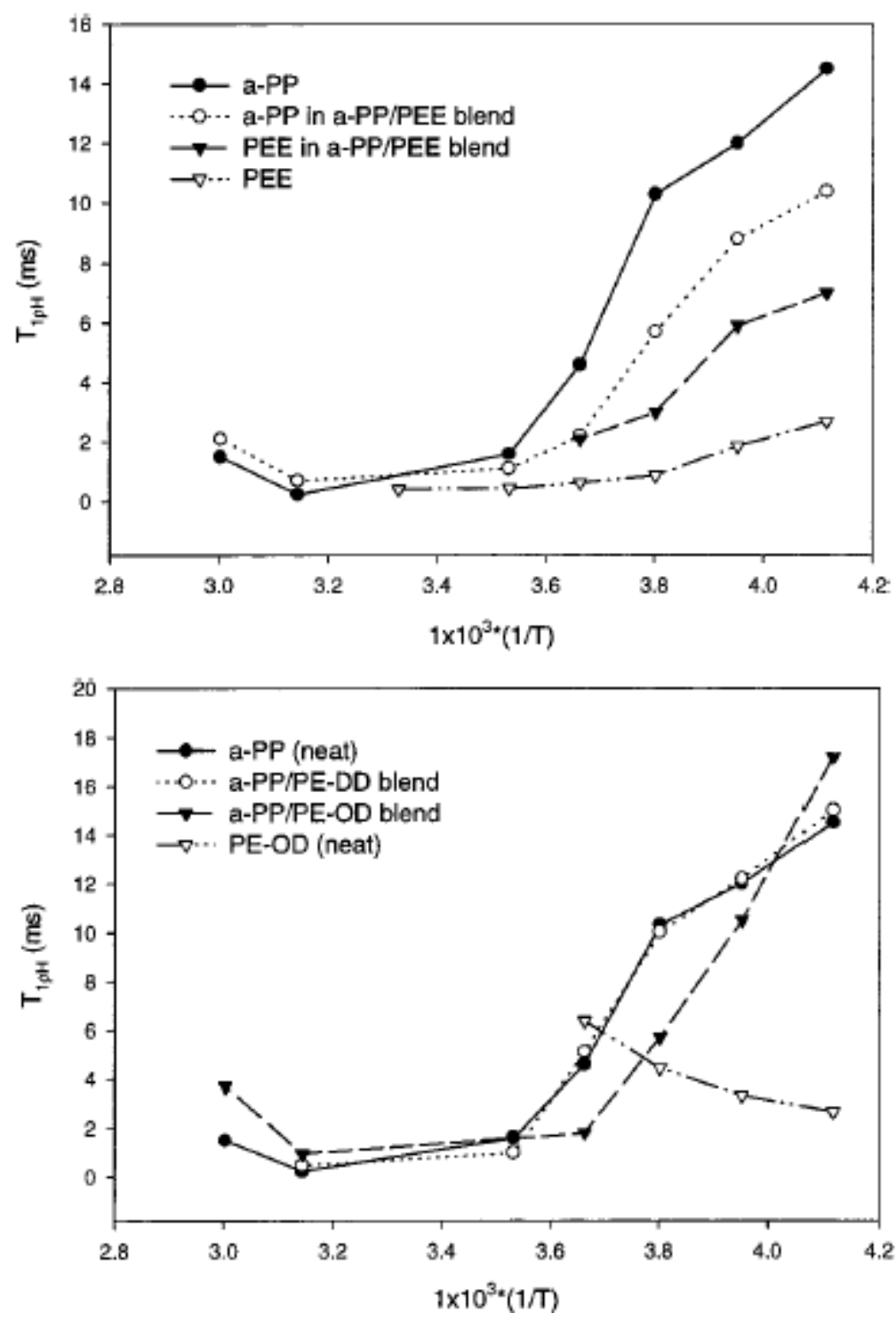

Figure 2-6 Variable temperature $T_{1 \rho H}$ plots for the components and the blends. Shown in (a) is aPP with PEE while in (b) aPP with PE-OD and PE-DD (taken from Ref. 35).

Relaxation in solid-state NMR allows a very quick approximation of the polymer blend degree of mixing with $\mathrm{T}_{1 \mathrm{H}}$ and $\mathrm{T}_{1 \rho \mathrm{H}}$ values determining upper and lower boundaries of the length scales of mixing, respectively. Using the equation 


$$
x=\sqrt{\frac{4 D \tau}{\pi}}
$$

either $\mathrm{T}_{1 \mathrm{H}}$ or $\mathrm{T}_{1 \rho \mathrm{H}}$ are used as the $\tau$ value. Here, $\mathrm{x}$ is the spin-diffusion distance while D is the diffusion coefficient. Typically, rough estimates of length scales of mixing can be determined by using spin-diffusion constants reported in the literature for similar blends if the exact diffusion coefficients are not known. As an example, White et al. reported $\mathrm{T}_{1 \mathrm{H}}$ and $\mathrm{T}_{1 \rho \mathrm{H}}$ values for PIB/PMS 50/50 blends, with which the upper length scale of $23 \mathrm{~nm}$ and lower length scale of $1.8 \mathrm{~nm}$ were established. Since the $\mathrm{T}_{1 \rho \mathrm{H}}$ measurements of the blend were in between the homopolymer values, but they did not converge to the same value, it was determined that this blend was homogeneous on a length scale in between $1.8 \mathrm{~nm}$ to $23 \mathrm{~nm} .{ }^{48}$

Instead of obtaining upper and lower limits on the length scales of mixing, the dipolar filter experiment allows for direct observation of the spin-diffusion process and hence, a much more precise evaluation of the length scale of mixing. ${ }^{33,35}$ The experiment is based on a series of phase shifted $90^{\circ}$ pulses where the number and the spacing between them are altered in such a way as to maneuver the magnetization so that only the mobile segments in a blend are selected, while the rigid components are lost. Here, the proton dipolar coupling is exploited so as to follow its strength in the blend. The longer the number of cycles in the $90^{\circ}$ pulse train and their spacing, as shown in Figure 2-7, the more selective is the sequence to mobile species, while the rigid ones with larger 
coupling are lost due to the cumulative action of dipolar coupling during $\tau$ periods. Once the polarization is established, a controlled spin-diffusion mixing time is introduced and polarization transfer from the mobile back to the rigid components is observed (until equilibrium is reached) if and only if spin-diffusion is operative. $^{33}$

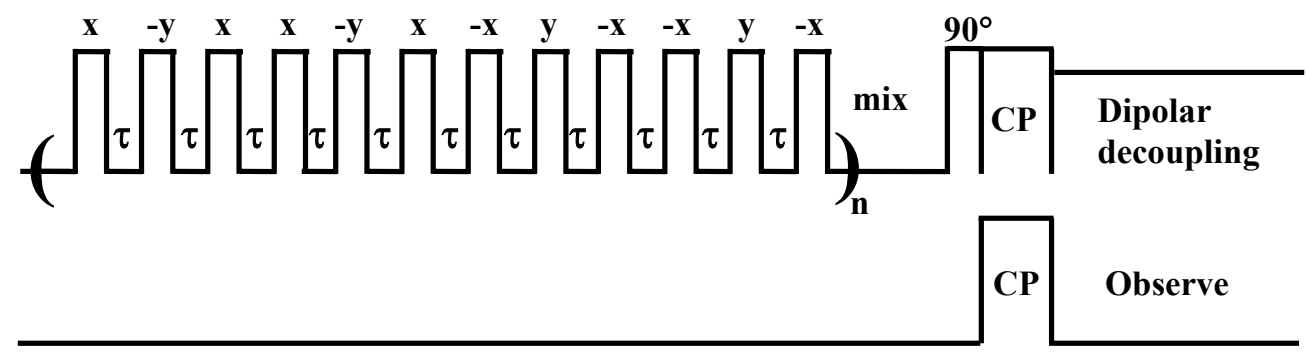

Figure 2-7 The dipolar filter pulse sequence where the train of $90^{\circ}$ pulses is followed by the crosspolarization time, and observation with dipolar decoupling. The $\tau$ (spacing in between pulses), $\mathrm{n}$ (number of loops) and mix (mixing time) can all be varied according to the blend requirements (taken from Ref. 33).

As an example, the spin-diffusion curves for neat aPP versus aPP in the aPP/PE-OD blend are compared, and it is immediately obvious that it takes roughly four times longer to achieve equilibrium in the blend than in the pure polymer. ${ }^{35}$ The pure polymer is only subject to intramolecular interactions, thus the additional source of polarization source in the blend must arise from the intermolecular spin-diffusion occurring between aPP and PE-OD chains. In order to quantify this data, the following equation is used:

$$
L_{a}=\frac{\varepsilon}{f_{a}} \sqrt{\frac{4 D \tau}{\pi}}
$$


where $\varepsilon$ is the number of orthogonal directions allowed for spin diffusion, $f_{a}$ is the volume fraction of phase A (for our 50/50 blends this will always be 0.5 ), $\mathrm{D}$ is the spin diffusion coefficient, and $\tau$ is the equilibrium mixing time (extrapolated from the initial part of the curve). The domain size for aPP/PE-OD is determined to be $5.1 \mathrm{~nm}$, falling in between the lower and upper limits of 2.1 and $20 \mathrm{~nm}$ as determined from the relaxation data. ${ }^{35}$ For this blend, partial miscibility is concluded due to the incomplete averaging of the relaxation data, but sensitivity to local dynamics is observed on length scales between $5.1 \mathrm{~nm}$ and $20 \mathrm{~nm}$. Another application of the same method was seen for the $\mathrm{PIB} / \mathrm{hhPP}$ blend, where a minimum domain size of $3.5 \mathrm{~nm}$ was established. ${ }^{33}$ Technical points which should be emphasized when utilizing this technique is the need for some difference in dynamics between the two polymers so that one can be selected over the other. Also, the blends should typically have LCST phase diagrams because with UCST phase behavior the temperature forming a miscible blend may be too high for efficient spin-diffusion. ${ }^{33}$

\section{2-4 Cross-Polarization with Magic Angle Spinning (CPMAS) NMR Experiment}

Cross-polarization with magic angle spinning and proton decoupling (CPMAS) was experimentally first realized by Schaefer and Stejskal in 1975

offering improved resolution and enhanced signal intensity in solid spectra. ${ }^{38,49}$ Solid state NMR has been instrumental in characterizing numerous rigid polymer systems because unlike in solution NMR, the polymers can be studied in their 
natural environment without exposing them to solvents, which could decompose or change their physical properties. The problem with solids is that line widths are large (several $\mathrm{kHz}$ ) due to the absence of rapid, isotropic molecular motion, which in solution NMR is responsible for the complete averaging of anisotropic interactions. $^{38,45,46,50}$ In addition to the severe spectral overlap, sensitivity problems also arise when studying low natural abundance nuclei such as ${ }^{13} \mathrm{C}$, making it difficult to acquire decent spectra due to the long relaxation times.

Schaefer and Stejskal successfully combined three techniques (high-power decoupling, MAS and cross-polarization) in order to simultaneously eliminate line broadening and increase sensitivity to allow studies of both structure and molecular motions present in rigid systems. In a system containing ${ }^{13} \mathrm{C}$ and ${ }^{1} \mathrm{H}$ nuclei, the major source of ${ }^{13} \mathrm{C}$ line broadening is the ${ }^{13} \mathrm{C}-{ }^{1} \mathrm{H}$ heteronuclear dipolar interaction ranging from $10-50 \mathrm{kHz}{ }^{45,50}$ The numerous interactions between the ${ }^{13} \mathrm{C}$ and abundant protons can be successfully suppressed by strong irradiation (decoupling) at the proton resonance frequency. Next, due to the absence of rapid tumbling, residual broadening due to the orientation-dependent chemical shift is also present. Chemical shift anisotropy can be successfully averaged out by mechanical rotation of the sample precisely at the magic angle $\left(54.733^{\circ}\right)$ at a rate fast compared to the anisotropy. ${ }^{51-53}$ This 'magic angle' allows the second Legendre polynomial $\left(3 \cos ^{2} \theta-1\right)$ to vanish, thus simplifying Equation 1-19. The rotation frequency has to be comparable to the CSA in order to get complete MAS averaging, because, if it is less, then the spectrum will contain sidebands centered on each isotropic shift spaced at multiples of $\omega_{\mathrm{r}}{ }^{38,45}$ The spinning speed and the 
CSA determine the number, intensity, and position of sidebands, and from this pattern, the full CSA can be mapped out. Loss of sensitivity and difficulty in spectral assignment come with the appearance of spinning sidebands, but methods do exist which will remove them.

The ${ }^{1} \mathrm{H}_{-}^{13} \mathrm{C}$ dipolar coupling, challenging because it causes line broadening, actually provides an opportunity for a sensitivity enhancement. The solution lies in cross polarization (CP) from the protons with high- $\gamma(267.512 \mathrm{rad}$ $\left.\mathrm{T}^{-1} \mathrm{~s}^{-1}\right)$ to carbons with low- $\gamma\left(67.264 \mathrm{rad} \mathrm{T}^{-1} \mathrm{~s}^{-1}\right)$, thus producing ${ }^{13} \mathrm{C}$ NMR signals

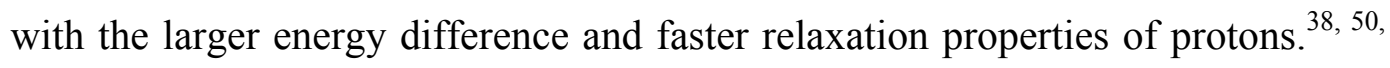
54, 55 Consequently, in this double irradiation rotating frame experiment, dilute carbon magnetization is built up with the abundant proton magnetization via the proton dipolar coupling that exists, as demonstrated by the cross-polarization spin-locking pulse in Figure 2-8 (a). The large energy difference seen in Figure 2-8 (b) in protons versus carbon levels does not allow the two nuclei to undergo energy conserving spin flips. Through spin-locking of the two systems in the rotating frame, the effective field of the protons is lowered and the HartmannHahn condition accomplished by irradiating the carbons with a specific radio frequency field strength. Now, the frequencies of both nuclei are matched according to $\gamma_{\mathrm{H}} \mathrm{B}_{1 \mathrm{H}}=\gamma_{\mathrm{C}} \mathrm{B}_{1 \mathrm{C}}$. $^{38,45,54}$ By matching the proton and carbon energy levels, or forcing the two spin systems to rotate at the same rate, the energy conserving spin flips between them are able to occur (Figure 2-8 (c)) ${ }^{38}$ Theoretically, the ${ }^{13} \mathrm{C}$ signal should be four times larger, and experimental pulse repeat time faster (on the $\mathrm{T}_{1 \mathrm{H}}$ timescale) allowing more scans to be acquired 
which will result in a large signal to noise improvement. ${ }^{45,46}$ Resolution gained by the CPMAS experiment can be destroyed by interference between coherent

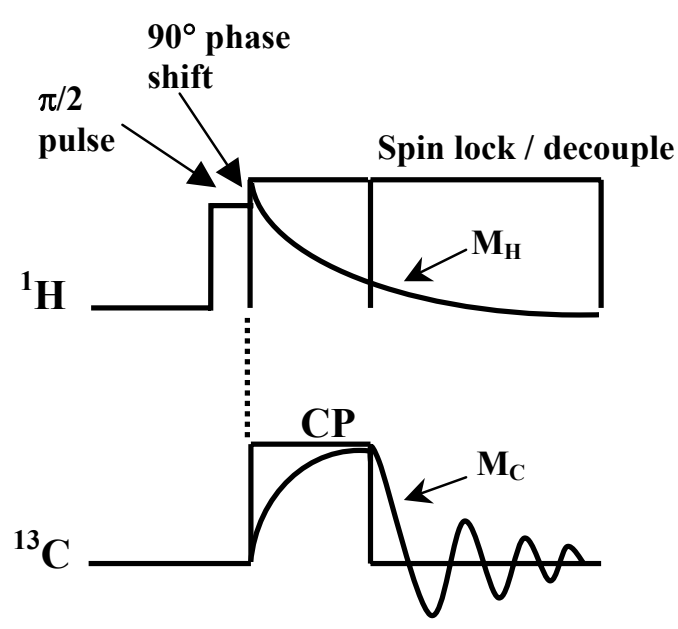

(a)

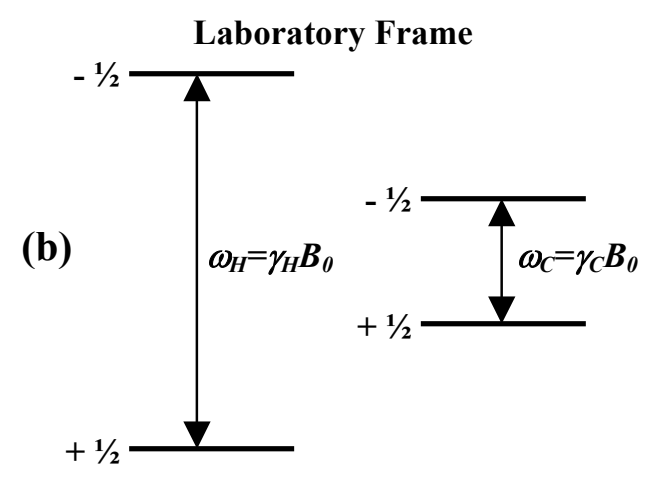

Doubly Rotating Frame $\left(\omega_{1 H}=\omega_{I C}\right)$

(c)

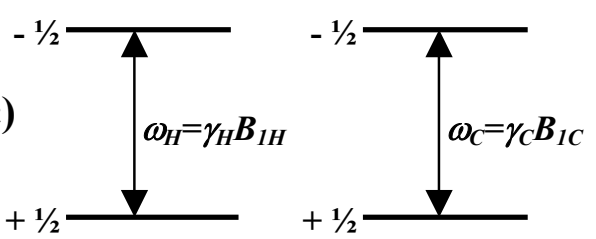

Figure 2-8 (a) ${ }^{1} \mathrm{H}-{ }^{13} \mathrm{C}$ Cross Polarization sequence and (b) thermodynamic energy levels before (top) and (c) after (bottom) the Hartmann-Hahn matching condition (taken from Ref. 38).

motion (spin-locking or sample rotation) and molecular motion, ${ }^{56-58}$ although these issues can be solved by variable-temperature (VT) CPMAS, as will be shown later. Nevertheless, the coherent averaging of the shielding anisotropy and proton irradiation produce narrow resonances at characteristic frequencies allowing for valuable dynamic and structural information to be gained. ${ }^{46}$ 


\section{Expansion of CPMAS to variable temperature}

The potential of CPMAS ${ }^{13} \mathrm{C}$ NMR spectroscopy was recognized early "as a highly diagnostic probe of physical and chemical properties in the solid state" and its directions expanded with the development of variable-temperature (VT) CPMAS. $^{38,} 46$ The sample temperature is changed by using the variable temperature air supply, which cools or warms the sample by blowing air on the middle of the rotor. A thermocouple positioned close to the rotor monitors the temperature. $^{44}$

The increased resolution capabilities of CPMAS combined with VT allows for studies of molecular structure, dynamics and reactivity in the solid state, for example looking at molecular motions and mechanisms with chemical exchange processes, molecular dynamics in polymers, and substances with unfavorable relaxation times $\left(\mathrm{T}_{1 \mathrm{p}}, \mathrm{T}_{1}\right)$ for cross-polarization at room temperature. $^{46}$ The variable temperature CPMAS experiment allows for manipulation of relaxation parameters and cross-polarization efficiency. For example, lowering the temperature of poly(ethylene oxide) to $-60^{\circ} \mathrm{C}$ (where the $\mathrm{T}_{1 \mathrm{pH}}$ is longer) allows for polarization transfer to occur and provides a clearly resolved ${ }^{13} \mathrm{C}$ spectra. For this and many other samples, the room temperature ${ }^{13} \mathrm{C}$ $\mathrm{CP}$ spectra are weak due to the $\mathrm{T}_{1 \mathrm{pH}}$ value being at its minimum. ${ }^{38}$ Thus, before cross-polarization has had sufficient time to occur, the proton order is destroyed. ${ }^{46}$ An important application of VT CPMAS is observing line shape changes as a function of temperature, by which chemical rate processes with lifetimes of $10-10^{-}$ ${ }^{6}$ s can be studied, because motions occur at rates comparable to the frequency 
separation of the signals. In the solid state a carbon pair, equivalent in solution due to rapid molecular reorientation, can be magnetically inequivalent, while at higher temperatures coalescence of the signal can be observed from the molecular motion making the atoms equivalent.

A vital part of variable temperature studies is progressive resonance broadening which occurs with stochastic (molecular) motion being present in a system and interfering with the coherent (applied rf fields) motion being introduced in the experiment. ${ }^{56}$ In their $\mathrm{T}_{2}$ theory, Rothwell and Waugh describe the loss in resolution caused by interference between the coherent motion, such as the decoupling frequency or sample rotation, and the incoherent polymer chain motion. ${ }^{57,58}$ The maximum amount of interference will be at the point where the $\tau_{\mathrm{C}}$ for a particular motion is equal to the frequency of decoupling or magic angle spinning. ${ }^{46}$ In the two extreme correlation limits, line widths are narrowed by different techniques. In the short correlation limit at high temperatures, $\omega_{1} \tau_{\mathrm{C}}<<1$, rapid motional averaging is responsible for line width reduction, while in the long correlation limit present at low temperatures, $\omega_{1} \tau_{\mathrm{C}}>>1$, the line width is reduced due to efficient decoupling of the $\mathrm{C}-\mathrm{H}$ dipolar interaction. Altering the temperature and mapping out the line-width regions can gain information regarding rotational rates and barriers. For example, various polymers with either rapidly reorienting side groups or backbones will experience these broadening effects at dissimilar temperatures as described by Rothwell and Waugh. The effect of broadening will change depending on the type of sample being studied; for example, a crystalline versus a glassy polymer has a homogeneous versus a 
heterogeneous environment, respectively. ${ }^{38}$ Therefore, a single correlation time for the crystalline sample will result in broadening at a specific temperature, while the glassy system will have the same effect spread over several temperatures. ${ }^{46}$

\section{2-5 Two-Dimensional ${ }^{13} \mathrm{C}$ MAS Exchange NMR Experiment}

One-dimensional solid-state NMR spectra may contain extensive overlap due to broad signals, which two-dimensional NMR overcomes by introducing a second frequency and demonstrates a large improvement in spectral resolution. ${ }^{50}$ Inhomogeneously broadened lines, caused by anisotropic chemical shift, dipolar or quadrupolar interactions, contain useful information regarding structure, dynamics and orientation of polymer segments. ${ }^{9,42}$ In particular, examining molecular motions of these materials via SSNMR, a connection between microscopic mobility and macroscopic mechanical properties can be established.

Experiments have been developed which unscramble complicated spectra by correlating various molecular properties or molecular states at different times, separating and recovering interactions, and determining geometrical and chemical connections. ${ }^{9,50,59}$ As Spiess says in his book, "Its interesting to note that even for a single site, the correlation of two inhomogeneously broadened patterns resulting from different interactions produces information not contained in the corresponding 1D spectra." ${ }^{\prime 9}$ More specifically, slow molecular motions (0.01$10 \mathrm{~Hz}$ ) and conformational exchange lifetimes between diverse molecular states can be studied using two-dimensional ${ }^{13} \mathrm{C}$ Exchange NMR experiments with MAS under slow spinning conditions. ${ }^{60}$ An advantage of this experiment is that it can 
be done at natural abundance, as opposed to other NMR methods capable of obtaining the same information, but demanding isotopic labeling. ${ }^{61}$

A generic two-dimensional experiment consists of four time periods including preparation, evolution, mixing and detection periods. As shown in Figure 2-9, the 2D-MAS exchange used in this work begins with the preparation period, where a nonequilibrium system is created via a $90^{\circ}$ pulse, together with a cross-polarization $(\mathrm{CP})$ pulse. The evolution period, $\mathrm{t}_{1}$, is where the spin system evolves under the influence of various nuclear spin interactions like chemical shift or dipolar coupling, and the signals are labeled with a precession frequency. During the mixing time $\left(t_{m}\right)$, which is a multiple of the rotor period $\left(t_{r}\right)$, there is a transfer of spin information among the different sets of spins due to molecular reorientations or chemical exchange. Detection takes place during $\mathrm{t}_{2}$, followed by several repetitions of the whole experiment while incrementing $t_{1}$ in order to complete the second dimension. The purpose behind a 2D exchange experiment is to determine whether there are any cross-peaks (off-diagonal intensities) connecting resonance frequencies from the evolution time $\left(\omega_{1}\right)$ with the detection time $\left(\omega_{2}\right)$. Two conditions can occur: (1) if the frequency before and after the mixing time are identical $\left(\omega_{2}=\omega_{2}\right)$, the signal will resemble a 1D spectrum and will be confined to the diagonal; (2) if the frequencies are different resulting from molecular reorientation or chemical exchange, cross-peaks will connect the various frequencies. ${ }^{9}$ An important aspect of the experiment is that the mixing time has to be a multiple of the rotor period in order for the CSA to be averaged out and artifacts removed. ${ }^{42}$ 


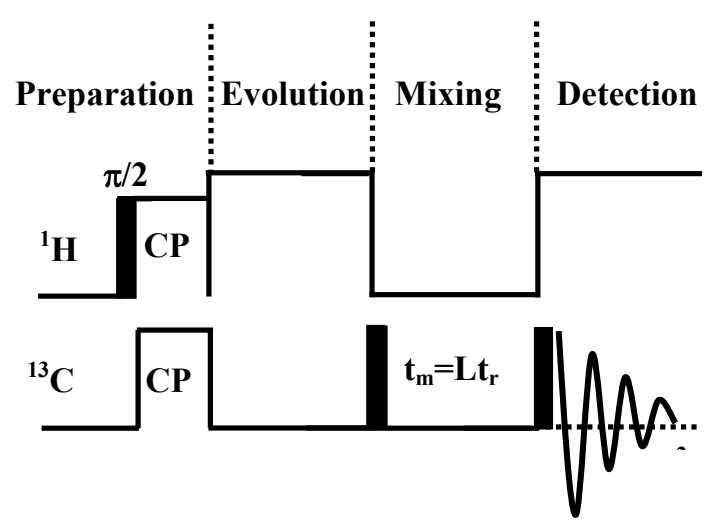

Figure 2-9 Pulse sequences demonstrating the $2 \mathrm{D}{ }^{13} \mathrm{C}$ MAS exchange experiment with four time periods labeled on top. The $90^{\circ}$ pulses are indicated in black, the cross-polarization (CP) and dipolar decoupling (DD) specified on each channel, and the mixing time $\left(\mathrm{t}_{\mathrm{m}}\right)$ shown as a multiple of the rotor period $\left(t_{r}\right)$ (taken from Ref. 9, 38).

An assortment of 2D exchange experiments exist, but our focus here is on the ${ }^{13} \mathrm{C}$ 2D Exchange MAS experiment in the slow spinning regime. ${ }^{62}$ By spinning fairly slowly, so both the isotropic and anisotropic components of the chemical shifts are present, both conformational and rotational chain dynamics can be studied in one experiment. An exchange of the isotropic chemical shifts (centerbands) demonstrates conformational transitions whereas an exchange of the anisotropic chemical shifts (sidebands) demonstrates rotational motion. ${ }^{42}$ Varying the mixing time permits the determination of the process correlation time, while changing the temperature establishes an activation energy. ${ }^{59}$

\section{D Exchange Experiments Around $T_{\mathrm{g}}{ }^{61}$}

Ultraslow chain dynamics involving conformational trans-gauche transitions were addressed near the $\mathrm{T}_{\mathrm{g}}$ for an amorphous polymer, atactic polypropylene (aPP) using high spectral resolution. Due to the sensitivity of solidstate isotropic chemical shifts observed by ${ }^{13} \mathrm{C}$ MAS NMR to molecular 
conformations and packing, trans-gauche transition can be easily identified for aPP, which has conformational contributions from sPP (syndiotactic) and iPP (isotactic) but various distributions of each. ${ }^{41,42}$ Various carbons and conformational splittings are identified in Figure 2-10 (a) and (b), where ${ }^{13} \mathrm{C}$ CPMAS spectra of aPP at two different temperatures are presented. Contributions from syndiotactic and isotactic polypropylene stereosequences (sPP and iPP respectively) to atactic polypropylene can be seen by the large distribution of the methylene signal, showing intramolecular conformational contributions. At the lower temperature $(250 \mathrm{~K})$, the conformations persist for a long time as seen with the splitting of $\mathrm{CH}_{2}$ (syndiotactic isotropic chemical shift of $\gamma$-trans $/ \gamma$-trans and $\gamma$ gauche/ $\gamma$-gauche at 48.6 and $40 \mathrm{ppm}$ and $\gamma$-trans $/ \gamma$-gauche from iPP at $44.5 \mathrm{ppm})$ and the methyl group. ${ }^{42}$ The $g g$ conformation is just a slight shoulder and is not clearly detected. In Figure 2-10 (b), the temperature is raised allowing conformational transitions between the $t t$ and $t g$, resulting in coalescence of the separated resonances from motional averaging.

Ultraslow polymer dynamics were observed above the $T_{g}$ of aPP $(239 \mathrm{~K})$ by monitoring the methylene group conformations as the temperature and mixing time were varied in the $2 \mathrm{D}$ exchange experiment. ${ }^{9,}{ }^{61}$ Below $\mathrm{T}_{\mathrm{g}}$ the conformation frozen on this time scale. The 2D exchange pattern in Figure 2-10 (c) at 250K, exchange is indicates conformational exchange for $\mathrm{CH}_{2}$ as well as the $\mathrm{CH}_{3}$ resonance for a mixing time of $500 \mathrm{~ms}$. Clear crosspeaks occurring between the $t t$ and $\operatorname{tg}$ methylene conformations are indicated on the contour plot. On the other hand, for a mixing time of $5 \mathrm{~ms}$, there is little exchange of the various 

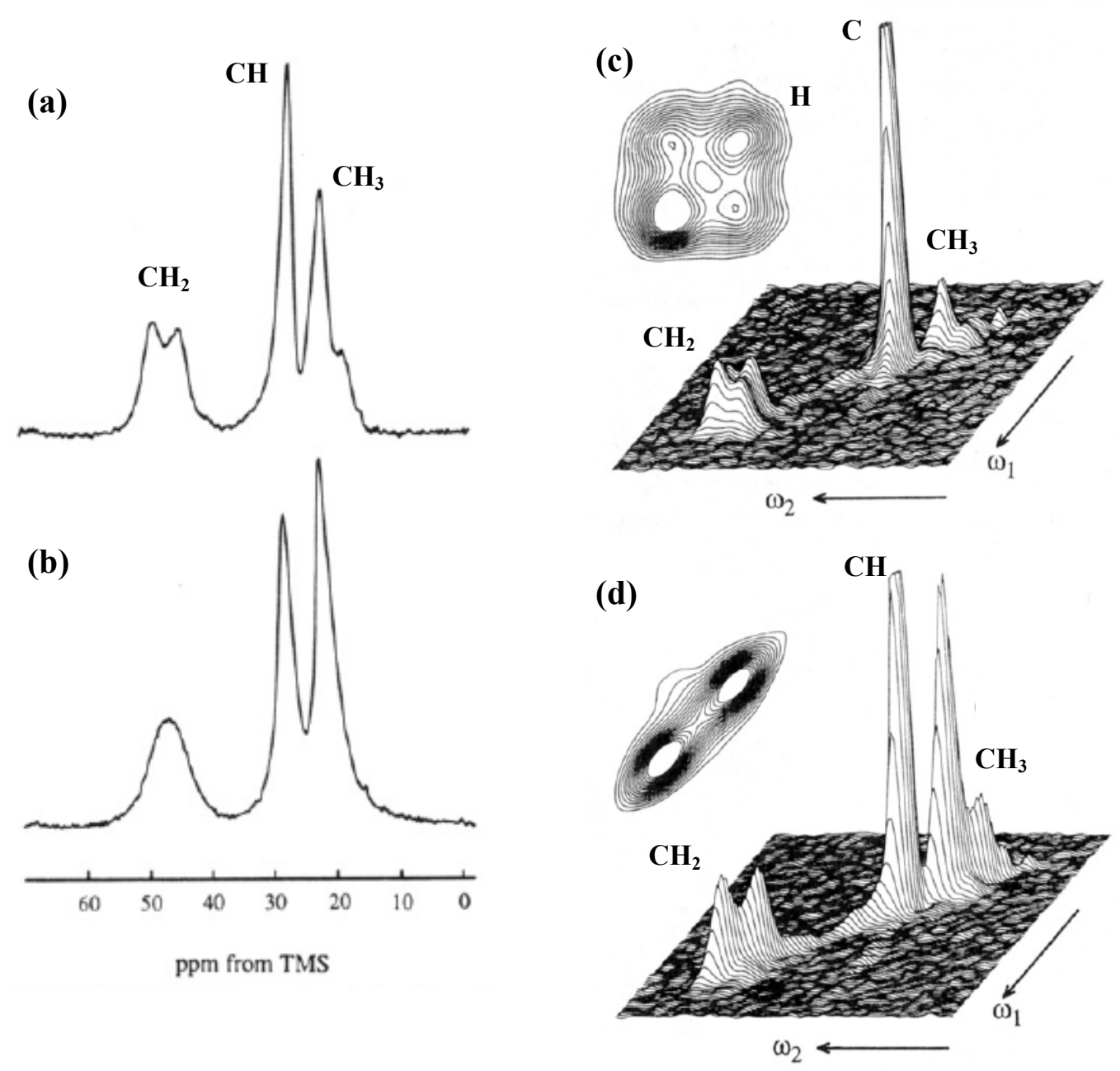

Figure 2-10 ${ }^{13} \mathrm{C}$ CP/MAS NMR spectra of aPP at (a) $\mathrm{T}=250 \mathrm{~K}$ and (b) $\mathrm{T}=262 \mathrm{~K}$ with spin rate of $3500 \mathrm{~Hz}$. Two-dimensional ${ }^{13} \mathrm{C} \mathrm{CP} / \mathrm{MAS}$ NMR spectra of aPP at $250 \mathrm{~K}$ with mixing times of (c) $500 \mathrm{~ms}$ and (d) $5 \mathrm{~ms}$ (taken from Ref. 28, 61).

conformational segments as indicated by the lack of off-diagonal intensity and lineshape representing the 1D spectrum. Extensive temperature 2D studies were completed to determine the average correlations times of exchange. As a result, it could be concluded that the conformational transitions observed are tightly coupled with the cooperative motion of the $\alpha$ process as determined by static $2 \mathrm{D}$ ${ }^{13} \mathrm{C}$ exchange NMR. ${ }^{61}$ 


\section{2-6 CODEX Description and Experimental Applications}

CODEX, Centerband-Only Detection of EXchange, is a one-dimensional MAS exchange experiment that allows for identification and characterization of slow motions on the order of $0.1 / \mathrm{s}$ to $10,000 / \mathrm{s} .{ }^{63,64}$ Molecular dynamics have typically been studied via multidimensional MAS NMR, exchange techniques; these are extremely informative but often suffer from poor resolution and sensitivity due to the requirements of anisotropically broadened lineshapes and strong sidebands. The 1D CODEX signal detection relies on signal decay from magnetization dephasing caused by slow motion during the mixing time $\left(t_{m}\right)$. High resolution spectra of polymers with small CSA's have already been demonstrated, proving to be easily attainable without isotopic labeling. ${ }^{65}$ CODEX provides an exciting, novel approach to the traditional exchange NMR methods generating site-specific fractions of slowly rotating groups, amplitudes of motion, correlation functions, and correlation times. ${ }^{66}$ CODEX will play a vital role in exploring polyolefin blends due to its high sensitivity to dynamic processes involving carbons with small CSA, maximum spectral resolution, no labeling requirement, and an ability to couple the sequence with preparation "filters", such as $\mathrm{T}_{1}, \mathrm{~T}_{1 \mathrm{p}}$, or $\mathrm{T}_{2}$.

The goal behind CODEX is to detect signal reduction if segmental reorientations take place during the mixing time, causing magnetization to dephase due to variations of the orientation-dependent chemical-shift frequencies. As seen in Figure 2-11, the experiment begins with a cross-polarization step during which the dilute carbon signal is enhanced by the abundant proton 


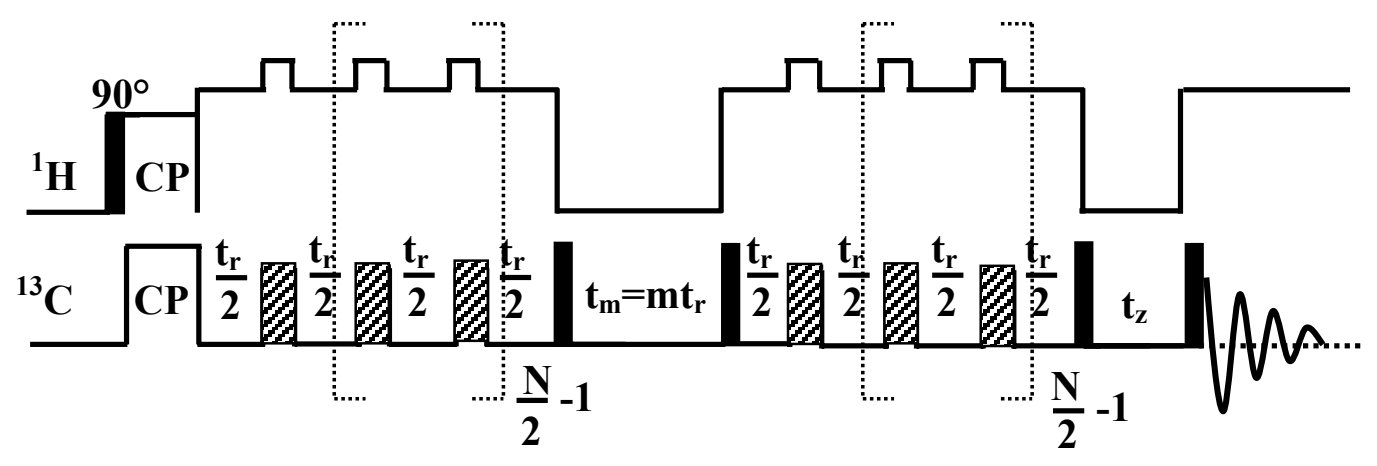

Rotor synchronization

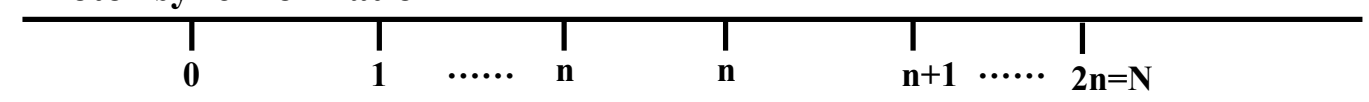

Figure 2-11 Pulse sequences for CODEX with rotor synchronization indicated on the bottom. The $90^{\circ}$ and $180^{\circ}$ pulses are indicated by black and dashed rectangles, respectively (taken from Ref. $64)$.

magnetization. ${ }^{64}$ In order to observe changes in the CSA during the $t_{m}$, the CSA, which is averaged out by MAS, is reintroduced during the recoupling period via two $180^{\circ}$ pulses per rotation period separated by spacings of half a rotor period. The mixing time, which is an integer multiple of the rotation period, $t_{r}$, is followed by a second CSA recoupling period and a second mixing time, $\mathrm{t}_{\mathrm{z}}$ (typically $\mathrm{t}_{\mathrm{z}} \cong \mathrm{t}_{\mathrm{r}}<1 \mathrm{~ms}$ ). Throughout the two mixing times, the magnetization is stored along the z-axis so no precession or dephasing takes place. At the end of the experiment, the combined time of both CSA recoupling periods is $\mathrm{Nt}_{\mathrm{r}}$ (each one being $\mathrm{Nt}_{\mathrm{r}} / 2$ where $\mathrm{N}=2,4,6, \ldots$ ) and, since dephasing is only a function of the total time $\mathrm{Nt}_{\mathrm{r}}$, high spinning rates can be used resulting in sideband-free spectra. ${ }^{63,}{ }^{64}$ The duration of $\mathrm{Nt}_{\mathrm{r}}$ is vital because the smaller the motional amplitude taking place in the sample, the longer time $\mathrm{Nt}_{\mathrm{r}}$ will be needed to produce significant dephasing. Consideration needs to be given to the carbon site 
CSA because the smaller the CSA, the less efficient the signal generation in the CODEX spectrum will be. Increasing the number of rotor cycles $\mathrm{N}$ can amplify the anisotropy to larger values. During the detection period either $\mathrm{CW}$ or ${ }^{1} \mathrm{H}$ TPPM decoupling is applied.

CODEX data is reported as a reference $\left(\mathrm{S}_{0}\right)$, CODEX $(\mathrm{S})$, and pureexchange spectra $\left(\Delta \mathrm{S}=\mathrm{S}_{0}-\mathrm{S}\right)$. The reference spectrum is performed to account for relaxation effects from $T_{1}$ and $T_{2}$ taking place during $t_{m}$ and $\mathrm{Nt}_{\mathrm{r}}$, respectively, resulting in a spectrum that will have the same relaxations as the CODEX, but no motion during mixing time. Consequently, the reference resembles the CODEX except for the interchange of the $t_{m}$ and $t_{z}$ times. Under the condition that no motion took place during $t_{\mathrm{m}}$ of the CODEX spectrum, all the chemical-shift evolution is refocused at the beginning of detection as seen in Figure 2-12(a). In this example, there is no decay of the signal intensity because the two evolution periods cancel each other out. If segmental motion occurred during $t_{m}$, the orientation-dependent frequency will be different before and after $t_{\mathrm{m}}$, preventing complete refocusing of the CSA as seen in Figure 2-12 (b). The pure-CODEX indicated is obtained by subtracting the CODEX intensity $\mathrm{S}=\mathrm{S}\left(\mathrm{t}_{\mathrm{m}}, \mathrm{Nt}_{\mathrm{r}}\right)$ from the reference $\mathrm{S}_{0}=\mathrm{S}\left(0, \mathrm{Nt}_{\mathrm{r}}\right)$. As a result, the only signal left behind in the pureexchange CODEX $\left(\Delta \mathrm{S}=\mathrm{S}_{0}-\mathrm{S}\right)$ consists entirely of slowly-exchanging sites. Normalized CODEX intensities plotted against $t_{m}(m s)$ provide correlation functions of the dynamics present. ${ }^{66}$ To simplify quantitative analysis, the immobile site intensity is kept constant, while normalized CODEX intensities 
plotted against $\mathrm{Nt}_{\mathrm{r}}$ offer amplitudes of slow molecular motion that took place during $\mathrm{t}_{\mathrm{m}}$.

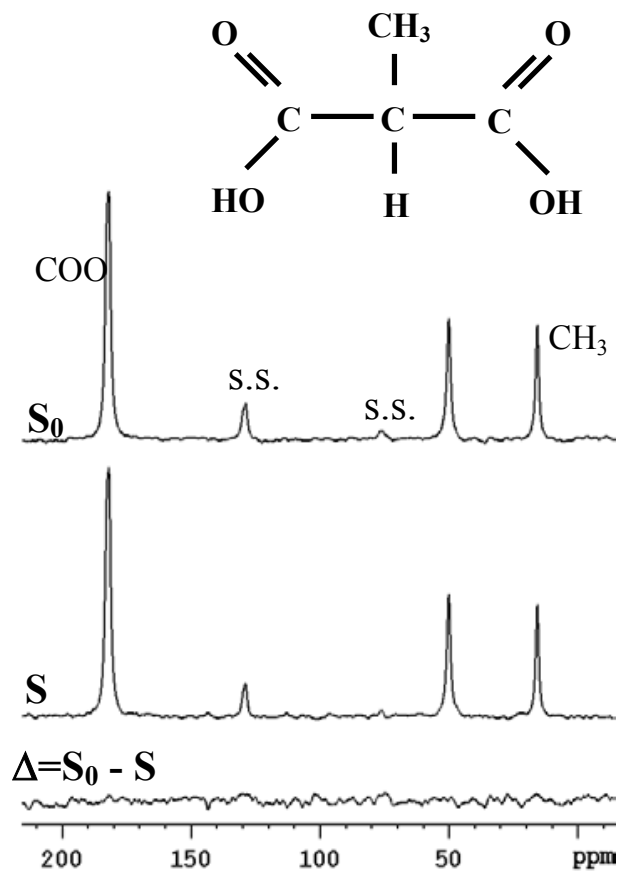

(a)

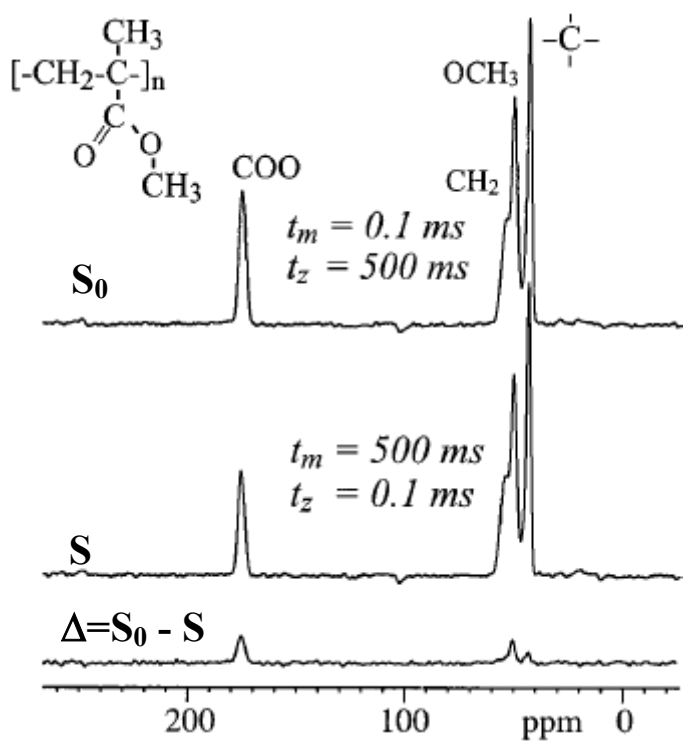

(b)

Figure 2-12 (a) Three spectra showing the reference, CODEX, and pure-exchange for methylmalonic acid at room temperature $\left(\mathrm{t}_{\mathrm{m}}=200 \mathrm{~ms}, \mathrm{t}_{\mathrm{z}}=1 \mathrm{~ms}\right.$ and $\left.\mathrm{Nt}_{\mathrm{r}}=500 \mu \mathrm{s}\right)$ (repeated data from S1999 paper to confirm in our pulse program was functional). (b) Differential signal for the pureexchange of aPMMA (amorphous poly(methyl methacrylate)) indicating slow-exchange taking place $\left(\mathrm{t}_{\mathrm{m}}=500 \mathrm{~ms}\right.$ and $\left.\mathrm{Nt}_{\mathrm{r}}=800 \mu \mathrm{s}\right)$ (taken from Ref. 64, 66).

Some technical aspects and potential problems of the experiment should be emphasized. As seen from the pulse sequence, the experiment is rotor synchronized (Figure 2-11), thus stable spinning and a clear trigger mark on the rotor are essential. As reported by Schmidt-Rohr, 4- $\mu$ s timing errors could produce significant artifacts and could be detrimental to signal amplitude measurements. ${ }^{63}$ Spectrometer drift is also taken under consideration; typically, 
the reference and the CODEX experiments are run in blocks by switching between the two acquisition modes every five minutes (block averaging). Pulse imperfections are accounted for in phase cycling according to the xy- 8 scheme. A severe limitation of the CODEX is that very fast motions are not detected, but this is where other experiments, such as ${ }^{2} \mathrm{H}$ quadrupolar line shapes or relaxation, can be substituted. ${ }^{63}$

The original CODEX publication, demonstrated the utility of the pulse sequence on several samples such as methylmalonic acid (MMA), dimethyl sulfone (DMS) and poly(methyl methacrylate) (PMMA) (Figure 2-12). ${ }^{64}$ The unlabeled MMA, a crystal at room temperature, is used as a reference since the pure-exchange CODEX MMA spectrum show no intensity (reference signal minus CODEX signal show a null) indicating no slow motion. ${ }^{64}$ On the other hand, the authors demonstrated both large-amplitude side group motions as well as smaller amplitude backbone motions for unlabeled amorphous PMMA. Here, CODEX demonstrates its superiority because previous studies on PMMA dynamics not only required multidimensional NMR studies but also, expensive ${ }^{13} \mathrm{C}$ and ${ }^{2} \mathrm{H}$ labeling. The authors mapped out the CODEX exchange intensity with mixing time to determine correlation times and functions. For example, by comparing the experimental curve with simulations of the several possible angle reorientations for DMS, small and large amplitude motions of $70^{\circ}$ and $110^{\circ}$ were determined.

Many other applications of the CODEX pulse program are appearing in the literature. ${ }^{65,}$ 67-69 An interesting example of polyolefin dynamics was 
established by Schmidt-Rohr in 2000. As seen in Figure 2-13(a), CODEX was run on a mixture of DMS and $\mathrm{PB}$ at roughly room temperature, and at short mixing times, only DMS demonstrated slow motion as indicated by the differential signal in the pure-exchange spectrum. Upon lowering the temperature

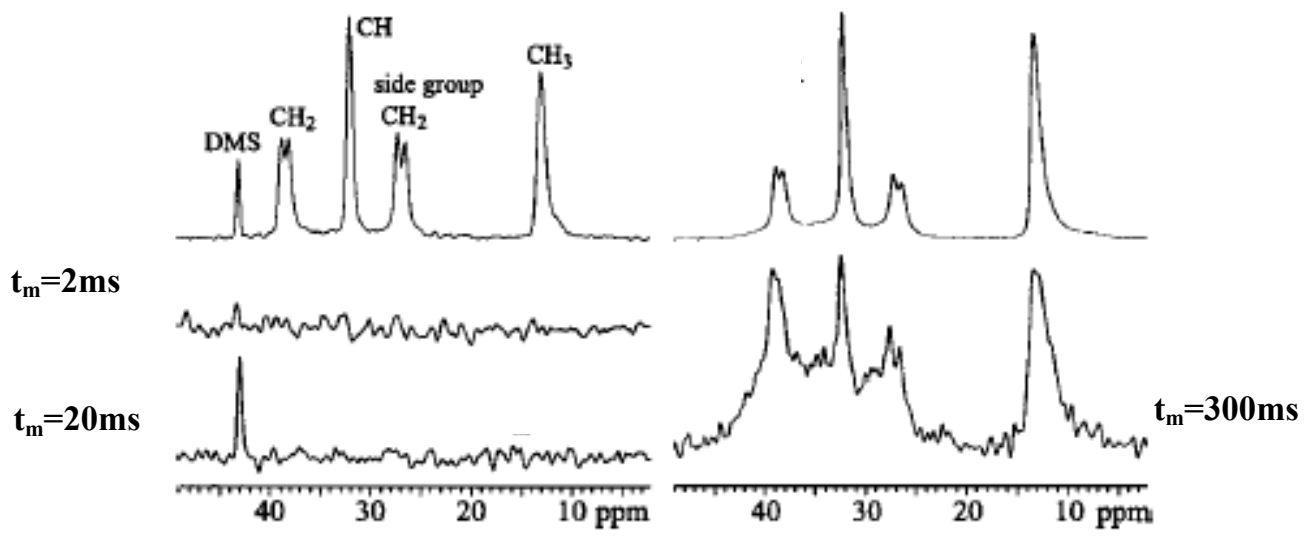

(a)

(b)

Figure 2-13 (a) DMS mixed with iPB spinning at $6 \mathrm{kHz}, \mathrm{Nt}_{\mathrm{r}}=667 \mathrm{us}$ and $\mathrm{T}=293 \mathrm{~K}$. (b) iPB at $260 \mathrm{~K}$. Both figures show reference spectra on top and pure-exchange spectra below (taken from Ref. 63).

below the $\mathrm{T}_{\mathrm{g}}$ of $\mathrm{iPB}$, the slowly moving segments of the amorphous domains are selected. The broad signal in the baseline of the crystalline dominated reference spectrum at the top of Figure 2-13 (b) is from the conformationally disordered amorphous regions, and becomes clearly observable following the subtraction of reference minus CODEX.

The pulse sequence specifications have been confirmed in our laboratory by focusing on rotor synchronization and block averaging by means of replicating literature data. As already seen in Figure 2-12, crystalline MMA was verified to have no signal in the pure exchange, for which no motion should occur. Next, differential molecular dynamics were detected on crystalline bisphenol-A $\left(4,4^{\prime}-\right.$ 
isopropylidenediphenol) following our recent investigations of ring dynamics. ${ }^{70}$

Figure 2-14 shows the normalized pure-CODEX results plotted versus mixing
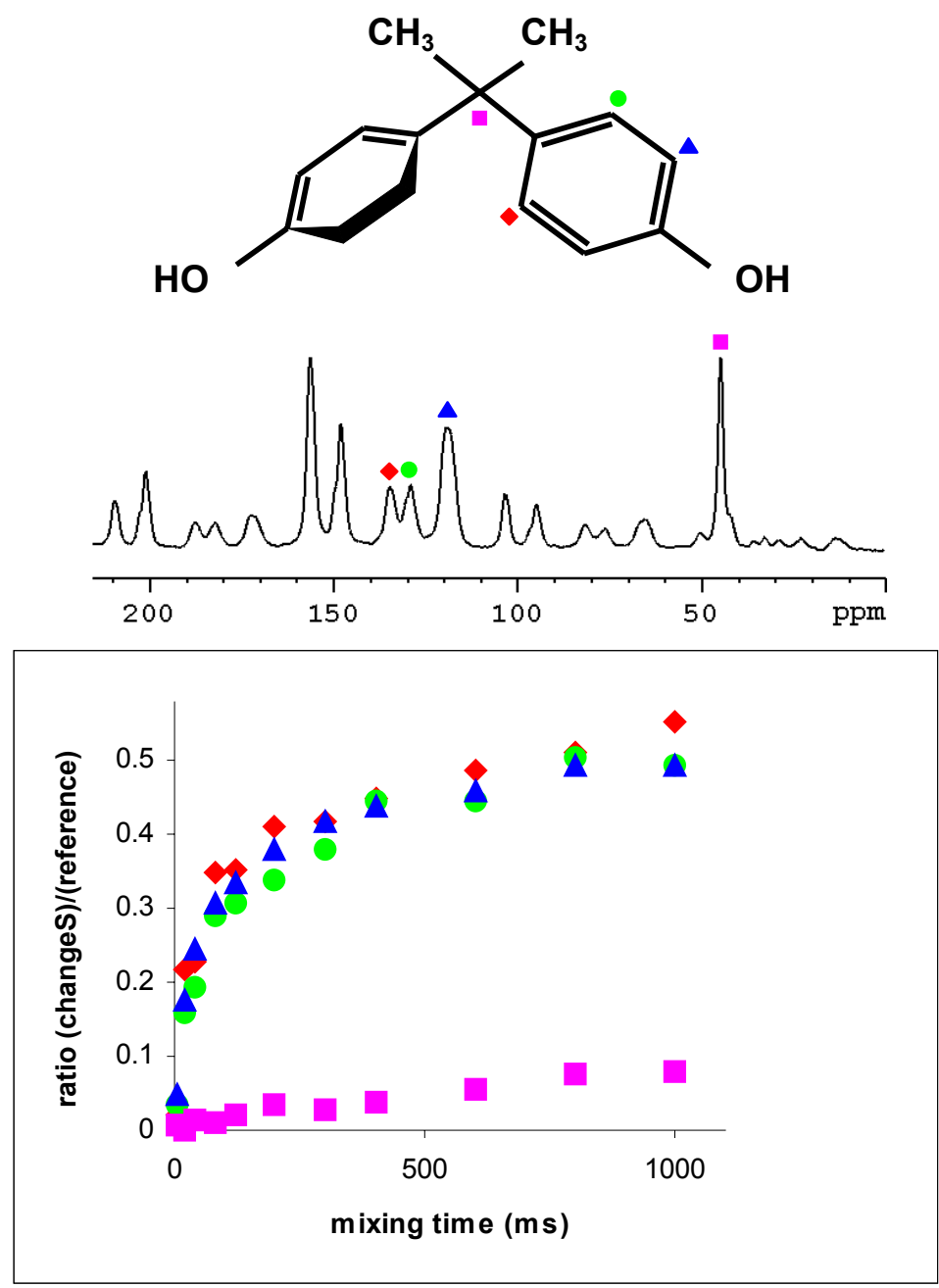

Figure 2-14 Pure CODEX exchange intensities as a function of mixing time for aliphatic quarternary $(\square)$ and protonated aromatic $(\bullet, \nabla, \mathbf{\Delta})$ carbons in BPA (structure above). Data were obtained at $308 \mathrm{~K}$ and using $4 \mathrm{kHz}$ MAS.

time for different BPA carbons. It shows that aromatic rings reorient with a tens of hertz correlation time, in agreement with our recently published 2D exchange data. Also, the plot agrees with BPA CODEX data reported by Schaefer, where long-time motions were observed for the backbone quaternary carbon. Thus, the 
availability of dynamics data for multiple peaks from both polymers in our blends (at high resolution) makes the CODEX an ideal tool since the total information content is enhanced relative to the $2 \mathrm{D}$ approach (even though the type of motion is more apparent from the 2D). Data has already been acquired at temperatures both above and below the blend critical solution temperatures.

Lastly, two-dimensional exchange NMR and CODEX techniques can be combined to study molecular dynamics. Since CODEX under MAS is sensitive to nuclei such as ${ }^{13} \mathrm{C}$ and ${ }^{29} \mathrm{Si}$ at natural abundance, it can be used as a preliminary approach to identify sites with certain dynamics, correlation times and motional amplitudes. $^{63}$

\section{2-7 Deuterium NMR Studies and Simulations}

Static deuterium NMR using quadrupolar echo experiments combined with lineshape simulations are an excellent probe for dynamic process in the solid state. $^{38,50,71}$ Molecular dynamic information is concealed in deuterium lineshapes, and unraveling the lineshape can reveal the type and time scale of the particular molecular motion. The difficulty of acquiring specifically labeled polymers due to deuterium's low sensitivity, profits by site-specific sensitivity to the nature, the amplitude and rates of molecular motions. ${ }^{59}$ Deuterium $\left({ }^{2} \mathrm{H}\right)$, with a spin of 1 and a small electric quadrupole moment has a quadrupole coupling constant $(\chi)\left(=\mathrm{e}^{2} \mathrm{qQ} / \mathrm{h}\right)$ around $200 \mathrm{kHz}$, and has been used often in lineshape motional studies, relaxation and exchange experiments for solids. ${ }^{50}$ Pake patterns occur for powder spectra of static samples, with the horns split by $3 / 4 \chi$ arising from the two 
possible spin transitions. These methods are well suited for solid-studies, due to the sensitivity of deuterium line shapes to motion with correlation times from $10^{-4}$ to $10^{-6} \mathrm{~Hz}^{59}$

Large widths of ${ }^{2} \mathrm{H}$ powder (static) spectra, means that high powered pulses need to be used in order to preserve the signal, and as a result, there is a requirement for a dead time to allow the electronics to recover. ${ }^{71}$ During the short dead time, the FID quickly decays and a specific echo method is required to recover the 'lost' signal. By recovering the signal, no intensity is lost from the parallel edges of the powder pattern, and the acquired signal is undistorted. ${ }^{38}$ As demonstrated in Figure 2-15, a quadrupole echo pulse sequence is generally used, in order to overcome the problems of the receiver dead time. The sequence

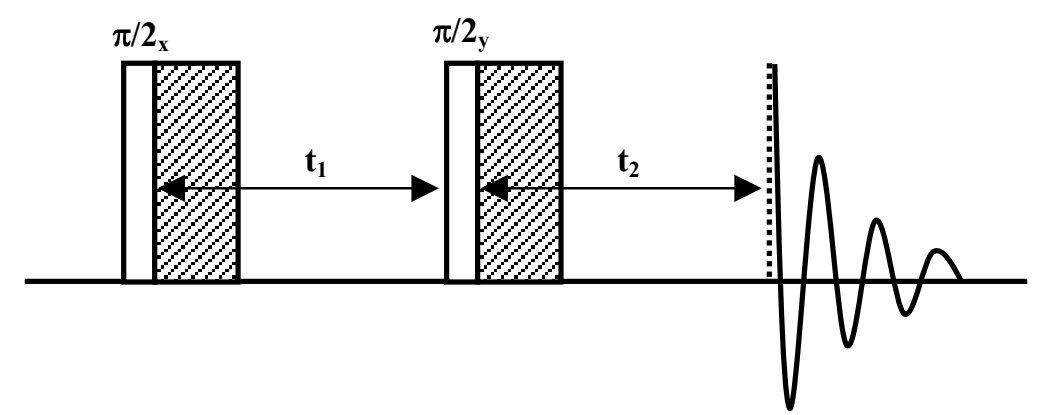

Figure 2-15 Quadrupole Echo pulse sequence where the $\pi / 2$ pulses are indicated in white, recovery time is dashed, and $t_{1}$ and $t_{2}$ are the two delays as indicated (taken from Ref. 38).

consists of two $\pi / 2$ pulses with a phase shift and two delay periods. At the end of the $t_{2}$ period, the inhomogeneously broadened lines are refocused through the echo. As seen from the pulse sequence, the two delays $t_{1}$ and $t_{2}$ are equal, but in practice, $\mathrm{t}_{2}$ is adjusted in order to begin the data acquisition exactly at the echo maximum. To establish the echo maximum, the receiver phase must be adjusted 
to obtain a pure absorptive phase. Also, due to the digitized data appearing on the computer screen, many times the data acquisition is started prior to $2 \tau$ so that the true echo can be located. Furthermore, the central part of the powder pattern needs to be on resonance in order to achieve uniform excitation over the entire powder pattern with the short, high powered pulses, and once on resonance, the match setting on the probe can be adjusted. ${ }^{71}$

Information about the rates and mechanisms of dynamic processes can be studied by sequentially changing components in the quadrupolar sequence, ${ }^{50,72}$ such as a representative deuterium study of molecular motion via echo intensity as a function of $t_{1}$. Results of the echo intensity as a function of $t_{1}$ together with the powder lineshape indicate useful information on the rate and geometry of molecular motional processes. In addition, the temperature dependence of the processes can be observed. By exploiting the temperature dependence of quadrupolar echo spectra, slow to fast exchange processes can be analyzed via adjusting the rates of dynamic processes. Some representative spectra of particular molecular motions are plotted in Figure 2-16. By comparing these theoretical lineshapes to the experimental, the types of motions present in the material can be predicted. Furthermore, typical deuterium studies are accompanied by spectral simulations, as will be demonstrated in the following example. $^{72}$

An excellent combination of experimental quadrupolar echo data with simulations is demonstrated by Simpson et al. on films of poly(p-phenylene vinylene) $\left(\mathrm{PPV}-\mathrm{d}_{4}\right)$ with deuteration on the phenylene ring positions as shown in 
(a) Static C-D
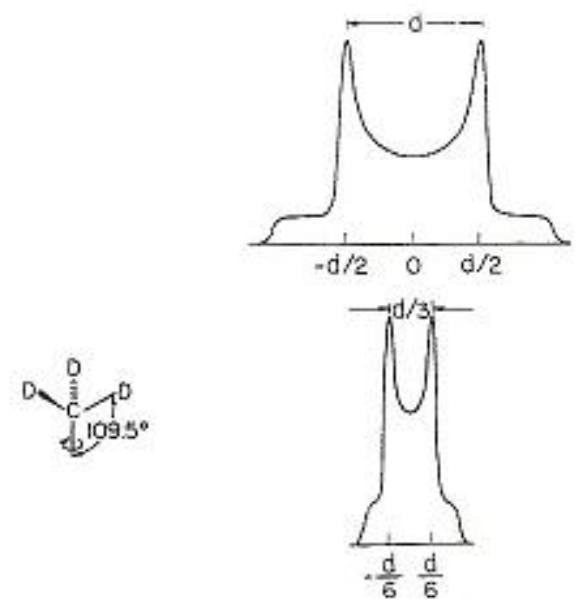

(b) Rotating Methyl

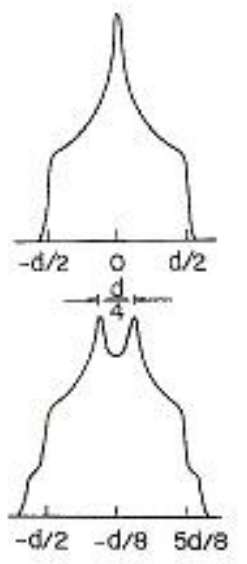

(d) $180^{\circ}$ Phenyl Ring Flip
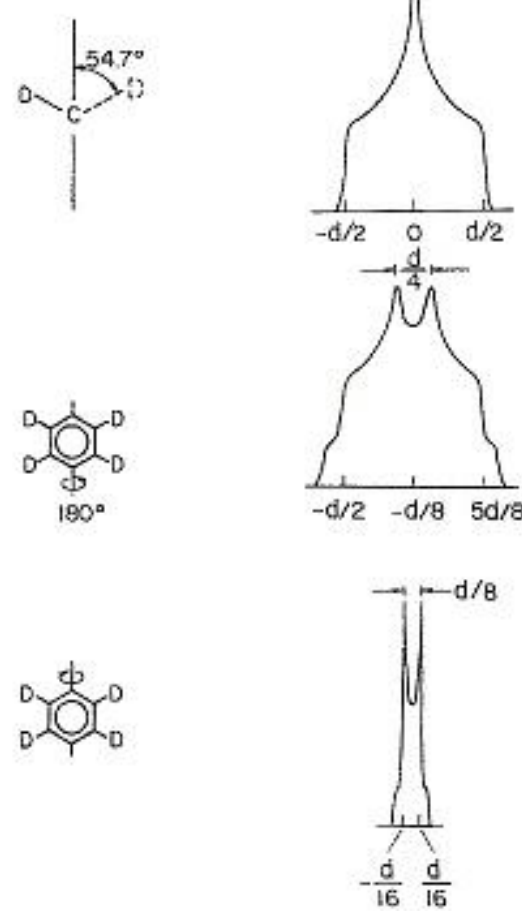

Figure 2-16 Theoretical ${ }^{2} \mathrm{H}$ NMR lineshapes of several anisotropic motions (taken from Ref. 38).

(e) Free Diffusion of Phenyl Ring

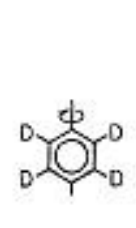

Figure 2-17 (a). ${ }^{73}$ As described in the paper, all phenylene rings undergo $180^{\circ}$ jumps about the 1,4 axis causing the static spectrum to be motionally averaged by the jumps as indicated in part (b). A temperature study using the quadrupolar echo pulse sequence, as seen in Figure 2-17 (c), shows that the jump motion is thermally activated with an activation energy of $15 \mathrm{kcal} / \mathrm{mol}$. From the 
temperature dependence it can be seen that at higher temperature, there is no trace of the static component. Simulations based on the model of thermally activated rotational jump motions is shown in Figure 2-17 (d), with the corresponding

(a)

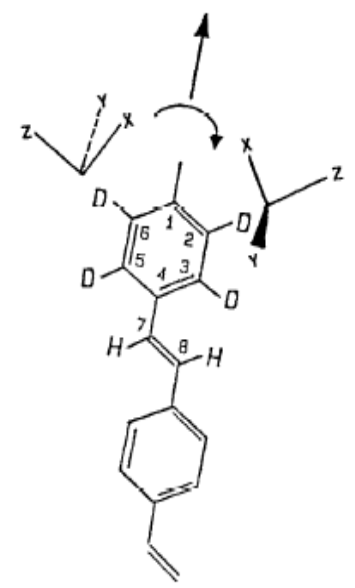

(b)

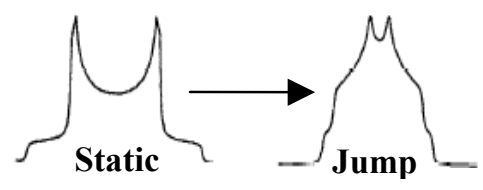

(c)

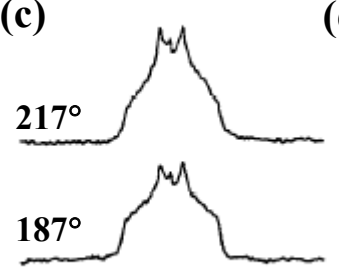

(d)
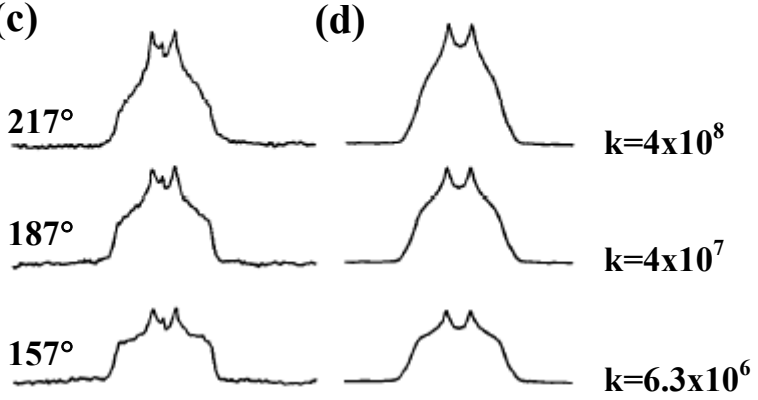

$127^{\circ}$
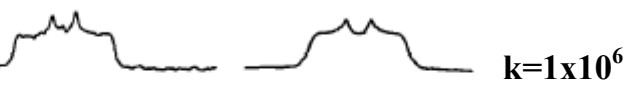

$97^{\circ}$
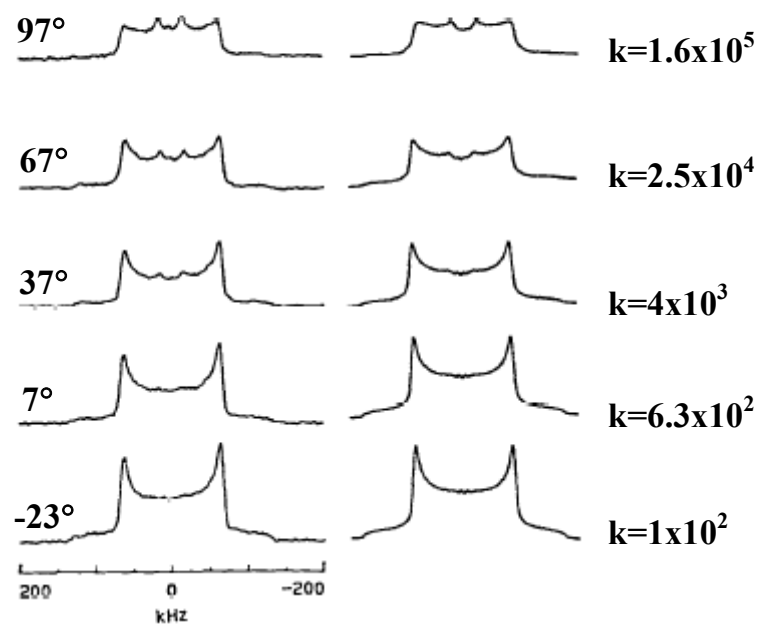

Figure 2-17 (a) The structure of PPV- $\mathrm{d}_{4}$ with indicated deuteration sites. (b) A static spectrum transitioning to a motionally averaged and axially asymmetric jump spectrum. (c) A variable temperature stackplot of ${ }^{2} \mathrm{H}$ quadrupole echo spectra with the simulations indicated in (d) (taken from Ref. 73).

rates. Other experiments, including that variable temperature $T_{1}$ measurements also support the model of $180^{\circ}$ jumps. This work clearly shows how deuterium NMR has been utilized to study molecular motions in numerous materials. ${ }^{73}$ 


\section{2-8 Xenon NMR Background}

${ }^{129} \mathrm{Xe}$ nuclear magnetic resonance spectroscopy is a useful technique for characterizing the morphologies of polymer systems such as polymer blends and copolymers. Direct phase differences can be seen by xenon resonances in slow and fast exchange between phases, domain sizes can be estimated if diffusion coefficients are known, and homogenous versus heterogeneous environments can be determined by the number and the line shapes of xenon resonances in the spectrum. $^{74}$ It is an excellent NMR probe because of the high natural abundance (26\%), high magnetogyric ratio, relative inertness under ordinary conditions, and minimal disruption of the structure being probed. ${ }^{75}$ The xenon atom is non-polar, has a small van der Waal's radius $(2.2 \AA)$, and it is soluble in polymers. The xenon atom has a large, highly polarizable electron density, making the chemical shift very sensitive to small changes in the electronic environment and temperature alterations. The xenon chemical shift (directly proportional to the collision rate) will reflect the polymer free volume or density, with larger free volumes showing smaller chemical shifts. $^{76}$

A single xenon NMR line for a polymer blend means that the blend is either miscible or that the blend has small domains with rapid xenon diffusion between the chemically distinct phases, as defined by the NMR time scale. The rapid chemical exchange taking place actually collapses the two different resonances into a single line. Miscibility can be distinguished from chemical exchange by the temperature dependence of solubility and xenon chemical shift in 
polymers. The xenon chemical shift in a miscible blend is averaged over the free volume sites available.

Above $T_{g}$, xenon rapidly diffuses between sites because of fluctuations in the polymer segment density. Rapid diffusion collapses broad lines to narrow

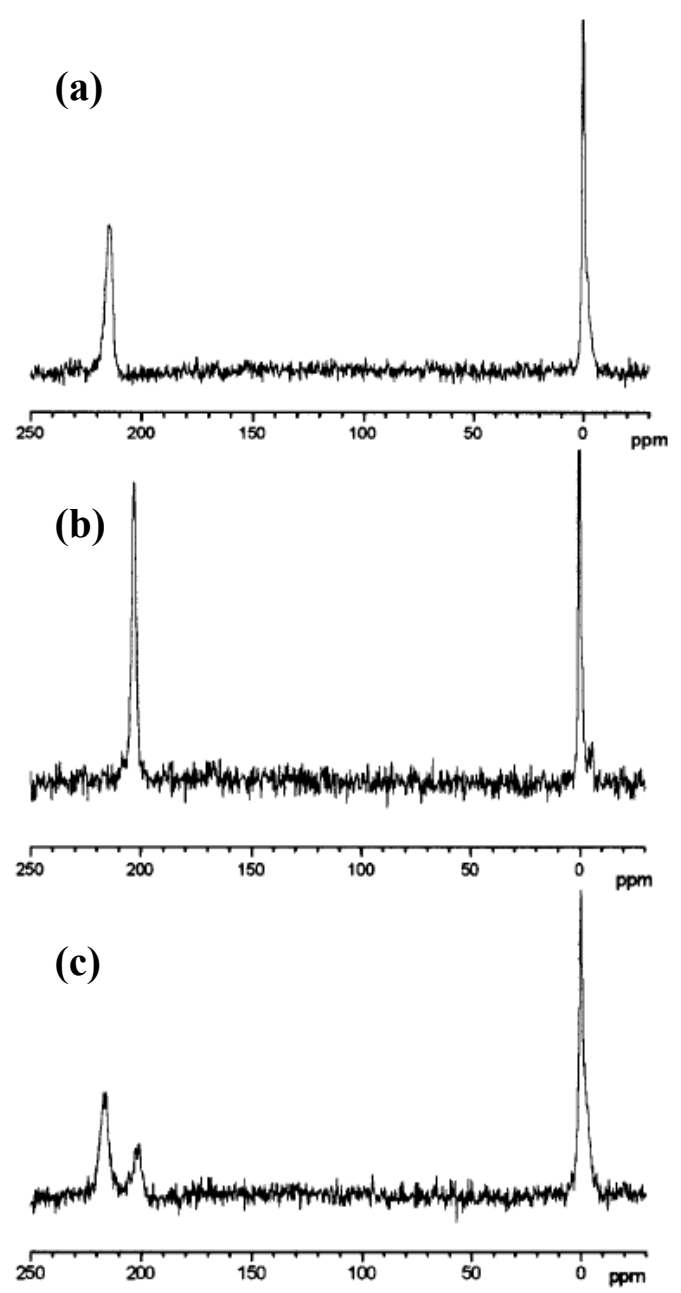

Figure 2-18 (a) ${ }^{129} \mathrm{Xe}$ NMR spectrum of PP (b) EPDM and (c) blend of PP/EPDM (80\%/20\%) (taken from Ref. 77).

ones. At temperatures above $\mathrm{T}_{\mathrm{g}}$, a single narrow resonance is seen for a homogenous phase and multiple resonances for heterogeneous phases as seen in 
Figure 2-18(a) for an incompatible blend of EPDM/PP (elastomeric terpolymer ethylene-propylene-diene with a semicrystalline polypropylene). Below $T_{g}$, the polymer chain motion ceases, causing the xenon to be trapped in various sites. Consequently, there is a distribution in local free volumes and inhomogeneously broadened lines. Thus resolution is lowered due to the increased line width, and sensitivity lowered due to decreased solubility of xenon gas.

A representative xenon spectrum can be observed in Figure 2-18. As seen above, the ${ }^{129} \mathrm{Xe}$ spectrum of the blend has a free xenon peak at 0ppm that serves as a reference, as well as two peaks representing the xenon in the EPDM domains (202ppm) and in the PP matrix (218ppm) corresponding to the pure component shifts. The xenon $T_{2}$ is much smaller in the blend from the fast exchange between the smaller domains, thus the line widths are larger. The experimental techniques for these experiments are simple, where high-pressure NMR tubes are loaded with the samples and evacuated for a period of time. Defined amounts of xenon gas are transferred into the tubes after which they are sealed off under vacuum and allowed to equilibrate. ${ }^{77,78}$

The small xenon molecule has advantages over other techniques in probing blend morphologies. When probing blend morphologies, there is a marked resolution advantage of ${ }^{129}$ Xe NMR over a method such as DSC. The timescale of separating different domains is revealed by lineshape collapse (Figure 2-19(a)), rather than detecting changes in the slope of a DSC graph (Figure 2-19(b)). Walton et al. demonstrated this with their blend of polyisoprene and polybutadiene as seen in Figure 2-19. They were able to demonstrate that 
(a)

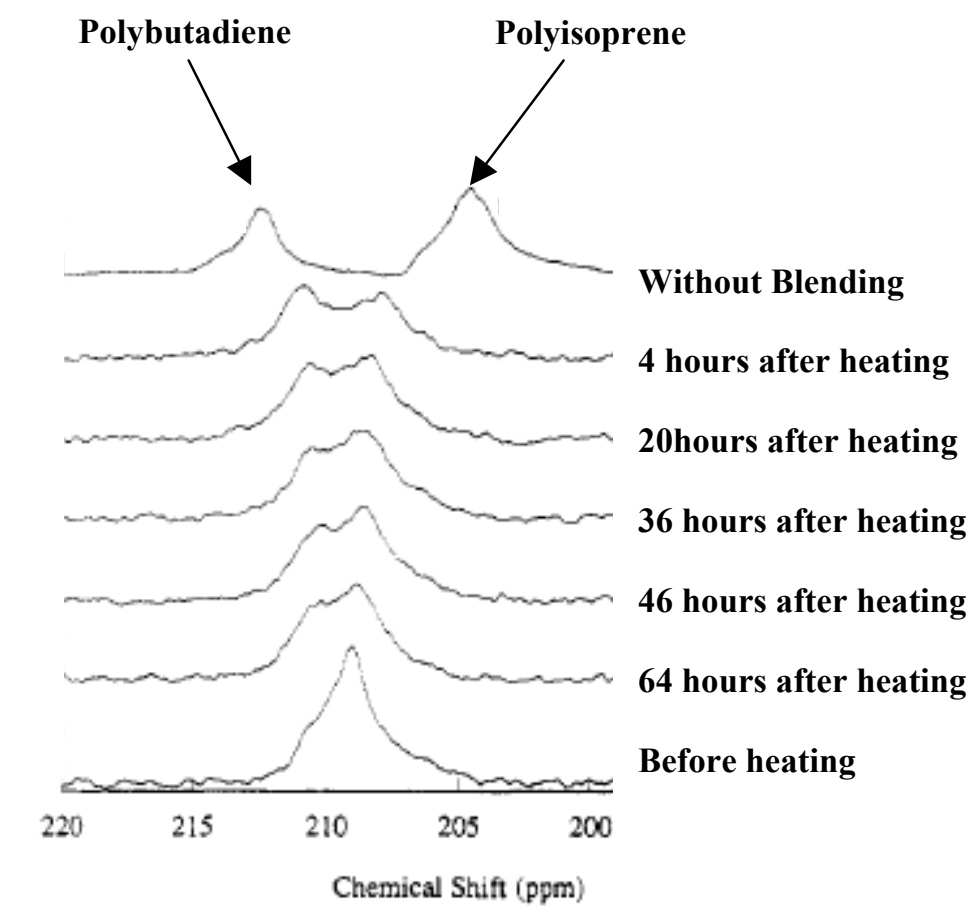

(b)

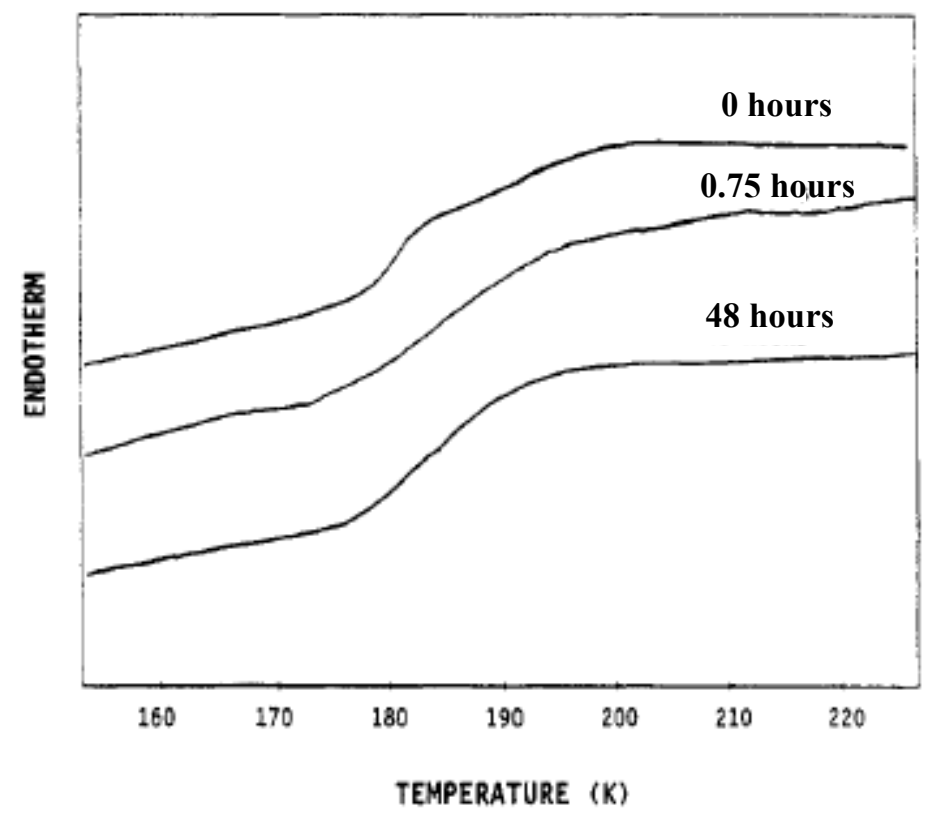

Figure 2-19 Measurements of xenon peaks collapsing vs. differential scanning calorimetric measurements (taken from Ref. 79).

not only was ${ }^{129} \mathrm{Xe}$ NMR more useful than DSC, but also, that it is able to differentiate between polymers with similar $\mathrm{T}_{\mathrm{g}}{ }^{\text {' } \mathrm{s} .}{ }^{79}$ 
The Pulse Field Gradient (PFG) NMR technique was used to determine the xenon self-diffusion coefficients in polymer materials. These reflect the mobility of polymer chains and can be used to estimate domain sizes. The PFG method, improved by Stejskal and Tanner in 1965, is based on applying radiofrequency pulse sequences in order to generate spin echos and measure translational motions ${ }^{80}$ It is based on a Hahn-spin-echo pulse sequence $\left(90^{\circ}-\tau\right.$ $180^{\circ}-\tau-$ acq) during which two field gradient pulses are applied as seen in Figure 2-20. (Average gradient probes today go up to $10 \mathrm{~T} / \mathrm{m} .{ }^{78}$ ) Field gradient pulses of strength $G$ and the duration $\delta$ lie in between the $90^{\circ}$ and $180^{\circ}$ pulse and then between the $180^{\circ}$ and the echo acquisition with a time $\Delta$ separating the two. ${ }^{78,81}$ After a $90^{\circ}$ pulse, the first gradient pulse is applied to de-phase the spins.

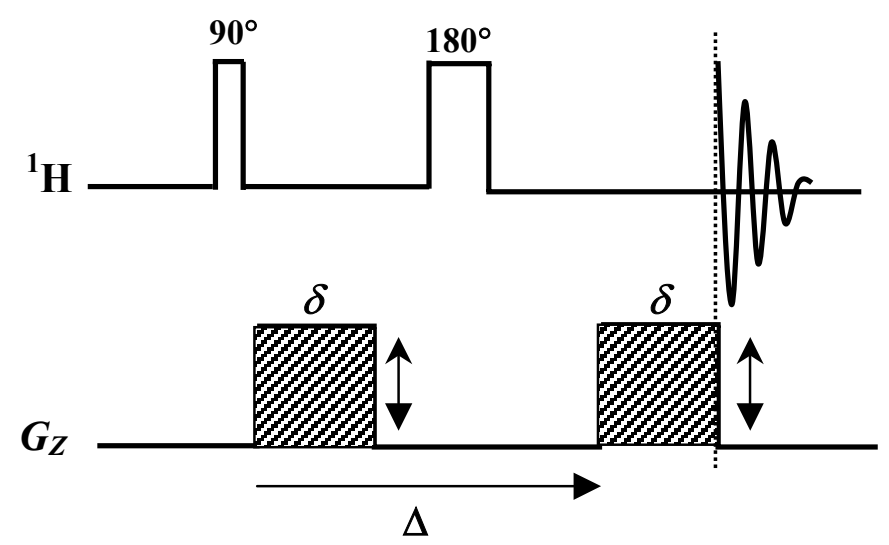

Figure 2-20 Pulse field gradient Hahn spin-echo (taken from Ref. 38).

Position change of spins is monitored with respect to the field gradient direction during time $\Delta$, which will in turn lead to an attenuation of the spin-echo intensity. The $180^{\circ}$ pulse reverses all phase differences and the second $G$ gradient re-phases 
the spins to give a strong spin-echo effect if the spins have not changed position. ${ }^{78}$ The more the molecules carrying the spins move in the time period between the two gradient pulses, the more the echo is attenuated. Also, the stronger the field gradient that is used in the pulse sequence, the lower the diffusion coefficients that can be accurately measured. The following equation can be used to calculate the diffusion coefficient, D:

$$
\ln \left(\frac{A(G)}{A(G=0)}\right)=-\gamma^{2} \delta^{2} D(\Delta-1 / 3 \delta) G^{2}
$$

$A(G)$ is the echo amplitude in the presence of the field gradient, and $\gamma$ is the gyromagnetic ratio of the nuclei being studied. In order to interpret the attenuation of the signal, a plot of $\ln (\mathrm{A}(G) / \mathrm{A}(G=0))$ vs. $-\gamma^{2} \delta^{2}(\Delta-1 / 3 \delta) G^{2}$ where $G$ is being varied, will directly yield D from the slope of the graph. Other factors can

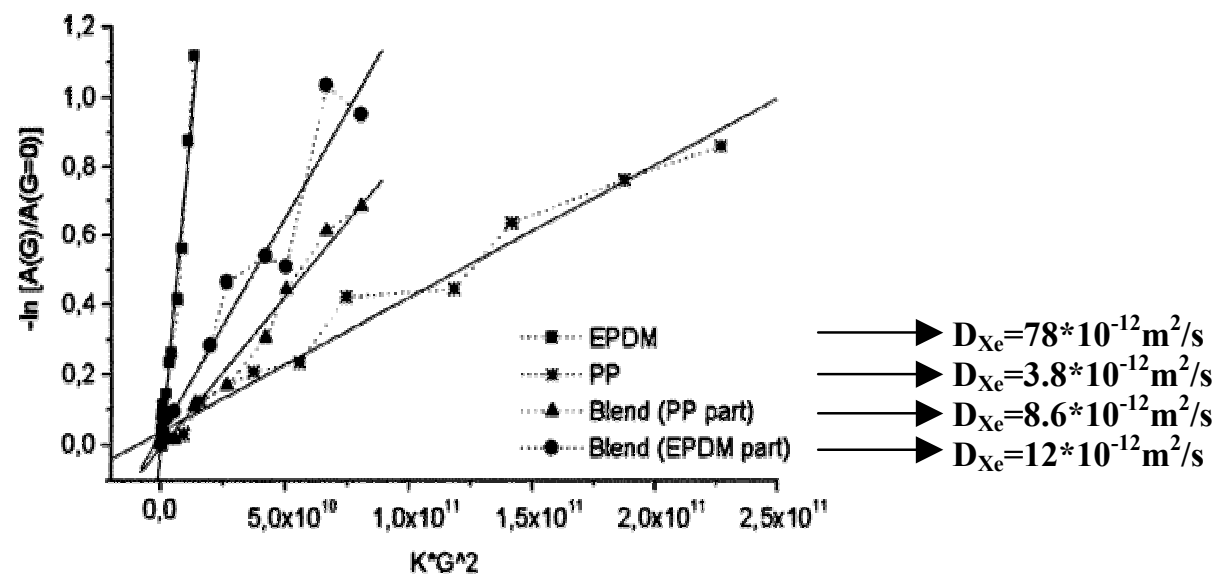

Figure 2-21 Graph of $-\ln (\mathrm{A}(G) / \mathrm{A}(G=0))$ vs. $\gamma^{2} \delta^{2}(\Delta-1 / 3 \delta) G^{2}$ where $\mathrm{K}=\gamma^{2} \delta^{2}(\Delta-1 / 3 \delta)$ directly from which diffusion coefficients can be extracted (indicated on the right) (taken from Ref. 38). 
be varied, like $\delta$ or $\Delta$ to determine the diffusion coefficient but choices in polymer blends are limited due to the short $\mathrm{T}_{2}{ }^{\prime} \mathrm{s}{ }^{78}$ Junker and Veeman compared the xenon diffusion coefficients in the EPDM/PP system, but they actually used a stimulated echo. This pulse sequence circumvents the short $T_{2}$ problems in the Hahn echo sequence by adding a time (in between the two gradients), during which only $T_{1}$ relaxation takes place. As seen from the plot in Figure 2-21, the diffusion coefficient was much faster for pure EPDM as compared with pure PP, and it was intermediate for the blend.

Once the diffusion coefficient is determined, a fairly accurate estimate of the largest domain size in a blend considered to be miscible (meaning it has a single xenon resonance) can be made. The average distance a xenon molecule travels $^{82}$ in time $\tau$ is

$$
X_{r m s}=\sqrt{2 D \tau}
$$

where D stands for the diffusion coefficient. The xenon gas in the polymer system is in the fast exchange limit, meaning that

$$
\tau(\delta \omega)<<1
$$

where $\delta \omega$ is the xenon chemical shift difference between the pure components. Both equations can be combined to establish an upper bound domain size:

$$
X_{\text {domain }}<\sqrt{\frac{2 D}{\delta \omega}}
$$

The above equation will later be used to establish domain sizes for two blends. 
Chapter 3: Dynamic Disorder and Conformer Exchange in the Crystalline Monomer of Polycarbonate

\author{
J. E. Wolak, J. Knutson, J. D. Martin, P. Boyle, Andrew L. Sargent \\ Jeffery L. White* \\ Department of Chemistry, North Carolina State University, \\ Raleigh, NC27695-8204
}

As published in the Journal of Physical Chemistry B, 2003,

Vol.107, p.13293-13299 


\section{3-1 Abstract}

Direct measurement of chemical exchange events in the crystalline polycarbonate monomer $4,4^{\prime}$ - isopropylidenediphenol (Bisphenol A) via $2 \mathrm{D}{ }^{13} \mathrm{C}$ solid-state NMR reveals slow, large-amplitude aromatic ring reorientations. Xray diffraction, however, indicates a static crystalline structure. Experiments with multiple exchange times show that ring flips occur in all of the three unique conformers found in the crystalline unit cell of this compound, but in specific cases, two of the three unique molecules actually switch conformations. Kinetic analysis of the exchange data indicates that the average rate constant $\mathrm{k}_{\mathrm{ex}}=0.01 \mathrm{~s}^{-1}$ for ring flips and conformer interchange at room temperature. DSC and variabletemperature powder diffraction studies indicate a systematic volume expansion that accompanies this motion, but no first-order phase transition. All roomtemperature exchange events may be quenched at $213 \mathrm{~K}$, at least on the timescale (up to several seconds) probed in this work. Simulation of the potential energy surface of BPA molecules reveals that the lowest energy pathway for ring flips (maximum energy of $1.9 \mathrm{kcal} / \mathrm{mole}$ ) involves +110 degree flips of one ring coincident with +70 degree flips of the second. The mechanism of the ring dynamics in the crystalline monomer is unique relative to those previously reported for the polycarbonate, in that the collective motion of both rings in a monomer unit is $180^{\circ}$, whereas single-ring flips of $180^{\circ}$ occur in the polymer.

- Department of Chemistry, East Carolina University, Greenville, NC 


\section{3-2 Introduction}

Bisphenol-A (4,4'-isopropylidenediphenol or BPA) is the crystalline organic monomer of polycarbonate, and in addition, finds application in a variety of epoxy resins. The relationship between dynamics, diffusion, and elution of residual bisphenol A monomer from polycarbonate and its overall chemical toxicity is currently receiving widespread attention in the literature, due to its potential health hazards. ${ }^{83}$ In its pure crystalline form, BPA contains three crystallographically distinct molecules in its unit cell, differing primarily in the relative orientation of the two aromatic rings in each molecule. ${ }^{84-86}$ Intermolecular hydrogen-bonding defines the overall packing of molecules in the crystal, with the characteristic self-association of phenolic compounds leading to the differential molecular configurations. X-ray crystallography provides welldefined atomic coordinates at room temperature (corresponding to the space group $\mathrm{P} 2{ }_{1} / \mathrm{n}$ ), as would be expected for a crystalline solid with a melting point in

excess of $150^{\circ} \mathrm{C}^{84,85}$ Being a highly crystalline solid, we (and others) initially considered solid BPA to be a control compound for rigid-lattice exchange experiments. However, upon detailed examination, we have observed large amplitude local motions associated with aromatic ring reorientation and conformer interchange at temperatures even below ambient.

Aromatic ring dynamics in the solid state have been the subject of recent investigations in the area of glassy polymers, as the molecular-level, largeamplitude motions provide a possible pathway for mechanical energy dissipation. Most notably, Schaefer has demonstrated that $\pi$-flips of the aromatic rings take 
place in glassy polycarbonates. ${ }^{87-89}$ Using dipolar-echo solid-state NMR methods, Schaefer and coworkers identified a broad distribution of $\pi$-flip rates ranging from kilohertz to megahertz, and showed that these molecular motions are relevant to bulk mechanical relaxation in the solid state ${ }^{87}$ Since that time, others have corroborated the presence of aromatic ring $\pi$-flips in several different types of glassy polycarbonates, some small molecule analogues, and other amorphous macromolecules like polystyrene. ${ }^{90-95}$ In this report, we (1) prove that specific subsets of these motions occur in the crystalline monomer, (2) identify the relevant motional amplitudes and timescales, and (3) discern the individual conformers in the unit cell which participate in exchange via these ring motions.

\section{3-3 Experimental}

Polycrystalline 4,4'-isopropylidenediphenol (BPA) was obtained from Aldrich, and used without further modification for the NMR experiments. The melting point of the pure solid is $156^{\circ} \mathrm{C}$, as confirmed using a TA Instruments D100 DSC. Pure-phase $2 \mathrm{D}^{13} \mathrm{C}-{ }^{13} \mathrm{C}$ exchange experiments were acquired using the simple 3-pulse sequence, modified for cross-polarization excitation, and with mixing times synchronized to the rotor period. ${ }^{9,96-98}$ Typically, $128 \mathrm{t}_{1}$ increments were acquired using 48 scans per increment, with subsequent zero-filling to generate a $1 \mathrm{~K} \times 1 \mathrm{~K}$ data matrix prior to Fourier transformation. NMR measurements were acquired on a Bruker DSX instrument, operating at 7.05 T field strength, which corresponds to a ${ }^{13} \mathrm{C}$ resonance frequency of $75 \mathrm{MHz}$. 
Unless otherwise stated, magic-angle spinning (MAS) speeds were 4-5 kHz, and radio-frequency field strengths were $72 \mathrm{kHz}$. Control experiments using $100 \mathrm{kHz}$ continuous wave vs. TPPM decoupling did not produce any improvements in resolution. Similar experiments at 7-8 kHz MAS on an $11.7 \mathrm{~T}$ system also failed to yield any improvements in spectral resolution. ${ }^{1} \mathrm{H}$-mediated ${ }^{13} \mathrm{C}$ spin-diffusion was ruled out as a possible polarization transfer mechanism via comparision of 2D exchange experiments with and without ${ }^{1} \mathrm{H}$-decoupling during acquisition, in which identical cross-peak volumes were obtained. ${ }^{96}$ Cross-peak volumes were integrated for kinetic analysis of the exchange process.

Solid-state ${ }^{1} \mathrm{H}-{ }^{13} \mathrm{C}$ heteronuclear correlation experiments were acquired using Lee-Goldburg irradiation during the evolution period. ${ }^{99}$ While the data are not presented here for brevity, several distinct and well-resolved ${ }^{1} \mathrm{H}$ chemical shifts were detected for the protonated aromatic carbons, with a total chemical shift range exceeding $1 \mathrm{ppm}$. This result is consistent with the expected intramolecular neighboring group shielding contributions to the total shift, and is in agreement with the ${ }^{13} \mathrm{C}$ solid-state data (vide infra).

A solution of 4,4'-isopropyldenediphenol (BPA) and ethanol was prepared and allowed to slowly evaporate for two weeks. Crystals suitable for single crystal X-ray diffraction were obtained. Single-crystal X-ray structures were obtained at $-60^{\circ} \mathrm{C}$, and the results compared to published room-temperature data. ${ }^{86}$ The sample was mounted on the end of a glass fiber using a small amount of silicon grease and transferred to the diffractometer. The sample was maintained at a 
temperature of $-60^{\circ} \mathrm{C}$ using a nitrogen cold stream. All X-ray measurements were made on an Enraf-Nonius CAD4-MACH diffractometer.

Variable-temperature powder x-ray diffraction data were collected using an INEL diffractometer. Samples were placed in $0.5 \mathrm{~mm}$ fused silica capillaries and mounted on a rotating goniometer head. Samples were heated with a homebuilt forced-air furnace with temperature controlled to $\pm 0.5^{\circ}$ using an Omega controller. The powder diffraction data were calibrated to an external silicon standard, and lattice constants were determined by a least squares refinement of $>$ 20 lines.

Full-gradient geometry optimizations were performed in density functional theory with the B3LYP wave function, as implemented in the Gaussian 98 program suite. ${ }^{100}$ The $6-31 \mathrm{G}^{*}$ basis set $^{101}$ was used throughout as a compromise between accuracy and applicability to molecules of this size. Approximate transition state geometries were evaluated with the QST2 algorithm ${ }^{102}$ but were subsequently verified as first-order saddle points with frequency calculations at the B3LYP/6-31G* level of theory. All equilibrium structures were likewise characterized and verified to possess no imaginary frequencies.

\section{3-4 Results and Discussion}

Figure 3-1 shows our first experimental result indicating that aromatic ring reorientation occurs in BPA. These results were obtained quite by accident, in that we were deliberately using the crystalline BPA as a control compound for 
a rigid-lattice exchange experiment. As such, we did not expect to observe any cross-peak intensity connecting the diagonal peaks of the protonated aromatic ring carbons. However, Figure 3-1 clearly shows strong cross-peaks connecting the inequivalent ortho ring carbons of BPA, indicating that some form of magnetization transfer or chemical exchange was occurring. Since cross-peaks were observed only between inequivalent ortho or meta aromatic ring signals, and not all ring carbons, this first result suggested a large amplitude ring flip that interconverted inequivalent ring carbons.

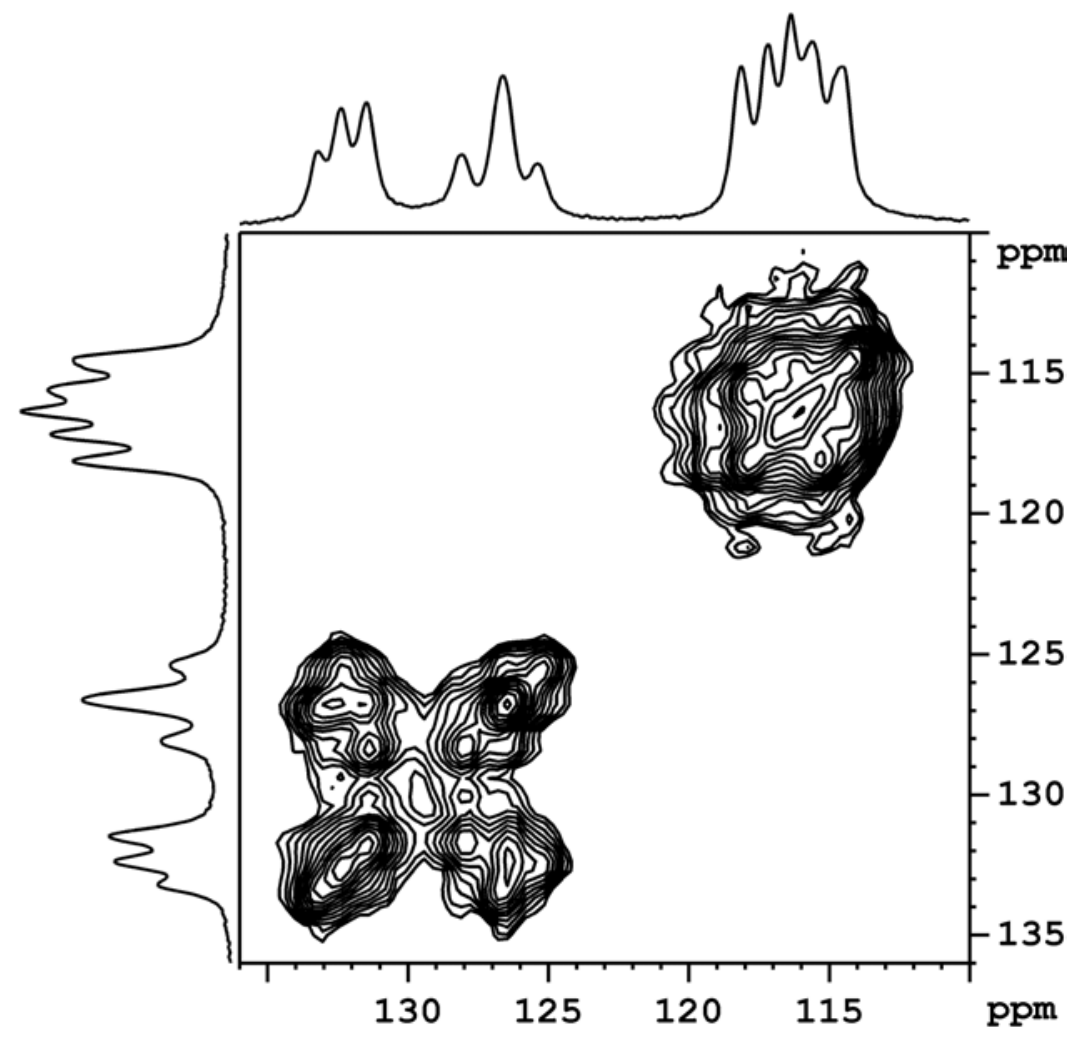

Figure 3-1 2D ${ }^{13} \mathrm{C}-{ }^{13} \mathrm{C}$ exchange plot of the BPA aromatic region at $23{ }^{\circ} \mathrm{C}$ using a 1 second exchange time. 


\section{Variable-Temperature CP/MAS Data}

Figure 3-2 shows the $\mathrm{CP} / \mathrm{MAS}{ }^{13} \mathrm{C}$ spectra of crystalline $\mathrm{BPA}$ as a function of temperature, along with a schematic of the molecular structure indicating the chemical shift assignments. Due to its high crystallinity, BPA linewidths are narrow, and several inequivalent resonances are resolved even at this moderate field strength. No improvements in resolution were obtained by increasing the ${ }^{13} \mathrm{C}$ Larmor frequency to $125 \mathrm{MHz}$ (data not shown). Examination of the aromatic region $(110-160 \mathrm{ppm})$ of the $23^{\circ} \mathrm{C}$ spectrum in $\mathbf{3 - 2 ( b )}$ indicates that at least three unique signals are observed for each ring carbon, consistent with the X-ray assignment of three inequivalent conformations per unit cell. Closer inspection reveals that the ortho ring carbons d and d' (125-135 ppm) have six resolved peaks, and the meta ring carbons e and $\mathbf{e}^{\prime}(110-120 \mathrm{ppm})$ give rise to five well-resolved peaks. In fact, the quaternary aromatic carbon c, which has three well-resolved signals, shows evidence for additional inequivalent sites in the asymmetric lineshape for the intense 145 ppm signal.

Comparison of the $23^{\circ} \mathrm{C}$ result in $3-2(b)$ with spectra obtained at $-60^{\circ} \mathrm{C}$ (32(a)) and $80^{\circ} \mathrm{C}(3-2(c))$ reveal large changes in the peak intensity and resolution for the protonated aromatic ring carbons in the 110-140 ppm region. Specifically, the number of resolved spectral features in this region decreases with increasing temperature. Inspection of the meta carbon region (110-120 ppm) in 3-2(a) shows at least seven peaks, relative to five in 3-2(b), and the fourth component of ring carbon $\mathbf{c}$ is now apparent relative to the room temperature result. Additional broadening of the $\mathbf{d} / \mathbf{d}^{\prime}$ carbon region at $-60^{\circ} \mathrm{C}$, consistent with the introduction of 
additional peaks with small chemical shift separations, is also observed. Taken together, these results indicate that a small degree of motional averaging of inequivalent ring carbon positions takes place as the acquisition temperature is increased from -60 to $23^{\circ} \mathrm{C}$. Consideration of the differential packing effects and relative ring plane conformations in each molecule can be used to predict the maximum number of spectral lines observed for each type of ring carbon, in the limit of no motion and infinite spectral resolution. In the low-temperature result of Figure 3-2(a), which was obtained using $100 \mathrm{kHz}{ }^{1} \mathrm{H}$-decoupling, the fact that more than six peaks are observed for the meta carbons e/e' and more than three peaks are observed for the quarternary ring carbon $\mathbf{c}$ indicate that either there is inequivalence between the aromatic ring carbons of each ring in a single molecule, or that more than 3 unique molecular conformations exist in the crystalline unit cell. In the limit of 3 conformers, and complete inequivalence between the ring carbons of each ring in one molecule, then 12 peaks would be expected for the ortho carbons, 12 peaks for the meta carbons, and 6 peaks for each of the quaternary aromatic carbons $\mathbf{c}$ and $\mathbf{f}$.

Comparison of 3-2(b) with spectra obtained at $80^{\circ} \mathrm{C}(\mathbf{3 - 2 ( c )})$ and $100^{\circ} \mathrm{C}$ (3-2(d)) show large changes in the spectral patterns for the ortho and meta ring carbons only. Coalescence and ultimately, collapse, of each group of inequivalent signals is observed at these elevated temperatures for these off-axis ring carbons. Perturbations in the intensity and resolution of ring carbons $\mathbf{c}$ and $\mathbf{f}$ are observed, as well as for the inequivalent methyl carbons $\mathbf{a}$ and $\mathbf{a}$, but the effects are small compared to the "fast-exchange" response for protonated ring carbons. The 
differential averaging of signals indicates a mode of large-amplitude aromatic ring reorientation about the $\mathrm{C}_{2}$ axis. Based on the chemical shift separation of the ortho signal manifold in the static limit of Figure 3-2(a) (8 ppm), the minimum exchange rate at the coalescence point observed in $\mathbf{3 - 2 ( d )}$ is $600 \mathrm{~s}^{-1}$.

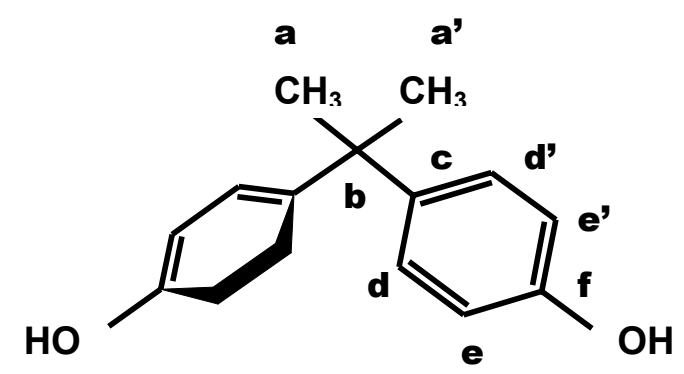

(a)
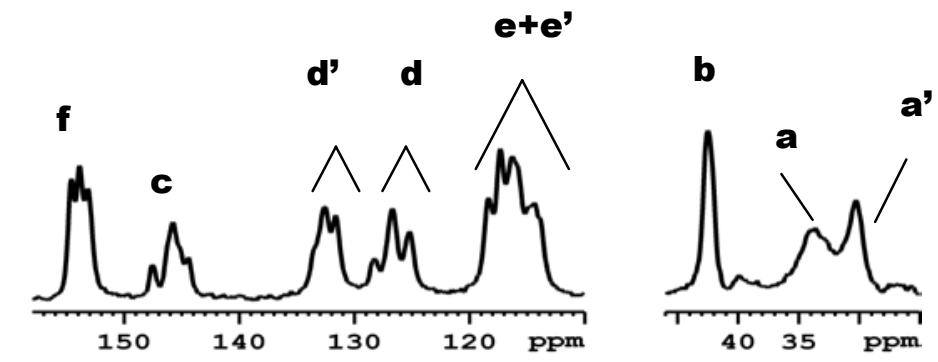

(b)
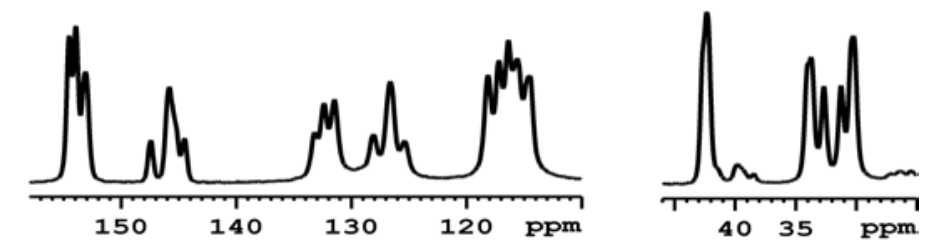

(c)
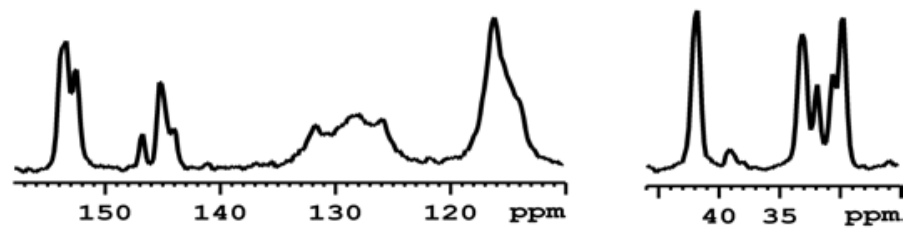

(d)
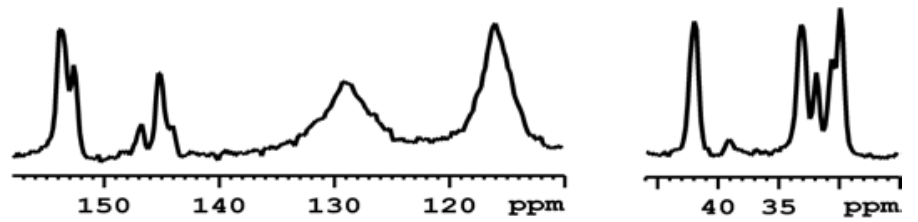

Figure 3-2 $\mathrm{CP} / \mathrm{MAS}$ spectra of $\mathrm{BPA}$ at (a) $-60^{\circ} \mathrm{C}$, (b) $23^{\circ} \mathrm{C}$, (c) $80^{\circ} \mathrm{C}$, and (d) $100^{\circ} \mathrm{C}$. Assignments are indicated on the BPA structure. 


\section{Two-Dimensional ${ }^{13} \mathrm{C}-{ }^{13} \mathrm{C}$ Exchange Data}

The data in Figures 3-1 and 3-2 indicate that ring dynamics do occur in the crystalline BPA molecule as a function of temperature. However, it is not clear from these data what is the (a) amplitude of the motion vs. temperature; (b) relevance of the motion to conformational inequivalence; and (c) sequence of motions, i.e., parallel or concerted vs. sequential. Additional 2D NMR experiments as a function of mixing time and temperature have been completed to address these questions. Figure 3-3 presents the results of $2 \mathrm{D}{ }^{13} \mathrm{C}$ exchange experiments at $23^{\circ} \mathrm{C}$ and $-60^{\circ} \mathrm{C}$. Figures 3-3(a) and 3-3(b) present the $23^{\circ} \mathrm{C}$ results for mixing times of $100 \mathrm{~ms}$ and $2 \mathrm{~s}$, respectively, while the $100-\mathrm{ms}$ result at $-60^{\circ} \mathrm{C}$ is shown in 3-3(c). Figure 3-3(a) shows that even at relatively short millisecond-timescales, ring reorientation that acts to exchange the inequivalent ortho and meta ring carbons among themselves takes place at room temperature. The cross-peak pattern is almost fully developed in 3-3(a), as may be observed by comparison with the 2-s exchange time result in 3-3(b). While the individual cross-peaks between the inequivalent ortho ring carbons are well-resolved from the diagonal, ring reorientation manifests itself in the meta carbons as broadening of the diagonal. In some cases, individual cross-peaks between the e/e' carbons are apparent. This exchange is quenched on the $100-\mathrm{ms}$ timescale at $-60^{\circ} \mathrm{C}(3-$ 3(c)), and additional experiments with a 2 -s exchange time at $-60^{\circ} \mathrm{C}$ produced a similar spectrum with no cross-peaks (not shown).

An expanded view of the aromatic ring ortho carbon region is shown in Figure 3-4 for an exchange time of $500 \mathrm{~ms}$ at $23^{\circ} \mathrm{C}$. Equilibrium exchange is 

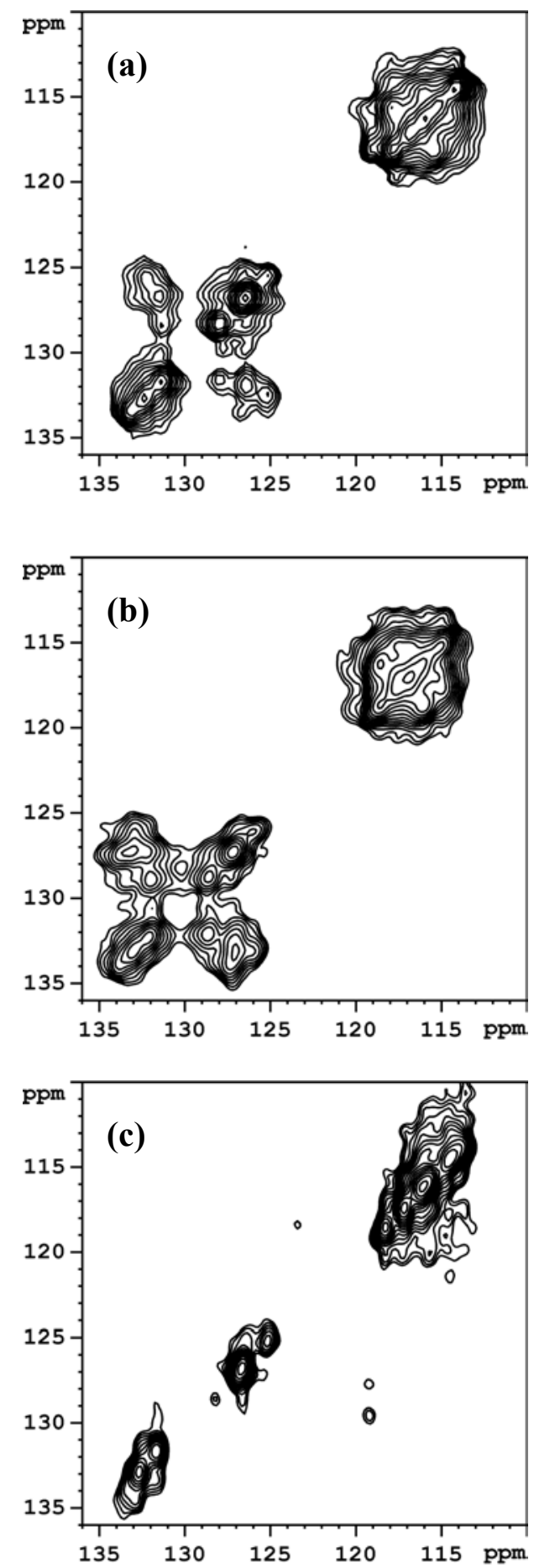

Figure 3-3 2D exchange spectra of the aromatic BPA region at (a) $23^{\circ} \mathrm{C}, 0.1 \mathrm{~s}$ exchange; (b) $23^{\circ} \mathrm{C}$, $2 \mathrm{~s}$ exchange; (c) $-60^{\circ} \mathrm{C}, 1 \mathrm{~s}$ exchange.

essentially complete at this mixing time, due to equal diagonal and cross-peak intensities. New features are not observed at longer exchange times. Indeed, the 
individual cross-peaks look very similar to the 100-ms result in 3-3(a), albeit with much greater intensity relative to the diagonal. Specifically, four distinct crosspeaks may be observed connecting the $\mathbf{d} / \mathbf{d}$ ' ortho carbons. The fact that more than three cross-peaks appear indicates that there are additional conformer dynamics other than just simple exchange of an ortho carbon on one ring (d) to its inequivalent neighbor (d'). The diagonal peaks at 126.2 and 131.3 both show two correlations, while the remaining four peaks only exhibit single cross-peaks. The fact that less than nine cross-peaks appear proves that complete interchange between all inequivalent ortho ring positions in all conformers does not occur. Figure 3-4 proves that in addition to simple exchange of ortho carbons $\mathbf{d}$ and $\mathbf{d}$ ' within a single conformation, the large amplitude aromatic ring reorientation facilitates exchange between two of the three different conformers, as shown by the isotropic peaks at 126.2 and 131.3 that exhibit multiple correlations. For example, if one examines the 131.3 ppm peak, two possible events exist. First, the ring motion can exchange an ortho with its ortho' position, given by the correlation with the $128 \mathrm{ppm}$ peak, or, conformer exchange can take place, as indicated by the correlation with the $126.2 \mathrm{ppm}$ peak. A similar situation exists for the $126.2 \mathrm{ppm}$ peak, in that simple ring exchange gives rise to a correlation with the $133 \mathrm{ppm}$ peak. In both cases, the exchange pathway connecting the two inequivalent conformers is one-way, i.e., the $128 \mathrm{ppm}$ peak does not correlate with the $133 \mathrm{ppm}$ peak. The conformer represented by the $\mathbf{d} / \mathbf{d}$ ' isotropic peaks at 125 and 132.3 , respectively, undergoes only ring reorientation. 

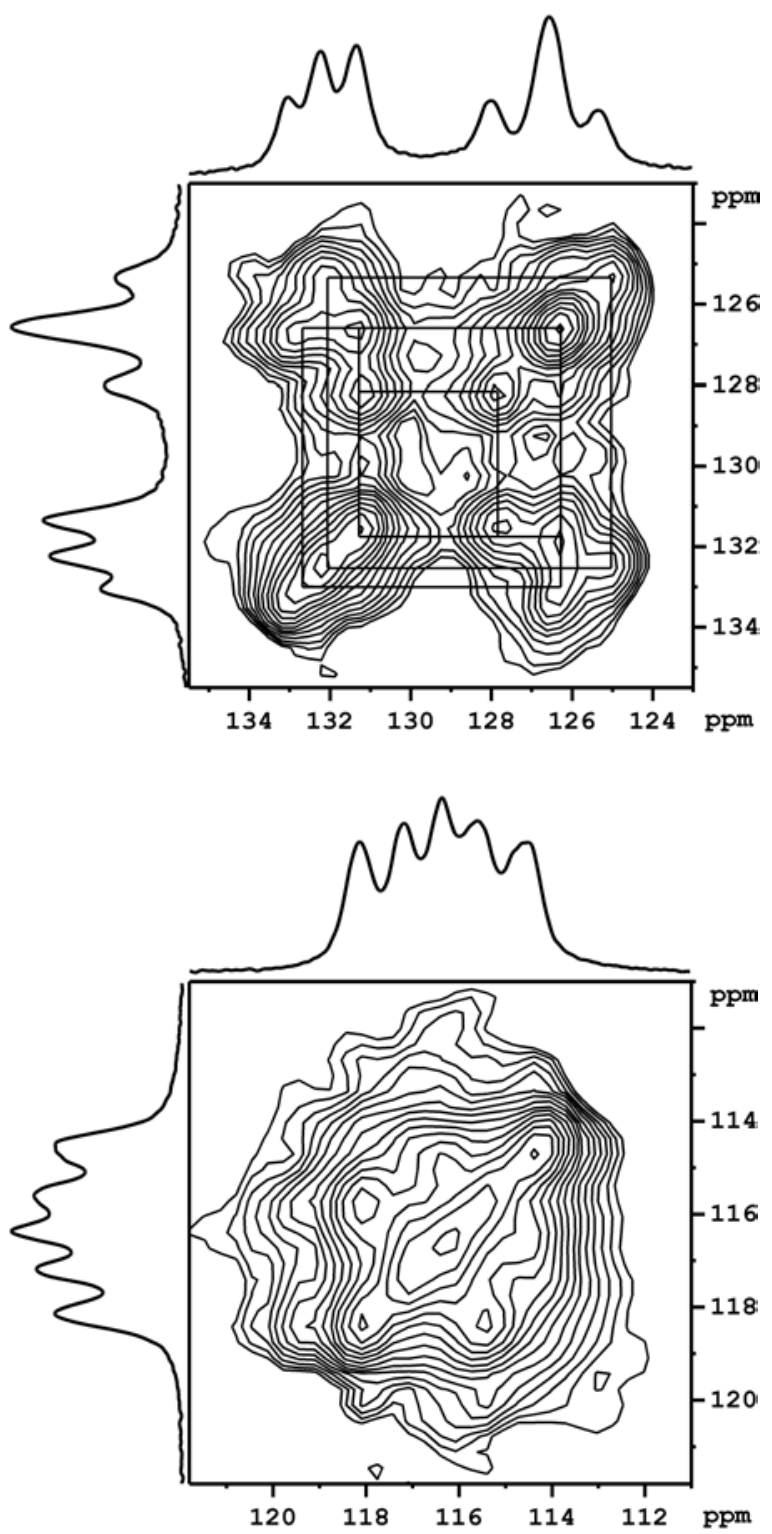

Figure 3-4 Expanded view of the (a) ortho and (b) meta ring carbons of BPA at $23^{\circ} \mathrm{C}$ using a $0.5 \mathrm{~s}$ exchange time. In (a), straight lines are used to indicate the exchange pathways. From these, one observes that only two conformers share multiple correlations.

\section{Exchange Kinetics}

The room-temperature kinetic analysis of BPA dynamics is shown in Figure 3-5, in which the total cross-peak volumes have been plotted vs. the mixing time. Since the integrated cross-peak region necessarily contains intensity 
arising from both ring flips and conformer exchange events, averaged kinetic rate information is obtained. However, since ring flips are the mechanism by which two of the three conformers exchange with one another, this is a physically relevant average. The fact that the $2 \mathrm{D}$ exchange data implies two types of exchange events (simple ring flips and ring flip/conformer interchange) is consistent with the fits to the data shown in Figure 3-5. The dashed singleexponential line does not fit the initial rate, but does fit the long-time region. A bi-exponential fit (solid line) accurately models the initial exchange rate (see inset), as well as longer time events, but does not reproduce the intermediate range data well. Exchange rate constants for the bi-exponential fit to the equation $\mathrm{y}=\mathrm{a}\left(1-\exp \left(-\mathbf{k}_{\mathbf{1}} \mathrm{x}\right)\right)+\mathrm{b}\left(1-\exp \left(\mathbf{k}_{\mathbf{2}} \mathrm{x}\right)\right)$ yielded $\mathbf{k}_{\mathbf{1}}=0.06 \mathrm{~s}^{-1}$ and $\mathbf{k}_{\mathbf{2}}=0.006 \mathrm{~s}^{-1}$. Obviously, higher-order exponentials, or stretched exponentials, could fit all regions of the data. However, the bi-exponential model is consistent with the physical events revealed in the $1 \mathrm{D}$ and $2 \mathrm{D}$ exchange NMR data.

\section{Single-Crystal and Powder X-Ray Data}

The crystalline unit cell of BPA is shown in Figure 3-6, with color identification of the three inequivalent conformers. The unit cell contains twelve molecules; four each of the three unique conformers. In particular, it appears that the blue and green conformers are more closely equivalent relative to the yellow molecule, as may be observed by inspection of the packing arrangement of each with its neighbors. By inspection of the packing arrangement, it is possible to envision a pairwise molecular flip by which the blue and green conformers relate via a pseudo four-fold axis. (This would be expected to occur at higher 
temperatures than those considered in this study). Other than the expected increase in unit cell volume, the crystal coordinates for single-crystals at $-60^{\circ} \mathrm{C}$ and $23^{\circ} \mathrm{C}$ are equivalent. This confirms that on the timescale of the $\mathrm{x}$-ray

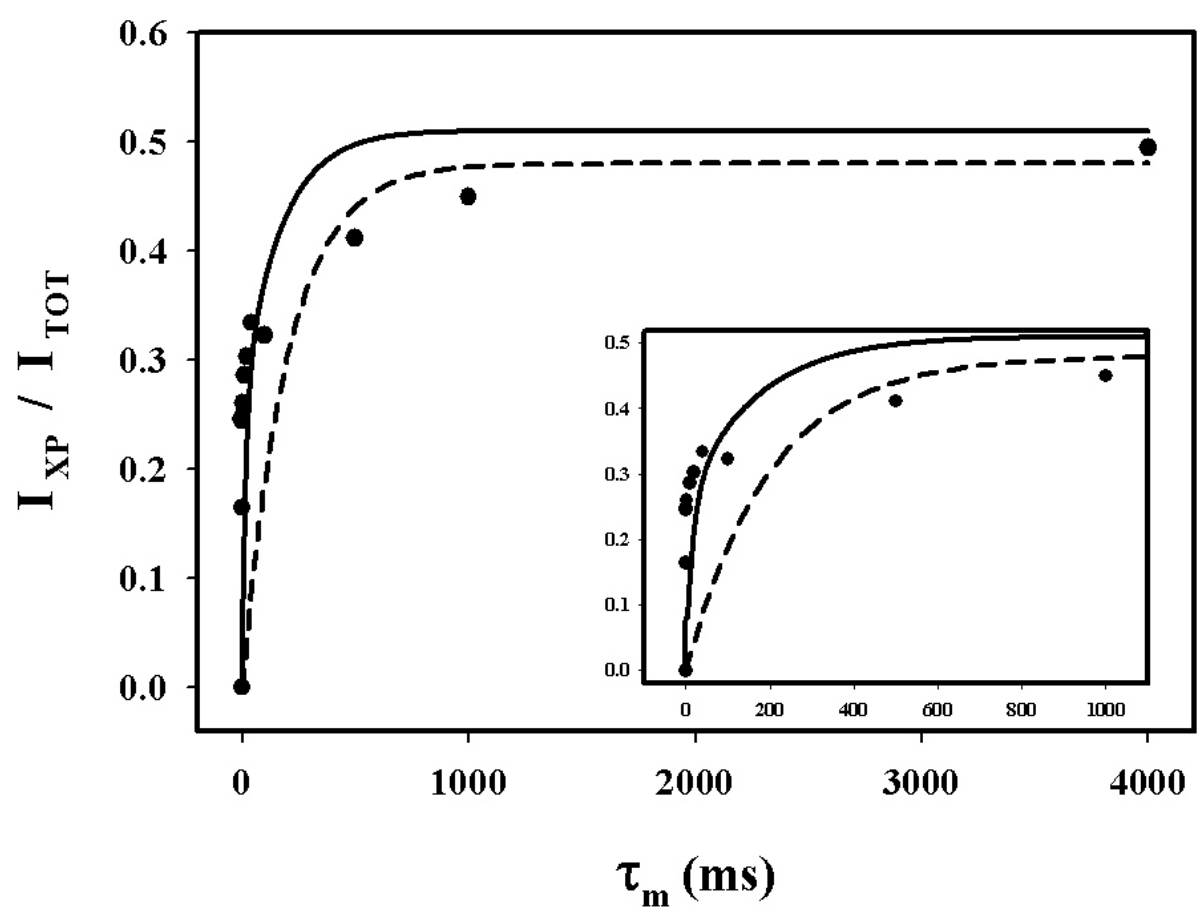

Figure 3-5 Kinetic plot of the exchange cross peak volume fraction as a function of exchange time $\tau_{\text {mix }}$ for single (-----) and double ( - ) exponential fits. The equations used in the single and double exponential fits are $y=a\left(1-\exp \left(-k_{1} x\right)\right)$, and $y=a\left(1-\exp \left(-k_{1} x\right)\right)+b\left(1-\exp \left(k_{2} x\right)\right)$, respectively.

experiment (several seconds), the lattice is static. Variable temperature powder diffraction data, as well as DSC, did not reveal any first-order phase transitions (ambient to $100^{\circ} \mathrm{C}$ ). However, as expected, thermally induced unit cell expansion was observed from the X-ray powder data as described in Table 3-1.

\section{Simulation of Ring Motions}

While the 2D solid-state NMR data unequivocally prove that some type of large amplitude ring reorientation exists in BPA, and that the result of that motion 
is to interchange inequivalent ortho and meta positions on adjacent rings of the same molecule, it does not reveal the amplitude or relative direction of ring reorientation. If BPA molecules with specific ${ }^{13} \mathrm{C}$ labels at one ortho or meta position were available, then static 2D CSA exchange experiments could identify the motional amplitude directly. ${ }^{101}$ However, in the absence of these expensive models, we have used DFT calculations to evaluate various types of ring motion and construct the corresponding potential energy surfaces for these motions in BPA.

The X-ray data (Figure 3-6) shows that within a given molecule of BPA, each of the $\mathrm{C}-\mathrm{C}_{\mathrm{Me}}$ bond vectors lie in the same plane as a single aromatic ring. ${ }^{62}$ Whether the para-hydroxyl groups on the rings orient themselves syn or anti to the corresponding methyl vectors gives rise to three possible conformational isomers, as confirmed by the gas-phase DFT calculations. Although the syn-syn isomer is calculated to be the lowest in energy, all three are within a few hundredths of a kcal per mole of each other, as shown in Figure 3-7. Various types of ring motion will satisfy the requirements of the exchange data in Figures 3-1--3-4. DFT calculations revealed that the simple $180^{\circ}$ rotation of a given phenyl ring can be broken down into smaller amplitude rotations that effect the same result. As shown in Scheme 1, either a $110^{\circ} \mathrm{cw}$ rotation or a $70^{\circ} \mathrm{ccw}$ rotation of the highlighted ring will effect the desired exchange. While both rings in BPA are free to utilize either movement, results from Figure 3-7 show that the lowest energy movements correspond to both rings moving in the same direction when viewing the molecule along the molecular long axis. This means that one 


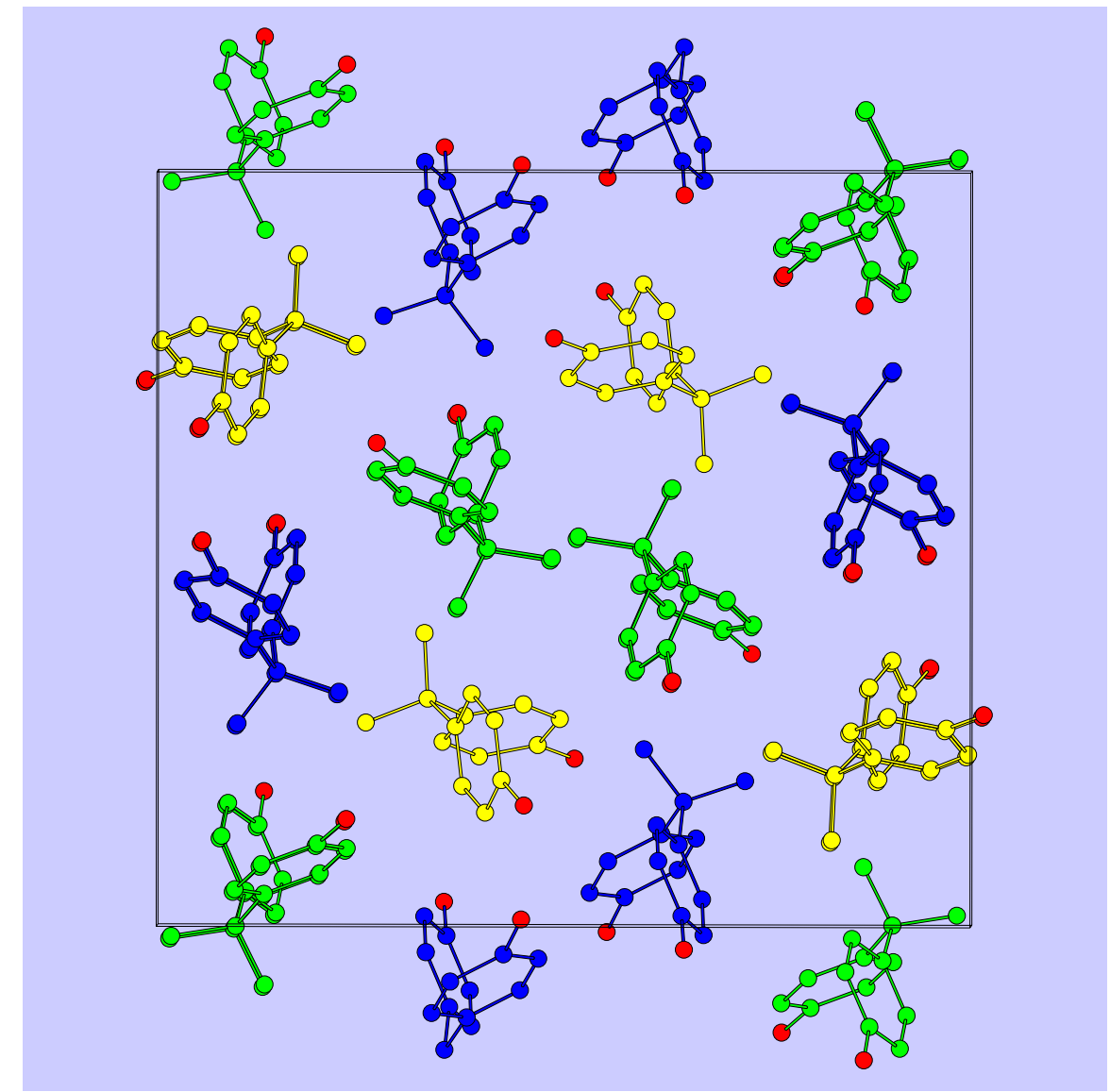

Figure 3-6 Unit cell structure of BPA, viewed along the a axis, derived from the $23^{\circ} \mathrm{C}$ single crystal $\mathrm{x}$-ray data. The three inequivalent molecular conformations are denoted by color.

Table 3-1 X-ray powder data

\begin{tabular}{|c|c|c|}
\hline$\frac{\text { Temperature }}{(\mathbf{K})}$ & $\frac{\text { Unit cell volume }\left(\mathbf{n m}^{3}\right)}{\text { (single crystal) }}$ & $\frac{\text { Unit cell volume }\left(\mathbf{n m}^{3}\right)}{(\text { powder) }}$ \\
\hline 213 & 3.738 & \\
\hline 293 & $3.774 *$ & 3.728 \\
\hline 303 & & 3.769 \\
\hline 313 & & 3.778 \\
\hline 323 & & 3.790 \\
\hline 333 & & $\mathbf{3 . 7 9 3}$ \\
\hline 343 & & $\mathbf{3 . 8 2 0}$ \\
\hline 353 & & $\mathbf{3 . 8 2 4}$ \\
\hline
\end{tabular}

*obtained from Ref. 85 
of the phenyl rings will move by $110^{\circ}$ while the other moves by $70^{\circ}$, with barrier heights in the range of 1.69 to $1.88 \mathrm{kcal} / \mathrm{mol}$. Movement of the rings in opposite directions, both through a $70^{\circ}$ arc, yields a higher energy barrier of $3.25 \mathrm{kcal} / \mathrm{mol}$. Some limitations of the theory must be mentioned. First, these results are from gas-phase/isolated molecule calculations. Unfortunately, calculations involving the entire unit cell structure and the associated intermolecular energies are not feasible given the available computational resources. Further, the three conformers identified in the simulations should not necessarily be associated with prediction of the unit cell conformer population, since it is unclear to what extent the hydrogen-bonding network is perturbed when a ring reorients. In other words, the simulations in Figure 3-7 allowed the phenolic O-H bond vector to move, while this may or may not occur in the crystalline solid state. (Preliminary infrared spectroscopy results indicate that the hydrogen bonding network is also dynamic at room temperature and below. ${ }^{103}$ ) The three conformers that are identified in the x-ray results differ in slight perturbations of the orientation of the aromatic ring planes relative to the $\mathrm{CH}_{3}$ bond vector direction, within a molecule. While packing effects may induce these perturbations, which are not described by

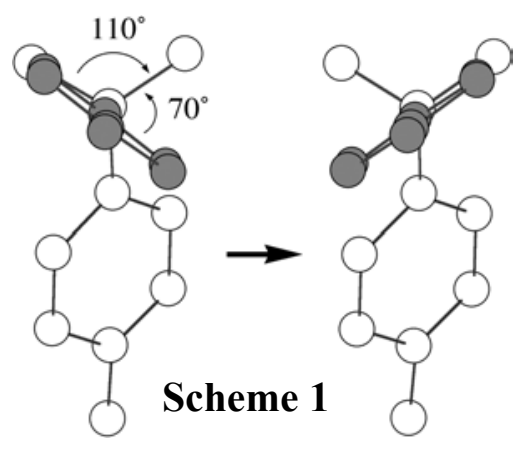


the simulations, the intramolecular barriers to ring reorientation should rank the possible motions accurately in terms of the ring rotation amplitude. Scheme 1 illustrates one type of ring motion that contributes to the overall BPA dynamics shown in Figure 3-7.

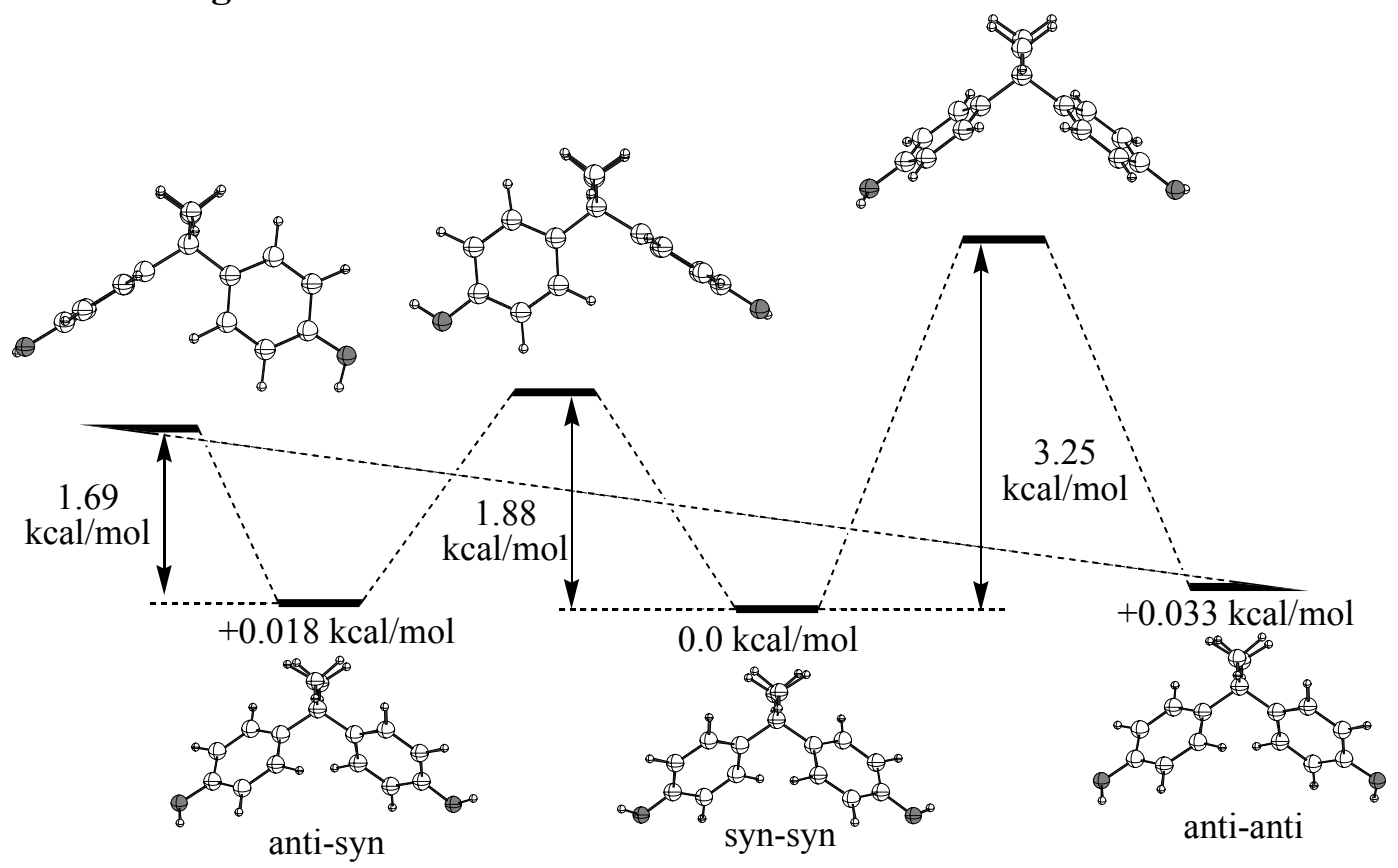

Figure 3-7 Potential energy diagram for the gas-phase simulation of ring dynamics in isolated BPA molecules, evaluated at the B3LYP/6-31G* level of theory. Energies of the three low energy equilibrium isomers are shown relative to the lowest energy isomer. Energies of approximate transition state structures, determined with QST2 methods at the same level of theory, are shown as barrier heights.

\section{3-5 Conclusions}

Detailed solid-state NMR experiments have unequivocally revealed the presence of large amplitude, low frequency ring reorientations in the crystalline BPA polycarbonate monomer in a manner consistent with recently published reports in other crystalline organic solids. ${ }^{104,105}$ In addition to the exchange of magnetically inequivalent ring carbons, this motion allows two of the three inequivalent molecular conformers in the crystalline unit cell to interchange with 
one another. Density functional theory calculations revealed that the lowest energy pathway for ring dynamics, which satisfied the experimental data, involved concerted rotation of each ring in a molecule by $71^{\circ}$ and $110^{\circ}$, respectively, in the same rotation direction. While other rotation modes were found, e.g., opposite rotation of each ring by $+71^{\circ}$ and $-71^{\circ}$, or $180^{\circ}$ ring flips, the activation barrier was significantly larger than the $1.8 \mathrm{kcal} /$ mole observed for the

preferred motion. Kinetic analysis of the ring dynamics, using the 2D exchange results, was consistent with two disparate exchange phenomena, i.e. equivalent ring flips within a single conformer and ring flips which lead to conformer interchange. Our results indicate that discrete subsets of the overall ring motion previously reported for polycarbonates do take place in the crystalline monomer, and act to provide a pathway for conformer exchange between two of the three inequivalent molecular conformations.

\section{3-6 Supporting Information}

Coordinates from the $-60^{\circ} \mathrm{C}$ single-crystal $\mathrm{x}$-ray experiments are included as supporting information in CIF ascii file format in the Appendix A-3. 


\title{
Chapter 4: Polyolefin Miscibility: Solid-State NMR Investigation of Phase Behavior in Saturated Hydrocarbon Blends
}

\author{
Justyna Wolak, Xin Jia, Hanna Gracz, Edward O. Stejskal, and Jeffery L. White* \\ Department of Chemistry, Campus Box 8204 \\ North Carolina State University, Raleigh, NC 27695-8204 \\ Marcin Wachowicz and Stefan Jurga \\ Institute of Physics, Adam Mickiewicz University \\ Poznan, Poland 61-712
}

As published in Macromolecules, 2003, Vol.36, p.4844-4850 


\section{4-1 Abstract}

Chain-level mixing in polyolefins is investigated for blends of polyisobutylene (PIB) and polyethylene-co-1-butene (PEB). Previous reports suggest that PIB exhibits unusual mixing behavior in certain saturated blends relative to other polyolefins, even though it is immiscible with most. Variabletemperature ${ }^{1} \mathrm{H},{ }^{2} \mathrm{H},{ }^{13} \mathrm{C}$, and ${ }^{129} \mathrm{Xe}$ NMR experiments are used to characterize local PIB chain dynamics in blends with PEB in which the concentration of 1butene comonomer units is 23 or 66 weight $\%$. Results from $1 \mathrm{D}$ and $2 \mathrm{D}$ solidstate ${ }^{13} \mathrm{C}$ exchange experiments, ${ }^{1} \mathrm{H}$ relaxation measurements, and ${ }^{2} \mathrm{H}$ lineshape analysis indicate that local conformational dynamics of the $\mathrm{PIB} \mathrm{CH}_{2}$ group in the polymer backbone increase significantly in blends with PEB copolymers containing 66 weight \% butene comonomer (PEB-66). Even though the PEB-66 is a higher $T_{g}$ polymer than PIB, PIB exhibits a lower effective $T_{g}$ when the blend is formed, relative to its pure state. Similar perturbations are not observed in PIB/PEB-23 blend, indicating that this blend is not miscible at the chain level. These results are directly relevant to the length scale of glass transitions in polyolefins, indicating that local interchain packing plays an important role in the conformational dynamics that occur within a chain, and are suggestive of local configurational entropy contributions to mixing. Although ${ }^{1} \mathrm{H}$ spin-diffusion experiments could not reveal quantitative length scales of mixing in the these blends due to insufficient contrast between the constituents, ${ }^{129} \mathrm{Xe}$ NMR experiments showed that the PIB/PEB-66 blend was homogeneous on a $50-\mathrm{nm}$ length scale. In agreement with the heterogeneous morphology indicated by the 
dynamic NMR experiments, ${ }^{129}$ Xe NMR showed two resolved peaks for the PIB/PEB-23 blend, indicative of phase separation on a 50-nm length scale. The compilation of all the data, most of which was obtained at natural abundance, indicates that the PIB/PEB-66 blend exhibits intimate chain-level mixing on a length scale much shorter than $\mathrm{R}_{\mathrm{g}}(\mathrm{ca} .8 \mathrm{~nm})$. More importantly, the data show that reduced chain packing constraints occur in the miscible blend, and suggest that local entropic contributions are the driving force for miscibility.

\section{4-2 Introduction}

Polyolefins and their blends are a commercially important class of materials, and new polymer architectures now accessible via metallocene catalysis insure that a growing number of novel blend compositions are forthcoming. ${ }^{5-7}$ These synthetic advances have revitalized the structure-property investigations of polyolefins and their copolymers, by virtue of the improved control of traditional comonomer incorporation and the increased flexibility in copolymerizing bulky monomers. ${ }^{5}$ The relative number of possible polyolefin blends now routinely accessible has clearly increased, and the prospects of "engineering" phase behavior in polymer/copolymer blends by rational comonomer incorporation is a realistic target for lighter weight, higher strength, and lower cost materials. However, questions remain as to how mutual solubility in polyolefin blends depends on polymeric microstructure, tacticity, comonomer incorporation, and chain dynamics, and further, how each of these architectural and physical 
characteristics contributes to the interplay between enthalpy and entropy that ultimately determines bulk phase behavior. Following the seminal work in the area of polyolefin miscibility by the Graessley, Lohse, and Bates groups, ${ }^{10-12,14-17,}$ 27-29 we have pursued magnetic resonance investigations of microscopic miscibility and dynamics in polyolefin blends using solid-state NMR methods. ${ }^{33-}$ ${ }^{35} \mathrm{We}$ are particularly interested in polyolefin blends that contain polyisobutylene (PIB) as one component, not only due to our long-standing interest in this polymer, ${ }^{36,48}$ but also due to reports that it exhibits negative $\chi$ (negative enthalpy) behavior with certain other polyolefins. ${ }^{11}$ Such behavior is unusual for blends of completely saturated polymers, as no obvious chemical interactions exist that might drive mixing. In particular, we have shown that PIB exhibits chain-level mixing with head-to-head polypropylene (hhPP), ${ }^{34}$ while others have reported that it phase separates in blends with normal atactic head-to-tail PP. ${ }^{15}$ Given the unique chain-packing properties of PIB (which give rise to its barrier properties), we feel that this apparent regio-selectivity might arise from differential interchain packing geometries. Based on previous reports by Graessley, Lohse, and coworkers, ${ }^{10-12,14-17,27-29}$ we are studying blends of PIB with poly(ethylene-co-1butene) copolymers (PEB), in which the relative comonomer concentration in the PEB is either 23 or $66 \mathrm{wt} \%$ (13 and 49 mole\%, respectively). Our goals in this work are to interrogate length scales of mixing in the absence of extensive deuterium labeling, as well as determine the relationship between local backbone conformational barriers and miscibility. 


\section{4-3 Experimental}

Commercial polyisobutylene $\left(\mathrm{M}_{\mathrm{w}}=1,000,000\right)$ was obtained from ExxonMobil Chemical. The PEB-66 $\left(\mathrm{M}_{\mathrm{w}}=114,000\right)$ is the same polymer previously referenced as HPB66 by Graessley and coworkers, and is a monodisperse ethylene-butene copolymer obtained by anionic polymerization of butadiene, followed by hydrogenation. The degree to which the diene polymerizes 1,2 vs. 1,4 addition determines the butene and ethylene concentrations, respectively, as has been extensively discussed in previous papers. ${ }^{11}$ The PEB-23 sample is a commerical ethylene-butene copolymer made via metallocene polymerization $\left(\mathrm{M}_{\mathrm{w}}=79,000\right)$, and sold as Exact 4041 by ExxonMobil. $50: 50 \mathrm{wt} \%$ blends were prepared from dissolution in toluene for 24-48 hours, followed by solvent evaporation, and then vacuum drying to $10^{-2}$ torr for 4 days or longer. DSC measurements indicated that PIB, PEB-66, and PEB23 polymers had glass transition temperatures of $-68^{\circ} \mathrm{C},-48^{\circ} \mathrm{C}$, and $-45^{\circ} \mathrm{C}$, respectively.

${ }^{13} \mathrm{C}$ and ${ }^{1} \mathrm{H}$ NMR measurements on bulk polymers/blends were acquired on a Bruker DSX-300 instrument, operating at a field strength of 7.05 T. Unless otherwise stated, magic-angle spinning (MAS) speeds were 3-5 kHz, and radiofrequency field strengths were $73 \mathrm{kHz}$. Cross-polarization contact times were $300 \mu \mathrm{s}$, chosen to enhance the relative intensity of the PIB backbone $\mathrm{CH}_{2}$ group, which has a short $T_{1 \rho \mathrm{H}}$ at all temperatures used in this investigation. Pure-phase 2D ${ }^{13} \mathrm{C}-{ }^{13} \mathrm{C}$ exchange experiments were acquired using the simple 3-pulse sequence, modified for cross-polarization excitation, and with mixing times 
synchronized with the rotor period..$^{9,96}$ A sixteen-step phase-cycling routine with TPPI was used to achieve absorption line-shapes in each dimension. Typically, 64-128 $t_{1}$ increments were acquired using 64,96 , or 128 scans per increment, with subsequent zero-filling to generate a $1 \mathrm{~K} \times 1 \mathrm{~K}$ data matrix prior to Fourier transformation. ${ }^{1} \mathrm{H}$ spin-lattice and rotating-frame spin-lattice measurements were done using standard methods in which the carbons were observed as a function of the ${ }^{1} \mathrm{H}$ saturation recovery and spin-locking times prior to cross-polarization, respectively.

${ }^{129} \mathrm{Xe}$ NMR experiments were performed on a Bruker AVANCE $500 \mathrm{MHz}$ spectrometer with an Oxford narrow bore magnet. A 10-mm ID 1H/BB probe (BB500-10EB, Nalorac Cryogenic Corp.) was used for all measurements. All spectra were acquired at $298 \mathrm{~K}$. The line from free xenon gas was used as the internal standard $(0 \mathrm{ppm})$. The instrumental parameters for acquisition of the onedimensional, single-resonance ${ }^{129} \mathrm{Xe}$ NMR spectra are as follows: spectrometer frequency $=138.365 \mathrm{MHz}$; spectral width $=83333 \mathrm{~Hz}$ (equivalent to $602 \mathrm{ppm}$ ); number of data points $=16 \mathrm{~K}$; relaxation delay $=2 \mathrm{~s}$; acquisition time $=0.098 \mathrm{~s}$; pulse width $=16 \mu$ s (tip angle $\left.=60^{\circ}\right)$; number of transients $>1 \mathrm{~K}$. The silhouette of the sample container duplicates that of the ordinary $10-\mathrm{mm}$ NMR tube installed in the sample spinner. In place of the glass tube, there is an Ultem tube $(\mathrm{OD}=10$ $\mathrm{mm}, \mathrm{ID}=6.35 \mathrm{~mm})$. Ultem does not absorb a significant amount of xenon under the conditions of these studies. A specially designed valve is housed in the spinner so that the polymer sample can be charged with xenon gas. Typically 120 psig 
(equivalent to 9.287 bar, total pressure) of xenon was used. Equilibration was almost instantaneous.

Variable-temperature static ${ }^{2} \mathrm{H}$ NMR data were obtained at a frequency of $30 \mathrm{MHz}$ using standard quadrupolar echo sequences. All data were acquired on a Bruker CXP-200 instrument, which had been upgraded with a Tecmag data acquisition package. For the PIB homopolymer data, 512 scans were collected, while 2000 transients were used for the PIB in the PIB/PEB-66 blend.

\section{4-4 Results and Discussion}

\section{${ }^{1} \mathrm{H}$ Observation and Relaxation}

Analysis of morphology in polymer blends may be addressed using solidstate NMR methods. In particular, non-destructive spin-diffusion techniques are attractive since isotopic labeling is not a requirement, and recent developments in the understanding of spin-diffusion data have increased the quantitative accuracy of the method. ${ }^{106-109}$ However, as we have previously shown, direct ${ }^{1} \mathrm{H}$ observation of spin-diffusion in amorphous polyolefin blends is problematic due to the lack of resolution between the inhomogeneously-broadened lines characteristic of such polymers, even with multiple pulse or fast MAS methods. ${ }^{34}$ Figure 4-1 shows ${ }^{1} \mathrm{H}$ MAS spectra obtained at $8 \mathrm{kHz}$ for the PIB/PEB-66 blend, in which an additional dipolar filter sequence is applied prior to the read pulse in order to introduce spectral resolution based on gradients in the timescale of molecular motion in the two polymers. ${ }^{110}$ Comparison of Figures 4-1(a)-(d), in which the strength of the dipolar filter is increased in subsequent spectra, shows 
that this is not achieved experimentally, thereby eliminating the possibility of direct observation spin-diffusion methods. Results were identical for experiments without MAS, except that the linewidth was larger by a factor of 6-7. As one would expect, similar results were obtained for the higher-resolution ${ }^{13} \mathrm{C}$-detected ${ }^{1} \mathrm{H}$ dipolar filter experiments, precluding the use of natural abundance spindiffusion experiments as a quantitative tool for measuring length scales of mixing in these blends. Of course, the fact that the chain dynamics of the PIB and PEB samples used in this study were too similar to allow contrast based on the dipolar filter, or Goldman-Shen methods ${ }^{111}$ (which were also attempted), does not mean this is generally the case for polyolefin blends.

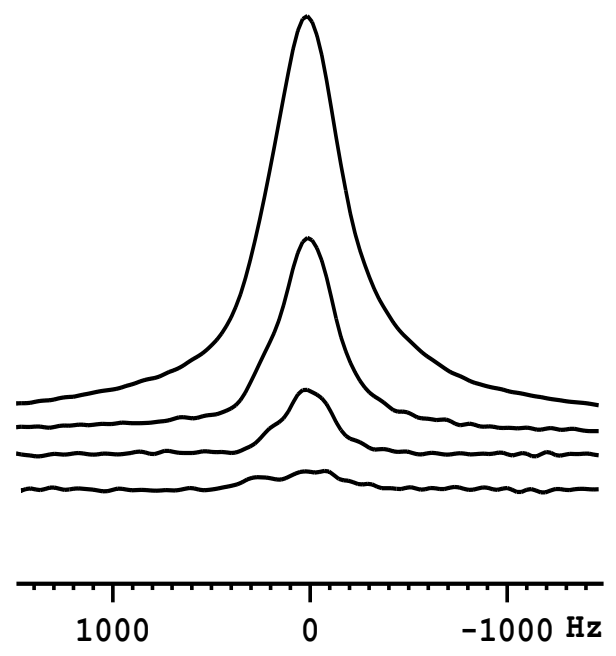

Figure 4-1 ${ }^{1} \mathrm{H}$ dipolar filter on PIB/PEB-66 blend at $23^{\circ} \mathrm{C}$ as a function of the number of 12-pulse dipolar filter cycles, in which the interpulse spacing was $10 \mu \mathrm{s}$. From top to bottom, the number of filter cycles was zero, 10,20 , and 30 , respectively.

Each blend type must be evaluated individually, and indeed, some of our previous work on polyolefin blends has successfully utilized spin-diffusion for measuring 
domain sizes. ${ }^{33}$ Preliminary experiments in which ${ }^{13} \mathrm{C}$ chemical shift filters were used to generate ${ }^{1} \mathrm{H}$ polarization gradients in one polymer, via selective DANTE pulses, suffered from unacceptably poor signal-to-noise, and were not pursued further. Such experiments will be investigated at a later time with appropriate levels of ${ }^{13} \mathrm{C}$ labeling.

The degree to which $T_{1 \rho \mathrm{H}}$ data may be used to prove intimate mixing via spin-diffusion is critically dependent upon the motional characteristics of the polymer system. For mobile polymers, it is not a reliable measure of the length scale of mixing, since the values for individual protons within a monomer unit vary greatly. This is true for PIB and PEB-66. For neat PIB, the $\mathrm{CH}_{2}$ and $\mathrm{CH}_{3}$ groups have values at $298 \mathrm{~K}$ of 520 and $1220 \mu$ s, respectively. Similarly, in neat PEB-66, the values for $\mathrm{CH}_{2}$ and $\mathrm{CH}_{3}$ groups differ by more than a factor of two. While the pure component polymer values do converge for PIB at $213 \mathrm{~K}$, a more reasonable temperature for interpreting the data due to improved spin-diffusion, they do not reach the same value. These results are in contrast to semicrystalline polyolefins, for example, where we find that all protons in the crystallites of isotactic polypropylene (i-PP) have a common $\mathrm{T}_{1 \mathrm{pH}}=20 \mathrm{~ms}$ at room temperature. For the PIB/PEB-66 blend, only the PIB methylene carbons and PEB methyl carbons are well-resolved at $213 \mathrm{~K}$, and their $\mathrm{T}_{1 \mathrm{pH}}$ values are 498 and $1100 \mu \mathrm{s}$, respectively. We point out these results here since the observation of disparate $\mathrm{T}_{1 \rho \mathrm{H}}$ data is often used to "prove" that blends are not intimately mixed. Obviously, this criterion is not applicable to amorphous polyolefin blends, since 
the neat polymers themselves have unique values within the monomer unit, even at temperatures near $\mathrm{T}_{\mathrm{g}}$.

\section{Variable-temperature $1 \mathrm{D}^{13} \mathrm{C}$ CP/MAS NMR Data}

Results from variable-temperature $\mathrm{CP} / \mathrm{MAS}$ experiments on $\mathrm{PIB}$ and its blends with PEB are shown in Figure 4-2. Details concerning the relative rates of conformational exchange along the backbone of PIB are revealed by the lineshape changes of the PIB $\mathrm{CH}_{2}$ signal as the temperature is increased from $213 \mathrm{~K}\left(10^{\circ}\right.$ above the DSC $\mathrm{T}_{\mathrm{g}}$ ). At $213 \mathrm{~K}$ in Figure 4-2(a), the broad $\mathrm{CH}_{2}$ line for neat PIB, extending from 45 to $80 \mathrm{ppm}$ (see inset expansions), has resolved features corresponding to segments in trans-trans, trans-gauche, or gauche-gauche conformations. $^{42}$ As the temperature is increased, that $\mathrm{CH}_{2}$ lines broaden, becomes featureless, and reappears as a single line at room temperature. The temperature at which the line for neat PIB is completely broadened is $267 \mathrm{~K}$ (spectrum not shown), near the 263 and $273 \mathrm{~K}$ data points shown in 4-2(a). The broadening mechanism is the well-known Rothwell-Waugh effect, where incoherent polymer chain motion occurs on a similar timescale as the coherent averaging r.f. field that is applied to decouple the protons. ${ }^{56}$ When the frequency of chain motions approaches that of the r.f. field strength or the MAS rate, line widths in the ${ }^{13} \mathrm{C}$ spectrum may become broad beyond detection. Indeed, this effect is commonly observed in many polymers, but usually for $\mathrm{CH}_{3}$ carbons. In this case, the results in 4-2(a) indicate that the timescale of conformational exchange rates along the PIB backbone, i.e. between $\mathrm{t}-\mathrm{t}, \mathrm{t}-\mathrm{g}$, and $\mathrm{g}$-g conformers, is centered about $73 \mathrm{kHz}$ at $267 \mathrm{~K}$. Of course, there is indeed a distribution for 
motional correlation frequencies, but the fact that a completely flat $\mathrm{CH}_{2}$ "signal" is observed at $267 \mathrm{~K}$ indicates that the majority of the polymer chains satisfy this condition.
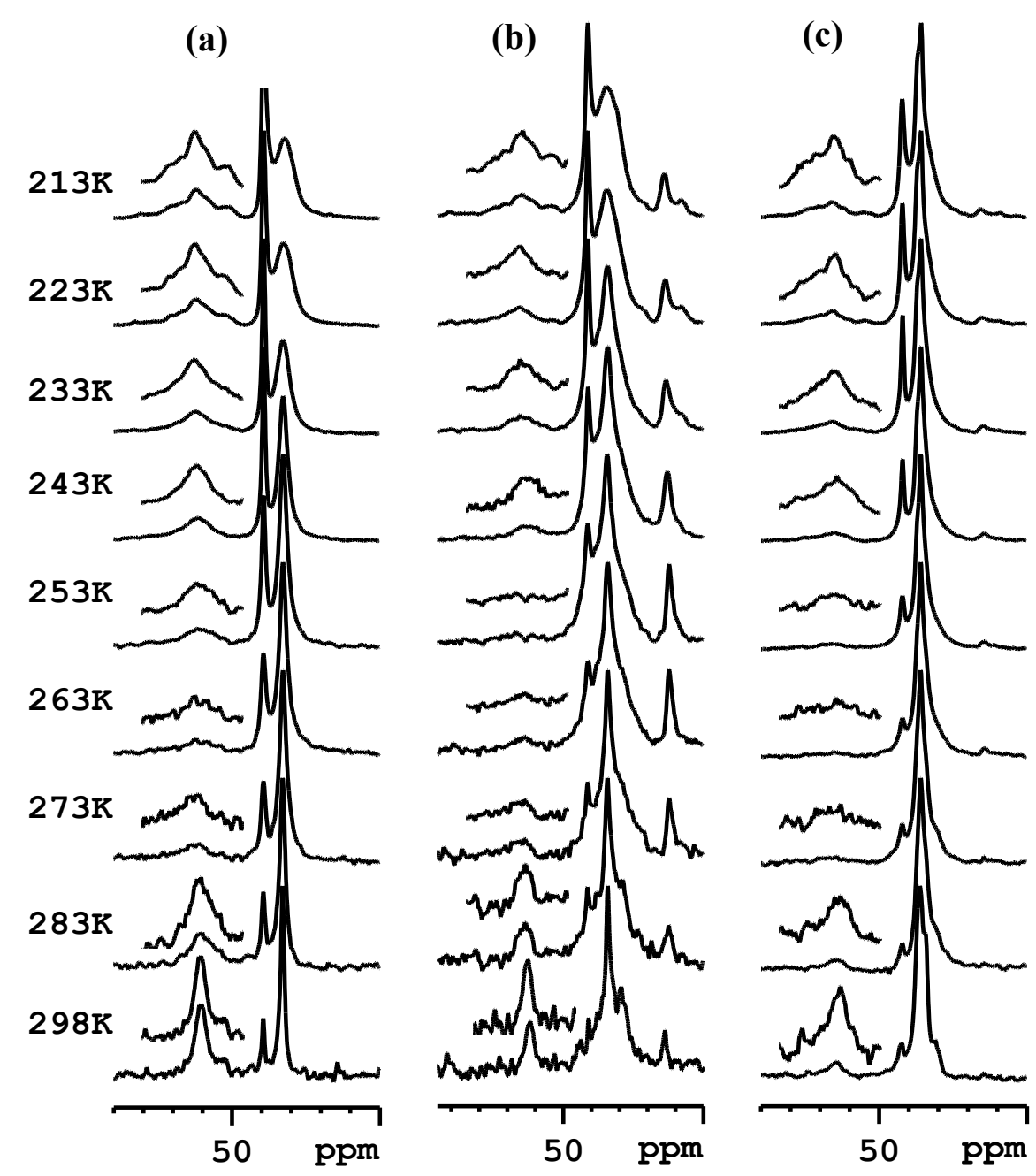

Figure 4-2 Variable-temeperature CP/MAS stack plot of (a) PIB, (b) PIB/PEB-66 blend, and (c) PIB/PEB-23 blend. Acquisition temperatures are indicated on the figure, and the $\mathrm{PIB} \mathrm{CH}_{2}$ region is expanded in the inset above each spectrum. Note that $\mathrm{PIB} \mathrm{CH}_{2}{ }^{1} \mathrm{H} \mathrm{T}_{1 \rho}$ relaxation time constants at $213 \mathrm{~K}$ are $810 \mu \mathrm{s}, 498 \mu \mathrm{s}$, and $884 \mu \mathrm{s}$ for the samples in (a), (b), and (c), respectively. These results agree with the lineshape data.

In contrast, Figure 4-2(b) shows the results from the same experiment for the $\mathrm{PIB} \mathrm{CH}_{2}$ group in the blend with PIB-66. Immediately one observes that the 
temperature at which the maximum interference of $\mathrm{PIB} \mathrm{CH}_{2}$ motion with the decoupling field occurs is $253 \mathrm{~K}$ in the blend, some $10-15 \mathrm{~K}$ lower than in the neat PIB. Again, the lineshape changes unequivocally indicate that the relevant exchange process is conformational exchange between backbone conformers, a process that based on the comparison of 4-2(a) and 4-2(b), occurs at significantly lower temperatures for PIB in the blend with PEB-66. For example, one still observes a "rigid" $\mathrm{CH}_{2}$ lineshape for the neat PIB at $233 \mathrm{~K}$, while the $\mathrm{CH}_{2}$ signal for PIB in the blend at $233 \mathrm{~K}$ is superposable with the $253 \mathrm{~K}$ signal from neat PIB. Also, complete inspection of PIB/PEB-66 plots in 4-2(b) shows that the breadth of the transition as a function of temperature is significantly wider in the blend. By comparison, the $\mathrm{CH}_{2}$ lineshape for the PIB/PEB-23 blend in Figure 42(c) much more closely mimics the temperature dependence of the neat PIB, e.g., resolved conformer contributions to the lineshape persist up to $243 \mathrm{~K}$, and signal is still observed at $253 \mathrm{~K}$, in contrast to the PIB/PEB-66 blend.

\section{Static $^{2}$ H NMR}

${ }^{2} \mathrm{H}$ solid-state NMR can provide very specific details concerning the nature, the amplitude, and the rates of molecular motions. ${ }^{112}$ Figure 4-3 shows selected results from a complete variable-temperature study on PIB and the two blends, in which the temperature was increased in $10 \mathrm{~K}$ increments from $210 \mathrm{~K}$. In 4-3(a) - 4-3(d), the spectrum from neat perdeuterated PIB is shown on top, and the PIB/PEB- 66 blend on the bottom. Only $20 \%$ of the PIB monomer units were perdeuterated in the blend sample. Since the PIB units are perdeuterated, the spectra are dominated by the motionally-averaged $\mathrm{CD}_{3}$ signal; the broad $\mathrm{CD}_{2}$ 
pattern $(135 \mathrm{kHz}$ splitting) is well-resolved only in the $210 \mathrm{~K}$ spectrum. As such, these data on specific $\mathrm{CH}_{3}$ dynamics provide a compliment to the $\mathrm{CH}_{2}$-specific results from the ${ }^{13} \mathrm{C}$ experiments. Rapid $\mathrm{C}_{3}$ rotation of the methyl group provides an anisotropically averaged Pake pattern with a $40 \mathrm{kHz}$ splitting, even at $210 \mathrm{~K}$. Additional reduction of this splitting will only occur if additional modes of

(a)

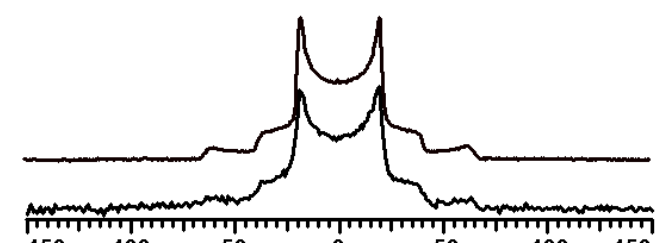

(b)

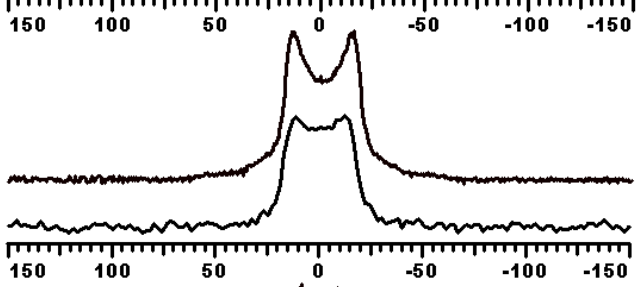

(c)
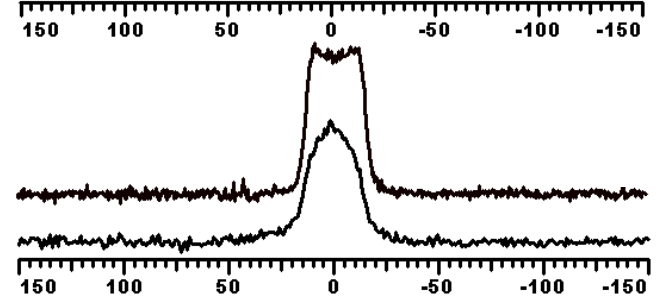

(d)

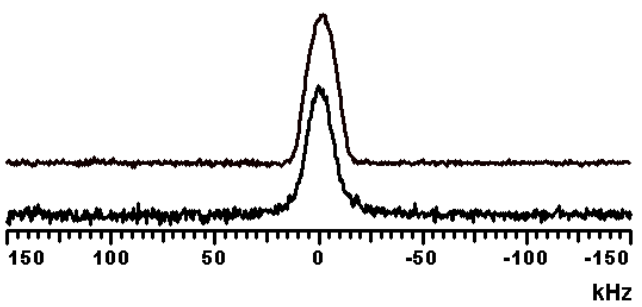

Figure 4-3 Selected spectra from variable-temperature static ${ }^{2} \mathrm{H}$ experiments on PIB- $\mathrm{d}_{8}$ (top) and PIB( 18 mole $\left.\%-\mathrm{d}_{8}\right) / \mathrm{PEB}-66$ blend (bottom) at (a) $210 \mathrm{~K}$, (b) $250 \mathrm{~K}$, (c) $260 \mathrm{~K}$, and (d) $270 \mathrm{~K}$. Although only four data points are shown, spectra were collected at $10 \mathrm{~K}$ increments from $210 \mathrm{~K}$ to $300 \mathrm{~K}$. Note the faster motional timescale of the PIB chains in the blend relative to bulk PIB at the three higher temperatures.

motion are present, which reorient the bond vector between the methyl and backbone carbon in space. For the case of PIB, these additional motions will only arise through motions associated with the polymer backbone, since they are 
attached to quaternary backbone carbons. Therefore, changes in the $\mathrm{CD}_{3}$ lineshape should also be sensitive to conformational dynamics in PIB. Figure 4-3 shows additional narrowing, and ultimately, collapse of the $\mathrm{CD}_{3}$ lineshape for the neat polymer, and the PIB in the blend with PEB-66 at $270 \mathrm{~K}$. Additional temperature increases result in further narrowing of the line, but they are not shown here. More interesting is the marked contrast in the lineshape between the two samples at each temperature. In particular, at $260 \mathrm{~K}$, the neat PIB lineshape is still not coalesced, while the PIB in the blend is past the coalescence point. At $270 \mathrm{~K}$, the PIB linewidth is $25 \mathrm{kHz}$ at half-height, while that of the PIB in the blend is $15 \mathrm{kHz}$. Clearly, the motional frequency of backbone dynamics in PIB is increased at a given temperature, relative to the neat polymer. We are currently working on specific motional models for simulating the ${ }^{2} \mathrm{H}$ lineshape results, as well as preparing PIB polymer specifically labeled at the $\mathrm{CH}_{2}$ position. Even so, we see that there is excellent agreement in the coalescence temperatures from the methyl ${ }^{2} \mathrm{H}$ spectra in Figure 4-3 and the methylene ${ }^{13} \mathrm{C}$ spectra in Figure 4-2. For the blend, that temperature is approximately $253 \mathrm{~K}$, and for the neat PIB, $265 \mathrm{~K}$. Although the PIB is an amorphous polymer, with a distribution of chain dynamics, it is satisfying that both the ${ }^{13} \mathrm{C}$ and ${ }^{2} \mathrm{H}$ NMR results provide a consistent explanation of increased conformational chain freedom for the PIB/PEB-66 blend.

\section{Two-Dimensional ${ }^{13} \mathrm{C}$ Exchange Experiments}

One-dimensional lineshape experiments, like those in Figures 4-2 and 43, provide qualitative insights into the differences between PIB chain dynamics. 
Quantitative analysis of exchange lifetimes, and identification of conformational exchange pathways, requires 2D MAS exchange experiments. ${ }^{36,42,48}$ In Figure 44, results from rotor-synchronized $2 \mathrm{D}{ }^{13} \mathrm{C}$ homonuclear exchange experiments are
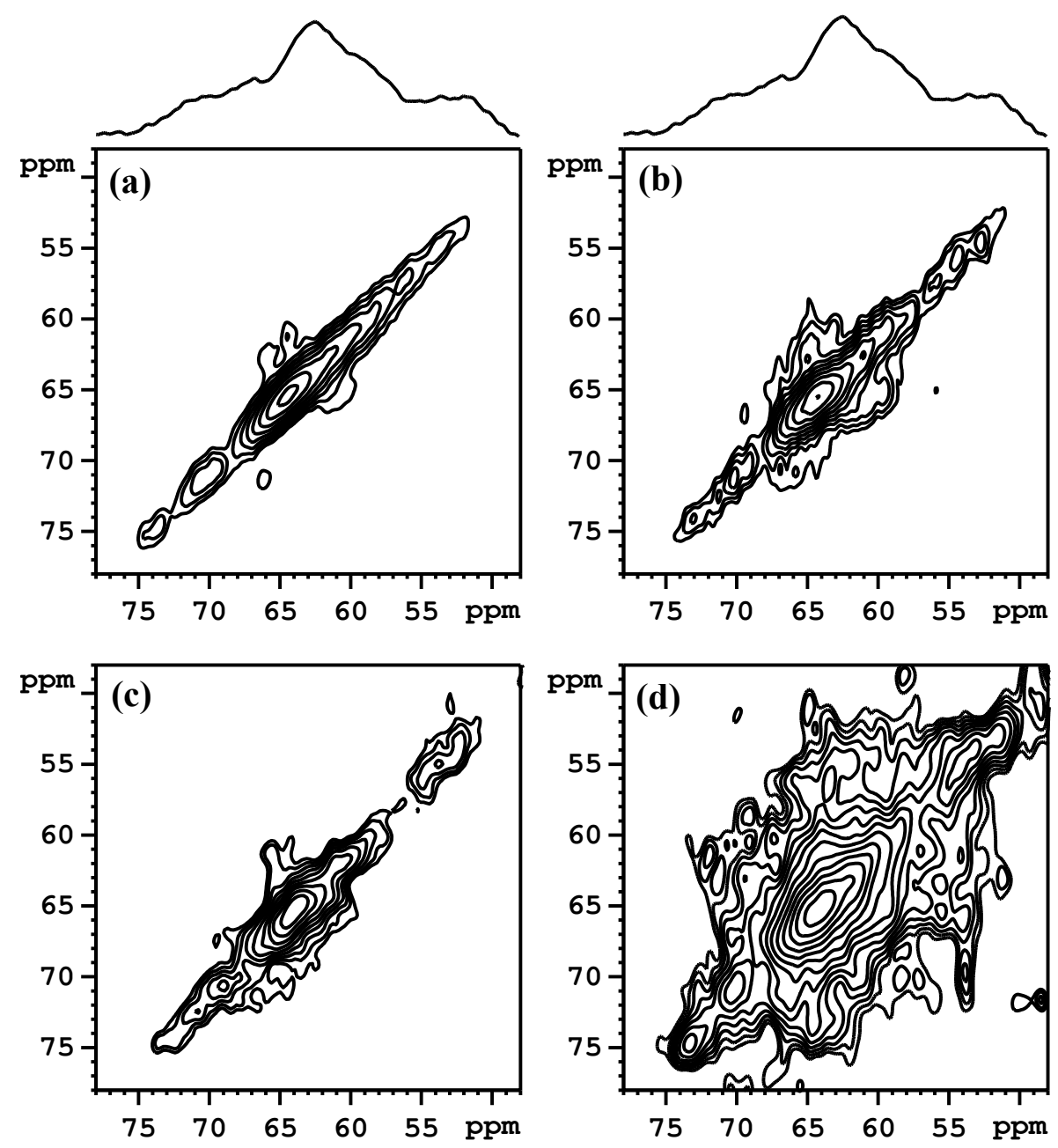

Figure 4-4 Two-dimensional MAS ${ }^{13} \mathrm{C}$ exchange spectra of the PIB methylene region (50-80 ppm) at $213 \mathrm{~K}$ for (a) neat PIB with 100 millisecond mixing time, (b) neat PIB with 1 second mixing time, (c) PIB/PEB-23 blend with 1.5 second mixing time, and (d) PIB/PEB-66 blend at 100 -ms mixing time. ${ }^{13} \mathrm{C} \mathrm{T}_{1}$ values at $213 \mathrm{~K}$ equal $2.9 \mathrm{~s}$ for the $\mathrm{PIB}_{\mathrm{CH}_{2}}$ carbons in all samples.

for PIB and the two blends with PEB. These data were acquired at $213 \mathrm{~K}$, or $8 \mathrm{~K}$ shown above the glass transition for pure PIB as measured by DSC. Figure 44(a) shows the control experiment on neat PIB with a short 100-ms mixing time, 
and as expected, there is no off-diagonal intensity. After 1 second (4-4(b)), the diagonal is broadened slightly, and the signal-to-noise decreases slightly due to spin-lattice relaxation during the mixing time $\left(\mathrm{T}_{1}=2.9 \mathrm{~s}\right.$ for PIB in bulk and in blends). However, again we see little off-diagonal intensity that would suggest conformational exchange even after 1 second. Within the limits of the sensitivity, similar results are observed for the PIB $\mathrm{CH}_{2}$ group in the blend with PEB-23; a well-resolved diagonal dominates the 2D spectrum in 4-4(c) after $1.5 \mathrm{~s}$ of exchange. In clear contrast to any of these results, diagonal intensity is completely redistributed among all conformers for the $\mathrm{CH}_{2}$ group of $\mathrm{PIB}$ in the PIB/PEB-66 blend (4-4(d)) after only $100 \mathrm{~ms}$. In this blend, the $\mathrm{CH}_{2}$ carbons of PIB have been labeled with $5 \%{ }^{13} \mathrm{C}$ to increase sensitivity. A similar exchange pattern, albeit at lower sensitivity, was obtained for the sample in 4-4(d) after a 1-s exchange period (not shown). Comparison of slices from the 100-ms experiment in 4-4(d) and the 1-s experiment (not shown) revealed essentially complete exchange in each. More importantly, comparison of 4-4(d) with 4-4(a) and 4-4(b) shows that exchange rate is much larger for PIB in the miscible blend, relative to the pure PIB. We also note that these results agree with $T_{1 \rho H}$ data on PIB and the two PEB blends at $213 \mathrm{~K}$. The $\mathrm{CH}_{2}$ protons in neat PIB and PIB in the PIB/PEB-23 blend have similar values of $810 \mu$ s and $884 \mu$ s, respectively, while the PIB in the PIB/PEB-66 blend has $\mathrm{T}_{1 \rho \mathrm{H}}=498 \mu \mathrm{s}$. By comparison, we do not observe such a short value of $\mathrm{T}_{1 \rho \mathrm{H}}$ for neat PIB until the temperature is increased to $298 \mathrm{~K}$, at which point the neat PIB $\mathrm{T}_{1 \rho \mathrm{H}}=516 \mu \mathrm{s}$. The conformational freedom of PIB 
chains in the blend with PEB-66 is much larger at any given temperature than in neat PIB, or the PIB/PEB-23 blend.

In an effort to verify that the exchange pattern progressed as expected with longer mixing times, $\mathrm{PIB} / \mathrm{PEB}-66$ results for the $5 \%$ labeled $\mathrm{PIB} \mathrm{CH}_{2}$ moeties (in the blend) are shown in Figure 4-5. Only 64 scans were collected for each of these, which explains the lower sensitivity relative to the 256 scans used in Figure 4-4(d). In addition to quantitative exchange rates, we are interested in identifying possible pathways for exchange, e.g., if one could observe sequential events in which $\mathrm{tt} / \mathrm{tg}$ and $\mathrm{tg} / \mathrm{gg}$ exchange occurred before the detection of $\mathrm{tt} / \mathrm{gg}$ exchange. This requires running several experiments at relatively short mixing times. Figure 4-5(a) shows the results for a short mixing time of $5 \mathrm{~ms}$, in which no exchange is observed, while Figures 4-5(b), 4-5(c), and 4-5(d) correspond to mixing time of 25, 50, and $100 \mathrm{~ms}$, respectively. As off diagonal intensity develops within the tg and between $\operatorname{tg} / \mathrm{gg}$ peaks, we also observe the loss of the weak diagonal peak corresponding to the $t$ conformers $(67-75 \mathrm{ppm})$. These results indicate that, within the limits of the sensitivity, exchange is occurring with approximately the same rate between all possible conformers. By $100 \mathrm{~ms}$ (45(d)), one can discern an approximately fully developed exchange pattern, particularly between the tg/gg regions. Of course, as diagonal intensity is redistributed among all possible off-diagonal elements, and spin-lattice relaxation takes place, the overall sensitivity decreases, as shown for the 1.5 second result in 4-5(e). 
(a)
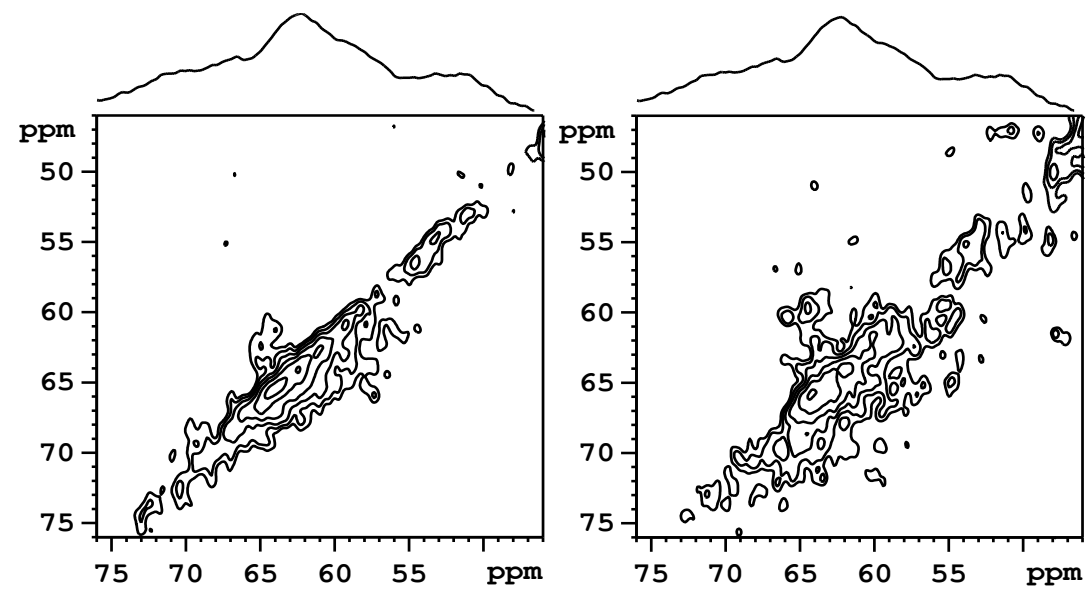

(b)

(c)
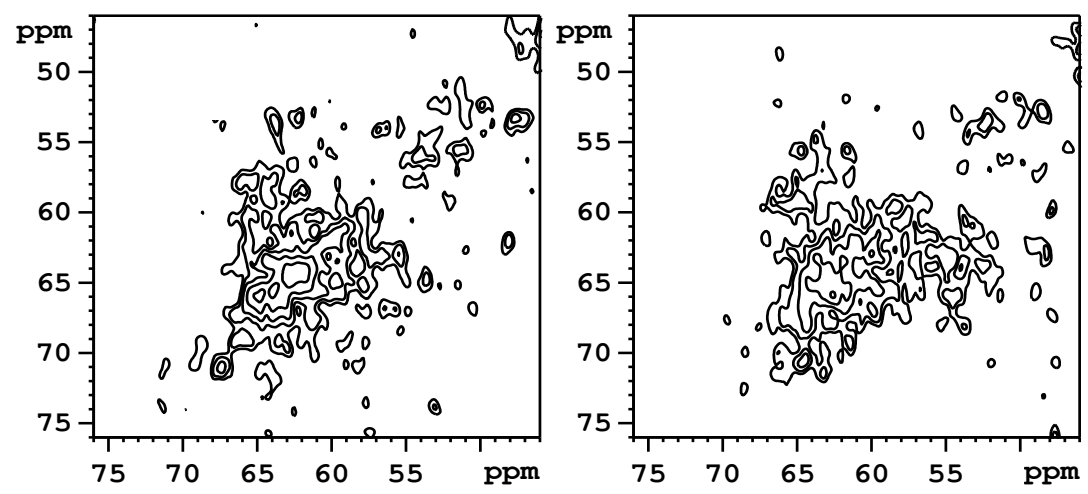

(d)

(e)

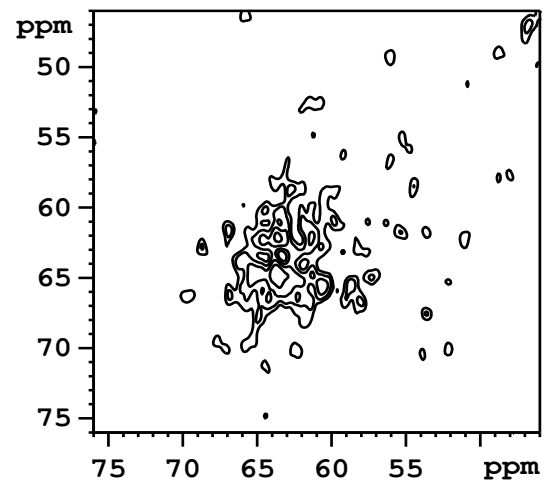

Figure 4-5 Two-dimensional MAS ${ }^{13} \mathrm{C}$ exchange spectra of the PIB methylene region (50-80 ppm) at $213 \mathrm{~K}$ for a PIB/PEB-66 blend in which the PIB contains $5 \%{ }^{13} \mathrm{C}$-labeled $\mathrm{CH}_{2}$ groups at mixing times of (a) $5 \mathrm{~ms}$, (b) $25 \mathrm{~ms}$, (c) $50 \mathrm{~ms}$, (d) $100 \mathrm{~ms}$, and (e) 1 second.

\section{${ }^{129}$ Xe of Xenon Gas Absorbed in PIB and PIB/PEB Blends}

NMR of Xe gas, which is absorbed into a polymer blend, has been shown to be a good indicator of whether the polymer is homogeneously mixed on a length scale defined by the Xe gas diffusion coefficient. ${ }^{78,113}$ Due to its large, 
highly polarizable electron density, the ${ }^{129} \mathrm{Xe}$ chemical shift is very sensitive to subtle changes in its electronic environment. In amorphous polymers, the free volume of the polymer largely determines the ${ }^{129} \mathrm{Xe}$ chemical shift. We have used this non-destructive method to provide complimentary information to the dynamic NMR data presented above. Figure 4-6 presents the results of simple ${ }^{129} \mathrm{Xe}$ onepulse experiments for Xe gas absorbed in PIB and its blends with PEB-66 and PEB-23. Figure 4-6(b) shows that a single peak with an intermediate chemical shift is observed in the PIB/PEB-66 blend, relative to the neat PIB and PEB-66 in 4-6(a) and 4-6(c), respectively. In contrast, Figure 4-6(e) shows two peaks at the

(a)

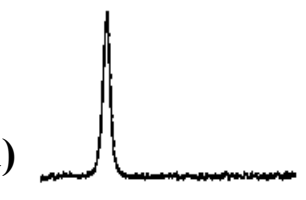

(b)

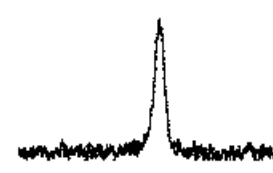

(c)

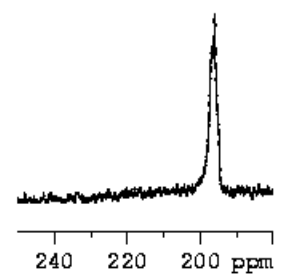

(d)

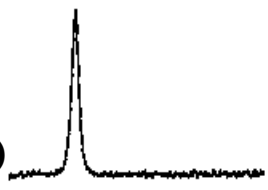

(e)

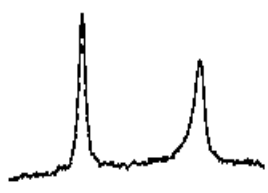

(f)

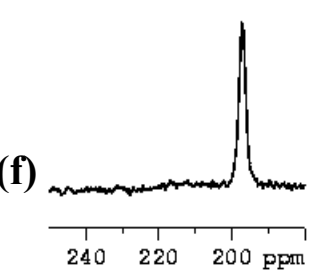

Figure 4-6 Static ${ }^{129} \mathrm{Xe}$ NMR spectra of Xe gas adsorbed in (a) PIB, (b) PIB/PEB-66 blend, (c) PEB-66, (d) PIB, (e) PIB/PEB-23 blend, and (f) PEB-23. The number of transients vary in each case, but are greater than $1 \mathrm{~K}$ for all. The mass of each sample also varies between experiments.

chemical shift of the pure polymers for the PIB/PEB-23 blend, as may be observed by comparing its spectrum with those in 4-6(d) and 4-6(f). These data clearly indicate that the PIB/PEB-66 blend is miscible on a length scale defined 
by the chemical shift difference of the two constituents, and the Xe gas selfdiffusion coefficient $\mathbf{D}_{\mathbf{X}}$ in these low- $T_{\mathrm{g}}$ polymers. An estimate can be made concerning the upper limit of the length scale of mixing, or domain sizes, in the fast exchange limit shown by Figure 4-6(b). The lifetime of a Xe gas molecule $\tau$ $<<(1 / \Delta v)$ for the fast exchange regime, where $\Delta v=4841 \mathrm{~Hz}$ is the difference between the pure component shifts. Therefore, the average distance a Xe gas molecule diffuses in a time $\tau$ is:

$$
x_{r m s}<<\sqrt{\frac{2 D_{X e}}{\Delta v}}
$$

Since we do not have the experimental capability to directly determine $\mathbf{D}_{\mathbf{X e}}$ using PFG methods, values from the literature for similar low- $T_{g}$ polymers are used in above equation. Following the reports by Walton ${ }^{113}$ and Veeman ${ }^{78}, \mathbf{D}_{\mathbf{X e}}=10^{-7}$ $\mathrm{cm}^{2} / \mathrm{s}$ is taken as a reasonable upper limit in these polyolefins. Solving, we obtain $\mathbf{x}_{\mathrm{rms}}<<64 \mathrm{~nm}$ as an upper limit on the domain size of the PIB/PEB-66 blend. However, we point out that this is indeed a maximum limit, and remind the reader that the $1 \mathrm{D}$ and $2 \mathrm{D}$ exchange experiments presented above indicate that the length scale of mixing is actually much shorter. For reference, the $64 \mathrm{~nm}$ limit is almost an order of magnitude larger than $\mathrm{R}_{\mathrm{g}}$ for these chains, and in order for mixing to induce large changes in the PIB backbone dynamics, the local environment around each chain must be perturbed.

\section{Implications for Thermodynamics of Mixing in Saturated Polymer Blends}

The direct experimental evidence presented above indicates that (1) of the two blends, only the PIB/PEB-66 blend is miscible, and (2) the barrier to 
conformational exchange of PIB chains in the miscible blend is significantly lower than in pure PIB. At $213 \mathrm{~K}$, all three conformational states of PIB can freely exchange between one another with a rate larger than $1 \mathrm{kHz}$, while the pure PIB exchange rates are negligible. (Indeed, we did not observe any conformational exchange in neat PIB or the PIB/PEB-23 blend up to the 2-second maximum exchange time used in this study). The ${ }^{2} \mathrm{H}$ and ${ }^{13} \mathrm{C} 1 \mathrm{D}$ lineshape, ${ }^{13} \mathrm{C}$ $2 \mathrm{D}$ exchange, and $\mathrm{T}_{1 \mathrm{pH}}$ data all indicate that this is the case. In terms of the absolute entropy $\mathbf{S}$ of the PIB chains in the PEB-66 blend, in which there are 3 chain conformations/backbone bond relative to only one in the pure PIB, the ratio of $\mathbf{S}_{\text {blend }} / \mathbf{S}_{\text {pure }}=\ln \left(\mathbf{3}^{\mathbf{N}}\right)$, where $\mathbf{N}$ is the number of backbone bonds in the PIB chain. However, this only accounts for the entropy change for PIB in the blend. One must also consider the effects of blending on the PEB-66 polymer. Examination of the 10-15 ppm region in Figure 4-2 shows that the $\mathrm{CH}_{3}$ groups of the ethyl side chains in PEB-66 are sensitive to conformation, since resolved trans-gauche/gauche-gauche peaks are observed below $243 \mathrm{~K}$. Comparison of the temperature dependence of the $\mathrm{CH}_{3}$ peaks in the blend with the neat PEB-66 copolymer, over the entire temperature range (223-253 K in particular), indicates that the conformational dynamics are similar in each. The peaks are superimposable at each temperature with those for the neat PEB-66 (not shown). Since the $1 \mathrm{D}{ }^{13} \mathrm{C}$ lineshape changes with temperature agreed with the detailed $2 \mathrm{D}$ exchange data for the PIB methylene carbons, we are confident that similar information for the PEB-66 methyl group indicates little change in its chain dynamics upon blending. Clearly, the PEB-66 is not more "constrained" at a 
local chain level in the blend relative to its pure state. On the whole, these results suggest that configurational entropy may be the operative driving force in the overall negative $\Delta \mathrm{G}$ required for miscibility.

Such a result is intuitively satisfying for blends involving PIB, since the chain packing density in pure PIB is higher relative to other simple polyolefins, and it is unlikely that PIB packs more efficiently with dissimilar chain architectures. ${ }^{114}$ Indeed, there have been several reports that PIB displays unusual mixing behavior compared to most polyolefins, and qualitatively this could be explained by increases in the absolute configurational entropy $\mathbf{S}=\mathbf{k}_{\mathbf{B}} \ln \mathbf{W}$ upon mixing. Miscibility between macromolecules is rarely attributed to entropic contributions, as Flory-Huggins theory indicates that these will scale to zero with

typical polymer molecular weights. ${ }^{115}$ However, such arguments are based on consideration of a molecular "size parameter", such as the degree of polymerization or molecular mass, and fail to recognize the dynamic heterogeneity that exists on a short length scale among the sub-units in the chain. Of course at this stage, these are qualitative arguments, but they are in complete agreement with the observed experimental findings for the PIB/PEB-66 blend.

\section{4-5 Conclusions}

Direct experimental observation of local chain dynamics, and mixing, in blends of PIB and PEB indicate that these polymers are not miscible over the entire butene comonomer range. For 50/50 blends, we observe intimate chain mixing for PIB and PEB containing $66 \mathrm{wt} \%$ (49 mole\%) butene comonomer, but 
phase separation for a PIB/PEB-23 blend. A variety of site-specific 1D and 2D dynamic solid-state NMR experiments, most in the absence of isotopic labeling, indicated that the conformational exchange rates of the PIB backbone increase significantly upon blending with the PEB-66 blend, while the PEB-66 itself was not affected by blending. Longer range probes of blend structure using Xe gas absorption revealed that the PIB/PEB-66 blend was homogeneous on a length scale $<<64 \mathrm{~nm}$, in agreement with the chain-level NMR results. In addition to demonstrating an experimental strategy by which one can generally probe polyolefin blend mixing at the chain level, our results suggest that local configurational entropy may be the operative driving force for miscibility in polyolefin blends. 
Chapter 5:The Glass Transition Time Scale and Configurational Entropy in Polymers: An Experimental Molecular View

\author{
J. E. Wolak, X. Jia, and Jeffery L. White \\ Department of Chemistry, North Carolina State University, Campus Box 8204, \\ Raleigh, NC 27695-8204
}

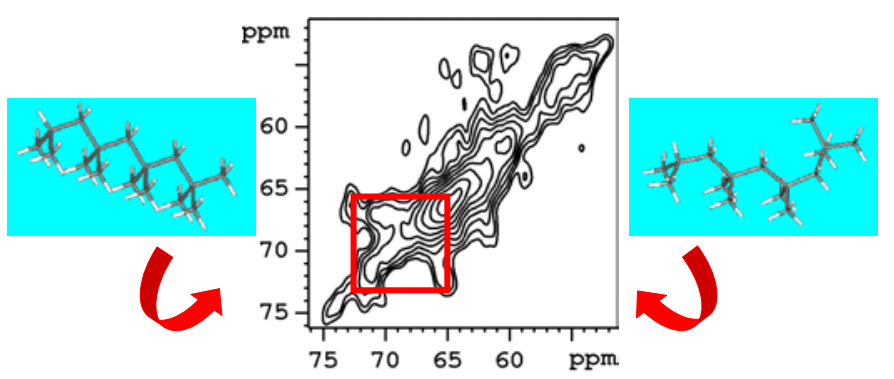

As published in the Journal of American Chemical Society, 2003, Vol.125,

p.13660-13661 


\section{5-1 Abstract}

We report direct experimental observation of local conformational dynamics in a polymer chain at the calorimetric glass transition temperature $T_{g}$. Variable-temperature 2D solid-state exchange NMR, at natural abundance, reveals segmental dynamics in pure polyisobutylene (PIB) occurring on a timescale of several seconds over the $\mathrm{T}_{\mathrm{g}}$ range observed by DSC (203-208 K). To our knowledge, this is the first direct observation of molecular-level conformer interchange (trans-trans/trans-gauche/gauche-gauche) at the caloric glass transition temperature. Our results provide a chronologically accurate and pedagogically advantageous demonstration of molecular processes during a polymer phase transition, relative to traditional bulk mechanical and calorimetric techniques. More importantly, we use a miscible blend to demonstrate a general strategy for quantitative evaluation of configurational entropy changes via combination of temperature-dependent 2D exchange NMR and Adams-Gibbs theory. Our results on the $\mathrm{Tg}$ timescale are directly relevant to fundamental understanding of the $\mathrm{T}_{\mathrm{g}}$ length-scale, i.e., the dimension of cooperatively rearranging regions.

\section{5-2 Introduction}

Molecular processes involving slow conformational exchange are assumed to give rise to glass to liquid transitions in synthetic high polymers. The timescale and length-scale of both individual segmental dynamics and correlatedchain, or cooperatively rearranging, motions is currently receiving widespread 
attention in the literature. ${ }^{116-118} \mathrm{~A}$ variety of experimental methods, ranging from bulk calorimetry and mechanical methods to neutron scattering and positron annihilation spectroscopies, comprise the current arsenal devoted to this fundamental problem in materials science. ${ }^{119}$ Spiess and coworkers have demonstrated that solid-state exchange NMR is particularly useful for interrogating polymer chain dynamics above the calorimetric glass transition

temperature $\mathrm{T}_{\mathrm{g}}{ }^{9}{ }^{9}{ }^{42,}, 61,120-123$ In each case, dynamics were observed on timescales of less than $1 \mathrm{~s}$, for temperatures at least $8 \mathrm{~K}$ above the calorimetric $\mathrm{T}_{\mathrm{g}}$. In this communication, we examine the timescale for conformer interchange at the calorimetric $T_{g}$ for polyisobutylene (PIB) using $2 \mathrm{D}{ }^{13} \mathrm{C}$ exchange NMR, and demonstrate that the timescale of the $T_{g}$ process is on the order of several seconds.

\section{5-3 Results and Discussion}

Figure 5-1 illustrates the vastly different information content obtained from the traditional differential scanning calorimetry (DSC) $T_{g}$ experiment versus that from $2 \mathrm{D}{ }^{13} \mathrm{C}$ exchange NMR. The DSC trace for PIB shown in Figure 5-1 was collected using a $2 \mathrm{~K} / \mathrm{min}$ scan rate, resulting in a broad transition spanning a $5 \mathrm{~K}$ range $(203-208 \mathrm{~K})$. Other than suggesting a heterogeneous distribution of heat capacities, the DSC result provides little information concerning the molecular dynamics, or their timescales, involved in the transition. Figures 51(a)-(c) and 5-1(d)-(f) show the $2 \mathrm{D}$ MAS ${ }^{13} \mathrm{C}$ exchange results for the main-chain $\mathrm{CH}_{2}$ group in $\mathrm{PIB}$ at temperatures corresponding to the onset $(203 \mathrm{~K})$ and completion $(208 \mathrm{~K})$ of the DSC $\mathrm{T}_{\mathrm{g}}$, respectively. (Temperatures in the 4-mm 
MAS probe were calibrated using the $\mathrm{PbNO}_{3}$ chemical shift thermometry method, ${ }^{44}$ and are accurate to within $\pm 1 \mathrm{~K} ; 2000 \mathrm{~L} / \mathrm{min}$ cooling gas flow rates were used to minimize temperature gradients in the sample.) PIB is particularly advantageous for this type of experiment, since its ${ }^{13} \mathrm{C}$ spin-lattice relaxation time
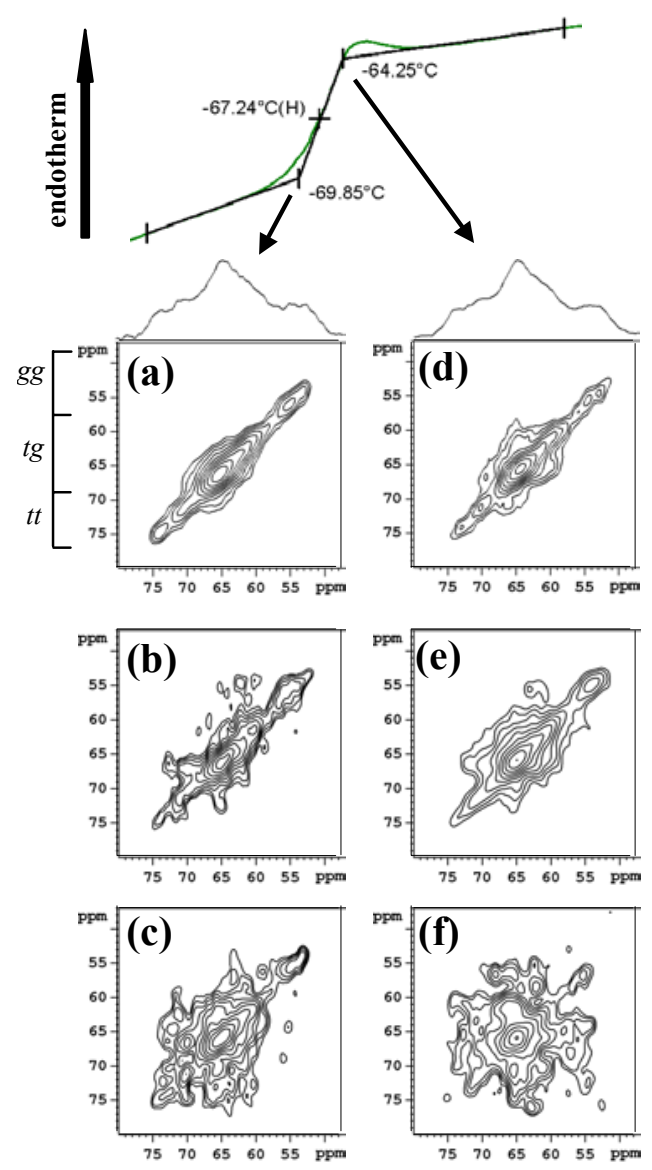

Figure 5-1 Comparison of DSC $\mathrm{T}_{\mathrm{g}}$ trace obtained with a $2 \mathrm{~K} / \mathrm{min}$ scan rate (top) with $2 \mathrm{D}{ }^{13} \mathrm{C}$ exchange spectra at (a) $203 \mathrm{~K}$ and $1 \mathrm{~s}$ mix time; (b) same as (a) at $2 \mathrm{~s}$ mix; (c) same as (a) at $4 \mathrm{~s}$ mix; (d) $208 \mathrm{~K}$ at $1 \mathrm{~s}$ mix time; (e) same as (d) at $2 \mathrm{~s}$ mix; (f) same as (d) at $4 \mathrm{~s}$ mix. Spinning speeds were $4 \mathrm{kHz}$.

constant $\mathrm{T}_{1}=3.0 \mathrm{~s}$ at $205 \mathrm{~K}$, thereby preserving signal for longer mixing times, and the chain displays all three possible conformer environments. Three different chemical exchange times are shown at each temperature, and the 1D CP/MAS 
spectrum for this $\mathrm{CH}_{2}$ resonance is shown above each contour plot. The broad peak reflects the distribution of $\mathrm{CH}_{2}$ groups for chain segments in trans-trans ( $\mathrm{tt}$ ), trans-gauche (tg), or gauche-gauche (gg) conformations along the polymer backbone. In agreement with previous reports, little or no exchange is observed for a 1-s mix time (or less) at 203 or $208 \mathrm{~K}$, as indicated by the lack of any offdiagonal intensity. ${ }^{120,121}$ However, for longer mixing times, exchange events are observed between the individual $\mathrm{tt}$, tg, and gg peaks, as shown by the increased off-diagonal intensity at the 2 and 4-s mixing times. Examination of the ratio of an individual peak width perpendicular to the diagonal, vs. parallel to the diagonal, shows that conformer exchange is essentially complete after 4s at $208 \mathrm{~K}$ (see 5-1(f)). While the amount of conformational exchange is clearly reduced in 5-1(a)-(c) vs. 5-1(d)-(f), chain segment dynamics are observed at both temperatures for the 2 and 4 -s mixing times.

The 2-s exchange time in Figure 5-1(b) indicates a surprising result, in that the first process at the glass transition onset appears to involve exchange between a small subset of tt and tg conformers (65-75 ppm region). While each diagonal peak in Figure 5-1(b) is broad, reflective of the inhomogeneous distribution of longer-range conformer sequences, relatively narrow off-diagonal ridges are observed at 2-s. In contrast, the same exchange time in 1e shows a larger and more diffuse off-diagonal intensity between $t \mathrm{t}$ and tg segments, and also within the tg peak region (60-67 ppm). The fact that the exchange patterns exhibit increased off-diagonal intensity as the temperature is increased rules out dipolar-mediated spin-diffusion as the operative spin-exchange pathway. (This 
was further confirmed by the comparison of $2 \mathrm{D}$ experiments at $\mathrm{T}_{\mathrm{g}}$ with and without ${ }^{1} \mathrm{H}$ spin decoupling during the mixing time, in which identical exchange patterns were observed.) We believe that the NMR data provided here, in contrast to scan-rate specific calorimetric endotherms, gives the basis for a functional definition of the glass transition as that temperature and time range over which isolated chain segments access all possible conformer states, versus a subpopulation of lowest energy conformers.

The site-specific dynamics revealed by the $2 \mathrm{D}$ exchange experiment indicate changes in local glass-transition temperature/timescale upon formation of a miscible polymer blend. Figure 5-2(a) and 5-2(b) show results obtained at 208 $\mathrm{K}$ for a pure PIB sample and a 50:50 wt\% blend of PIB and polyethylene-co-
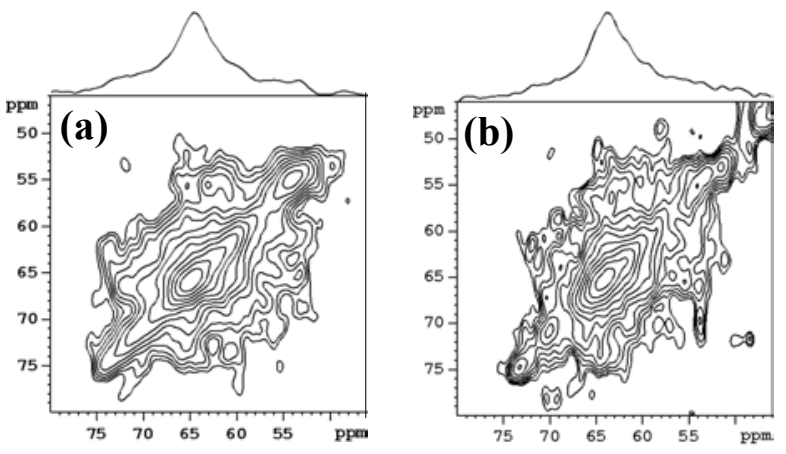

Figure 5-2 2D exchange spectrum at $208 \mathrm{~K}$ of (a) methylene carbons in pure PIB with a $2 \mathrm{~s}$ mixing time; (b) methylene carbons of PIB in a 50:50 blend with PEB using a $100 \mathrm{~ms}$ mixing time. Slices through the 65-ppm trans-gauche peak are shown on the top axis, and within the signal-to-noise limits, exhibit the same features.

butene (PEB), respectively. The PEB contained 49 mole\% 1-butene units randomly incorporated into the chain. The $2 \mathrm{D}$ exchange patterns appear almost identical in terms of their off-diagonal intensity distribution, as confirmed by 
taking a slice through the tg peak at $63 \mathrm{ppm}$ in each. This slice is shown as the top trace on each contour plot. However, while the PIB exchange pattern required a 2-second mixing time to achieve the off-diagonal signal in 5-2(a), the PIB/PEB blend pattern in 5-2(b) was obtained after only 100 milliseconds. A major question in the study of polyolefin blend miscibility is the identification of the thermodynamic driving force for mixing, and in this case, PIB/PEB blends have been shown to be miscible for the PEB composition used here. ${ }^{11,15}$ The results of Figure 5-2 suggest that the rate of conformational dynamics of the PIB chains in the blend with PEB are enhanced, or stated differently, the PIB "local $\mathrm{T}_{\mathrm{g}}$ " is depressed in the blend. The reduced exchange time (a kinetic quantity) of the PIB chains in the PIB/PEB blend may be related to the increased configurational entropy $S_{c}$ (a thermodynamic quantity) using Adams-Gibbs theory, ${ }^{19,}{ }^{124}$ one form of which is shown below:

$$
\tau_{e x}=\tau_{o} \exp \left(\frac{c}{T S_{C}}\right)
$$

Here, $\tau_{\mathrm{ex}}$ is the exchange time for the redistribution of a single conformer's magnetization among all possible conformers, as measured by the $2 \mathrm{D}$ slices shown in Figure 5-2. This exchange time is analogous to a structural relaxation time, and represents the extent of conformational relaxation along the chain. Using the data from Figure 5-2, in which $\tau_{\mathrm{ex}}=2 \mathrm{~s}$ and $100 \mathrm{~ms}$ for pure PIB and PIB in the PIB/PEB blend, respectively, we can solve for the configurational 
entropy ratio $\mathbf{S}_{\text {blend }}: \mathbf{S}_{\text {pure }}$. In this way, the constants $\tau_{\mathrm{o}}$ and $\mathrm{c}$, and the temperature $\mathrm{T}$, cancel from the equation. The resulting $\mathbf{S}_{\text {blend }}: \mathbf{S}_{\text {pure }} \geq 3.33$, indicating that the PIB chains in the blend with PEB have a much larger configurational entropy, and therefore, more accessible conformations per unit time, than pure PIB. Similar results were obtained by comparing equivalent $2 \mathrm{D}$ slices for $\mathrm{PIB}$ and $\mathrm{PIB} / \mathrm{PEB}$ at $4 \mathrm{~s}$ and $1.5 \mathrm{~s}$ mix times, respectively, at the same temperature as Figure 5-2. While this data is not shown for brevity, a similar $\mathbf{S}_{\text {blend }}: \mathbf{S}_{\text {pure }} \geq 3.4$ result was obtained.

\section{5-4 Conclusions}

These results suggest that entropy drives miscibility in this particular polyolefin blend, and the ability to place quantitative limits on key thermodynamic parameters should prove critical to a complete understanding of the heretofore-anomalous results in the area of polyolefin miscibility. Further, Adams-Gibbs theory requires that this increased entropy in the blend corresponds to smaller-sized cooperatively rearranging regions at a given temperature, i.e., a shorter $\mathrm{T}_{\mathrm{g}}$ length-scale. ${ }^{116-118}$ Local motions become dominant in this regime, and the $2 \mathrm{D}$ exchange is clearly sensitive to these subtle changes. An alternative experimental strategy is to match the necessary equivalent $2 \mathrm{D}$ exchange patterns vs. $\mathrm{T}$, thereby obtaining thermally activated configurational entropy changes in any amorphous polymer for which conformationally inequivalent signals are present. We plan to pursue additional experiments as a route to understanding the 
glass-transition length scale and polymer miscibility. 


\section{Chapter 6: Length Scales Which Perturb Chain Packing in Amorphous Polymers}

Marcin Wachowicz, ${ }^{\ddagger}$ Justyna Wolak, Hanna Gracz, Edward O. Stejskal, Stefan

$$
\text { Jurga, }
$$

Elizabeth F. McCord, ${ }^{\circ}$ and Jeffery L. White*

Department of Chemistry, Campus Box 8204

North Carolina State University, Raleigh, NC 27695-8204

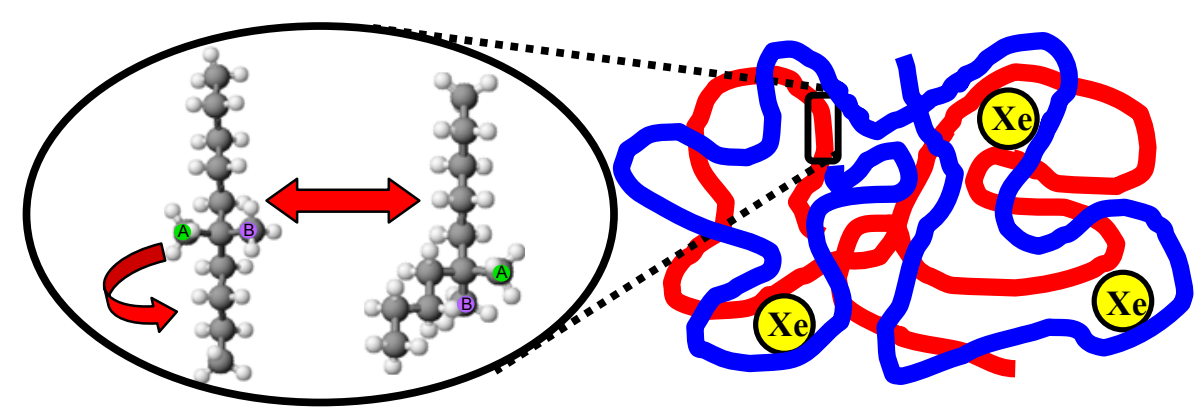

As published in Macromolecules, 2004, Vol.37, p.4573-4579 


\section{6-1 Abstract}

Direct spectroscopic probes of individual chain conformation and free volume are used to measure the increasing perturbation in the local glasstransition temperature of one polymer chain with decreasing length scale of mixing in binary polyolefin blends. Solid-state ${ }^{2} \mathrm{H}$ and ${ }^{129} \mathrm{Xe}$ NMR experiments reveal a compositional miscibility window in side-chain concentration for polyisobutylene (PIB)/polyethylene-co-butene (PEB) blends. A combination of pulsed-field gradient and chemical shift data for xenon gas absorbed in these polymer blends indicates that the presence of polymer chains within a radius of 35 $\mathrm{nm}$ of a different chain structure will perturb the intermolecular packing contribution to the total conformational energy of that chain, thereby changing its $\mathrm{T}_{\mathrm{g}}$

₹ Department of Macromolecular Physics, Institute of Physics, Adam Mickiewicz University, ul. Umultowska 85, 61-614 Poznan, Poland.

○ DuPont Central Research and Development, Experimental Station, Wilmington, Delaware

\section{6-2 Introduction}

Kumar and coworkers have recently published a theoretical paper in which they question the length scales that control dynamics in miscible polymer blends specifically, and polymer chains in general. ${ }^{125}$ Key considerations in that work include the influence of concentration fluctuations, chain connectivity effects, the size of dynamic heterogeneities or cooperatively rearranging regions, and their temperature dependencies. As evidenced by the large number of recent publications in this general area, these topics are relevant to structure-property 
relationships in both polymers and inorganic glasses, ${ }^{116,126-128}$ the glass transition length scale, ${ }^{117,} 119,{ }^{129-132}$ the glass transition time scale, ${ }^{18}$ and structure relaxation. ${ }^{118,123,133}$ While much of the recent published work has attempted to discern the size- or length-scale of cooperative motions giving rise to the glass transition, we feel an equally important question for blends of amorphous molecules has to do with what length scale of mixing in a binary mixture is required to change the glass transition characteristics (temperature or time scale) of either component relative to their pure states. Stated differently, one could ask what minimum length scale of concentration fluctuation (or domain size) is required to render all molecules in that region "dynamically perturbed" relative to those same molecules in their pure, bulk environment. The ramifications of this issue are clear for the physical properties of binary polymer blends, but are also relevant to the general behavior of polymer interfaces, surfaces, and thin films.

In this contribution, we address the question of length scales experimentally using local spectroscopic probes of polymer chain dynamics and free volume in binary blends of non-associating polyolefins. Solid-state NMR experiments reveal that different perturbations of the chain-level $T_{g}$ take place in blends of polyisobutylene (PIB) and polyethylene-co-1-butene (PEB), in which the 1-butene comonomer concentration is varied among the blends. Specifically, we monitor the change in local chain dynamics of PIB as the length scale of mixing with PEB changes in the blends. A combination of static ${ }^{2} \mathrm{H}$ (a noninvasive, direct probe of polymer chain dynamics) and ${ }^{129} \mathrm{Xe}$ (a non-invasive, direct probe of local chain packing) NMR experiments, as well as ${ }^{129} \mathrm{Xe}$ pulsed- 
field gradient diffusion (PFG) NMR, in these solid polymer blends reveals that concentration fluctuations of $60-70 \mathrm{~nm}$ (several times $\mathrm{R}_{\mathrm{g}}$ ) lead to measurable changes in the dynamics of chains included in those regions, relative to their pure bulk dynamics.

\section{6-3 Experimental}

Copolymers of hydrogenated/perdeuterated polyisobutylene were prepared by cationic polymerization of isobutylene monomers. Copolymers of perdeuterated monomers and their hydrogenated analogues are referenced as follows: $80 \%-P I B-d_{8}\left(M_{n}=466,000\right)$ copolymer denotes $80 \%$ perdeuterated monomers, and 20\%-PIB- $\mathrm{d}_{8} \quad\left(\mathrm{M}_{\mathrm{n}}=353,000\right)$ copolymer indicates $20 \%$ perdeuteration. Commercial PIB $\left(\mathrm{M}_{\mathrm{w}}=1,000,000\right)$ obtained from ExxonMobil Chemical was used for the xenon experiments. The PEB-66 $\left(\mathrm{M}_{\mathrm{w}}=114,000\right)$ is the same polymer previously referenced as HPB66 by Graessley and coworkers, and is a monodisperse ethylene-butene copolymer obtained by anionic polymerization

of butadiene, followed by hydrogenation. ${ }^{11,14,15}$ The degree to which the diene polymerizes 1,2 vs. 1,4 addition determines the butene and ethylene concentrations, respectively, as has been extensively discussed in previous papers. ${ }^{31}$ The PEB-23 sample is a commerical ethylene-butene copolymer made via metallocene polymerization $\left(\mathrm{M}_{\mathrm{w}}=79,000\right)$, and sold as Exact 4041 by ExxonMobil. The 50:50 weight $\%$ blends were prepared from dissolution in toluene for 24-48 hours, followed by solvent evaporation, and then vacuum drying to $10^{-2}$ torr for 4 days or longer. Thermal analysis of each polymer by DSC 
revealed the following $\mathrm{T}_{\mathrm{g}}$ 's: $\mathrm{PIB}=-68{ }^{\circ} \mathrm{C}$; PEB-66 $=-52{ }^{\circ} \mathrm{C}$; PEB-23 $=-39{ }^{\circ} \mathrm{C}$; PIB/PEB-66 blend = broad, poorly resolved transition at ca. $-61{ }^{\circ} \mathrm{C}$; PIB/PEB-23 blend $=$ two well-resolved transitions at $-68^{\circ} \mathrm{C}$ and $-39^{\circ} \mathrm{C}$.

All ${ }^{2} \mathrm{H}-\mathrm{NMR}$ measurements were performed on a Bruker CXP spectrometer operating at a magnetic field strength of $4.7 \mathrm{~T}$, corresponding to 30.7 $\mathrm{MHz}$ deuteron resonance frequency. The spectra were recorded using the quadrupole-echo pulse sequence $\left((\pi / 2)_{\mathrm{x}}-\tau_{1}-(\pi / 2)_{\mathrm{y}}-\tau_{2}-\right.$ acq) with a typical pulse width of $3.0 \mu$ s. Consequently, pure perdeuterated PIB spectra required 512 scans while the blends required up to 4000 scans to obtain spectra of sufficient sensitivity. The temperature dependence of $\mathrm{T}_{2}{ }^{\mathrm{QE}}$ and $\mathrm{T}_{1}$ relaxation times was measured using the quadrupolar echo (as mentioned above) and inversionrecovery with quadrupole echo detection $\left((\pi)_{x}-\tau_{1}-(\pi / 2)_{x}-\tau-(\pi / 2)_{y}-\tau-\right.$ acq) sequences, respectively.

Weblab version 4.1, an interactive web-based program, was used for deuteron line shape simulations of the 4-site exchange model. ${ }^{134}$ The program can be used to calculate quadrupolar echo spectra of $\mathrm{I}=1$ spin system under the influence of molecular motions. Weblab offers ${ }^{2} \mathrm{H}-\mathrm{NMR}$ one-dimensional theoretical spectrum calculations but it is limited to discrete jumps on a single cone or the n-state jump model. The simulation input parameters consisted of angles, site populations, and reorientation rates while considering two types of motion including fast methyl rotation and trans-gauche isomerization. Simulations were repeated until satisfactory correlation was accomplished between the experimental and the simulated spectra. 
${ }^{129} \mathrm{Xe}$ single-pulse NMR experiments were performed on a Bruker AVANCE $500 \mathrm{MHz}$ spectrometer with an Oxford narrow bore magnet. A 10-mm probe (BB500-10EB, Nalorac Cryogenic Corp.) was used for all measurements and all spectra were acquired at $298 \mathrm{~K}$ (with the line from free xenon gas serving as the internal standard at $0 \mathrm{ppm}$ ). The instrumental parameters for acquisition of the ${ }^{129} \mathrm{Xe}$ NMR spectra are as follows: spectrometer frequency $=138.365 \mathrm{MHz}$; spectral width $=83333 \mathrm{~Hz}$ (equivalent to $602 \mathrm{ppm}$ ); number of data points $=16$ $\mathrm{K}$; relaxation delay $=2 \mathrm{~s}$; acquisition time $=0.098 \mathrm{~s}$; pulse width $=16 \mu$ s (tip angle $=60^{\circ}$ ); number of transients $>1 \mathrm{~K}$. A thick-walled, valved NMR tube from New Era Enterprises (Part \# NE-CAV5-M-133) was used in conjuction with a Swagelok adapter (Part\# 2-TA-1-OR) to transfer gas from a pressurized line (1/4 inch stainless steel tubing) to the sample. The tube allows for pressure-controlled experiments by its precise Teflon plug with a Viton O-ring seal. A delrin adapter was made to attach the NMR tube to the xenon line. In order to ensure that no pressure blow-out would occur and in order to give more physical control over the sealing method, a Teflon connection was made and connected the sleeve of the NMR tube to the adapter. This sample preparation arrangement is attractive relative to previously published custom designs, since all components may be obtained commercially. Typically 120 psig (equivalent to 9.3 bar, total pressure) of xenon was used; equilibration was almost instantaneous.

The ${ }^{129}$ Xe diffusion NMR results were obtained on a Varian $400 \mathrm{MHz}$ Inova wide bore NMR spectrometer with a high gradient strength (up to 1000 $\mathrm{G} / \mathrm{cm}$ ) variable temperature double-coil DOTY diffusion probe equipped with 
water-cooled gradients. Experiments were run at $30.2{ }^{\circ} \mathrm{C}$ using air-cooling. Temperature was calibrated using a thermocouple centered axially in a $5 \mathrm{~mm}$ NMR tube at the center of the receiver coil position under normal air-flow conditions. The standard Varian pulse sequence was modified to use trapezoidal shaped diffusion gradient pulses. The console was equipped with a Performa 3 amplifier whose output was connected to a Techron high power amplifier for the gradients. Experiments were run using acquisition times of $0.64 \mathrm{sec}$, a recycle delay time of 3 or $5 \mathrm{sec}$, a $14.75 \mu \mathrm{sec} 90^{\circ}$ pulse, a spectral width of $38.7 \mathrm{kHz}, 800$ or more transients averaged per increment, analog filters, and 8 increments with diffusion gradients ranging from $50-950 \mathrm{~g} / \mathrm{cm}$. The optimal gradient pulse length was $4 \mathrm{~ms}$ and the diffusion delay was $50 \mathrm{~ms}$; no differences were observed using a 20-ms delay. Data were processed using line broadening of $40 \mathrm{~Hz}$ and zero filling to $128 \mathrm{~K}$. We used a mono-exponential fit to analyze the data with a weighting function of the inverse square root of the peak intensity. Our data processing force fits to a single exponential even if the species exists in multiple environments and the raw data is a sum of several different exponential decays.

\section{6-4 Results and Discussion}

\section{${ }^{2}$ H NMR Data}

In Figure 6-1, a stack plot of ${ }^{2} \mathrm{H}$ NMR quadrupole echo spectra of $80 \%$ PIB- $\mathrm{d}_{8}$ are shown as a function of temperature, ranging from $210 \mathrm{~K}$ to $294 \mathrm{~K}$. At the lowest temperature, Figure 6-1 shows an overlapping Pake pattern spectrum composed of $\mathrm{CD}_{2}$ and $\mathrm{CD}_{3}$ signals. The signals can be assigned to $\mathrm{CD}_{2}$ and $\mathrm{CD}_{3}$ 


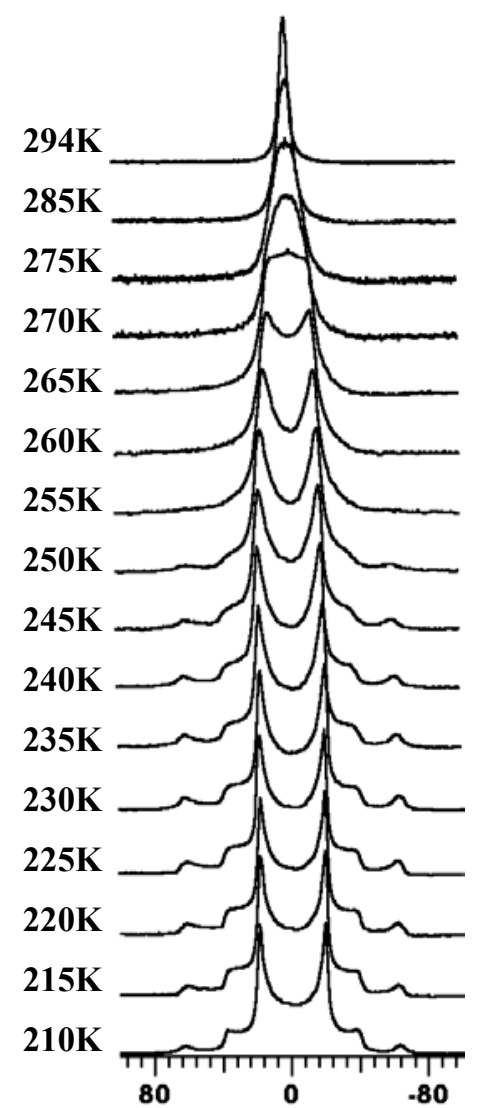

Figure 6-1 Static ${ }^{2} \mathrm{H}$ NMR spectra for $80 \%$-PIB- $\mathrm{d}_{8}$ versus temperature, showing a glass transition on the ${ }^{2} \mathrm{H}$ NMR timescale at $\approx 265 \mathrm{~K}$.

by relative amplitudes (2/8 and 6/8) and different quadrupolar splittings $(128 \mathrm{kHz}$ and $39 \mathrm{kHz}$ ), respectively. ${ }^{38}$ Due to the large $128 \mathrm{kHz}$ splitting, the $\mathrm{CD}_{2}$ signal collapses beneath the $\mathrm{CD}_{3}$ signal at higher temperatures and becomes observable only indirectly. At $210 \mathrm{~K}$, the $\mathrm{CD}_{3}$ group has an anisotropically averaged Pake pattern with a $39 \mathrm{kHz}$ splitting, due to rapid rotation along $\mathrm{C}_{3}$ at a rate greater than the quadrupolar coupling interaction. In order for additional narrowing of the $\mathrm{CD}_{3}$ signal to take place above $210 \mathrm{~K}$, an additional source of motion that reorients the bond vector between the methyl and the quaternary carbon is required. Since the 
$\mathrm{CD}_{3}$ group is directly attached to the quaternary carbon, it acts as a specific probe of the chain backbone conformational dynamics. Further narrowing takes place with increasing temperature due to the slower reorientations of the chain via transgauche conformational isomerization. The Pake pattern collapses into a single line with no splitting at $265 \mathrm{~K}$. This coalescence point is followed by further reduction in the linewidth, to yield a simple Lorentzian lineshape at room temperature.

Figure 6-2 illustrates the unique lineshape coalescence point for the three samples versus temperature. The pure $20 \%$-PIB- $\mathrm{d}_{8}$ sample coalesces at $265 \mathrm{~K}$ (Figure 6-2(c)), while PIB/PEB-66 (Figure 6-2(a)) and PIB/PEB-23 (Figure 62(b)) coalesce at $255 \mathrm{~K}$ and $260 \mathrm{~K}$, respectively. The variations in $\mathrm{CD}_{3}$ line shape for the PIB chains in the respective samples reflect the different frequencies of PIB chain reorientation in each environment. The Pake pattern for the PIB/PEB66 blend exhibits an NMR glass transition at 255K while PIB/PEB-23 blend does so at $260 \mathrm{~K}$. Clearly, the PIB chain motional frequencies are perturbed more in the PIB/PEB-66 blend (containing 49\% mol butene) than in the PIB/PEB-23 blend (containing 13\% butene). Further inspection of the linewidth and line shape at $265 \mathrm{~K}$, the pure $20 \%-\mathrm{PIB}-\mathrm{d}_{8}$ coalescence temperature, indicates that the PIB/PEB66 lineshape is already much more averaged than that of PIB/PEB-23. Clearly, PIB chains in both blends exhibit increased conformational mobility relative to the bulk PIB, as a result changing the particular lineshape coalescence points of PIB/PEB-23 and PIB/PEB-66 by -5 and -8 degrees, respectively. We were particularly surprised to see the $-5 \mathrm{~K}$ shift for the PIB/PEB-23 blend, since the DSC thermogram shows two distinct, well-resolved $\mathrm{T}_{\mathrm{g}}$ 's (not shown). 


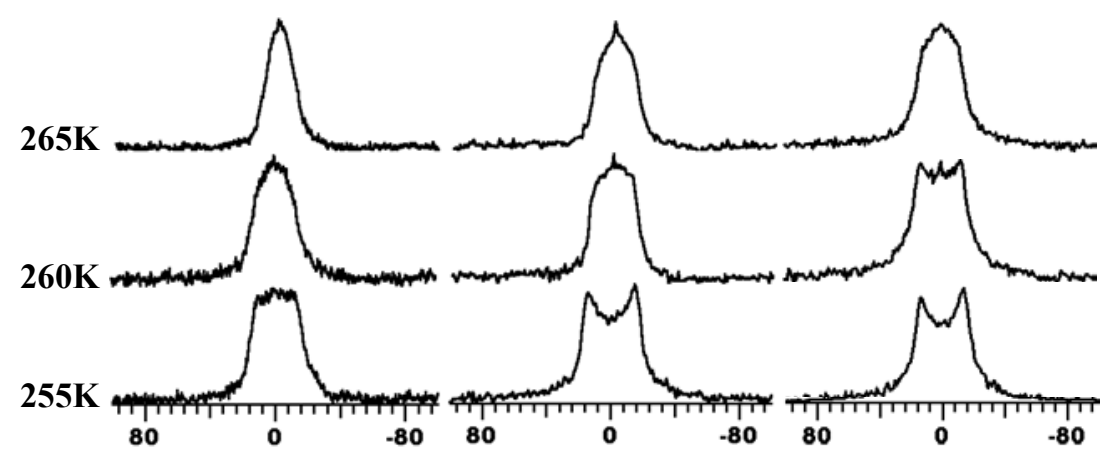

(a)

(b)

(c)

Figure 6-2 Comparison of static ${ }^{2} \mathrm{H}$ NMR spectra over the NMR $\mathrm{T}_{\mathrm{g}}$ range for $20 \%-\mathrm{PIB}-\mathrm{d}_{8}$ chains in (a) a blend with PEB-66; (b) a blend with PEB23; (c) bulk, i.e., pure 20\%-PIB-d $\mathrm{d}_{8}$. Note the difference in the spectral coalescence points in both (a) and (b), relative to (c), which is even more clear by comparing the lineshapes across the second row.

\section{${ }^{2} \mathrm{H}$ Lineshape Simulations}

Deuterium spectra simulations were used to extract models of the PIB chain dynamics in the pure PIB and in the two blends. Reasons for restricting the simulations to the $\mathrm{CD}_{3}$ group and excluding the $\mathrm{CD}_{2}$ group will be discussed later. Using the simulation program Weblab, ${ }^{134}$ two main types of molecular reorientations were included in the $\mathrm{CD}_{3}$ spectra simulations: fast methyl group rotation and trans-gauche backbone isomerization. Fast methyl rotation reduces the quadrupolar splitting by a factor of 3 while the slower trans-gauche chain isomerization leads to further averaging, and finally, to the collapse of the quadrupolar splitting. The trans-gauche isomerization is frozen at temperatures below $\sim 230 \mathrm{~K}$, whereas the methyl rotation is still fast on that timescale. This is confirmed by the low temperature spectra in Figure 6-1 and 6-2, which exhibit 
the motionally narrowed splitting of $\sim 39 \mathrm{kHz} .{ }^{38}$ The value is slightly smaller than the $42 \mathrm{kHz}$ theoretical value. This splitting is reduced by a factor of 3 with respect to the value observed for the spectrum of static methyl groups. In the case of methylene groups, once the chain trans-gauche isomerization is frozen there are no other mechanisms that could average the quadrupolar interaction and consequently a static powder type spectrum of $128 \mathrm{kHz}$ is observed.

Different n-site jump models were considered for the trans-gauche isomerization. Ultimately, a four-site exchange model was chosen, with the flip angle equal to $90^{\circ}$ and the cone angle equal to the 'magic angle' $\theta=54.74^{\circ}$. The possible jumps in the 4-site exchange model are based on the jumps described by the 3-site model (flip angle $\Phi=120^{\circ}$, cone angle $\Theta=70.5^{\circ}$ ), except they are observed from a coordinate system that is tilted by an angle of $54.74^{\circ}$. Figure 6-3 illustrates two chain segments used for the simulations, showing the all-trans (a) with two methyl groups marked as 'A' and 'B' switching to a gauche (+ or -) (b) conformation on the top left and right, respectively. The bottom schematic denotes the conformational dynamics model used in the motionally-averaged $\mathrm{CD}_{3}$ powder simulations, with the circle showing the different sites occupied with either methylene or methyl groups in the trans (a) and gauche (b) states. The simulation input parameters included the angles (defining the site orientation involved in a motion), site populations (occupancy probabilities), and reorientation rates (exchange rates among sites). The site probabilities were defined as follows: $\mathrm{p}_{1}$ for $\Phi=-135^{\circ}, \mathrm{p}_{2}$ for $\Phi=-45^{\circ}, \mathrm{p}_{3}$ for $\Phi=45^{\circ}, \mathrm{p}_{4}$ for $\Phi=$ $135^{\circ}$. These probabilities determine the exchange rates among different sites, 

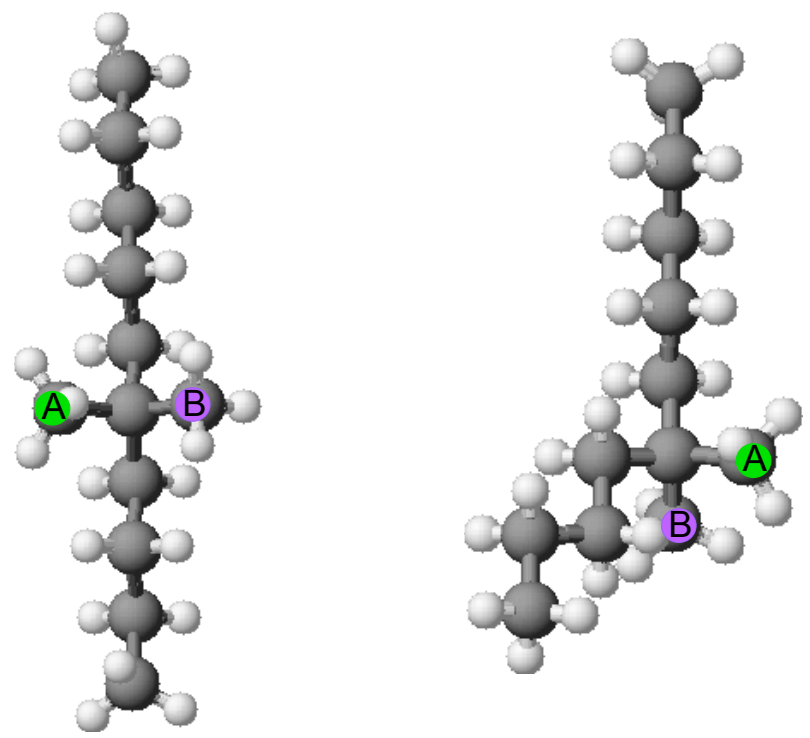

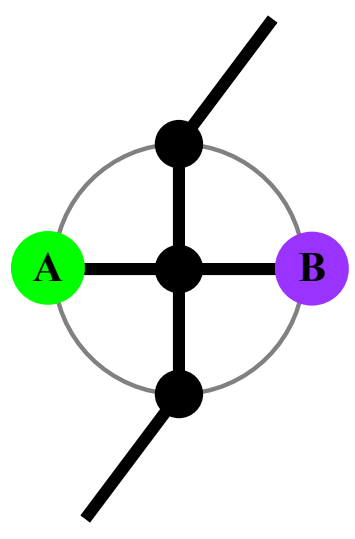

(a)

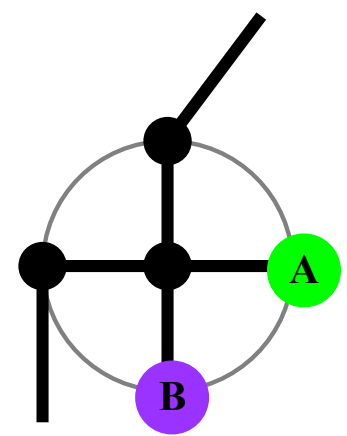

(b)

Figure 6-3 Top schematic shows a simulated conformer of an (a) all trans chain segment with introduction of a single trans-gauche (gauche + or gauche -) exchange event in (b). Bottom schematic shows conformational dynamics model used to simulate the motionally-averaged $\mathrm{CD}_{3}$ powder patterns. Individual methyl groups are denoted as " $\mathrm{A}$ " and "B".

which are contained in the exchange matrix. A limitation of the Weblab model is that the exchange rates between sites ' 1 ' and ' 3 ' (trans $\rightarrow$ trans), as well as between ' 2 ' and '4' (gauche+ $\rightarrow$ gauche-), were zero $\left(v_{13}=v_{31}=v_{24}=v_{42}=0\right)$. The sum of site probabilities $\mathrm{p}_{1}+\mathrm{p}_{3}$ corresponded to the total trans conformation 
probability, while $\mathrm{p}_{2}+\mathrm{p}_{4}$ corresponded to the total gauche conformation probability. An increased 'gauche' site probability results from higher frequency of trans $\rightarrow$ gauche jumps and similarly the increased trans probability results from the fact that gauche $\rightarrow$ trans reorientation dominates over the inverse process. Simulations showed the expected increase in gauche conformer population with increasing temperature (not shown).

Theoretical spectra were calculated starting with the room temperature experimental line shapes, and then varying the relative trans and gauche conformational contributions together with the reorientation rates. Figure 6-4 demonstrates the excellent agreement between the experimental (Figure 6-4(a)) and simulated (Figure 6-4(b)) spectra for PIB/PEB-23. In order to gain correlation at lower temperatures, the average reorientation rate was decreased and site probabilities were systematically changed causing the expected decrease of gauche chain conformational contributions. Equal probabilities for gauche ${ }^{+}$and gauche ${ }^{-}$conformations were assumed $\left(\mathrm{p}_{2}=\mathrm{p}_{4}\right)$. The 4 -site model has two sites that correspond to the trans conformation $\left(\mathrm{p}_{1}, \mathrm{p}_{3}\right)$ by which one can achieve a jump rate distribution that will vanish in the limit of $\mathrm{p}_{1}=\mathrm{p}_{3}$ or when one of these probabilities approaches zero.

All three samples (PIB, PIB/PEB-66 and PIB/PEB-23) were accurately simulated using the 4-jump model, which demonstrates that the PIB chain dynamics are the same in all samples. The only difference is that the motional frequency is increased at any temperature in the blends relative to the pure $20 \%$ - 
PIB- $\mathrm{d}_{8}$. As mentioned before, the conformational dynamics of PIB are perturbed in the PIB/PEB-66 blend more than those in PIB/PEB-23, or in other words, the

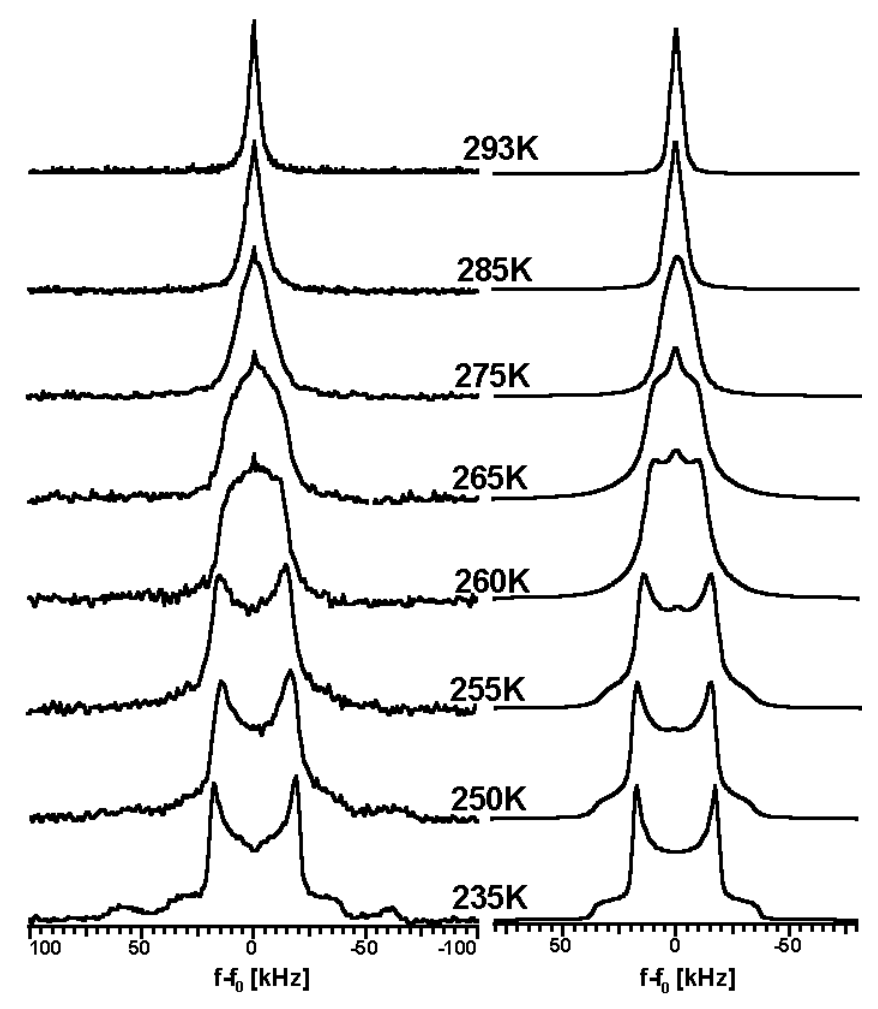

(a)

(b)

Figure 6-4 Comparison of (a) experimental and (b) simulated variable-temperature ${ }^{2} \mathrm{H}$ spectra for the $20 \%$ - PIB- $\mathrm{d}_{8}$ blended with PEB-23.

effective NMR $T_{g}$ is reduced further. Figure 6-5(a) shows the reorientation rate obtained from the 4-site jump model as a function of temperature for the pure 20\%-PIB- $\mathrm{d}_{8}, 20 \%-$ PIB- $\mathrm{d}_{8} /$ PEB-23 and 20\%-PIB- $\mathrm{d}_{8} /$ PEB-66. The slopes of the three systems indicate an isomerization activation energy equal to $36 \mathrm{~kJ} / \mathrm{mol}$. Additionally, the graph signifies intermediate behavior for the PIB/PEB-23 blend relative to the pure $20 \%-\mathrm{PIB}-\mathrm{d}_{8}$ and the PIB/PEB-66. The activation energy obtained from the lineshape simulations can be compared with the model-free 


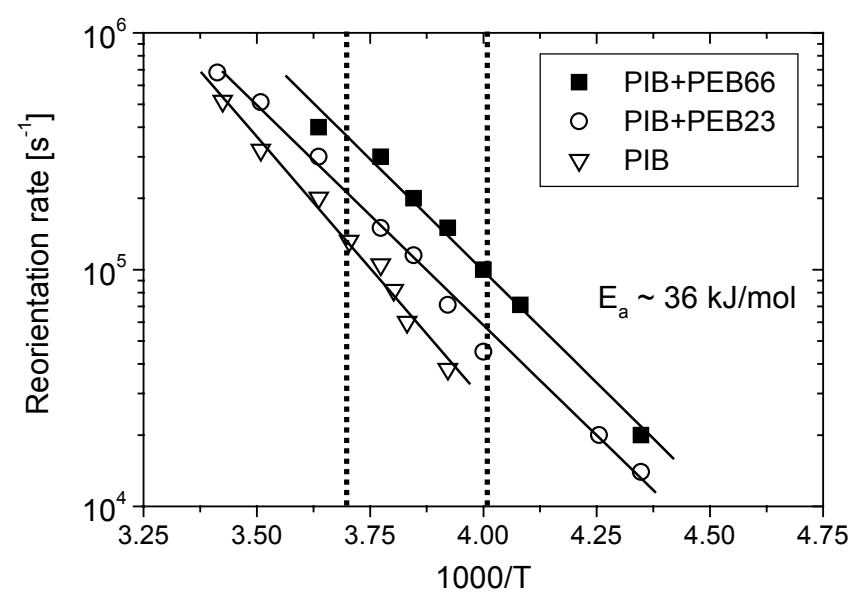

(a)

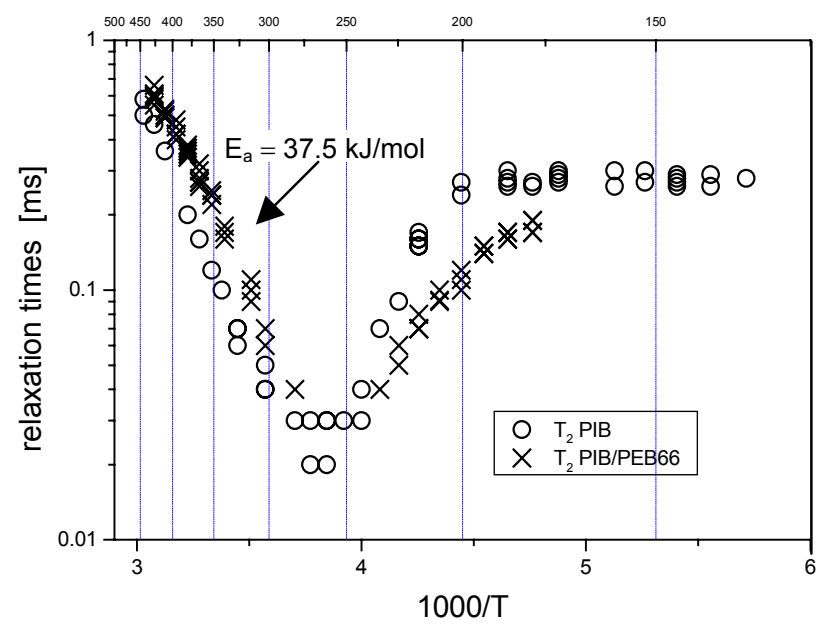

(b)

Figure 6-5 (a) Arrhenius plot of chain reorientation rates obtained via simulation of the experimental static ${ }^{2} \mathrm{H}$ lineshapes using the four-site model described in the text for $20 \%$-PIB-d $\mathrm{d}_{8}$ chains in each of the indicated environments. The dotted lines indicate the region near lineshape collapse. (b) Relaxation rate data vs. temperature for ${ }^{2} \mathrm{H} \mathrm{T}_{2}$ experiments. Note that the same activation energy is obtained via lineshape simulation (a) and in the linear region of the $\mathrm{T}_{2}$ curve (b).

relaxation experiments, e.g. $\mathrm{T}_{2}{ }^{\mathrm{QE}}$ as a function of temperature. From the graph in Figure 6-5(b), the right and upwards shift in the $\mathrm{T}_{2}{ }^{\mathrm{QE}}$ upon blending PIB with PEB-66 is evident, at the same time also revealing an activation energy equal to $37.5 \mathrm{~kJ} / \mathrm{mol}$. The direct link between the simulated and experimental data is 
clearly demonstrated by the matching activation energies acquired by two different methods, one of which is independent of any assumptions or physical model.

Using the direct connection between the simulated and experimental data, the justification for neglecting the $\mathrm{CD}_{2}$ contribution to the theoretical spectrum calculations for the PIB molecule is reasonable. As seen in Figure 6-1 and the PIB/PEB-23 stackplot in Figure 6-4(a), the $\mathrm{CD}_{2}$ contribution was resolved at temperatures below $\sim 230 \mathrm{~K}$ as a broad component with static splitting equal to $\sim 128 \mathrm{kHz}$. Due to the deuteron ratio in the monomer $\left(2 \mathrm{CD}_{3}: \mathrm{CD}_{2}\right.$ or $\left.3: 1\right)$, the integrated intensity of the $\mathrm{CD}_{2}$ group is $\sim 3$ times smaller than the intensity of a $\mathrm{CD}_{3}$ group spectrum. Furthermore, the sensitivity of ${ }^{2} \mathrm{H}-\mathrm{NMR}$ blend spectra was decreased by the lower degree of perdeuterated PIB monomers even with increased acquisitions. The conformational interchange along the backbone will act to narrow the $\mathrm{CD}_{3}$ and $\mathrm{CD}_{2}$ lineshapes at the same rate vs. temperature, i.e., with the same activation energy. Therefore, the relative comparison of activation energies between the three samples should not be complicated by the fact that the $\mathrm{CD}_{2}$ component is not explicitly treated in the simulations.

\section{Basis for Tetrahedral Jump Model Assignment}

In the absence of additional experimental information, one cannot unequivocally assign the observed changes in the ${ }^{2} \mathrm{H}$ lineshapes to $120^{\circ}$ internal rotations associated with rapid tetrahedral jumps, versus small-angle librational motions. ${ }^{135,136}$ English and coworkers have previously discussed the quantitative relationships between motional timescales, echo times, motional models, and the 
one-dimensional ${ }^{2} \mathrm{H}$ lineshape. Based on our previously published twodimensional solid-state ${ }^{13} \mathrm{C}$ exchange experiments for these polymers and their blends, it is clear that individual PIB chain segments are undergoing tetrahedral jumps at a rate of $65-75 \mathrm{kHz}$ over the temperature range associated with lineshape collapse in Figure 6-2. ${ }^{13,18}$ From this work we assigned the jump model to the ${ }^{2} \mathrm{H}$ lineshape simulations, and note that the refocusing efficiency of the integrated lineshape intensity was found to decrease by almost a factor of two when the total echo delay time was increased from 20 to $80 \mu$ s, as expected from reference 23 .

\section{${ }^{129}$ Xe NMR}

${ }^{129} \mathrm{Xe}$ NMR is a sensitive and non-destructive technique capable of probing the length scale of mixing, or domain size, in the two blends. ${ }^{74,} 79$ Xenon's large, highly polarizable electron density, but small van der Waals radius of 2.2 angstroms, makes it an appealing tool for probing slight changes in the electronic environment of blends. ${ }^{137}$ Figure 6-6 demonstrates that simple onepulse Xe NMR experiments are remarkably sensitive to different polyolefin environments. ${ }^{74}$ In Figure 6-6, results are presented for ${ }^{129} \mathrm{Xe}$ chemical shift in pure PIB, pure poly-1-butene (P1B), and several blends. In contrast to all other blends of PIB with PEB or P1B, the PIB/PEB-66 blend (6-6(c)) shows only one resonance, indicating that the xenon molecule is diffusing in a homogenous molecular environment (as defined by the diffusion coefficient of xenon in that blend). For comparison, the PIB/PEB-23 blend shows two clearly resolved resonances $(\mathbf{6}-\mathbf{6}(\mathbf{d}))$, corresponding very closely with the two pure component PIB and PEB-23 shifts. Here, xenon gas diffuses between two dissimilar surroundings, 
which match the two constituents, indicative of a much larger length scale of mixing, or domain size, relative to PIB/PEB-66 (vide infra). A similar microphase-separated result is obtained for a blend of PIB/PEB-97 (6-6(b)). Figures 6-6(b)-(d) reveal a compositional miscibility window in 1-butene concentration for blends of PEB with PIB.

The fact that the chemical homogeneity of a miscible binary blend is accurately reflected by a single Xe signal is illustrated in Figure 6-7 for a blend of PIB with hhPP (head-to-head polypropylene). We would have preferred a direct measurement of mixing via ${ }^{1} \mathrm{H}$ spin-diffusion in the PIB/PEB blends used in this study. ${ }^{107,}{ }^{108}$ Unfortunately, the differences in overall chain dynamics, and ${ }^{1} \mathrm{H}$ chemical shifts in the solid-state, for PIB vs. PEB were insufficient to allow spin-diffusion measurements. Therefore, we did a comparative analysis on a polyolefin blend where both Xe NMR and ${ }^{1} \mathrm{H}$ spin-diffusion experiments were possible. In Figure 6-7, the Xe chemical shift results are compared to ${ }^{1} \mathrm{H}$ spindiffusion NMR data for the same sample of $\mathrm{PIB} / \mathrm{hhPP}$, a blend pair which is known to be miscible. ${ }^{33}$ Indeed, this blend is intimately mixed at the chain level (length scale of $2.5 \mathrm{~nm}$ which is $<<\mathrm{R}_{\mathrm{g}}$ ), as shown by the spin-diffusion plot in Figure 6-7, the analysis of which has been previously published. ${ }^{33,138}$ Figure 6-7 demonstrates that if intimate chain mixing occurs in a polymer blend, a single Xe chemical shift will be observed. However, the presence of a single Xe line does not prove intimate mixing at the chain level; it shows only that the blend is homogeneous on a length scale defined by the Xe diffusion coefficient. 
(a)

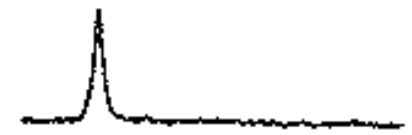

(b)

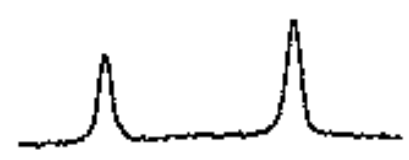

(c)

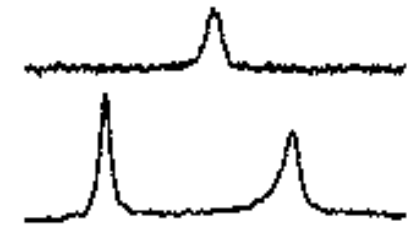

(e)

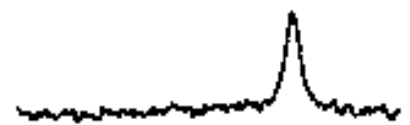

(f)

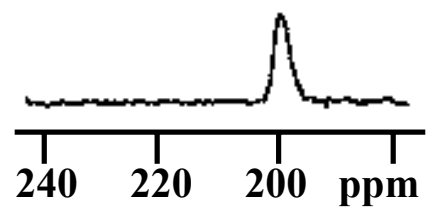

Figure 6-6 Static ${ }^{129} \mathrm{Xe}$ NMR spectra for xenon gas absorbed in (a) pure PIB vs. blends with decreasing 1-butene comonomer amounts: (b) 50:50 PIB/PEB-97 blend; (c) 50:50 PIB/PEB-66 blend; (d) 50:50 PIB/PEB-23 blend; (e) pure PEB-97; (f) pure isotactic poly-1-butene. The free xenon peak at $0 \mathrm{ppm}$ is not shown for clarity, and lower sample masses were used in 6-6(c) and 66(e).

In order to quantitatively analyze the Xe chemical shift data from Figure 6-6 in terms of average domain size or concentration length scale in the PIB/PEB blends, ${ }^{129} \mathrm{Xe}$ pulsed-field gradient (PFG) spin-echo experiments were used to measure the physical diffusion coefficient of Xe in the samples. ${ }^{78,80,139,140}$ In these experiments a magnetic field gradient is used to spatially encode the sample along the z-axis. A delay period is then introduced during which the molecules can diffuse out of place with respect to the $\mathrm{z}$ spatial encoding. Following the delay period, a reverse magnetic field gradient is applied which refocuses the magnetization and produces an echo signal for the molecules which have not diffused out of place. Echo signal measurements as a function of gradient 


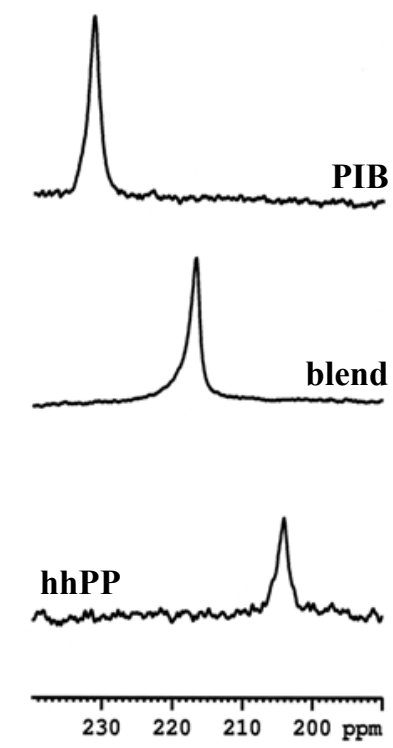

(a)

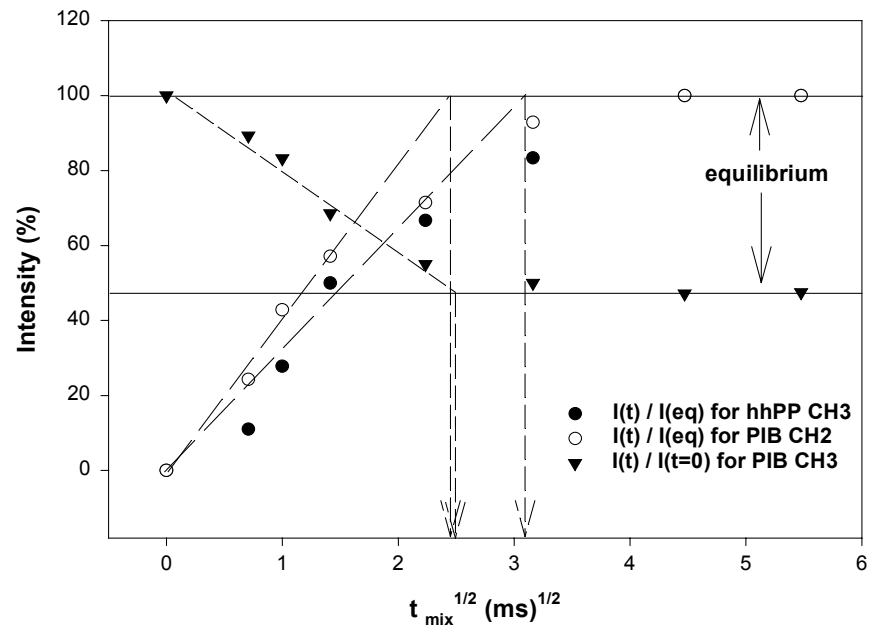

(b)

Figure 6-7 Comparison of two non-invasive experimental methods of miscibility for a polyolefin blend: (a) static ${ }^{129} \mathrm{Xe}$ spectra of a PIB/hhPP blends vs. its pure components; (b) ${ }^{1} \mathrm{H}$ solid-state spin diffusion data for the same blend as in (a). Note that the slopes of all increasing and decreasing curves in (b) are of similar absolute magnitude, indicating that polarization transfer between PIB and hhPP chains occurs with a timescale similar to spin-diffusion within PIB chains, proving intimate mixing (taken from Ref. 33,138).

strength consequently determine the specific diffusion coefficients as seen in Figure 6-8 for PIB and PIB/PEB-66 samples. A least squares analysis of the PFG data according to Equation 6-1 (below) with I (echo intensity), $\gamma$ (magnetogyric rato), $\delta$ (gradient pulse length), G (gradient strength), D (diffusion coefficient), $\tau$ (midpoint separation time of bipolar gradient pulses), and $\Delta$ (diffusion delay) allows the diffusion coefficients for PIB and PIB/PEB-66 to be determined as 2.9 $\times 10^{-8} \mathrm{~cm}^{2} / \mathrm{s}$ and $2.0 \times 10^{-7} \mathrm{~cm}^{2} / \mathrm{s}$, respectively.

$$
I=I_{o} \exp \left[-\gamma^{2} \delta^{2} G^{2} D\left(\Delta-\frac{\delta}{3}-\frac{\tau}{2}\right)\right]
$$




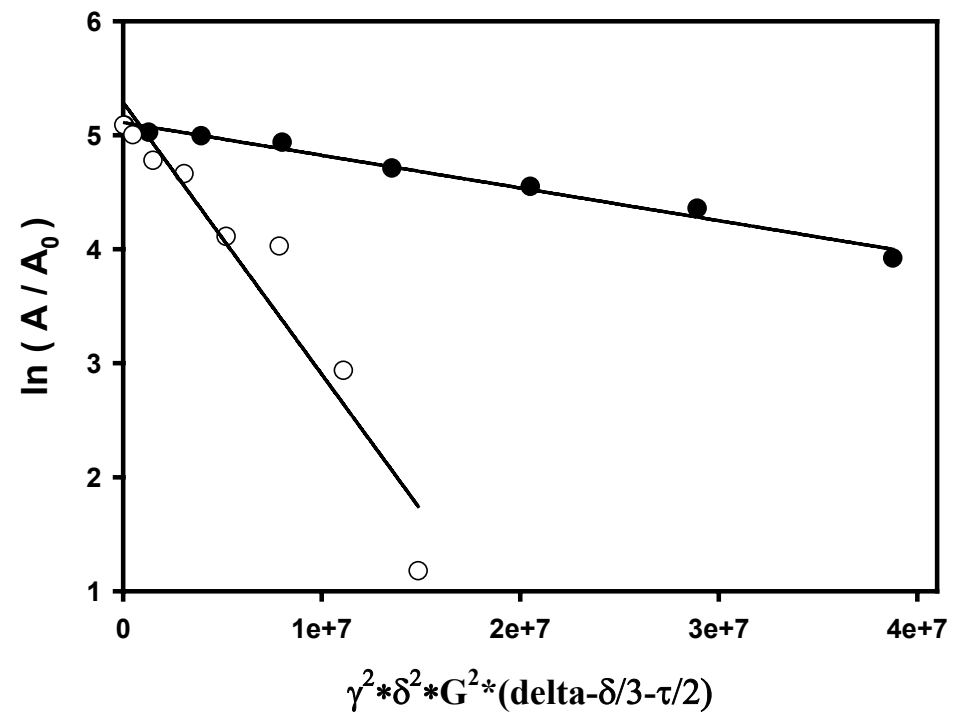

Figure 6-8 ${ }^{129} \mathrm{Xe}$ PFG experimental results for pure PIB (•) and PIB/PEB-66 (o), yielding diffusion coefficients of $2.9 \times 10^{-8} \mathrm{~cm}^{2} / \mathrm{s}$ and $2.0 \times 10^{-7} \mathrm{~cm}^{2} / \mathrm{s}$, respectively.

Almost a factor of seven difference exists for the diffusion coefficients of xenon in pure PIB and PIB/PEB-66, demonstrating that upon blending, the PIB chains adopt a new, less confined packing structure relative to the bulk PIB. Using the

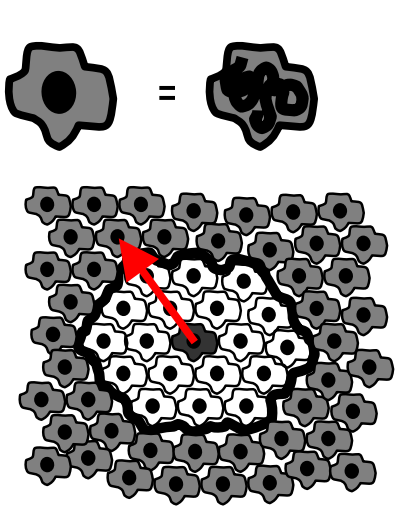

(a)
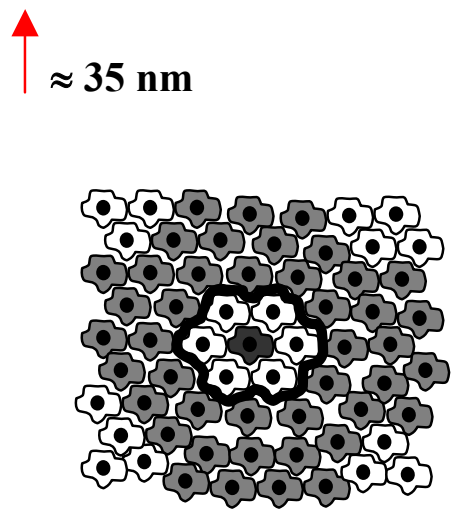

(b)

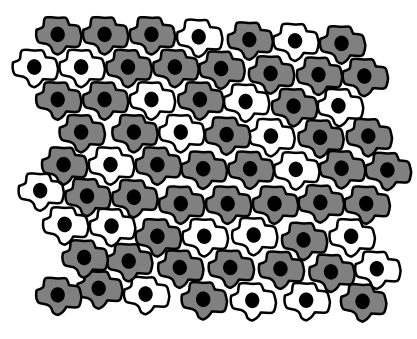

(c)

Figure 6-9 Schematic of mixing length scales derived from ${ }^{129} \mathrm{Xe}$ NMR data in (a) the PIB/PEB23 blend. For the PIB/PEB-66 blend, either (b) or (c) are possible representations, since the average domain size is $<70 \mathrm{~nm}$ for each. 
diffusion coefficient for the PIB/PEB-66 blend together with the chemical shift difference of the two constituents $(4841 \mathrm{~Hz})$, the length scale of mixing in the PIB/PEB-23 blend is calculated to be $>70 \mathrm{~nm}$, while that for the PIB/PEB-66 blend is $<70 \mathrm{~nm}$. Figure 6-9 depicts the organization of polymer chains between PIB-rich and PEB-rich regions.

\section{6-5 Conclusions}

Chain packing in blends of amorphous, non-associating polymers has been shown to be influenced by length scales of mixing even larger than $70 \mathrm{~nm}$, a value which is significantly greater than the dimensions of individual polymer chains in their bulk states $(<10 \mathrm{~nm})$. Conformational dynamics of individual polymer chains at the glass transition are measurably and reproducibly perturbed in blends of polyisobutylene and polyethylene-co-butene that are microphase separated on these length scales, although less so than similar blends with more intimately mixed chains. A miscibility window in 1-butene composition was found for blends of PIB/PEB; blends containing PEB with 49 mole\% 1-butene comonomer were more intimately mixed with PIB than those having 13 or 90 mole\% 1-butene comonomer. Our experiments define an alternative view of the " $\mathrm{T}_{\mathrm{g}}$ length-scale", in that they directly reveal limits on how close one type of polymer chain has to get to another in order to alter its conformational energy surface, i.e., the interchain contribution to its total conformational energy. Our results on binary blends suggest that a gradient of polymer chain dynamics, and $\mathrm{T}_{\mathrm{g}}$ 's, exists at surfaces or interfaces, with the thickness of that gradient equal to several times the polymer radius of gyration. 
CHAPTER 7: CODEX NMR Investigation of Novel Polyolefin Blends 


\section{7-1 Introduction}

The following chapter focuses on a novel system involving atactic polypropylene (aPP), which at the beginning led us to investigate two different sources of the sample. Once the tacticity amount had been established, xenon NMR was applied to several blends of aPP with the previously described polyethylene-co-1-butene (PEB) samples. Miscibility (based on the xenon diffusion coefficient of PIB/PEB-66 blend) was established for aPP/PEB-66. New challenges, such as smaller signal dispersion, prevented the unambiguous application of the $2 \mathrm{D}$ exchange experiment with its restriction of possessing at least one conformationally sensitive signal with a distinct chemical shift.

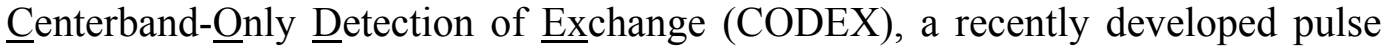
sequence, became a vital tool to simultaneously probe polymer dynamics with maximum spectral resolution and high sensitivity to both blend components.

\section{7-2 Different Sources of Atactic Polypropylene}

Two sources of atactic polypropylene, Eastman Chemical Product Inc. and PolySciences, sparked an investigation into their exact compositions. (We will refer to the Eastman Chemical Product sample as aPP(Eastman) and the PolySciences sample as aPP(PolySciences)). The aPP(Eastman) had a number average molecular weight of 2560 (or 61 repeating units; $\mathrm{DP}=61$ ) while the aPP(PolySciences)'s was between 200,000-500,000. Dissimilar tacticities for the two samples were first detected with the cross-polarization experiment as indicated in Figure 7-1, where the variable temperature studies show different 
cross-polarization results. The methylene regions in Figure 7-1 (a) and (b) display varied populations at the different temperatures. At room temperature, aPP(Eastman) (a) shows three populations that become two main populations at lower and higher temperatures. Conversely, aPP(PolySciences) in (b) has one main population at room temperature, with a second smaller one emerging at temperature extremes as indicated by the dotted lines. Both samples have a slight shoulder around 43ppm indicating a small, third population.

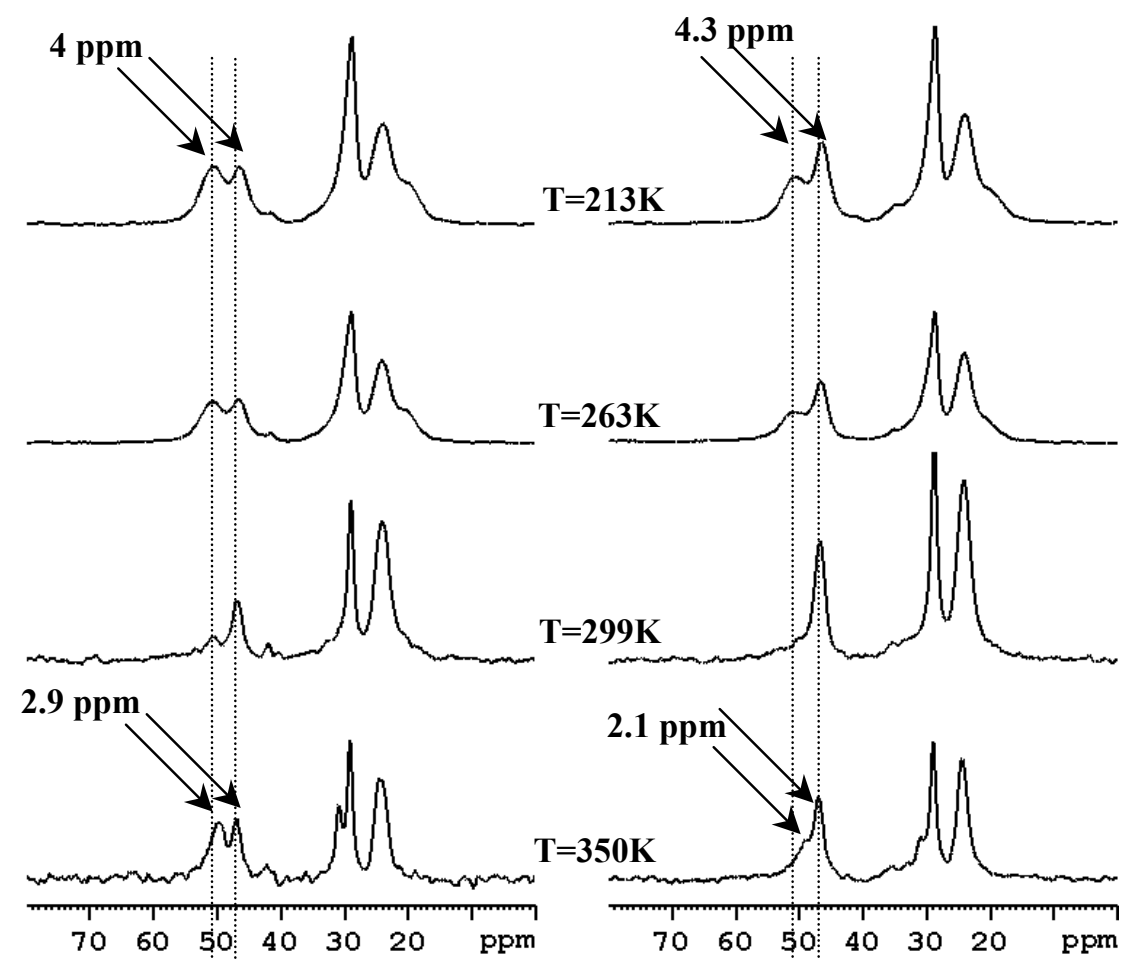

(a)

(b)

Figure 7-1 Variable temperature cross-polarization studies of two samples of (a) aPP(Eastman) and (b) aPP(PolySciences).

In order to determine how much the two samples differed in their tacticities, differential scanning calorimetry plots were pursued to compare the 
differences in their glass transitions. Glass transition will be influenced by differences in tacticities, molecular weight, and the presence of head-to-head and tail-to-tail defects. DSC detected a $\mathrm{T}_{\mathrm{g}}$ of $-10^{\circ} \mathrm{C}$ for aPP (Eastman) and $-15^{\circ} \mathrm{C}$ for aPP(PolySciences).

Tacticity amounts were further investigated using simple solution NMR, where the samples were prepared by being dissolved in 1,1,2,2-tetrachloroethane$d_{2}$ at $360 \mathrm{~K}$, followed by data collection with a simple one-pulse experiment at this

(a)

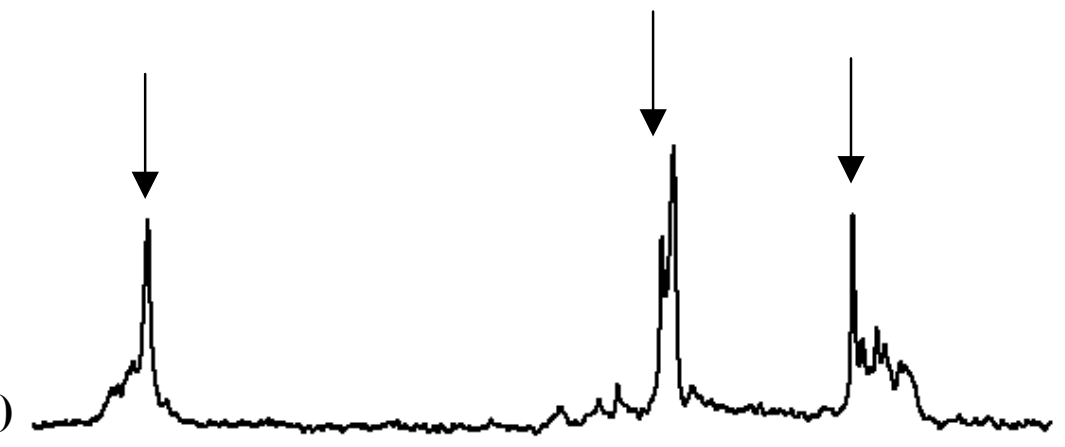

(b)

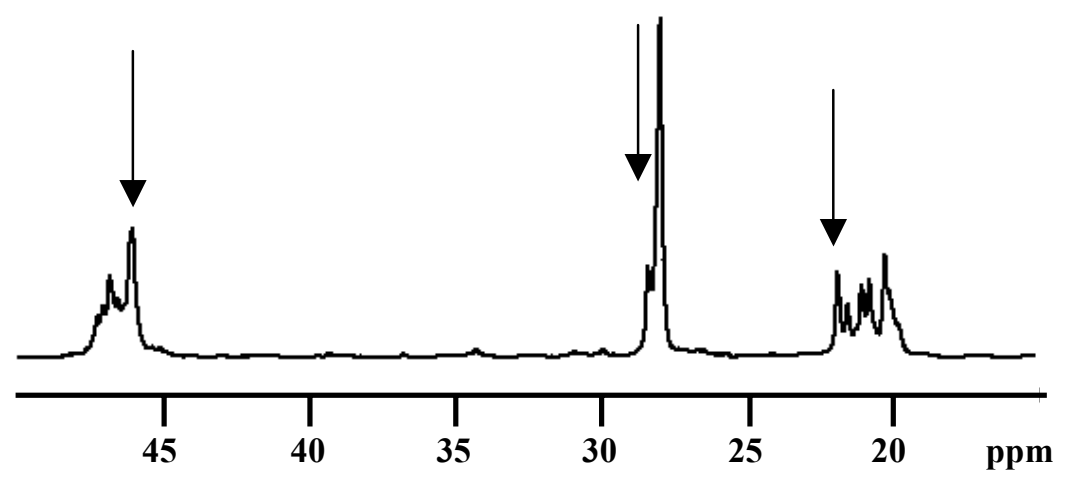

Figure 7-2 Solution spectra taken at $360^{\circ} \mathrm{C}$ for (a) aPP(PolySciences) and (b) aPP(Eastman), which were dissolved in 1,1,2,2-tetrachloroethane- $\mathrm{d}_{2}$.

high temperature. Upon comparing the two solution spectra in Figure 7-2 and recalling Figure 2-1 (a comparison amongst the isotactic, atactic and syndiotactic forms of aPP but dissolved in 1,2,4-trichlorobenzene), features from both 
syndiotactic and isotactic stereosequences can be detected in the atactic samples. The arrows in Figure 7-2 show that the aPP(PolySciences) sample in (a), displays a larger isotactic character as compared with aPP(Eastman) sample shown in (b). The peaks indicated with arrows represent the isotactic form of polypropylene, and the methine and methyl groups in aPP(PolySciences) sample in (a) show the most pronounced isotactic contributions. The combination of the variable temperature cross-polarization studies, DSC plots and solution NMR showed that aPP(Eastman) sample was more atactic, while the aPP(PolySciences) sample had a larger isotactic contribution.

\section{7-3 Miscibility and Dynamics of Atactic Polypropylene Blends}

Following a thorough investigation of the stereosequences present in the two atactic polypropylenes, 50/50 blends with several polyethylene-co-1-butenes were made using the aPP(PolySciences). A rough approximation of the length scales of mixing was established using xenon NMR. As seen in Figure 7-3, there is a much smaller xenon chemical shift difference as compared with the previous system that included PIB (seen in Figure 6-6). The xenon electron density becomes more perturbed with larger polymer densities, and is reflected by a larger chemical shift from free xenon of the dense PIB (232ppm; $\left.\sim 0.91 \mathrm{~g} / \mathrm{cm}^{3}\right)$ versus a smaller shift for aPP $\left(215 \mathrm{ppm} ; \sim 0.85 \mathrm{~g} / \mathrm{cm}^{3}\right) .{ }^{13,76}$ Nevertheless, Figure 7-3 shows that the blend of aPP with PEB-66 is miscible on the length scale defined by the xenon diffusion coefficient. Using the chemical shift difference of its pure components, and the xenon diffusion coefficient from PIB/PEB-66 $\left(\mathrm{D}_{\mathrm{Xe}}=2.0^{*} 10^{-}\right.$ 
$\left.{ }^{7} \mathrm{~cm}^{2} / \mathrm{s}\right),{ }^{141}$ a rough approximation about the length scale of mixing of $100 \mathrm{~nm}$ can be made for this blend. (We will pursue exact xenon diffusion coefficients for

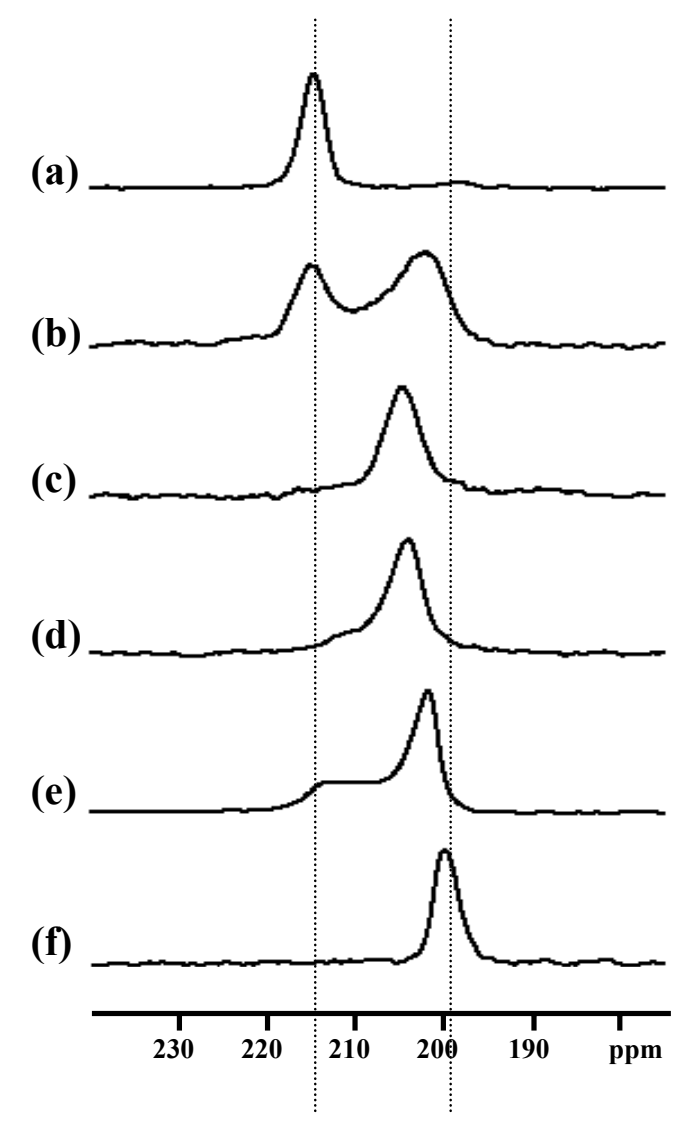

Figure 7-3 Static ${ }^{129} \mathrm{Xe}$ NMR spectra for xenon gas absorbed in (a) pure aPP versus blends with increasing 1-butene comonomer amounts: (b) 50:50 aPP/PEB-23.5; (c) 50:50 aPP/PEB-66; (d) 50:50 aPP/PEB-86; (e) 50:50 aPP/PEB97; (f) pure isotactic poly-1-butene.

these new blends at a later date). This is a large dimension relative to important chain dimensions, but it does differentiate between the blends, defining a lower limit on the length scale of phase separation. All the other blends having 1-butene comonomer concentrations in PEB less than or greater than $66 \mathrm{wt} \%(49 \mathrm{~mol} \%)$ show slow exchange, as indicated by two separate xenon peaks. Regarding the remaining blends in Figure $\mathbf{7 - 3}$, it can be concluded that aPP/PEB-23 in (b) is 
totally immiscible whereas aPP/PEB-86 in (d) and aPP/PEB-97 in (e) are partially miscible due to the closely overlapping peaks.

Furthermore, in order to confirm that the observed lineshape changes seen in Figure 7-3 were due to changes in length scales of mixing, rather than from variations in comonomer concentration in the PEB copolymer, static xenon spectra of all the PEBs utilized were taken. Figure 7-4 shows a xenon stackplot of

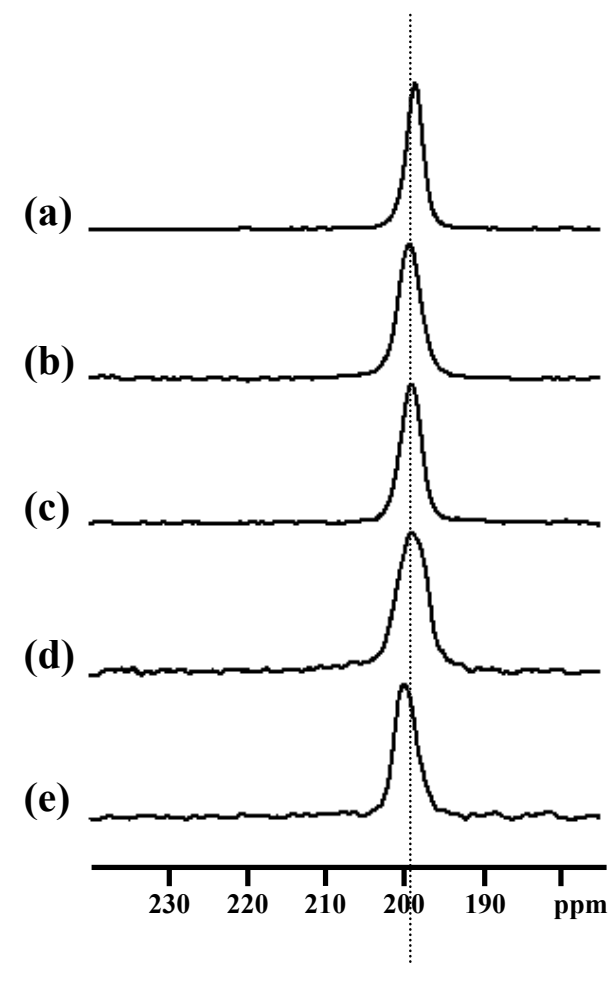

Figure 7-4 Static ${ }^{129} \mathrm{Xe}$ NMR spectra for xenon gas absorbed in PEB copolymers with the following varying 1-butene comonomer concentrations (in weight \%): (a) PEB-23.5; (b) PEB-66; (c) PEB-86; (d) PEB-97; (e) pure isotactic poly-1-butene.

all the pure PEBs, all with extremely similar chemical shifts. Due to the fact that they all had almost exact chemical shifts, it could be concluded that the large 
lineshape changes observed in Figure 7-3, are due to the effects of differential length scales of mixing.

Similar to the xenon signal seen above, the carbon spectra also display much narrower signal dispersion. Figure 7-5 compares a simple low temperature (213K) cross-polarization spectrum of previously studied PIB/PEB-66 (a) versus

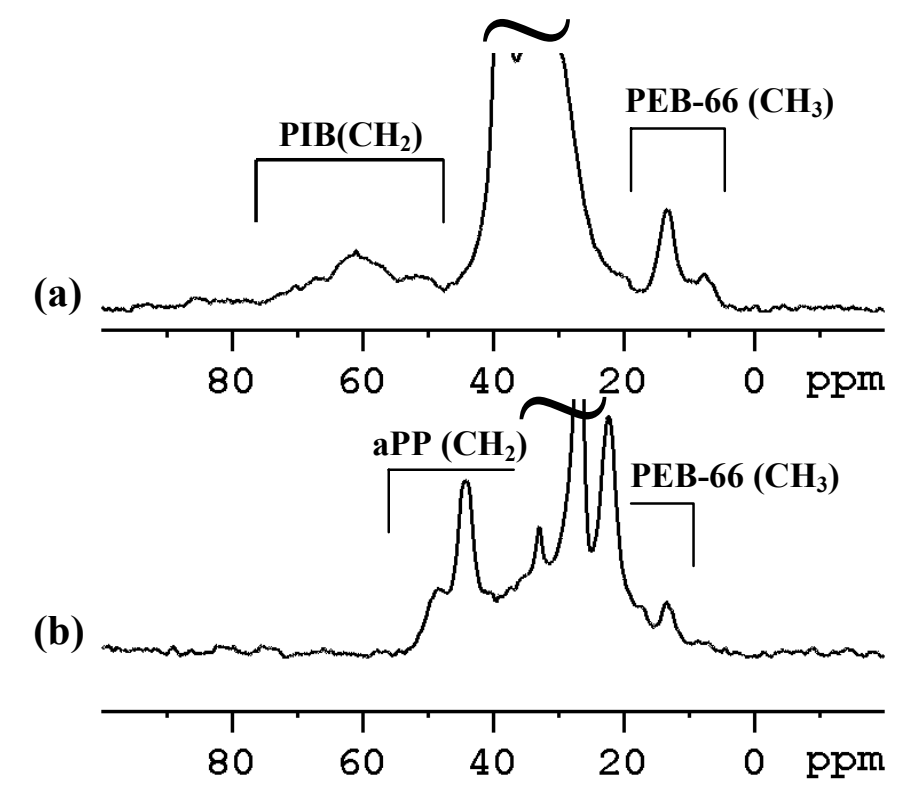

Figure 7-5 A comparison of the chemical shift differences between (a) PIB/PEB-66 versus (b) aPP/PEB-66 via cross-polarization plots performed at $213 \mathrm{~K}$.

that for aPP/PEB-66 (b). As discussed previously, PIB/PEB-66 was a very convenient system in that separate peaks from both components were resolved, facilitating observation of blending effects on both polymers at the same time (in the ${ }^{13} \mathrm{C}$ data). In contrast, as shown in part (b), aPP/PEB-66 has a much narrower signal distribution, with both components severely overlapping with one another. As a result of this peak overlap, the variable temperature cross-polarization stackplot was not very informative. Some major differences between the two 
systems are that PIB is the low $\mathrm{T}_{\mathrm{g}}$ polymer in the PIB/PEB-66 blend $\left(\mathrm{T}_{\mathrm{g}} \mathrm{PIB}\right.$ $=203 \mathrm{~K}$ versus $T_{g}$ PEB-66 $=221 \mathrm{~K}$ ) while $\mathrm{aPP}$ is the higher $\mathrm{T}_{\mathrm{g}}$ polymer in aPP/PEB-66 $\left(\mathrm{T}_{\mathrm{g}}\right.$ aPP=258K $\left.)\right]$. As we saw in the previously presented data, the PIB backbone mobility is enhanced by blending it with the higher $T_{g}$ component, even though it is the lower $T_{g}$ polymer. Also, PIB has a much higher density of $0.91 \mathrm{~g} / \mathrm{cm}^{3}$ when compared with polypropylene of around $0.85 \mathrm{~g} / \mathrm{cm}^{3}$, which is similar to most other polyolefins. ${ }^{11}$ Our future investigations on blends with polypropylene will determine if we can draw comparisons between blends of aPP/PEBs and the PIB/PEBs systems, even though the PIB properties were unusual.

In the PIB/PEB system, our next step included two-dimensional exchange NMR studies combined with ${ }^{2} \mathrm{H}$ NMR. ${ }^{13}$ However, for PP/PE blends the resolution in the $2 \mathrm{D}$ experiments is insufficient as pointed out above. Another disadvantage in the $2 \mathrm{D}$ exchange experiment is the narrow temperature range within which the signal is useful due to the fact that at high temperatures, rapid conformer exchange eliminates the ability to follow main-chain dynamics, while at temperatures below the glass transition, the exchange is slow relative to spinlattice relaxation. The new strategy with the polypropylene systems includes expanding to another experiment, the CODEX, to provide the same slow segmental dynamics data at the molecular level.

\section{CODEX}

As discussed in Chapter 2, CODEX is based on recoupling the chemical shift anisotropy to detect dynamic processes occurring during the mixing time. ${ }^{63}$, 
If molecular motions took place during the allotted time, the signal is incompletely refocused causing a decrease in the signal intensity of the CODEX spectrum as compared with the reference spectrum. The entire experiment consists of running a reference and CODEX spectrum with interchanging the two mixing values $\left(t_{m}\right.$ and $t_{z}$ as seen in Figure 2-11) and later subtracting the two. An example of amorphous aPP sample at $260 \mathrm{~K}$ is demonstrated in Figure 7-6, with
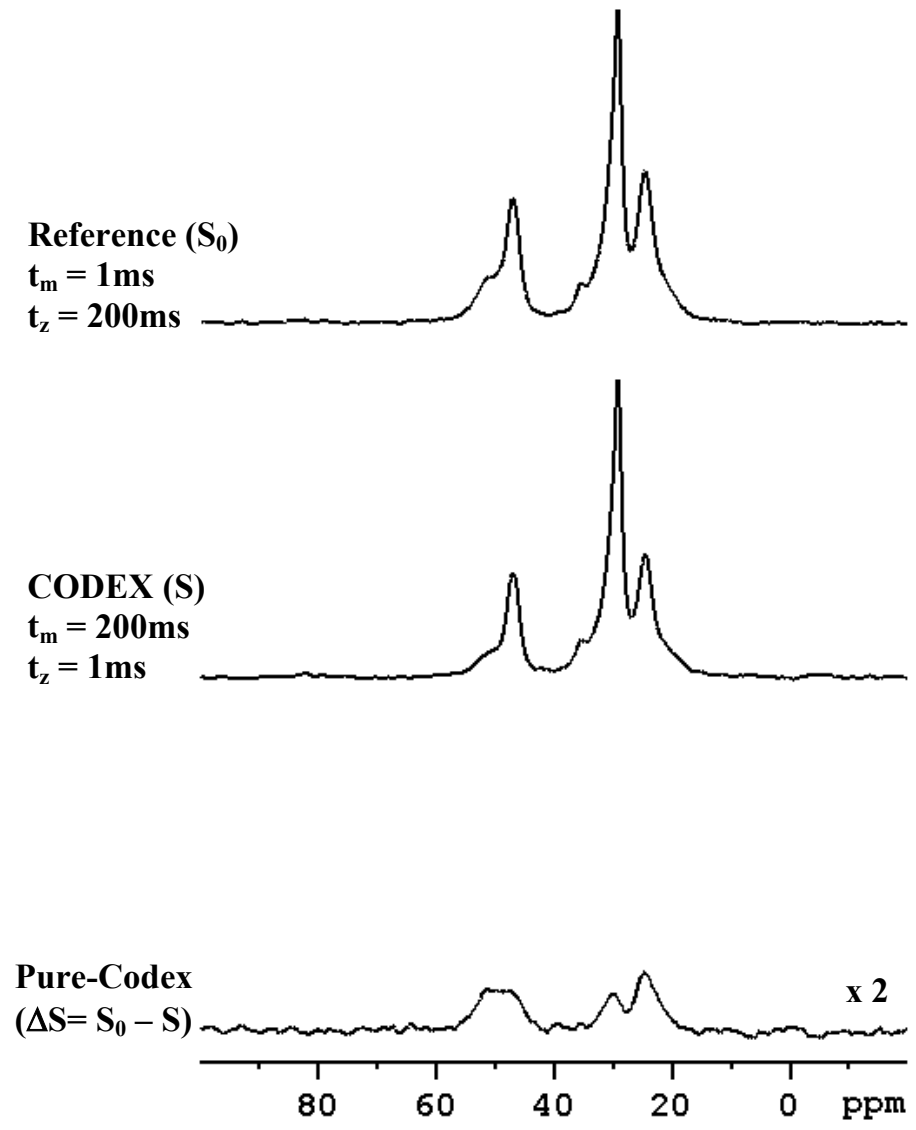

Figure 7-6 Three spectra of aPP at 260K are displayed indicating how the pure-CODEX spectrum is obtained. The pure-CODEX spectra signal was magnified by two.

the reference, CODEX, and pure-CODEX spectrum indicated. The difference or 'pure-CODEX' spectrum has relaxation effects removed, making the present 
signal representative of the carbons undergoing molecular motion. Major advantages of the recently developed CODEX experiment include high sensitivity to dynamic process of carbons with small chemical shift anisotropies, maximum spectral resolution, no labeling required, and the capability to add specialized filters to discriminate signal via $\mathrm{T}_{1}$ or $\mathrm{T}_{2}{ }^{63,64}$ Again, as pointed out in Chapter 2, the primary focus with CODEX was pulse sequence calibration on static and mobile control samples and satisfying experimental demands such as block averaging and rotor synchronization. Methylmalonic acid served as the static molecule, giving a null pure-CODEX spectrum whereas bisphenol-A $\left(4,4^{\prime}\right.$ isopropylidenediphenol) displayed reorientations with tens of hertz correlation time. ${ }^{64}$ Consequently, dynamics data can be observed for multiple peaks from both polymers in the blend at high resolution, thus enhancing the total (exchange) information content relative to the $2 \mathrm{D}$ approach. More importantly, data at temperatures above and below the blend critical solution temperature may be acquired. One major advantage of the $2 \mathrm{D}$ exchange over the CODEX is that the types of molecular rearrangements are clearer from the former, as we saw with PIB demonstrating the conformational interchange between trans-trans, transgauche, and gauche-gauche conformations.

Dynamics processes taking place in pure aPP and PEB-66 versus their blend, aPP/PEB-66, were probed using a combination of the CODEX and variable temperature CPMAS. Variable temperature pure-CODEX spectra of aPP (a), aPP/PEB-66 (b), and PEB-66 (c) at mixing times of 100ms or 200ms are shown in Figure 7-7. The room temperature conditions prevent good signal-to-noise 
spectra (the spectra have distorted phasing) to be detected because of the fast motion that averages the small carbon CSAs. A significant signal improvement is detected as the temperature is lowered due to slower dynamics, as seen by the spectrum of pure aPP at $260 \mathrm{~K}$ (a). The glass transition of aPP is around $258 \mathrm{~K}$, while PEB-66 has a lower $T_{g}(221 \mathrm{~K})$. From the plots in Figure 7-7, signal is observed right around the $T_{g}$ of the samples, and once the temperature is

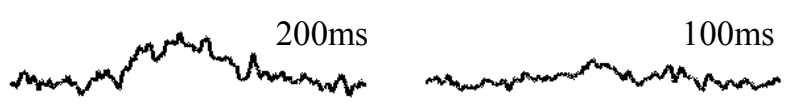

$230 K$
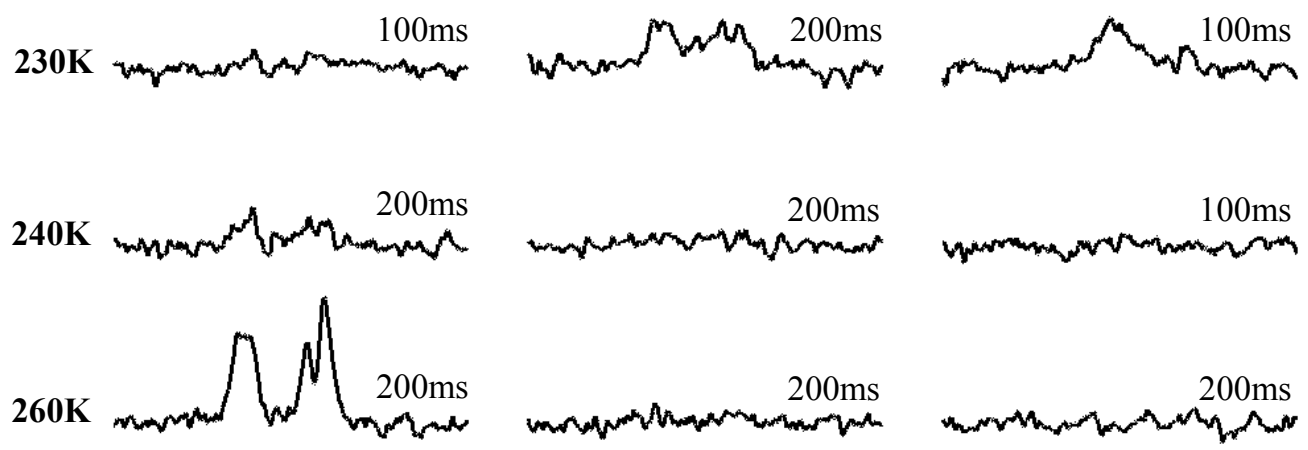

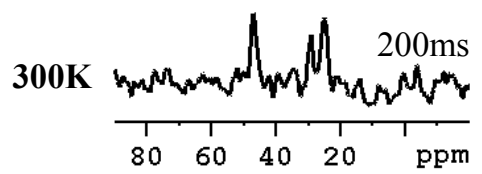

(a)

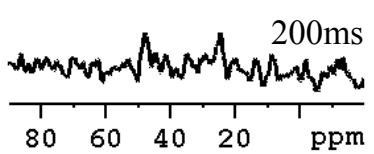

(b)

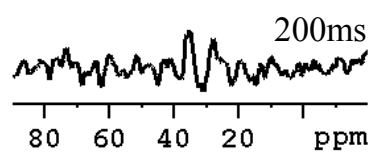

(c)

Figure 7-7 Pure-CODEX spectra for (a) pure aPP, (b) aPP/PEB-66 and (c) PEB-66. All represent either $100 \mathrm{~ms}$ or $200 \mathrm{~ms}$ exchange times acquired with $12 \mathrm{~K}$ scans and line-broadening of $75 \mathrm{~Hz}$.

decreased below the $T_{g}$, the signal disappears. The combination of the CSA and slow molecular reorientations at 100 -ms or 200 -ms mixing times result in signal 
for aPP at $260 \mathrm{~K}$ and PEB-66 at both $220 \mathrm{~K}$ and $230 \mathrm{~K}$. In order to prove a change in the chain-level dynamics of the two components from blending, the ideal result would display signal in both components while none in the blend, or vice versa. By being able to achieve such conditions, we would show that the dynamics of polymer chains are altered significantly upon mixing the two polymers. The temperatures examined in Figure 7-7 showed no such conditions could be detected, because either both aPP and the blend showed signal, or PEB-66 and the blend at the same time. Subsequently, we went back to a former miscible blend to determine whether perturbation on the molecular level could be detected via this method.

Chain-level dynamics in PIB and the intimately mixed blend PIB/hhPP, have been well documented with xenon NMR as well as ${ }^{1} \mathrm{H}$ spin-diffusion experiments, demonstrating miscibility on a length scale of $2.8 \mathrm{~nm}^{33,}{ }^{138}$ The advantage with this system is that there is a distinct peak from PIB (methylene) which is sensitive to relative rates of conformational exchange along the backbone as observed in the variable temperature CP/MAS plot in Figure 7-8. Yet unfortunately, the stackplot is not as informative as it was for the miscible PIB/PEB-66 blend in Figure 4-2, ${ }^{13}$ displaying almost identical lineshape changes in the pure PIB in Figure 7-8 (a) as in the blend in part (b). As seen in the plots, in both cases the Rothwell-Waugh effect takes place roughly between $253 \mathrm{~K}$ 263K. Relatively no differences can be distinguished to indicate mixing-induced alterations in dynamics on the ten of $\mathrm{kHz}$ frequency scale. Figure 7-8 (c) represents the pure hhPP sample, and upon comparing the methyl signal over the 
large temperature range with the methyl region for the blend in (b), a large difference can be detected. The populations selected in the cross-polarization step

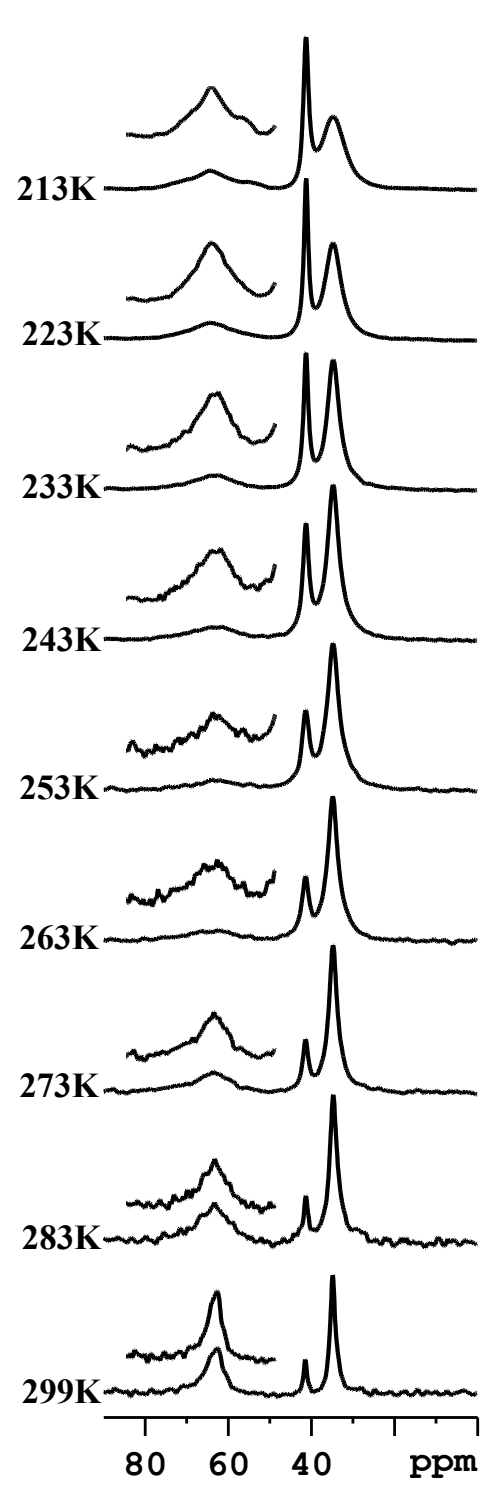

(a)

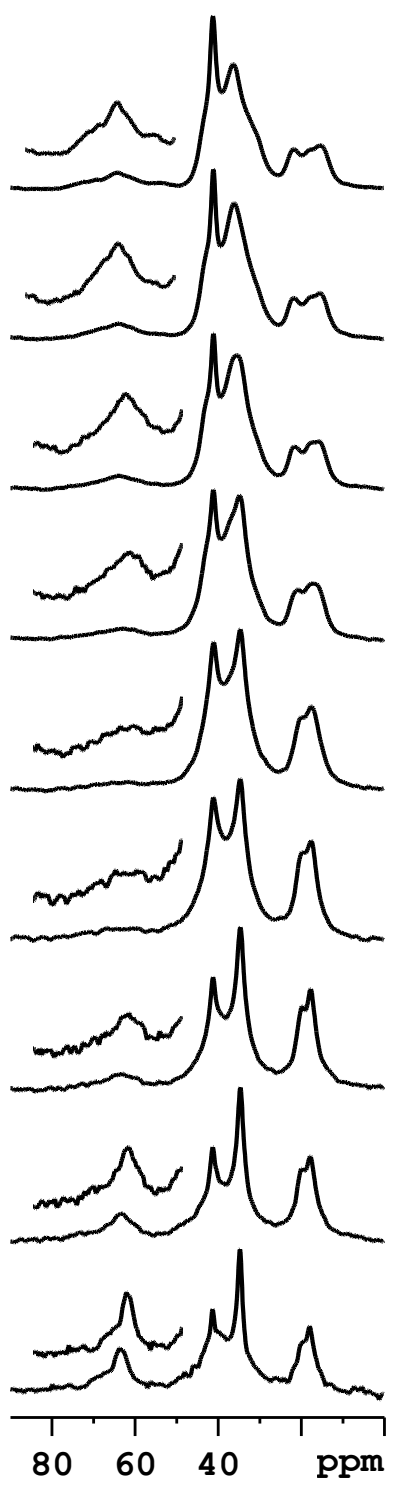

(b)

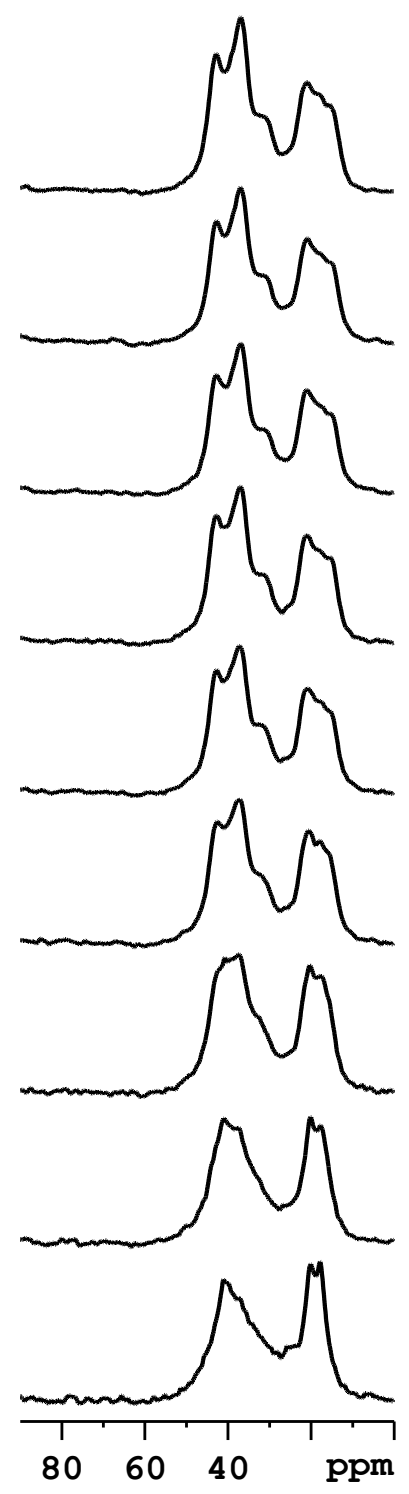

(c)

Figure 7-8 Variable temperature cross-polarization stackplots comparing the (a) pure PIB versus (b) PIBhhPP $\left(5 \%{ }^{13} \mathrm{C}\right.$ labeled $)$ and (c) hhPP. 
differ for the blend and the pure hhPP component, but no explanation for the selection can be offered at this time. Therefore, the inconclusive results offered with VT CPMAS suggest that another experimental approach such as CODEX is potentially useful.

A CODEX plot, comparing the two pure materials and the blend of $\mathrm{PIB} / \mathrm{hhPP}$, is shown in Figure 7-9. The glass transitions are both low for PIB $\left(\mathrm{T}_{\mathrm{g}}\right.$
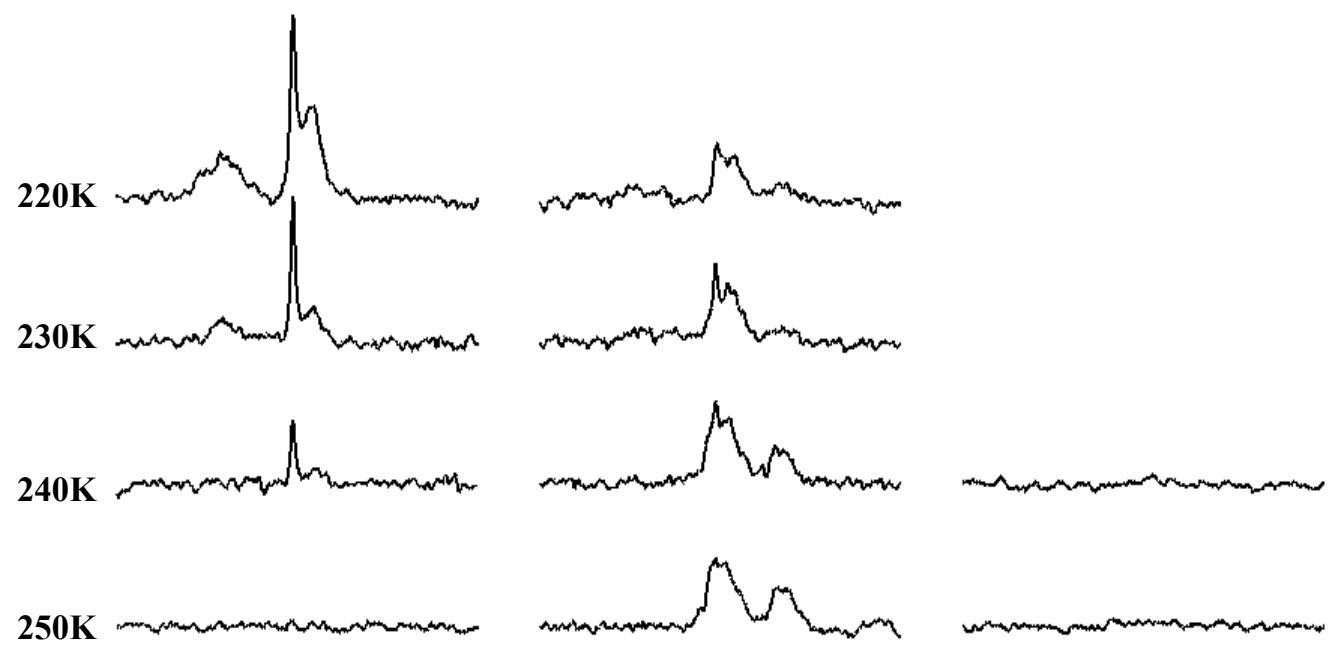

$250 \mathrm{~K}$
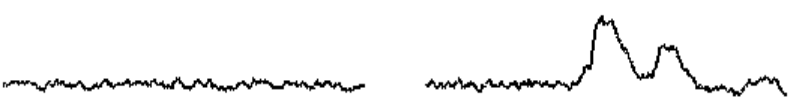

$260 K$
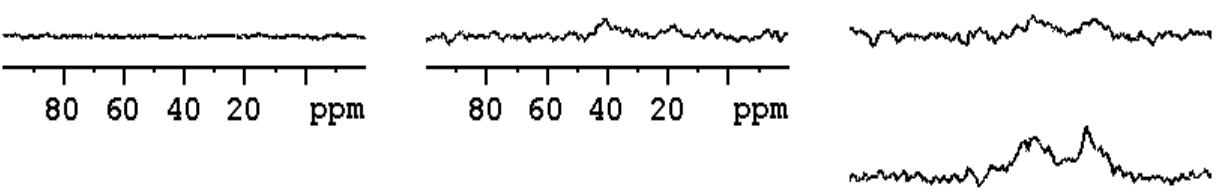

270K

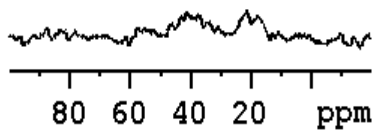

(a)

(b)

(c)

Figure 7-9 Pure-CODEX spectra for (a) PIB, (b) PIB/hhPP, and (c) hhPP at various temperatures as indicated. All spectra were run at a mixing time of $100 \mathrm{~ms}$ and $12 \mathrm{~K}$. All the spectra at $260 \mathrm{~K}$ were magnified by two, while the rest were plotted on the same scale. 
$=203 \mathrm{~K})$ and $\mathrm{hhPP}\left(\mathrm{T}_{\mathrm{g}}=253 \mathrm{~K}\right)$, thus the signal to noise shows a large increase as the temperature is lowered beyond room temperature (signal was either absent or distorted at room temperature due to fast motion). The signal differs from the aPP/PEB-66 system in that the pure-CODEX spectra signal persists over a larger temperature range, at least for the pure PIB (signal observed at three temperatures) and the blend (signal observed at all five temperatures examined). The data portraying the differential dynamics that occurs with blending can be seen at $250 \mathrm{~K}$, where both pure components demonstrate no pure-CODEX signal, whereas significant signal can be seen for the blend. More specifically, we observe decaying signal for pure PIB as the temperature is increased above its $T_{g}$ due to the increasing molecular motions taking place. Whereas, hhPP has the higher $\mathrm{T}_{\mathrm{g}}$, and signal is suppressed for the majority of the low temperatures, and begins to show around $260 \mathrm{~K}$, close to its glass transition. But in the blend, the chain-level changes that take place with the blending process, are portrayed with large signal due to the slow-molecular reorientations occurring. If no changes with blending took place in the individual polymer segments, we would have observed the same signal as we did for the pure components.

The utility of CODEX demonstrated above will be further pursued in our other systems. In addition, an expansion of the experiment will be to determine the actual chemical shift anisotropies and quantifying the data so as to establish reorientation angles. Together with these new techniques, our old methodology will be applied to the systems as already illustrated in this chapter. 


\section{CONCLUSIONS AND FUTURE WORK}




\section{Overall Conclusions}

The purpose for investigating the molecular origins of miscibility in polyolefins, is driven by their potential technological impact, alongside a large economic importance for these polymer systems. New polyolefin blend compositions are becoming available due to novel polymer architectures from metallocene catalysis, but development of a thorough understanding of factors surrounding compatibility (such as length scales of mixing, local packing and chain dynamics as the main objectives) is required for predicting new material compositions. The groundwork for determining miscibility windows in polyolefin blends is outlined in this work, which demonstrated that solid-state NMR is a valuable tool in polymer blend science. Topics concerning dynamics in blends as well as domain sizes (i.e. length scales of mixing) can be tackled through various experimental combinations, because of this method's extreme sensitivity to local conformers and slight differences in molecular level structure. In addition, isotopic labeling is not a prerequisite requirement in SSNMR, thereby rendering our results directly applicable to industrial applications. The research community is seeking answers to the major questions governing factors influencing miscibility, e.g. enhanced chain packing (enthalpy), increased local dynamics/conformational populations (entropy), or a complex interplay between

the two. At this time, the lack of molecular- or chain-level data is a limiting factor. Our immediate goals are to design SSNMR experimental combinations, along with accurate data interpretation techniques, that will offer precise descriptions of polyolefin blend phase behavior. Consequently, the acquired 
knowledge and understanding will aid in 'engineering' desired phase behavior, thus allowing improved physical properties via polyolefin blends to be realized.

More specifically, the detailed local data acquired in this work revealed information regarding the local packing, dynamics, and length scales of mixing in amorphous systems such as $\mathrm{PIB} / \mathrm{PEBs}, \mathrm{PIB} / \mathrm{hhPP}$ and aPP/PEBs. The focus remained on PIB throughout this work due to its anomalous behavior (large negative interaction parameters) compared with other polyolefins. Our group was the first to provide chain level, experimental data as evidence for configurational entropy being the driving force for miscibility in certain polyolefin blends. We were able to conclude that miscibility in the PIB/PEB-66 blend is not due to an increase in chain packing for the blend, or in other words, not due to enhanced enthalpy arising from increased cumulative van der Waals forces. Flory-Huggins theory discounts entropy as a possible contributing factor to mixing, and it simply does not treat the polymer at the chain level where configurational entropy contributions are important. Hence, our efforts lie in collecting chain-level data and to consider configurational entropy as a vital force influencing polymer mixing, something which has received little attention. Future experimental goals are to expand to blends of wider economic importance, such as PP/PE, and see if our theory will work for similar systems. 


\section{Further Work}

The chief aim of this project is to obtain molecular level data in order to understand how 'non-associating' polymers associate with one another. In this work, SSNMR methodology was devised and applied primarily to the PIB/PEBs and PIB/hhPP systems. However, as we have already seen, each individual binary blend will pose its own challenges, thus requiring new experiments and innovative approaches. For example, the aPP/PEB systems have smaller signal dispersions, requiring a substitution of the two-dimensional exchange experiment with the CODEX to obtain high quality dynamic information at the best possible resolution of multiple partially overlapped peaks. Also, the novel aPP/PEB systems introduce local structural variations via tacticity in atactic polypropylene. Hence when added together with the copolymer sequence combinations, they combine to produce diverse local dynamics. (Previously, only the copolymer combinations were introduced with no tacticity changes from PIB). As a result, with each new system within the area of polyolefin blends, experimental approaches must be re-evaluated.

Ongoing experiments will address determining xenon diffusion coefficients in helping to determine exact domain sizes. As seen above for our PIB/PEB-66 system, we were able to obtain accurate xenon diffusion coefficients through our collaboration with DuPont. Due to the lack of strong gradients in our labs, we will purse further xenon diffusion coefficients with our collaborators on the upcoming new systems to obtain the most accurate possible domain-size estimates. 
Novel techniques that we would like to become routine in our polyolefin blend miscibility pursuits are two-dimensional simulations. Simulating patterns that are observed experimentally would enable extractions of molecular processes taking place. In addition, they could be supportive in identifying preferential pathways of exchange for individual chain components in the miscible blends. For example, we were fortunate in our $2 \mathrm{D}$ exchange experiments in investigating the PIB glass transition, when we were able to identify the first conformer exchange taking place. Two-dimensional simulations could make the process much simpler.

A new expansion of our work will include blends with i-PP, a semicrystalline material which when blended with amorphous polyolefin can offer attractive commercial properties such as high impact strength. Phase behavior is expected to be more difficult to study, because upon cooling the blend from a melt to a solid, miscibility can be expected for the amorphous regions but is unlikely for the crystalline region. Yet, there are recent reports, which show miscibility in the melt of i-PP with copolymers like ethylene-butene and ethylenehexene demonstrating that the right amount of comonomer could produce singlephase amorphous regions in the solid state. A possible explanation could be an alteration in the i-PP crystallite sizes that occurs with cooling for a miscible blend, which results in changes to the crystallization kinetics. SSNMR can be significant in studying these 'partially' miscible blends because various experiments can be used to discriminate between the amorphous and crystalline polymer chains and determine how close they are to one another. Further studies and comparisons can 
be made to determine the differences between blends using aPP versus iPP, and outline the differences in their miscibility windows.

As discussed in previous chapters, using $2 \mathrm{D}$ exchange NMR, we began investigating the glass transition phenomena, pointing out for example, the first molecular transition taking place. We would like to further explore questions regarding both the length scales and times scales of the glass transition. Glass formation has been described in the literature as resulting from configurational entropy losses, and we would like to see if our polyolefin blend systems can provide non-invasive, chain level proof. The architecturally diverse polyolefins are ideal systems to study and explore these concepts via the controlled, systematic introduction of local chain dynamics (by increasing the comonomer amounts), and thus, possible methodical observations in dynamic fluctuations of the time scale and length scales. By completing the discussed studies, we hope to be able to contribute to a refined theory of polymer miscibility. 


\section{REFERENCES}


1. Paul, D. R.; Bucknall, C. B., Polymer Blends: Formulation and

Performance. ed.; Wiley Publishers: New York, 2000; 'Vol.' 1, p 219.

2. Online Reference. http://www.pslc.ws/macrog.

3. Kaminsky, W. J. Polym. Sci., Part A: Polym. Chem 2004, 42, 3911.

4. $\quad$ Sinclair, K. B. Hydrocarbon Processing 2004, 83, 39.

5. Hlatkey, G. G. Chem. Rev. 2000, 100, 1347.

6. Chem. \& Eng. News 2000, 78, 35 .

7. Chem. \& Eng. News 2000, 78, 39.

8. Crist, B.; Hill, M. J. J. Polym. Sci., Part B: Polym. Phys 1997, 35, 2329.

9. Schmidt-Rohr, K.; Spiess, H. W. Multidimensional Solid-State NMR and Polymers; Academic Press: New York, 1994.

10. Bates, F. S.; Kumar, A.; Schulz, M. F. J. Polym. Sci., Part B: Polym. Phys 1995, 33, 1423.

11. Krishnamoorti, R.; Graessley, W. W.; Fetters, L. J.; Garner, R. T.; Lohse, D. J. Macromolecules 1995, 28, 1252.

12. Reichart, G. C.; Register, R. A.; Graessley, W. W.; Krishnamoorti, R.; Lohse, D. J. Macromolecules 1997, 30, 3036.

13. Wolak, J. E.; Jia, X.; Jurga, S. J.; Gracz, H.; Stejskal, E. O.; White, J. L. Macromolecules 2003, 36, 4844.

14. Graessley, W. W.; Krishnamoorti, R.; Reichart, G. C.; Balsara, N. P.; Fetter, L. J.; Lohse, D. J. Macromolecules 1995, 28, 1260.

15. Krishnamoorti, R.; Graessley, W. W.; Dee, G. T.; Walsh, D. J.; Fetters, L. J.; Lohse, D. J. Macromolecules 1996, 29, 367.

16. Reichart, G. C.; Graessley, W. W.; Register, R. A.; Lohse, D. J. Macromolecules 1998, 31, 7886.

17. Bates, F. S.; Fredrickson, G. H. Macromolecules 1994, 27, 1065.

18. Wolak, J. E.; Jia, X.; White, J. L. J. Am. Chem. Soc. 2003, 125, 13660.

19. Richer, R.; Angell, C. A. J. Chem. Phys. 1998, 108, 9016.

20. Sivaram, S.; Chandrasekhar, S. B. Current Science 2000, 78, 1325.

21. Kristen, M. Topics in Catalysis 1999, 7, 89.

22. Sinclair, K. B.; Wilson, R. B. Chemistry and Industry 1994, 21, 857.

23. Carraher, C. E. Polymer Chemistry; Marcel Dekker, Inc.: New York, 1996.

24. Chien, J. C. W. Topics in Catalysis 1999, 7, 23.

25. Hiemenz, P. C. Polymer Chemistry; Marcel Dekker, Inc.: New York, 1984.

26. Tester, J. W.; M.Modell Thermodynamics and Its Applications; PrenticeHall, Inc.: Upper Saddle River, NJ, 1997.

27. Krishnamoorti, R.; Graessley, W. W.; Balsara, N. P.; Lohse, D. J. Macromolecules 1994, 27, 3073.

28. Reichart, G. C.; Register, R. A.; Graessley, W. W.; Krishnamoorti, R.; Lohse, D. J. Macromolecules 1995, 28, 8862.

29. Weimann, P. A.; Jones, T. D.; Hillmyer, M. A.; Bates, F. S. Macromolecules 1997, 30, 3650.

30. Almdal, K.; Hillmyer, M. A.; Bates, F. S. Macromolecules 2002, 35, 7685. 
31. Balsara, N. P.; Fetters, L. J.; Hadjichristdis, N.; Lohse, D. J.; Graessley, W. W.; Krishnamoorti, R. Macromolecules 1992, 25, 6137.

32. Graessley, W. W.; Krishnamoorti, R.; Balsara, N. P.; Fetter, L. J.; Lohse, D. J.; Schulz, D. N.; Sissano, J. A. Macromolecules 1993, 26, 1137.

33. White, J. L.; Lohse, D. J. Macromolecules 1999, 32, 958.

34. Wang, X.; White, J. L. Macromolecules 2002, 35, 3795.

35. White, J. L.; Brant, P. Macromolecules 1998, 31, 5424.

36. White, J. L.; Schaffer, T. D.; Ruff, C. J. Macromolecules 1995, $28,3290$.

37. Laws, D. D.; Bitter, H. M. L.; Jerschow, A. Angew. Chem. Int. Ed. 2002, 41, 3096.

38. Komorowski, R. A. High Resolution NMR Spectroscopy of Synthetic Polymers in Bulk; VCH Publishers: Deerfield Beach, 1986.

39. Kobori, Y.; Akiba, I.; Akiyama, S.; Ishikawa, K. European Polymer Journal 2000, 36, 503.

40. Tonelli, A. E.; Schilling, F. C. Acc. Chem. Res. 1981, 14, 233.

41. Tonelli, A. E. J. Mol. Struct. 1995, 355, 105.

42. Zemke, K.; Schmidt-Rohr, K.; Spiess, H. W. Acta Polym. 1994, 45, 148.

43. Rudin, A. Polymer Science and Engineering; Academic Press: San Diego, CA., 1999.

44. Bielecki, A.; Burum, D. P. J. Magn.Reson. 1995, 116, 215.

45. Yannoni, C. S. Acc. Chem. Res. 1982, 15, 201.

46. Lyerla, J. R.; Yannoni, C. S. Acc. Chem. Res. 1982, 15, 208.

47. Stejskal, E. O.; Schaefer, J.; Sefcik, R. A.; McKay, R. A. Macromolecules 1981, 14, 275.

48. White, J. L.; Dias, A. J.; Ashbaugh, J. R. Macromolecules 1998, 31, 1880.

49. $\quad$ Schaefer, J.; Stejskal, E. O.; Buchdahl, R. Macromolecules 1975, 8, 291.

50. Spiess, H. W. Chem. Rev. 1991, 91, 1321-1338.

51. Andrew, E. R. Prog. Nucl. Magn. Reson. Spectrosc. 1971, 8, 1.

52. Lowe, I. J. Phys. Rev. Lett. 1959, 2, 285.

53. Schneider, B.; Doskocilova, D.; Babka, J.; Ruzicka, Z. J. Magn.Reson. 1980, 37, 41.

54. Hartmann, S. R.; Hahn, E. L. Phys. Rev. 1962, 128, 2042.

55. Pines, A. P.; Gibby, M. G.; Waugh, J. S. J. Chem. Phys. 1973, 59, 569.

56. Rothwell, W. P.; Waugh, J. S. J. Chem. Phys. 1981, 74, 2721.

57. Suwelack, D.; Rothwell, W. P.; Waugh, J. S. J. Chem. Phys. 1980, 73, 2559.

58. Maricq, M. M.; Waugh, J. S. J. Chem. Phys. 1979, 70, 3300.

59. Blumich, B.; Spiess, H. W. Angew. Chem. Int. Ed. Engl. 1988, 27, 1655.

60. Kentgens, A. P. M.; DeJong, A. F.; DeBoer, E.; Veeman, W. S. Macromolecules 1985, 18, 1045.

61. Zemke, K.; Chmelka, B. F.; Schmidt-Rohr, K.; Spiess, H. W. Macromolecules 1991, 24, 6874.

62. Kentgens, A. P. M.; Boer, E. d.; Veeman, W. S. J. Chem. Phys. 1987, 87, 6859 . 
63. DeAzevedo, E. R.; Hu, W. G.; Bonagamba, T. J.; Schmidt-Rohr, K. J. Chem. Phys. 2000, 112, 8988.

64. DeAzevedo, E. R.; Hu, W. G.; Bonagamba, T. J.; Schmidt-Rohr, K. J. Am. Chem. Soc. 1999, 121, 8411.

65. Miyoshi, T.; Pascui, O.; Reichert, D. Macromolecules 2004, 37, 6460.

66. Bonagamba, T. J.; Becker-Guedes, F.; DeAzevedo, E. R.; Schmidt-Rohr, K. J. Polym. Sci., Part B: Polym. Phys 2001, 39, 2444.

67. DeAzevedo, E. R.; Franco, R. W. A.; Marletta, A.; Faria, R. M.; Bonagamba, T. J. J. Chem. Phys. 2003, 119, 2923.

68. Miyoshi, T.; Pascui, O.; Reichert, D. Macromolecules 2002, 35, 7178.

69. Reichert, D.; Bonagamba, T. J.; Schmidt-Rohr, K. J. Magn.Reson. 2001, 151, 129.

70. Wolak, J. E.; Knutson, J.; Martin, J. D.; Boyle, P.; Sargent, A. L.; White, J. L. J. Phys. Chem. B 2003, 107, 13293.

71. Duer, M. J. Solid-State NMR Spectroscopy Principles and Applications; Blackwell Science Ltd.: Malden, 2002.

72. Bovey, F. A.; Mirau, P. E. NMR of Polymers; Academic Press, Inc.: San Diego, 1996.

73. Simpson, J. H.; Rice, D. M.; Karasz, F. E. J. Polym. Sci., Part B: Polym. Phys 1992, 30, 11.

74. Schantz, S.; Veeman, W. S. J. Polym. Sci., Part B: Polym. Phys. 1997, 35, 2681.

75. Stengle, T. R.; Williamson, K. L. Macromolecules 1987, 20, 1428-1430.

76. Walton, J. H.; Miller, J. B.; Roland, C. M. J. Polym. Sci., Part B: Polym. Phys. 1992, 30, 527-532.

77. Mirabella, F. M.; McFaddin, D. C. Polymer 1996, 37, 931-938.

78. Junker, F.; Veeman, W. S. Macromolecules 1998, 31, 7010.

79. Walton, J. H.; Miller, J. B.; Roland, C. M.; Nagode, J. B. Macromolecules 1993, 26, 4052.

80. Stejskal, E. O.; Tanner, J. E. J. Chem. Phys. 1965, 42, 288.

81. Springuel-Huet, M. A.; Bonardet, J. L.; Gedeon, A.; J.Fraissard. Magn. Reson. Chem. 1999, 37, 1-13.

82. Atkins, P. Physical Chemistry; Freeman: New York, 1999.

83. Chem. \& Eng. News 2003, 81, 40.

84. Bel'skii, V. K.; Chernikova, N. Y.; Rotaru, V. K.; Kruchinin, M. M. Sov. Phys. Crystallogr. 1983, $28,405$.

85. Okada, K. J. Mol. Struct. 1996, 380, 223.

86. X-ray data (lists of atomic coordinates, t. p., structure factors, etc.) acquired at $-60 \mathrm{C}$ is provided as supporting information (cif file).

87. Schaefer, J.; Stejskal, E. O.; McKay, R. A.; Dixon, W. T. Macromolecules 1984, 17, 1479.

88. Lee, P. L.; Schaefer, J. Macromolecules 1992, 25, 5559.

89. Schmidt, A.; Schaefer, J. Macromolecules 1993, 26, 1729.

90. Kaji, H.; Tai, T.; Horii, F. Macromolecules 2001, 34, 6318. 
91. Roy, A. K.; Jones, A. A.; Inglefield, P. T. Macromolecules 1986, 19, 1356.

92. Spiess, H. W. Advances in Polymer Science; Springer-Verlag: Berlin, 1984.

93. Shi, J. F.; Jones, A. A.; Inglefield, P. T.; Meadows, M. D. Macromolecules 1996, 29, 605.

94. Henrichs, P. M.; Russ, H. R. Macromolecules 1988, 21, 860.

95. Henrichs, P. M.; Russ, H. R.; Scaringe, R. P. Macromolecules 1989, 22, 2731.

96. Szeverenyi, N.; Sullivan, M. J.; Maciel, G. E. J. Magn.Reson. 1982, 47, 462.

97. Hagemeyer, A.; Schmidt-Rohr, K.; Spiess, H. W. Adv. Magn. Reson. 1989, $13,85$.

98. Suter, D.; Ernst, R. R. Phys. Rev. B 1985, 32, 5608.

99. Rossum, B. J. V.; Groot, C. P. D.; Ladishansky, V.; Vega, S.; Groot, H. J. M. D. J. Am. Chem. Soc. 2000, 122, 3465.

100. Frisch, M. J. T., G. W.;Schlegel, H. B.;Scuseria, G. E.;Robb, M. A.;Cheeseman, J. R.;Zakrzewski, V. G.; Montgomery, Jr., J. A.; Stratmann, R. E.; Burant, J. C.; Dapprich, S.; Millam, J. M.; Daniels, A. D.; Kudin, K. N.; Strain, M. C.; Farkas, O.; Tomasi, J.; Barone, V.; Cossi, M.; Cammi, R.; Mennucci, B.; Pomelli, C.; Adamo, C.; Clifford, S.; Ochterski, J.; Petersson, G. A.; Ayala, P. Y.; Cui, Q.; Morokuma, K.; Malick, D. K.; Rabuck, A. D.; Raghavachari, K.; Foresman, J. B.; Chioslowski, J.; Ortiz, J. V.; Baboul, A. G.; Stefanov, B. B.; Liu, G.; Liashenko, A.; Piskorz, P.; Komaromi, I.; Gomperts, R.; Martin, R. L.; Fox, D. J.; Keith, T.; Al-Laham, M. A.; Peng, C. Y.; Nanayakkara, A.; Challacombe, M.; Gill, P. M. W.; Johnson, B.; Chen, W.; Wong, M. W.; Andres, J. L.; Gonzalez, C.; Head-Gordon, M.; Replogle, E. S.; Pople, J. A. Gaussian 98, Revision A.9; Gaussian, Inc.: Pittsburgh, PA, 1998.

101. Hariharan, P. C.; Pople, J. A. Theor. Chim. Acta 1973, 28, 213.

102. Peng, C.; Schlegel, H. B. Israel J. Chem. 1993, 33, 449.

103. Folmer, J.; Franzen, S., private communication.

104. Harper, J. K.; Facelli, J. C.; Barich, D. H.; McGeorge, G.; Mulgrew, A. E.; Grant, D. M. J. Am. Chem. Soc. 2002, 124, 10589.

105. Helluy, X.; Sebald, A. J. Phys. Chem. B 2003, 3290.

106. Clauss, J.; Schmidt-Rohr, K.; Spiess, H. W. Acta Polym. 1993, 44, 1.

107. VanderHart, D. L. Macromol. Chem., Macromol. Symp. 1990, 38, 125.

108. VanderHart, D. L.; McFadden, G. M. Solid State NMR 1996, 7, 45.

109. Mellinger, F.; Wilhelm, M.; Spiess, H. W. Macromolecules 1999, 32, 4686.

110. Egger, N.; Schmidt-Rohr, K.; Blumich, B.; Domke, W. D.; Stapp, B. J. Appl. Polym. Sci. 1992, 44, 289.

111. Goldman, M.; Shen, L. Phys. Rev. 1966, 144, 321.

112. Spiess, H. W. Colloid Polym. Sci. 1983, 261, 193. 
113. Walton, J. H.; Miller, J. B.; Roland, C. M. J. Polym. Sci., Part B: Polym. Phys 1995, 33, 1423.

114. Boyd, R. H.; Pant, P. V. K. Macromolecules 1992, 25, 494.

115. Sanchez, I. C.; Stone, M. T., In Polymer Blends, ed.; 'Ed.'^'Eds.' Wiley and Sons: New York, 2000; 'Vol.' p^pp 15.

116. Corezzi, S.; Floretto, D.; Rolla, P. Nature 2002, 420, 653.

117. Tracht, U.; Wilhelm, M.; Heuer, A.; Feng, H.; Schmidt-Rohr, K.; Spiess, H. W. Phys. Rev. Lett. 1998, 81, 2727.

118. Ediger, M. D. J. Phys. Chem. B 1999, 103, 4177.

119. Hempel, E.; Hempel, G.; Hensel, A.; Schick, C.; Donth, E. J. Phys. Chem. $B$ 2000, 104, 2460.

120. Schmidt-Rohr, K. Multidimensional Spectroscopy of Polymers; ACS Symp. Ser. 598, Chapter 12, p191: 1995.

121. Beckham, H. W.; Schmidt-Rohr, K.; Spiess, H. W. Multidimensional Spectroscopy of Polymers; ACS Symp. Ser. 598 Chapter 14: 1995.

122. Schaefer, D.; Spiess, H. W.; Suter, U. W.; Fleming, W. W. Macromolecules 1990, 23, 3431.

123. Heuer, A.; Wilhelm, M.; Zimmermann, H.; Spiess, H. W. Phys. Rev. Lett. 1995, 75, 2851.

124. Adam, G.; Gibbs, J. H. J. Chem. Phys. 1965, 43, 139.

125. Kant, R.; Kumar, A.; Colby, R. H. Macromolecules 2003, 36, 10087.

126. Lodge, T. P.; McLeish, T. C. Macromolecules 2000, 33, 5278.

127. Huang, D.; Simon, S. L.; McKenna, G. B. J. Chem. Phys. 2003, 119, 3590.

128. Jones, R. A. L. Nature (London) 2002, 2, 645.

129. Donth, E. J. Polym. Sci., Part B: Polym. Phys 1996, 34, 2881.

130. Bennenmann, C.; Donati, C.; Baschnagel, J.; Glotzer, S. C. Nature (London) 1999, 399, 246.

131. Ediger, M. D.; Skinner, J. L. Science 2001, 292, 233.

132. Deschenes, L. A.; Bout, D. A. V. Science 2001, 292, 255.

133. Tanaka, H. J. Chem. Phys. 1996, 105, 9375.

134. Macho, V.; Brombacher, L.; Spiess, H. W. Appl. Magn. Reson. 2001, 20, 405.

135. Hirschinger, J.; English, A. D. J. Magn.Reson. 1989, 85, 542.

136. Schadt, R. J.; Cain, E. J.; English, A. D. J. Phys. Chem. 1993, 97, 8387.

137. Bondi, A. J. Phys. Chem. 1964, 68, 441.

138. White, J. L.; Wang, X. Macromolecules 2002, 35, 3795.

139. Wu, D.; Chen, A.; Johnson, C. S. J. Magn.Reson. 1995, 115, 260.

140. Johnson, C. S. Prog. Nucl. Magn. Reson. Spectrosc. 1999, 34, 203.

141. Wachowicz, M.; Wolak, J. E.; Gracz, H.; Stejskal, E. O.; Jurga, S. J.; McCord, E. F.; White, J. L. Macromolecules 2004, 37, 4573. 


\section{APPENDIX}

A-1 Pulse Sequence: 2D ${ }^{13} \mathrm{C}-{ }^{13} \mathrm{C}$ Exchange NMR

A-2 Pulse Sequence: Centerband-Only Detection of Exchange (CODEX)

A-3 Supporting Information (.cif file): Bisphenol-A coordinates from $-60^{\circ}$ single- crystal X-ray experiments 


\section{A-1 Pulse Sequence: 2D ${ }^{13} \mathrm{C}-{ }^{13} \mathrm{C}$ Exchange NMR}

;jwcpnoesy.98

;2D exchange NMR in rotating solids

;rotor synchronized, set cnst $31=$ spin rate, spin rate regulation

;recommended

; exchange via residual dipolar coupling or chemical exchange

; at high spin rates, spin diffusion will be slow

;phase sensitive mode is used, but sidebands will not be in phase

; this requires time reversal mode as in rfdrps.98

;written by HF, 16.9.98

; use TPPI for transform, phases will be dispersive with $n \mathrm{~s}<8$

;set:

$; \mathrm{p} 3=90$ degree $1 \mathrm{H}$ pulse

;p15 = contact pulse (typ. 1ms)

$;$ parmod $=2 \mathrm{D}$

$; \mathrm{td} 1=\mathrm{si} / 4=\mathrm{si} 1 / 2$

;nd $0=2$, in $0=\mathrm{dw}$

;cnst31 $=$ MAS spin rate

$; \mathrm{ns}=4$ for absolute value mode, $\mathrm{ns}=8$ for pure phases, $\mathrm{ns}=\mathrm{n} * 16$ with

;quadimage suppression

; set fq1 $1=\mathrm{O} 2$ frequency for on resonance decoupling, fq $2=\mathrm{LG}$ offset for

;decoupler power level p113

define loopcounter count

"count $=\mathrm{td} 1 / 2$ "

"d $31=1 \mathrm{~s} / 131 "$

\#include $<$ observe.incl $>$

\#include $<$ powswi.incl $>$

\#include $<$ trigg.incl $>$

\#include <preamp.incl $>$

$1 \mathrm{ze}$

2 d1 do:f2

10u pl1:f1

1u hgain2

10u pl2:f2

protect

trigg

p3:f2 ph1

3u:f2 ph10

(p15 ph2):f1 (p15 ph0):f2

;preselect p12 drive power for F2

;protect all preamps

1u cw:f2

;proton 90 pulse

;allow time for the phase shift on F2 ;contact pulse 
4 dw pl12:f2

lo to 4 times 10

(p1 ph3):f1

1u do:f2

$5 \mathrm{~d} 31$

lo to 5 times 11

(p1 ph5):f1

1u pl12:f2

1u cw:f2

d3:f1 ph0

$1 \mathrm{u}$ adc ph31

aq

1u hgain 1

1u do:f2

$\mathrm{rcyc}=2$

$5100 \mathrm{~m} \mathrm{wr} \# 0$ if $\# 0 \mathrm{zd}$

$1 \mathrm{~m} \mathrm{ip} 2 * 3$

$1 \mathrm{~m}$ iu 0

lo to 1 times td 1

6 exit

$$
\begin{aligned}
& \mathrm{ph} 1=+\mathrm{y} \\
& \text { ph2 }=+y \\
& \mathrm{ph} 0=+\mathrm{x} \\
& \text { ph3 }=+x+y-x-y+x-y-x+y \\
& \text { ph5 }=+x-y+x-y+x-y+x-y \\
& +\mathrm{y}+\mathrm{x}+\mathrm{y}+\mathrm{x}+\mathrm{y}+\mathrm{x}+\mathrm{y}+\mathrm{x} \\
& \text { ph10 }=+x \\
& \text { ph31=0 } 22000220
\end{aligned}
$$

;preset $10=0$

;mixing time with Gullion sequence

; set 11 for desired mixing time 


\section{A-2 Pulse Sequence: Centerband-Only Detection of Exchange (CODEX)}

;1.7) Program: RIDER (Relaxation-Induced Dipolar Exchange with Recoupling) ;Description: Selects $\mathrm{C}$ bonded to $\mathrm{N}$ (and slowly rotating segments) ;Requires: !!; Same pulse sequence as CODEX; in contrast to SPIDER, only $1 \mathrm{H}-$ $13 \mathrm{C}$ double-res. ;equipment is needed; but slow dynamics will produce artifacts. ;Reference(s): Saalwachter \& KSR, J. Magn. Reson. 145, 161-172 (2000).

;srcodex.toss

;from srcodex.ksr (no TOSS)

;from srcodex.ksr on SGI with modifications ;centerband-only detection of exchange ;Detlef's phase cycle put in (from ercodexmh.ksr, dated 5-24-2000) ; with multiple power levels on $13 \mathrm{C}(\mathrm{pl} 1 / \mathrm{pl} 11)$ and $1 \mathrm{H}(\mathrm{pl} 2 / \mathrm{pl} 12 / \mathrm{pl} 22)$ $; 1 \mathrm{H}$ at pl22 during $13 \mathrm{C} 180$ pulses

define delay cd15mp1 define delay cd $15 \mathrm{~m} 1 \mathrm{~h}$ define delay cd15mp2

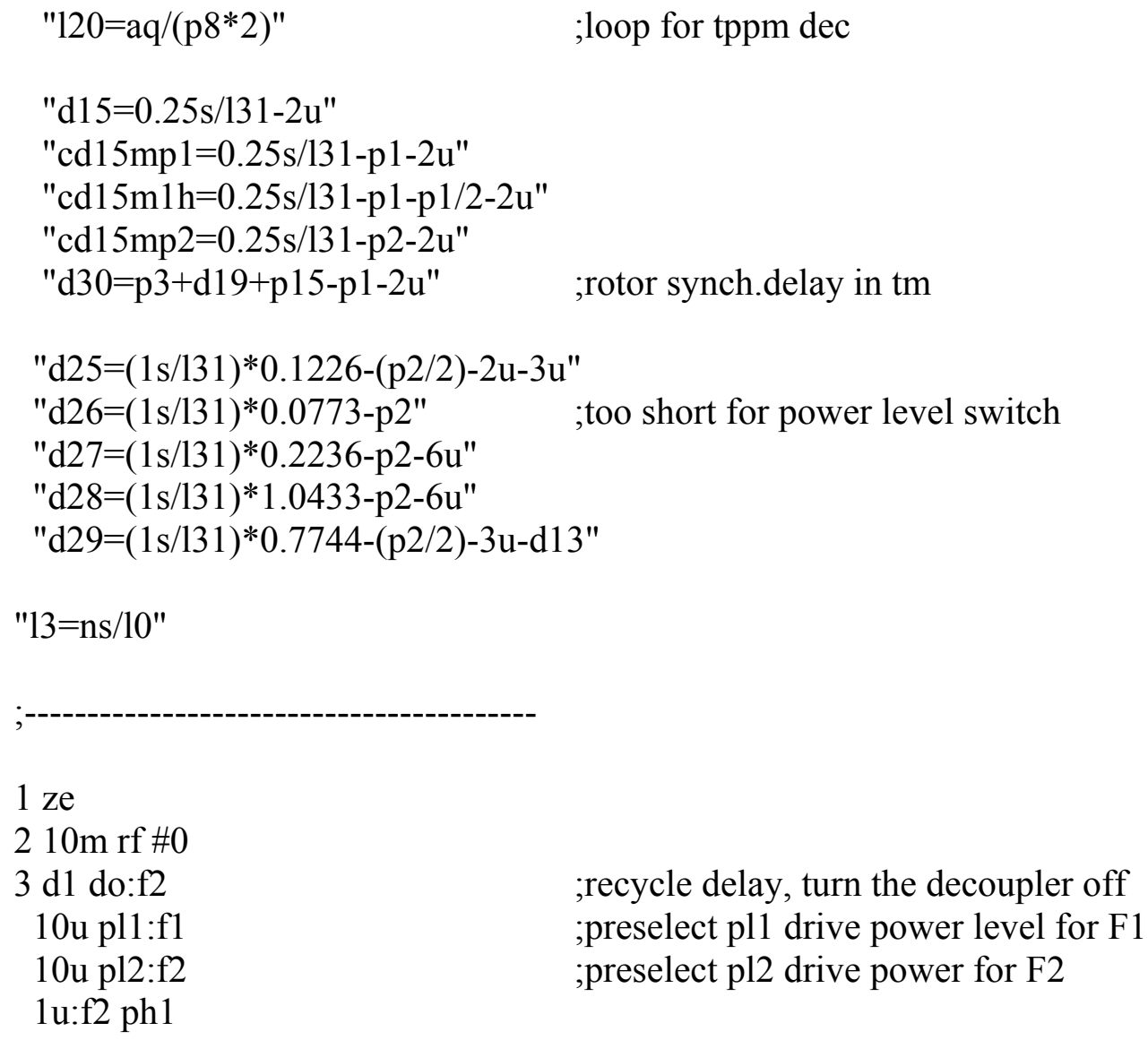

;recycle delay, turn the decoupler off ;preselect pl1 drive power level for F1 ;preselect pl2 drive power for F2 


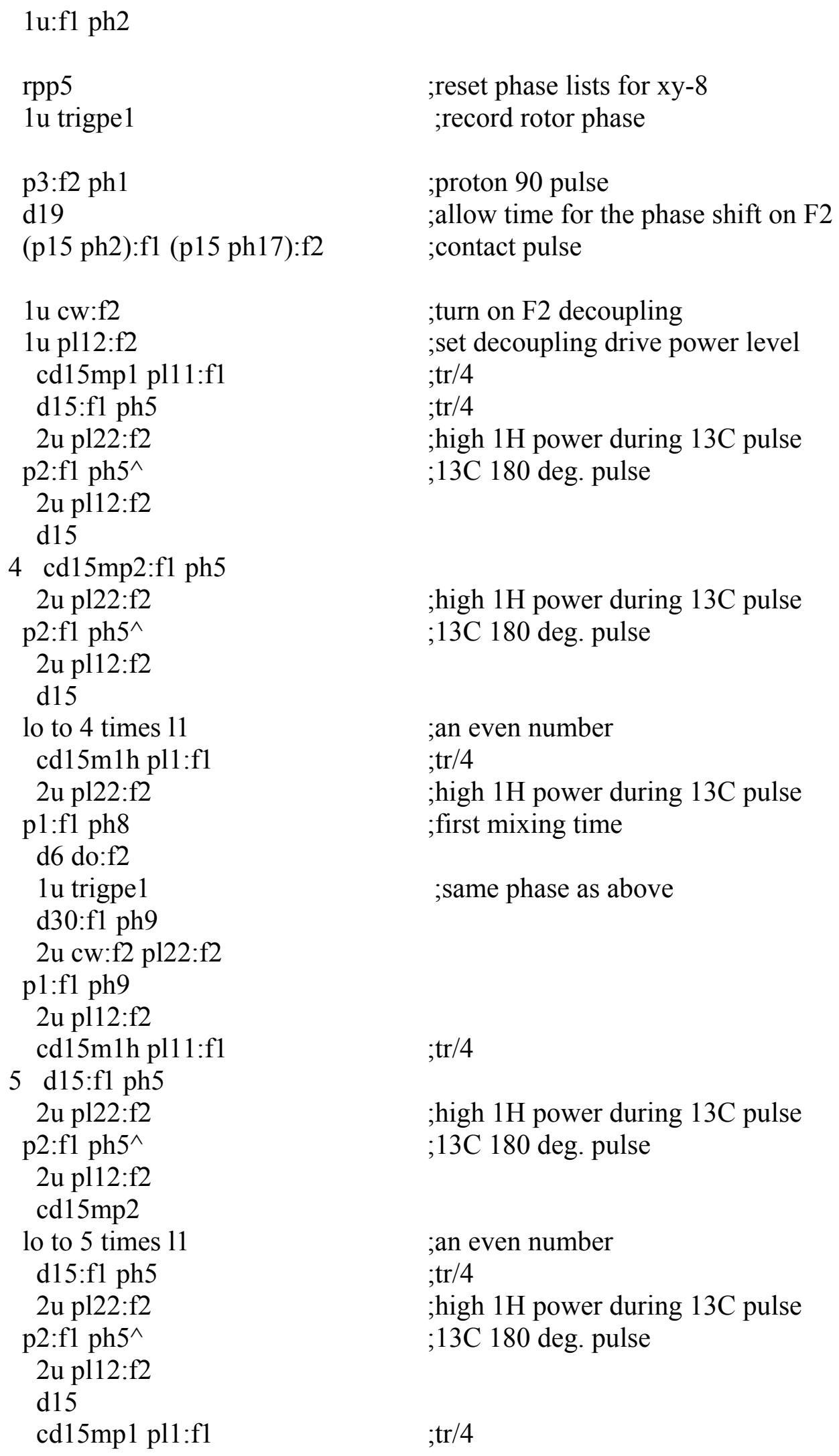

;reset phase lists for $x y-8$ ;record rotor phase

;proton 90 pulse

;allow time for the phase shift on F2 ;contact pulse

;turn on F2 decoupling ;set decoupling drive power level $; \operatorname{tr} / 4$

;rr/4

;high $1 \mathrm{H}$ power during $13 \mathrm{C}$ pulse ;13C 180 deg. pulse

;high $1 \mathrm{H}$ power during $13 \mathrm{C}$ pulse ;13C 180 deg. pulse

;an even number

;r/4

;high $1 \mathrm{H}$ power during $13 \mathrm{C}$ pulse

;first mixing time

;same phase as above

;r/4

;high $1 \mathrm{H}$ power during $13 \mathrm{C}$ pulse ;13C 180 deg. pulse

;an even number ;r $/ 4$ ;high $1 \mathrm{H}$ power during $13 \mathrm{C}$ pulse ;13C 180 deg. pulse

$; \operatorname{tr} / 4$ 


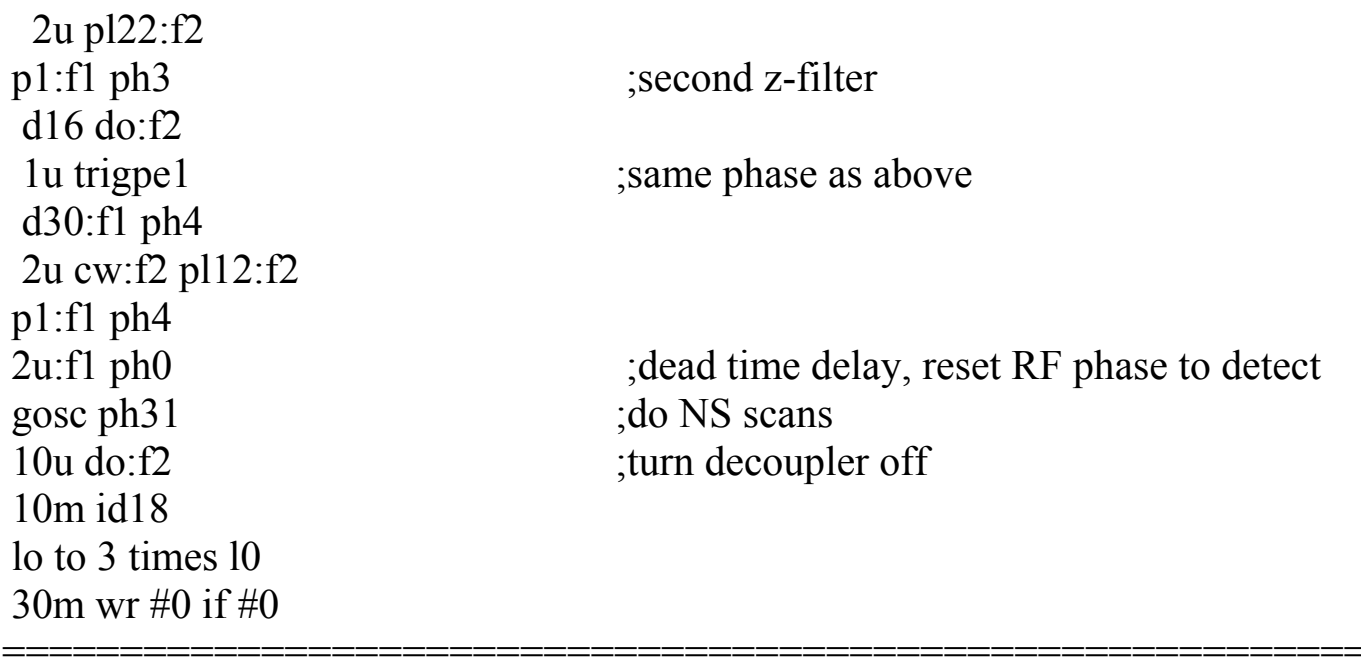

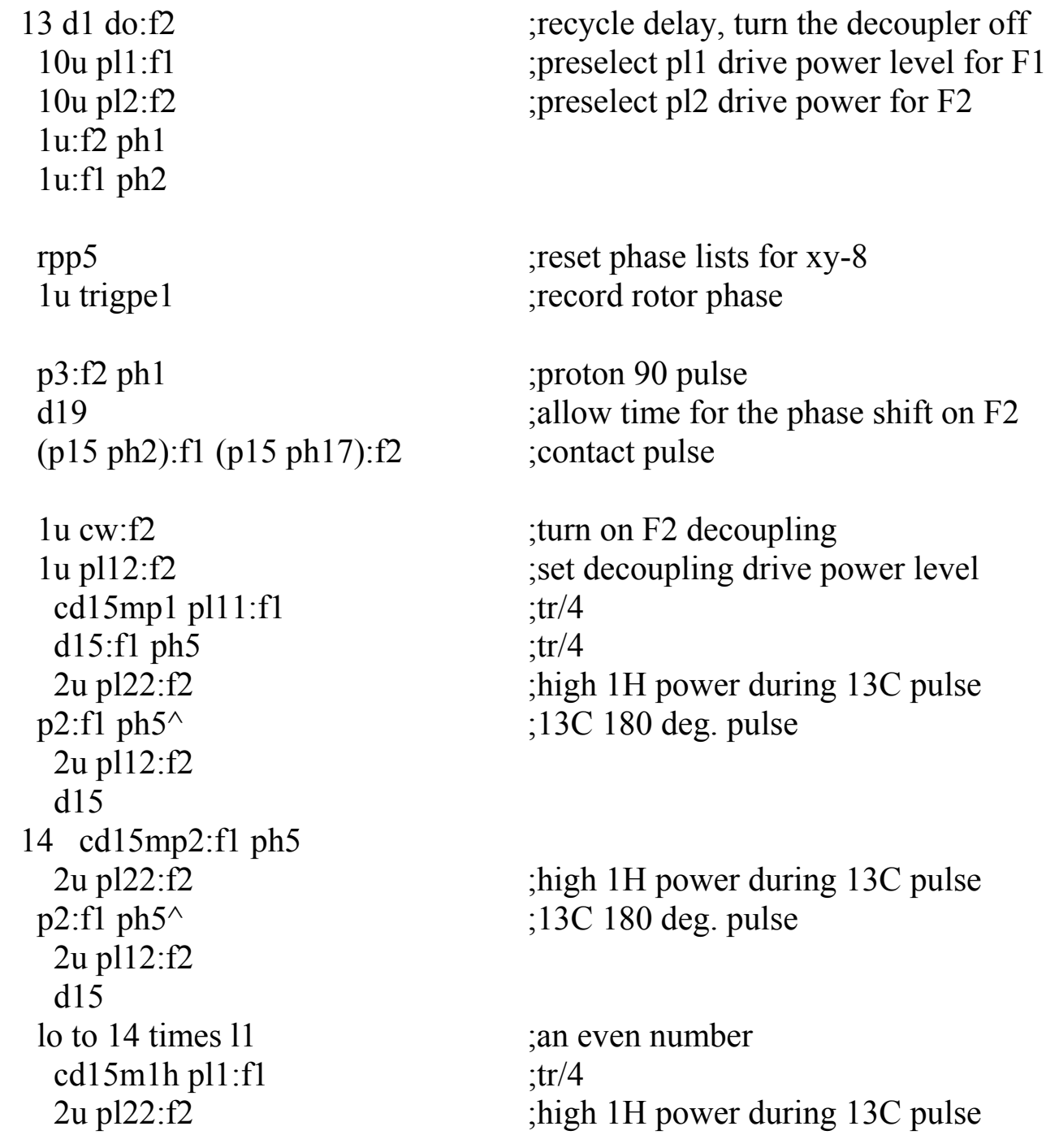




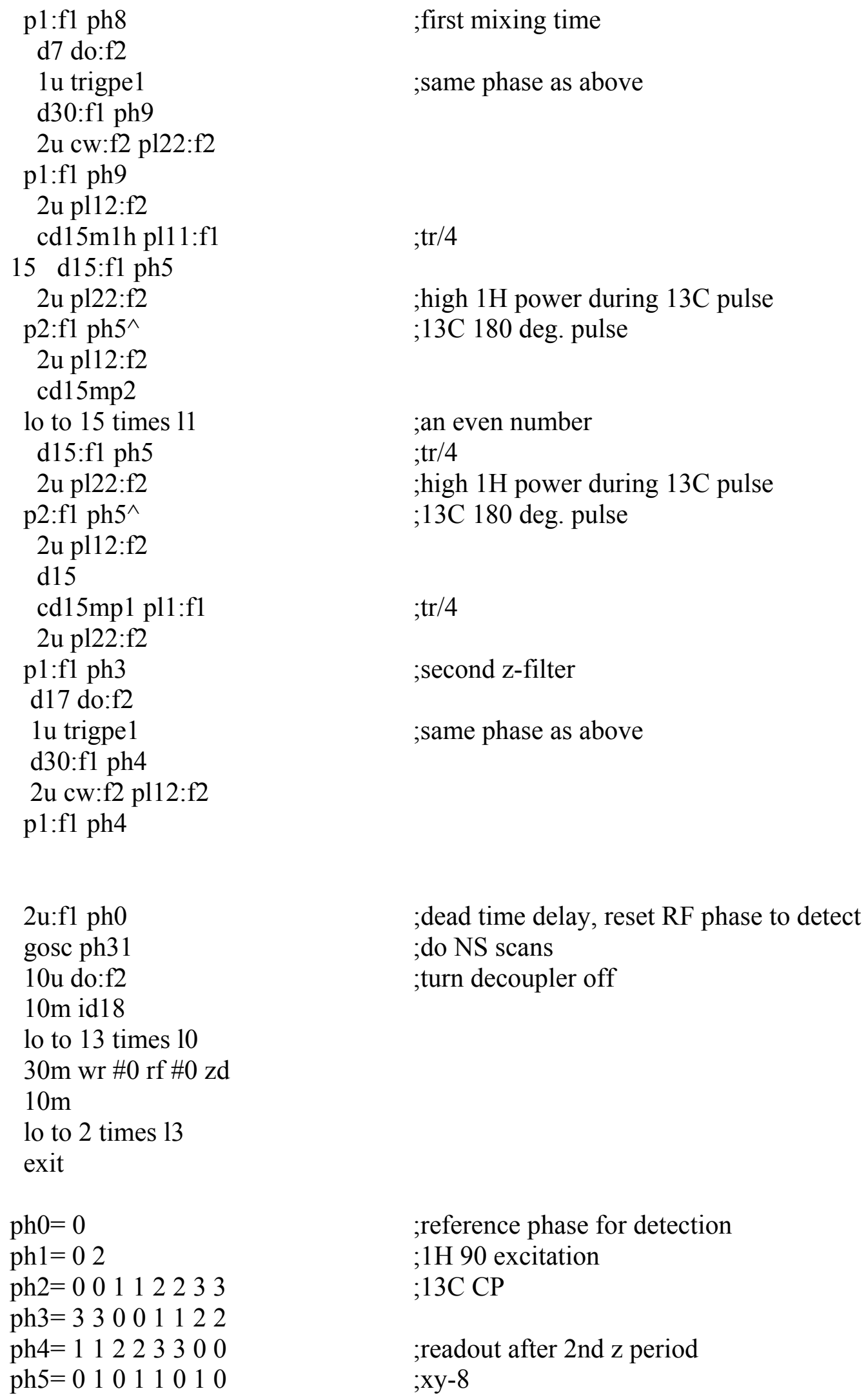

;first mixing time

;same phase as above

$; \operatorname{tr} / 4$

;high $1 \mathrm{H}$ power during $13 \mathrm{C}$ pulse

;13C 180 deg. pulse

;an even number

$; \operatorname{tr} / 4$

; high $1 \mathrm{H}$ power during $13 \mathrm{C}$ pulse

;13C 180 deg. pulse

$; \operatorname{tr} / 4$

;second z-filter

;same phase as above

; dead time delay, reset RF phase to detect ;do NS scans ;turn decoupler off

;reference phase for detection ;1H 90 excitation ;13C CP ;readout after $2 \mathrm{nd} \mathrm{z}$ period ;xy-8 


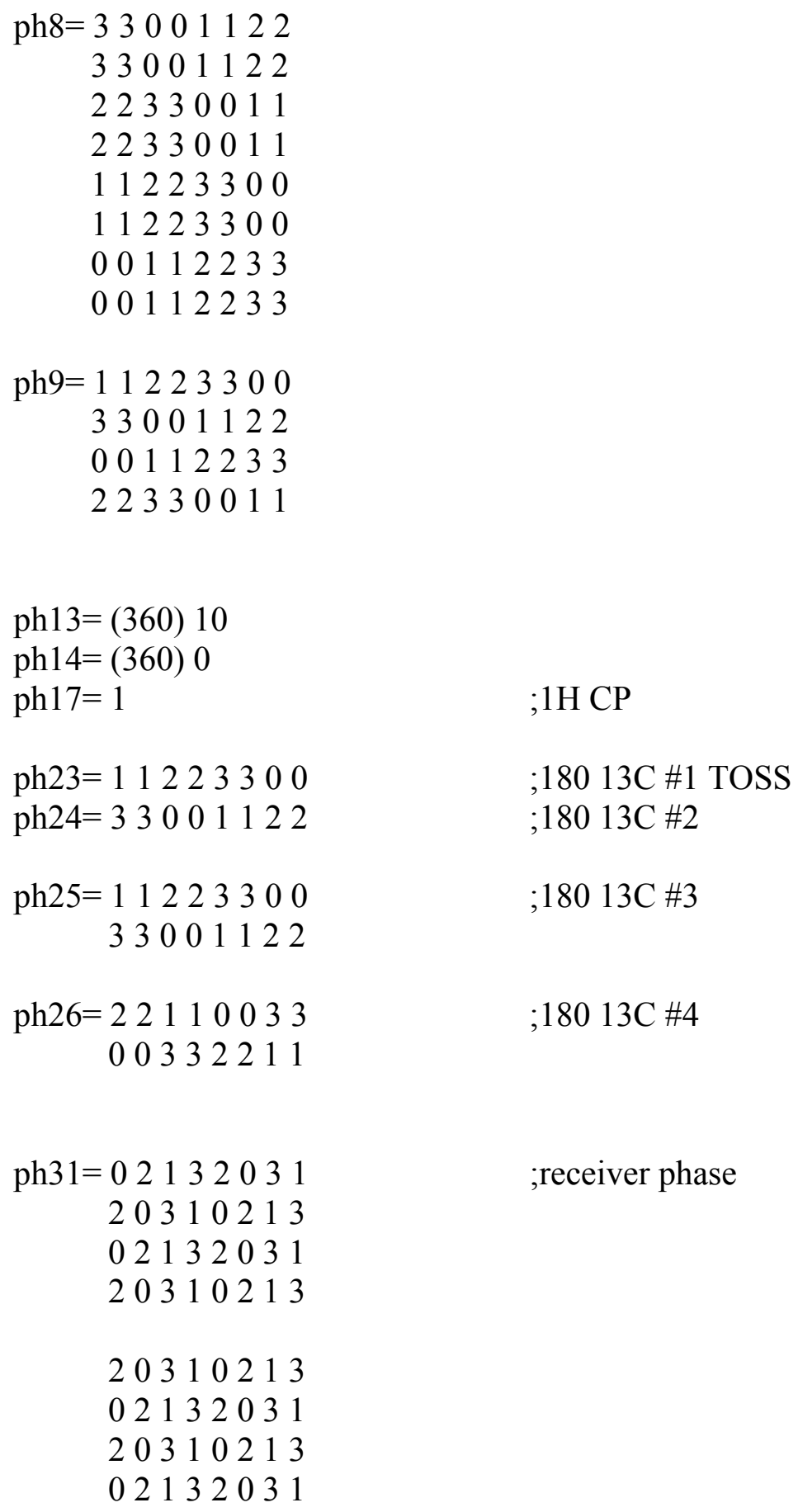




\section{A-3 Supporting Information as published (.cif file):}

\section{Bisphenol-A coordinates from $-\mathbf{6 0}^{\circ}$ single-crystal X-ray experiments}

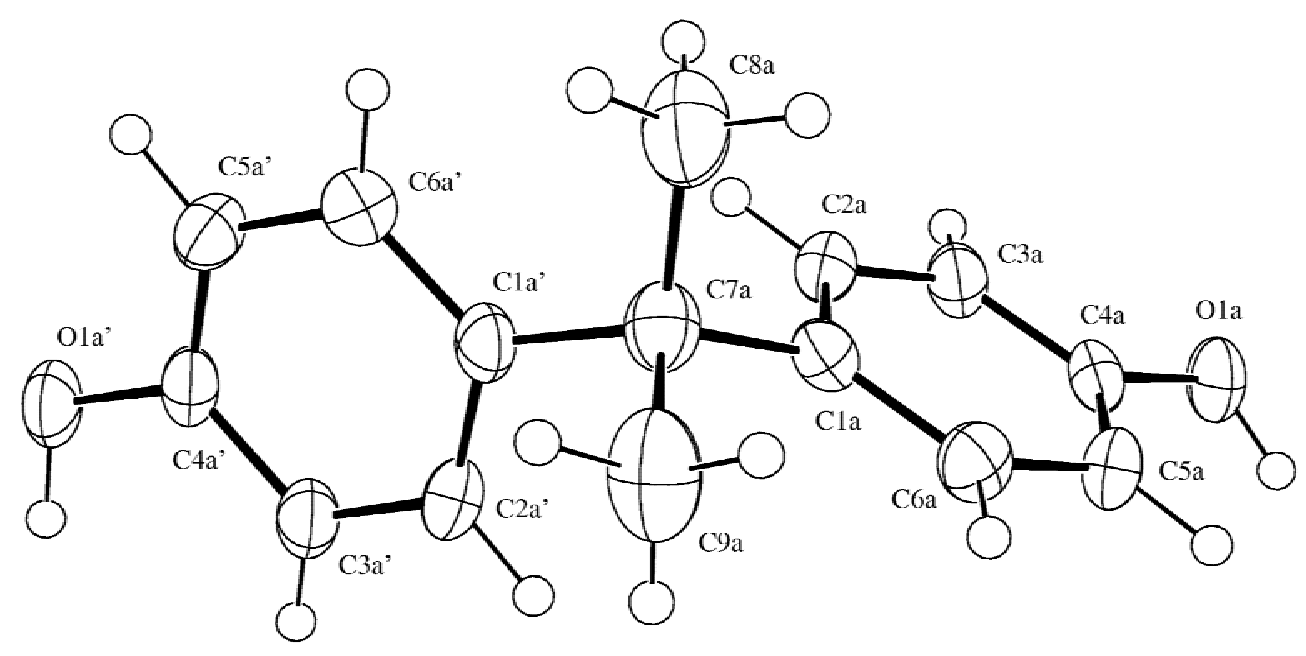

data_global

$\#==================$
$=================$

\section{\# 1. SUBMISSION DETAILS}

_publ_contact_author_name ?

publ_contact_author_address

;

? \# Enter address of contact author

;

publ_contact_author_phone?

publ_contact_author_fax ?

_publ_contact_author_email ?

_publ_requested_journal_Acta_Crystallographica_C 


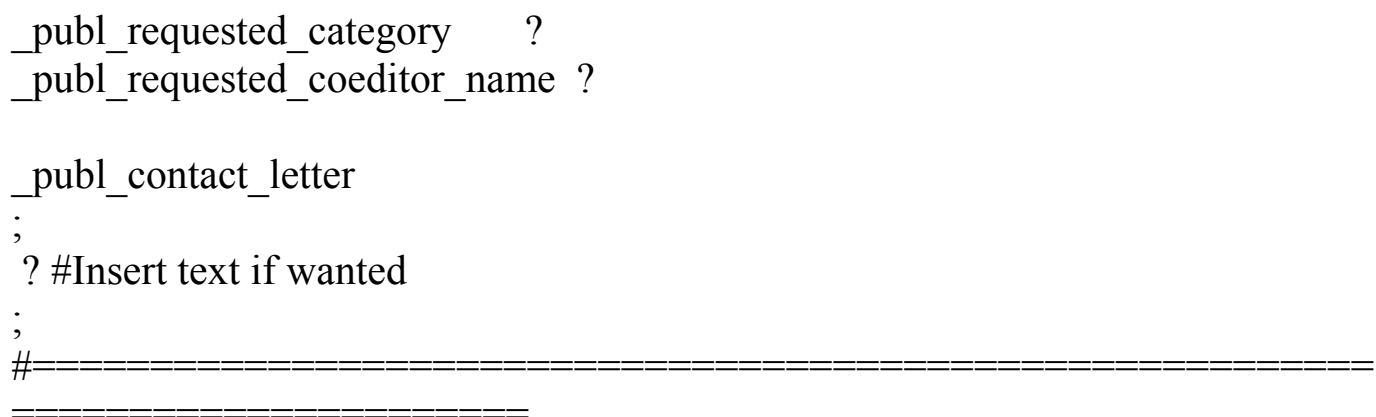

\section{\# 2. PROCESSING SUMMARY (IUCr Office Use Only)}

journal_date_recd_electronic ?

journal_date_to_coeditor?

journal_date_from_coeditor?

journal_date_accepted?

journal_date_printers_first?

journal_date_printers_final ?

journal_date_proofs_out?

journal_date_proofs_in ?

journal_coeditor_name?

journal_coeditor_code?

journal_coeditor_notes

; ?

;

journal_techeditor_code?

journal_techeditor_notes

; ?

;

journal_coden_ASTM?

journal_name_full?

journal_year ?

journal_volume?

journal_issue ?

journal_page_first ?

journal_page_last ?

journal_suppl_publ_number?

journal_suppl_publ_pages ?

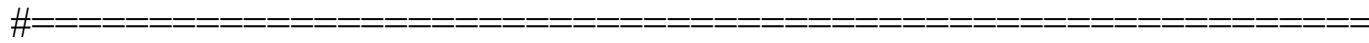

\section{\# 3. TITLE AND AUTHOR LIST}

_publ_section_title 
? \#Insert chemical title here

;

\# The loop structure below should contain the names and addresses of all

\# authors, in the required order of publication. Repeat as necessary.

loop

_publ_author_name

publ_author_address

'Boyle, Paul D.' \# \#example format

;Department of Chemistry

North Carolina State University

Raleigh, NC 27695-8204

USA

;

\section{\# 4. TEXT}

_publ_section_abstract

;

? \#Insert appropriate text here if wanted.

;

_publ_section_comment

;

? \#Insert appropriate text here if wanted.

;

_publ_section_experimental

? \#Insert appropriate text here if wanted.

;

_publ_section_references

;

Gabe, E.J., Le Page, Y., Charland,.J.-P., Lee, F.L. and White, P.S.

(1989) J. Appl. Cryst., 22, 384-387.

Flack, H., (1983) Acta Cryst., A39, 876-881.

International Tables for X-ray Crystallography, Vol. IV, (1974)

Kynoch Press, Birmingham, England.

Johnson, C.K., (1976) ORTEP - A Fortran Thermal Ellipsoid Plot

Program, Technical Report ORNL-5138, Oak Ridge National Lab., U.S.A. 
Larson, A.C., (1970) p.293, Crystallographic Computing, Munksgaard, Copenhagen.

Le Page, Y., (1988) J. Appl. Cryst., 21, 983-984.

Le Page, Y. and Gabe, E.J., (1979) J. Appl. Cryst., 12, 464-466.

Rogers, D., (1981) Acta Cryst., A37, 734-741.

? \#Insert any other references here

;

publ_section_figure_captions

;

? \#Insert figure captions here if wanted

_publ_section_exptl_prep

;

? \#Insert details of chemical and xtal preparation

_publ_section_exptl_refinement

;

? \#Special refinement details, like $\mathrm{H}$ atom treatment

publ_section_acknowledgements

The authors wish to thank the National Science Foundation (NSF)

for the funding (grant 9509532) to obtain the X-ray diffractometer.

;

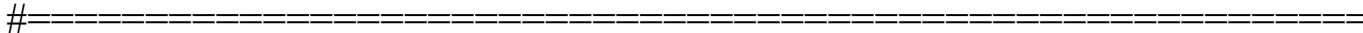

\# If more than one structure is reported, sections 5-10 should be filled in \# per structure. For each data set, replace the ? in the data_? line below \# by a unique identifier.

data_x03028

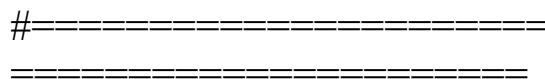

\# 5. CHEMICAL DATA

; chemical_name_systematic 
? \#Insert the chemical name here.

;

chemical name common ?

_chemical_formula_moiety 'C15 H16 O2'

_chemical_formula_structural?

_chemical_formula_analytical ?

_chemical_formula_sum 'C15 H16 O2'

chemical formula weight 228.29

_chemical_compound_source ?

loop

_atom_type_symbol

_atom_type_description

_atom_type_scat_dispersion_real

_atom_type_scat_dispersion_imag

_atom_type_scat_source

$\mathrm{C}^{-}$? $\quad{ }^{-003} \quad{ }^{-002}$ 'International Tables Vol. IV Table 2.2B'

$\mathrm{H} \quad$ ? $.000 \quad .000$ 'International Tables Vol. IV Table 2.2B'

$\mathrm{O}$ ? . 011 .006 'International Tables Vol. IV Table 2.2B'

\# 6. CRYSTAL DATA

_symmetry_cell_setting_Monoclinic

_symmetry_space_group_name_H-M 'P 21/n'

loop

_symmetry_equiv_pos_as_xyz

$\begin{array}{lc}\mathrm{x}, \mathrm{y}, \mathrm{z} & \\ -\mathrm{x},-\mathrm{y},-\mathrm{z} & \\ 1 / 2-\mathrm{x}, 1 / 2+\mathrm{y}, 1 / 2-\mathrm{z} & \\ 1 / 2+\mathrm{x}, 1 / 2-\mathrm{y}, 1 / 2+\mathrm{z} & \\ \text { cell_length_a } & 17.950(2) \\ \text {-cell_length_b } & 18.879(3) \\ \text {-cell_length_c } & 11.2281(13) \\ \text {-cel__angle_alpha } & 90.0 \\ \text {-cell_an_angle_beta } & 100.700(12) \\ \text { _cell_angle_gamma } & 90.0 \\ \text { _cell_angle_cellolume } & 3738.8(8) \\ \text {-cell_volume } & 12 \\ \text {-cell_formula_units_Z } & 12 \\ \text { _cell_measurement_temperature } & 213 \\ \text { _cell_measurement_reflns_used } & 25\end{array}$




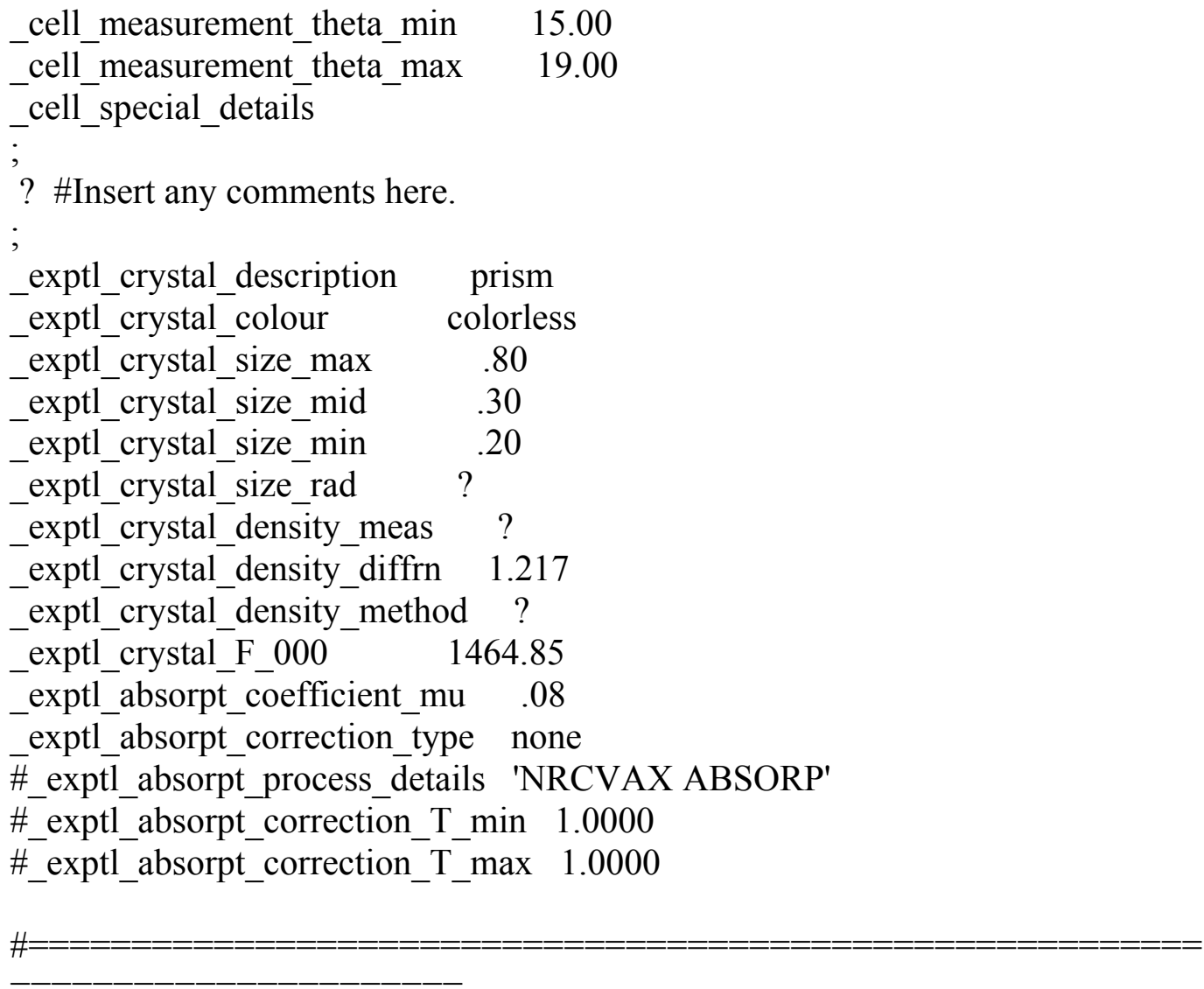

\section{\# 7. EXPERIMENTAL DATA}

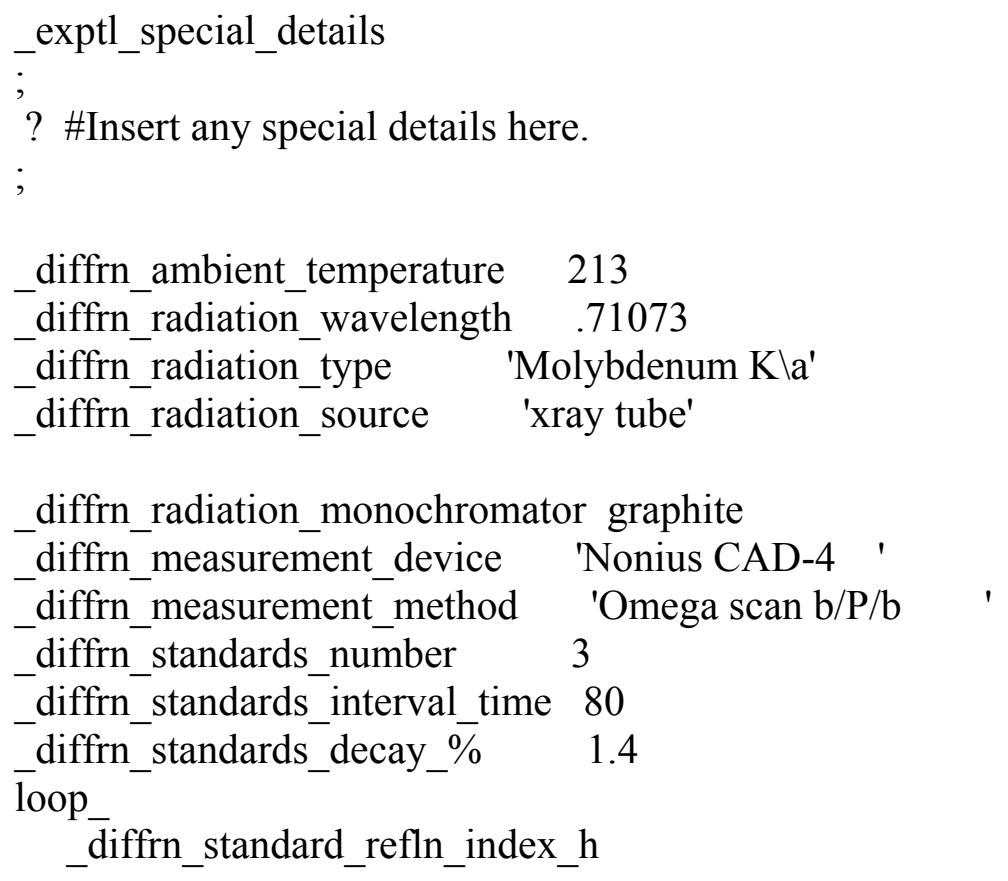


diffrn_standard_refln_index_k

diffrn_standard_refln_index_1

$\begin{array}{lll}-2 & 4 & -2\end{array}$

$\begin{array}{llll}8 & -3 & -3\end{array}$

$\begin{array}{llll}8 & -2 & -3\end{array}$

_diffrn_reflns_number 6504

_diffrn_reflns_av_R_equivalents .000

_diffrn_reflns_av_sigmaI/netI ?

_diffrn_reflns_limit_h_min -21

_diffrn_reflns_limit_h_max 20

diffrn_reflns_limit_k_min 0

diffrn_reflns_limit_k_max 22

_diffrn_reflns_limit_1_min 0

diffrn_reflns_limit__max 13

diffrn_reflns_theta_min $\quad 1.20$

diffrn reflns theta max 25.00

_diffrn_reflns_reduction_process?

_reflns_number_total 6504

reflns_number_observed 4019

_reflns_observed_criterion 'Inet $>1.0 \backslash$ s(Inet)'

_computing_data_collection 'Nonius CAD4 Argus'

_computing_cell_refinement 'Nonius CAD4 Argus'

computing_data_reduction 'NRCVAX DATRD2'

_computing_structure_solution 'SIR92'

_computing_structure_refinement 'NRCVAX LSTSQ'

_computing_molecular_graphics 'NRCVAX'

_computing_publication_material 'NRCVAX TABLES Jan 94 Version'

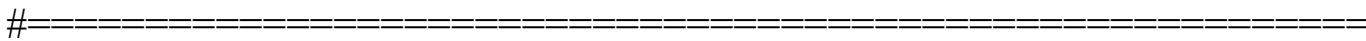

\section{\# 8. REFINEMENT DATA}

_refine_special_details

;

? \#Insert any special details here.

;

_refine_1s_structure_factor_coef $\mathrm{F}$

_refine_ls_matrix_type full

_refine_ls_weighting_scheme sigma

_refine_ls_weighting_details $\quad{ }^{\prime} 1 /\left(\backslash \mathrm{s}^{\wedge} 2^{\wedge}(\mathrm{F})+.0005 \mathrm{~F}^{\wedge} 2^{\wedge}\right)^{\prime}$ 
_refine_ls_hydrogen_treatment mixed

_refine_ls_extinction_method Larson

refine ls extinction coef $0.93(15)$

_refine_ls_number_reflns 4009

_refine_ls_number_parameters 485

_refine_ls_number_restraints ?

refine_ls_number_constraints ?

refine ls_R factor all $\quad .116$

_refine_ls_R_factor_obs $\quad .055$

_refine_ls_wR_factor_all $\quad .058$

refine_ls_wR_factor_obs $\quad .056$

refine ls goodness of fit all ?

refine ls goodness of fit obs 1.51

_refine_ls_restrained_S_all ?

_refine_ls_restrained_S_obs ?

refine_ls_shift/esd_max $\quad .000$

refine_ls_shift/esd_mean ?

_refine_diff_density_max $\quad .23$

_refine_diff_density_min $\quad-.23$

\section{\# 9. ATOMIC COORDINATES AND THERMAL PARAMETERS}

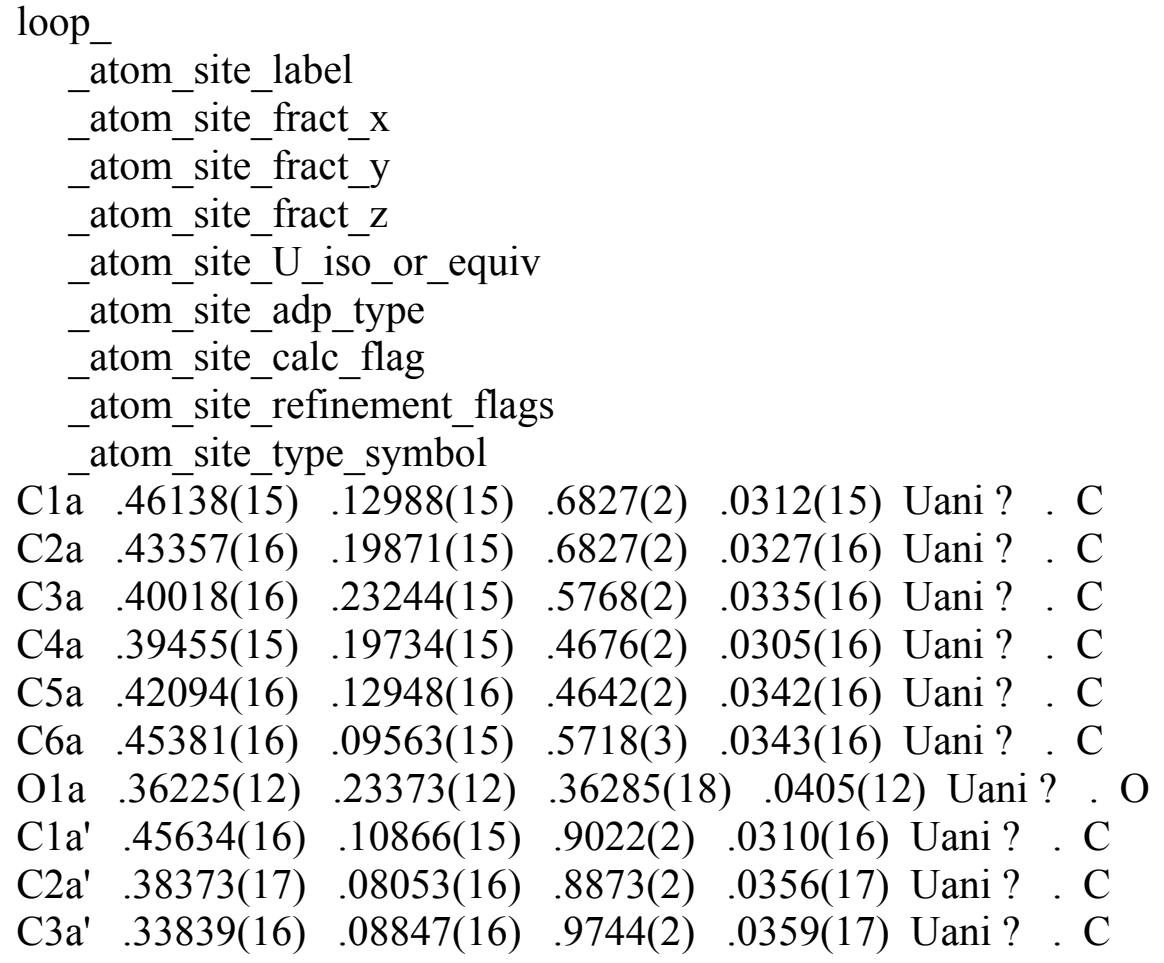




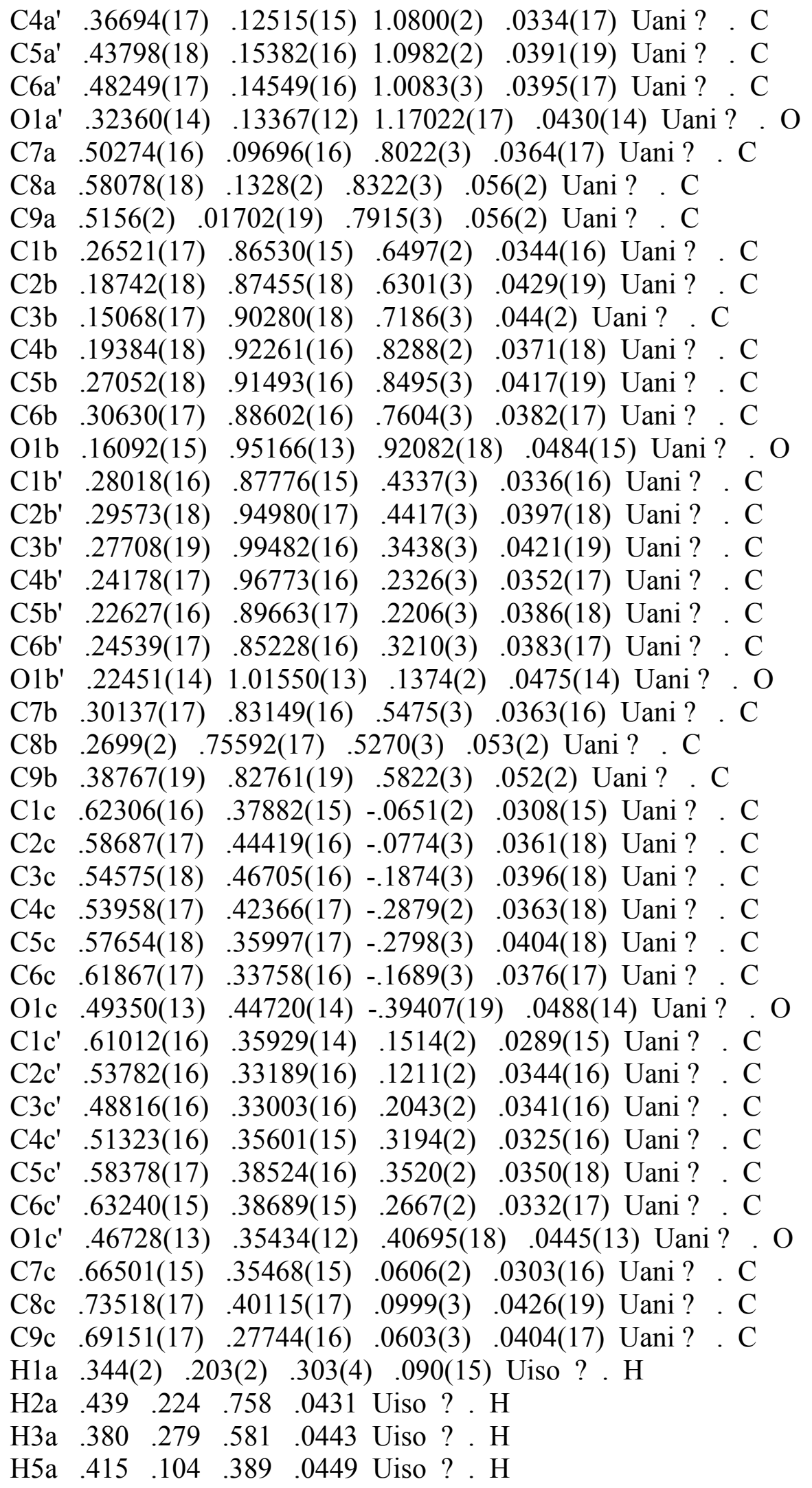


H6a $\quad .471 \quad .048 \quad .565 \quad .0451$ Uiso ? . H

H1a' .282(2) .1013(19) 1.150(3) .073(13) Uiso ? . H

$\begin{array}{llllllll}\mathrm{H} 2 \mathrm{a} & .361 & .056 & .815 & .0449 & \text { Uiso ? . H }\end{array}$

$\begin{array}{llllllll}\text { H3a' } & .289 & .068 & .959 & .0458 \text { Uiso ? . H }\end{array}$

$\begin{array}{lllllll}\mathrm{H} 5 \mathrm{a} & .460 & .180 & 1.169 & .0487 & \text { Uiso ? . } \mathrm{H}\end{array}$

$\begin{array}{lllllll}\mathrm{H}^{\prime} \mathrm{a} & .533 & .165 & 1.027 & .0494 & \text { Uiso ? . } \mathrm{H}\end{array}$

H8aa $\quad .614 \quad .113 \quad .901 \quad .0661$ Uiso ? . H

$\begin{array}{llllllll}\mathrm{H} 8 \mathrm{ab} & .575 & .183 & .844 & .0661 \text { Uiso ? . H }\end{array}$

$\begin{array}{lllllll}\mathrm{H} 8 \mathrm{ac} & .609 & .126 & .768 & .0661 \text { Uiso ? . H }\end{array}$

$\begin{array}{llllllll}\mathrm{H} 9 \mathrm{aa} & .543 & .003 & .870 & .0664 \text { Uiso ? . } \mathrm{H}\end{array}$

$\begin{array}{lllllll}\mathrm{H} 9 \mathrm{ab} & .469 & -.008 & .765 & .0664 \text { Uiso ? . H }\end{array}$

H9ac $\quad .553 \quad 008 \quad .742 \quad .0664$ Uiso ? . H

$\mathrm{H} 1 \mathrm{~b} \quad .111(2) .9548(19)$.901(3) .068(13) Uiso ? . H

$\begin{array}{llllllll}\mathrm{H} 2 \mathrm{~b} & .156 & .859 & .556 & .0527 & \text { Uiso ? . } \mathrm{H}\end{array}$

$\begin{array}{llllllll}\mathrm{H} 3 \mathrm{~b} & .097 & .913 & .698 & .0544 \text { Uiso ? . H }\end{array}$

$\begin{array}{lllllllll}\mathrm{H} 5 \mathrm{~b} & .303 & .925 & .926 & .0500 \text { Uiso ? . H }\end{array}$

$\begin{array}{lllllllll}\mathrm{H} 6 \mathrm{~b} & .360 & .880 & .781 & .0471 \text { Uiso ? . } \mathrm{H}\end{array}$

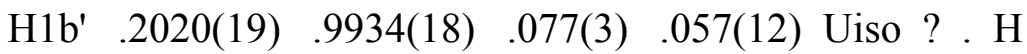

$\begin{array}{lllllll}\mathrm{H} 2 \mathrm{~b}^{\prime} & .321 & .971 & .516 & .0501 \text { Uiso ? . } \mathrm{H}\end{array}$

$\begin{array}{lllllll}\mathrm{H} 3 \mathrm{~b}^{\prime} & .286 & 1.045 & .350 & .0532 & \text { Uiso ? . } \mathrm{H}\end{array}$

$\begin{array}{llllllll}\mathrm{H} 5 \mathrm{~b}^{\prime} & .201 & .876 & .146 & .0489 & \text { Uiso ? . } \mathrm{H}\end{array}$

$\begin{array}{lllllll}\mathrm{H}^{6} \mathrm{~b}^{\prime} & .231 & .803 & .312 & .0485 & \text { Uiso ? . } \mathrm{H}\end{array}$

$\begin{array}{llllllll}\mathrm{H} 8 \mathrm{ba} & .216 & .760 & .497 & .0625 \text { Uiso ? . H }\end{array}$

$\begin{array}{lllllll}\mathrm{H} 8 \mathrm{bb} & .294 & .731 & .470 & .0625 & \text { Uiso ? . H }\end{array}$

$\begin{array}{llllllll}\mathrm{H} 8 \mathrm{bc} & .287 & .731 & .601 & .0625 & \text { Uiso ? . } \mathrm{H}\end{array}$

H9ba $\quad .405 \quad .797 \quad .650 \quad .0621$ Uiso ? . H

$\begin{array}{lllllll}\mathrm{H} 9 \mathrm{bb} & .406 & .803 & .518 & .0621 \text { Uiso ? . } \mathrm{H}\end{array}$

$\begin{array}{llllllll}\mathrm{H} 9 \mathrm{bc} & .410 & .873 & .603 & .0621 & \text { Uiso ? . } \mathrm{H}\end{array}$

H1c .4917(19) $.4119(17)-.449(3)$.061(11) Uiso ? . H

$\begin{array}{llllllll}\mathrm{H} 2 \mathrm{c} & .590 & .474 & -.007 & .0468 & \text { Uiso ? . } \mathrm{H}\end{array}$

$\begin{array}{llllllll}\mathrm{H} 3 \mathrm{c} & .517 & .510 & -.194 & .0502 & \text { Uiso ? . } \mathrm{H}\end{array}$

$\begin{array}{llllllll}\mathrm{H} 5 \mathrm{c} & .572 & .329 & -.348 & .0516 & \text { Uiso ? . } \mathrm{H}\end{array}$

$\mathrm{H} 6 \mathrm{c} \quad .645 \quad .293-.168$.0486 Uiso ? . H

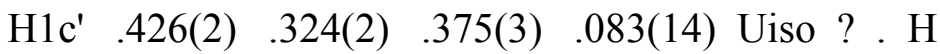

$\begin{array}{lllllll}\mathrm{H} 2 \mathrm{c}^{\prime} & .519 & .312 & .043 & .0448 \text { Uiso ? . H }\end{array}$

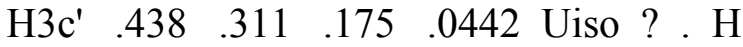

$\begin{array}{lllllll}\mathrm{H} 5 \mathrm{c}^{\prime} & .602 & .403 & .432 & .0453 & \text { Uiso ? . } \mathrm{H}\end{array}$

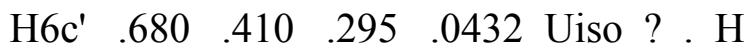

$\begin{array}{lllllll}\mathrm{H} 8 \mathrm{ca} & .721 & .449 & .110 & .0532 & \text { Uiso ? . } \mathrm{H}\end{array}$

$\begin{array}{llllllll}\mathrm{H} 8 \mathrm{cb} & .767 & .387 & .174 & .0532 & \text { Uiso ? . H }\end{array}$

$\begin{array}{lllllll}\mathrm{H} 8 \mathrm{cc} & .766 & .396 & .039 & .0532 & \text { Uiso ? . H }\end{array}$

$\begin{array}{lllllll}\mathrm{H} 9 \mathrm{ca} & .719 & .264 & .138 & .0507 & \text { Uiso ? . H }\end{array}$

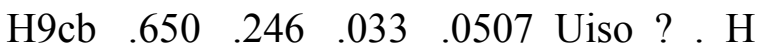

$\begin{array}{lllllllll}\mathrm{H} 9 \mathrm{cc} & .729 & .268 & .011 & .0507 & \text { Uiso ? . H }\end{array}$ 
loop

_atom_site_aniso_label

atom site aniso U 11

_atom_site_aniso_U_22

_atom_site_aniso_U_33

_atom_site_aniso_U_12

_atom_site_aniso_U_13

atom_site_aniso_U_23

_atom_site_aniso_type_symbol

C1a .0317(17).0332(18).0305(16) -.0031(14).0106(13).0033(13) C

C2a .0399(18).0342(18).0252(15) -.0030(14).0092(13) -.0020(13) C

C3a .0425(18).0317(17).0286(16).0018(14).0127(14).0027(13) C

C4a .0317(17).0366(18).0253(16) -.0006(14).0110(13).0063(13) C

C5a .0393(18).0409(19).0245(16).0030(15).0112(13) -.0023(13) C

C6a.0372(18).0322(17).0358(17).0043(14).0127(14) -.0029(14) C

O1a .0510(14).0475(14).0235(12).0036(11).0079(10).0085(11) O

C1a'.0380(17).0323(17).0221(15).0018(14).0041(13).0069(13) C

C2a' .0415(19).0434(19).0199(15).0003(15).0005(13).0024(13) C

C3a'.0356(18).0469(19).0249(16) -.0025(15).0047(13).0049(14) C

C4a' .0435(19).0361(18).0214(15).0035(15).0078(13).0041(13) C

C5a'.051(2) .0375(19).0273(17) -.0044(16) .0037(15) -.0039(14) C

C6a'.0437(20).0407(19).0337(18) -.0079(15) .0057(15).0031(14) C

O1a' .0597(16).0490(14).0232(11) -.0003(13).0151(11).0014(10) O

C7a .0349(18).0436(19).0304(17).0058(15).0052(13).0045(14) C

C8a .0350(19).093(3).0401(20).0041(19).0053(15).0108(19) C

C9a .069(2).053(2).047(2) .0297(19).0164(18).0143(17) C

C1b .0392(19).0326(17).0316(17) -.0028(14).0072(14).0060(13) C

C2b .0399(20).060(2).0282(17) -.0078(17).0045(14) -.0061(15) C

C3b .0368(18).068(2).0287(17) -.0028(17).0063(14) -.0010(16) C

C4b .049(2) .0404(19).0229(16) -.0033(15).0081(14).0046(14) C

C5b .048(2) .044(2).0276(17) .0020(16) -.0062(14).0002(15) C

C6b .0374(18).0399(18).0339(18).0004(15) -.0019(14).0038(15) C

O1b .0543(17).0654(16).0258(12).0040(13).0087(11) -.0030(11) O

C1b'.0331(17).0351(18).0356(18) -.0031(14).0147(13) -.0056(14) C C2b' .053(2) .0400(19) .0269(17) -.0072(16) .0103(14) -.0054(14) C

C3b' .063(2).0358(18).0309(18) -.0067(16) .0174(16) -.0044(14) C C4b'.0391(18).0433(20).0279(17) -.0008(15) .0180(14) .0007(14) C C5b'.0388(18).048(2) .0296(17) -.0051(15) .0090(14) -.0082(15) C

C6b'.0370(18).0384(18) .0400(18) -.0057(15).0081(14) -.0062(15) C O1b'.0621(16).0545(15).0280(13) -.0076(13).0139(11).0019(12) O C7b .0387(18) .0359(18).0345(17).0024(15) .0070(14) -.0022(14) C C8b .069(2).038(2).051(2).0046(18).0083(18).0039(16) C C9b .048(2).060(2) .048(2).0095(18).0073(16) -.0063(18) C C1c .0335(17).0354(17).0267(16) -.0043(14).0142(13).0031(13) C C2c .0435(19).0366(18).0304(17) -.0002(15) .0121(14) -.0012(14) C 
C3c .0485(20).0385(19).0337(18).0052(15).0125(15).0043(14) C C4c .0404(18).047(2).0237(16) -.0010(15) .0111(14) .0047(14) C C5c .049(2) .048(2).0285(17).0025(16).0172(15) -.0035(15) C

C6c .0461(19).0383(18).0314(17).0039(15).0145(14).0016(14) C O1c .0582(15).0675(16).0218(12).0115(13).0100(11).0036(12) O C1 ' .0322(17).0302(16).0240(15) -.0011(13).0051(12).0047(13) C C2c'.0368(18).0446(19).0231(15) -.0063(15).0088(13) -.0015(13) C C3c'.0328(17).0406(18).0292(16) -.0074(14).0070(13).0022(14) C C4c'.0383(18).0335(17).0292(16) -.0002(14).0150(14) .0042(13) C C5c'.0464(19).0391(18).0203(15) -.0016(15).0079(13) -.0025(13) C C6c'.0291(16).0400(18).0304(17) -.0073(14).0055(13) -.0003(13) C O1c'.0536(15).0554(15).0310(12) -.0088(12).0244(11) -.0010(11) O C7c .0306(16).0369(17).0259(15) -.0005(13).0120(13).0011(13) C C8c .0365(19).056(2).0372(18) -.0044(16) .0114(14) .0019(15) C C9c .0400(18).047(2).0351(17).0088(15).0094(14).0063(15) C

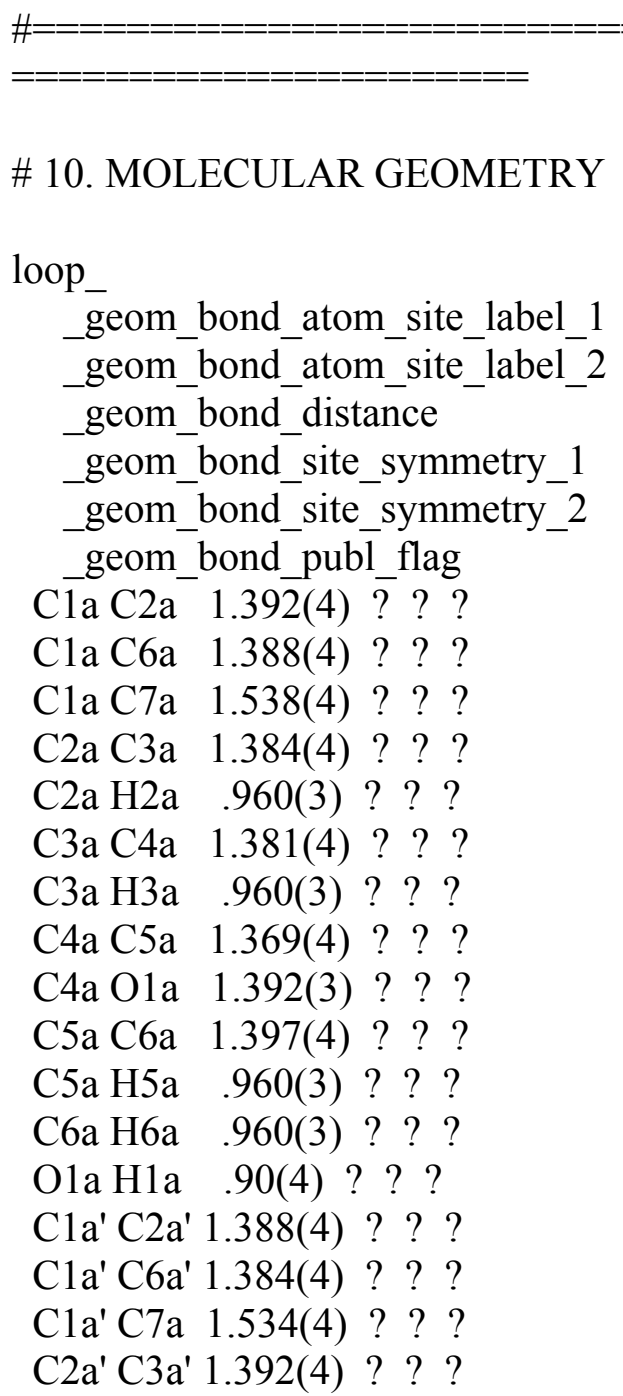


C2a' H2a' .960(3) ? ? ?

C3a' C4a' 1.386(4) ? ? ?

C3a' H3a' .960(3) ? ? ?

C4a' C5a' 1.365(4) ? ? ?

C4a' O1a' 1.397(3) ? ? ?

C5a' C6a' 1.407(4) ? ? ?

C5a' H5a' .960(3) ? ? ?

C6a' H6a' .960(3) ? ? ?

O1a' H1a' .96(4) ? ? ?

$\mathrm{C} 7 \mathrm{a} \mathrm{C} 8 \mathrm{a}$ 1.536(5) ? ? ?

$\mathrm{C} 7 \mathrm{a} \mathrm{C9a} 1.535(5)$ ? ? ?

C8a H8aa .960(3) ? ?

$\mathrm{C} 8 \mathrm{a} \mathrm{H} 8 \mathrm{ab}$.960(4) ? ? ?

C8a H8ac .960(3) ? ?

C9a H9aа .960(3) ? ? ?

C9a H9ab .960(4) ? ? ?

C9a H9ac .960(3) ? ? ?

$\mathrm{C} 1 \mathrm{~b} \mathrm{C} 2 \mathrm{~b}$ 1.384(4) ? ?

C1b C6b 1.380(4) ? ?

$\mathrm{C} 1 \mathrm{~b} \mathrm{C} 7 \mathrm{~b}$ 1.556(4) ? ?

$\mathrm{C} 2 \mathrm{~b} \mathrm{C} 3 \mathrm{~b}$ 1.396(4) ? ?

$\mathrm{C} 2 \mathrm{~b} \mathrm{H} 2 \mathrm{~b}$.960(3) ? ?

$\mathrm{C} 3 \mathrm{~b} \mathrm{C} 4 \mathrm{~b}$ 1.383(4) ? ?

$\mathrm{C} 3 \mathrm{~b} \mathrm{H} 3 \mathrm{~b}$.960(3) ? ?

$\mathrm{C} 4 \mathrm{~b}$ C5b 1.360(5) ? ?

$\mathrm{C} 4 \mathrm{~b} \mathrm{O} 1 \mathrm{~b}$ 1.394(4) ? ?

C5b C6b 1.396(4) ? ?

$\mathrm{C} 5 \mathrm{~b} \mathrm{H} 5 \mathrm{~b}$.960(3) ? ?

C6b H6b .960(3) ? ?

O1b H1b .89(4) ? ? ?

C1b' C2b' 1.388(4) ? ?

C1b' C6b' 1.389(4) ? ? ?

C1b' C7b 1.537(4) ? ?

C2b' C3b' 1.381(4) ? ? ?

C2b' H2b' .960(3) ? ? ?

C3b' C4b' 1.389(4) ? ? ?

C3b' H3b' .960(3) ? ? ?

C4b' C5b' 1.372(4) ? ? ?

C4b' O1b' 1.388(4) ? ?

C5b' C6b' 1.395(4) ? ? ?

C5b' H5b' .960(3) ? ? ?

C6b' H6b' .960(3) ? ? ?

O1b' H1b' .84(3) ? ? ?

$\mathrm{C} 7 \mathrm{~b}$ C8b 1.536(4) ? ?

$\mathrm{C} 7 \mathrm{~b} \mathrm{C9b} 1.527(4)$ ? ? 
C8b H8ba .960(4) ? ? ?

C8b H8bb .960(3) ? ?

$\mathrm{C} 8 \mathrm{~b} \mathrm{H} 8 \mathrm{bc}$.960(3) ? ?

C9b H9ba .960(3) ? ? ?

C9b H9bb .960(3) ? ? ?

C9b H9bc .960(4) ? ?

$\mathrm{C} 1 \mathrm{c} \mathrm{C} 2 \mathrm{c}$ 1.390(4) ? ?

$\mathrm{C} 1 \mathrm{c}$ C6c 1.391(4) ? ?

$\mathrm{C} 1 \mathrm{c} \mathrm{C} 7 \mathrm{c} 1.540(4)$ ? ? ?

$\mathrm{C} 2 \mathrm{c} \mathrm{C} 3 \mathrm{c}$ 1.384(4) ? ?

$\mathrm{C} 2 \mathrm{c} \mathrm{H} 2 \mathrm{c}$.960(3) ? ?

$\mathrm{C} 3 \mathrm{c} \mathrm{C} 4 \mathrm{c}$ 1.382(4) ? ?

$\mathrm{C} 3 \mathrm{c} \mathrm{H} 3 \mathrm{c}$.960(3) ? ?

$\mathrm{C} 4 \mathrm{c}$ C5c 1.368(4) ? ?

$\mathrm{C} 4 \mathrm{c} \mathrm{O} 1 \mathrm{c}$ 1.392(3) ? ?

$\mathrm{C} 5 \mathrm{c}$ C6c 1.397(4) ? ? ?

$\mathrm{C} 5 \mathrm{c} \mathrm{H} 5 \mathrm{c}$.960(3) ? ?

C6c H6c .960(3) ? ? ?

$\mathrm{O} 1 \mathrm{cH} \mathrm{H}$.90(3) ? ? ?

$\mathrm{C} 1 \mathrm{c}^{\prime} \mathrm{C} 2 \mathrm{c}^{\prime} 1.380(4)$ ? ? ?

$\mathrm{C} 1 \mathrm{c}^{\prime} \mathrm{C} 6 \mathrm{c}^{\prime} 1.384(4)$ ? ? ?

$\mathrm{C} 1 \mathrm{c}^{\prime} \mathrm{C} 7 \mathrm{c}$ 1.547(4) ? ? ?

$\mathrm{C} 2 \mathrm{c}^{\prime} \mathrm{C} 3 \mathrm{c}^{\prime} 1.406(4)$ ? ? ?

$\mathrm{C} 2 \mathrm{c}^{\prime} \mathrm{H} 2 \mathrm{c}^{\prime}$.960(3) ? ? ?

$\mathrm{C} 3 \mathrm{c}^{\prime} \mathrm{C} 4 \mathrm{c}^{\prime} 1.378(4)$ ? ? ?

$\mathrm{C} 3 \mathrm{c}^{\prime} \mathrm{H} 3 \mathrm{c}^{\prime}$.960(3) ? ? ?

$\mathrm{C} 4 c^{\prime} \mathrm{C} 5 c^{\prime} 1.368(4)$ ? ? ?

$\mathrm{C} 4 \mathrm{c}^{\prime} \mathrm{O} 1 \mathrm{c}^{\prime} 1.396(3)$ ? ? ?

C5c' C6c' 1.411(4) ? ? ?

C5c' H5c' .960(3) ? ? ?

C6c' H6c' .960(3) ? ? ?

O1c' H1c' .95(4) ? ? ?

$\mathrm{C} 7 \mathrm{c} \mathrm{C} 8 \mathrm{c} 1.531(4)$ ? ? ?

$\mathrm{C} 7 \mathrm{c}$ C9c 1.534(4) ? ? ?

$\mathrm{C} 8 \mathrm{c} \mathrm{H} 8 \mathrm{ca}$.960(3) ? ?

$\mathrm{C} 8 \mathrm{c} \mathrm{H} 8 \mathrm{cb}$.960(3) ? ?

$\mathrm{C} 8 \mathrm{c} \mathrm{H} 8 \mathrm{cc} .960(3)$ ? ? ?

C9c H9ca .960(3) ? ? ?

C9c H9cb .960(3) ? ? ?

C9c H9cc .960(3) ? ? ?

H8aa H8ac 1.49493(17) ? ? ?

H9aa H9ac 1.48619(17) ? ? ?

H8bb H8bc 1.50449(17) ? ?

H9ba H9bb 1.49334(17) ? ?

H9ba H9bc 1.5331(2) ? ? 
H8ca H8cb 1.54676(17)？？ $\mathrm{H} 8 \mathrm{cb} \mathrm{H} 8 \mathrm{cc} 1.52118(17)$ ? ? ? H9ca H9cc 1.47583(17) ? ? ? H9cb H9cc 1.53726(16)？？

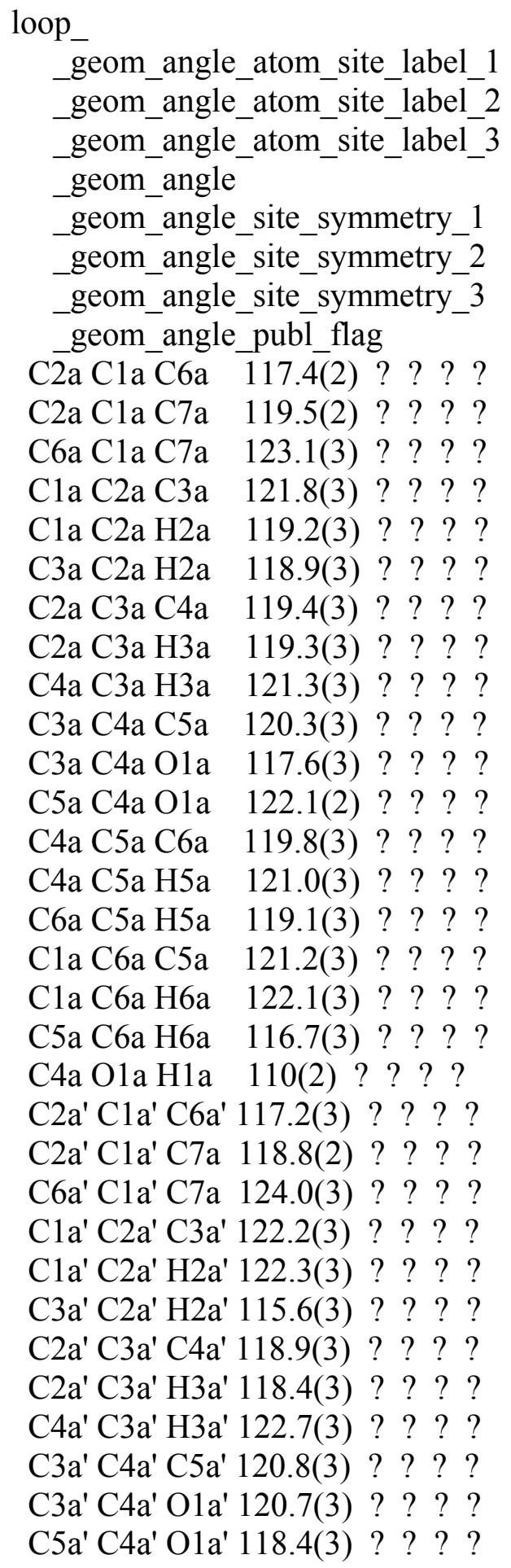


C4a' C5a' C6a' 119.2(3) ?? ? ?

C4a' C5a' H5a' 123.9(3) ?? ? ?

C6a' C5a' H5a' 117.0(3) ? ? ? ?

C1a' C6a' C5a' 121.8(3) ?? ? ?

C1a' C6a' H6a' 122.2(3) ? ? ? ?

C5a' C6a' H6a' 116.0(3) ? ? ? ?

C4a' Ola' H1a' 105(2) ? ? ? ?

Cla C7a C1a' 109.9(2) ? ? ? ?

$\mathrm{Cla}$ C7a C8a 106.6(2) ?? ? ?

$\mathrm{C} 1 \mathrm{a} \mathrm{C7aC9a} 112.6(2)$ ? ? ? ?

Cla' C7a C8a 111.9(2) ?? ? ?

Cla' C7a C9a 108.0(2) ?? ? ?

$\mathrm{C} 8 \mathrm{aC} 7 \mathrm{aC} 9 \mathrm{a}$ 107.9(3) ?? ? ?

C7a C8a H8aa 114.6(3) ? ? ? ?

$\mathrm{C7a}$ C8a H8ab 109.9(3) ?? ? ?

$\mathrm{C} 7 \mathrm{a}$ C8a H8ac 111.0(3) ?? ? ?

H8aa C8a H8ab 109.6(3) ? ? ? ?

H8aa C8a H8ac 102.3(3) ? ? ? ?

$\mathrm{H} 8 \mathrm{ab}$ C8a H8ac 109.2(3) ? ? ? ?

C7a C9a H9aa 105.0(3) ? ? ? ?

$\mathrm{C} 7 \mathrm{a} \mathrm{C} 9 \mathrm{a} \mathrm{H} 9 \mathrm{ab}$ 112.3(3) ?? ? ?

$\mathrm{C7aC9aH9ac} \mathrm{110.7(3)} \mathrm{?} \mathrm{?} \mathrm{?} \mathrm{?}$

H9aa C9a H9ab 113.7(4)？？？

H9aa C9a H9ac 101.4(3) ? ? ? ?

$\mathrm{H} 9 \mathrm{ab}$ C9a H9ac 113.0(4) ? ? ? ?

$\mathrm{C} 2 \mathrm{~b} \mathrm{C1b} \mathrm{C6b} \mathrm{117.5(3)} \mathrm{??} \mathrm{?} \mathrm{?}$

$\mathrm{C} 2 \mathrm{~b} \mathrm{C1b} \mathrm{C7b} \mathrm{118.8(2)} \mathrm{???} \mathrm{?}$

$\mathrm{C} 6 \mathrm{~b} \mathrm{C1b} \mathrm{C7b} \mathrm{123.7(3)} \mathrm{??} \mathrm{?} \mathrm{?}$

$\mathrm{C} 1 \mathrm{~b}$ C2b C3b 122.1(3) ?? ? ?

$\mathrm{C} 1 \mathrm{~b} \mathrm{C} 2 \mathrm{~b} \mathrm{H} 2 \mathrm{~b}$ 121.4(3) ?? ? ?

$\mathrm{C} 3 \mathrm{~b} \mathrm{C} 2 \mathrm{~b} \mathrm{H} 2 \mathrm{~b}$ 116.4(3) ?? ? ?

$\mathrm{C} 2 \mathrm{~b} \mathrm{C} 3 \mathrm{~b} \mathrm{C} 4 \mathrm{~b}$ 118.6(3) ?? ? ?

$\mathrm{C} 2 \mathrm{~b}$ C3b H3b 119.4(3) ? ? ?

$\mathrm{C} 4 \mathrm{~b}$ C3b H3b 121.6(3) ? ? ? ?

$\mathrm{C} 3 \mathrm{~b}$ C4b C5b 120.3(3) ?? ? ?

$\mathrm{C} 3 \mathrm{~b} \mathrm{C} 4 \mathrm{~b}$ Olb 121.7(3) ?? ? ?

$\mathrm{C} 5 \mathrm{~b}$ C4b O1b 118.0(3) ?? ? ?

C4b C5b C6b 120.3(3) ?? ? ?

$\mathrm{C} 4 \mathrm{~b}$ C5b H5b 123.8(3) ?? ? ?

$\mathrm{C} 6 \mathrm{~b}$ C5b H5b 115.7(3) ?? ? ?

$\mathrm{C} 1 \mathrm{~b}$ C6b C5b 121.1(3) ?? ? ?

C1b C6b H6b 121.8(3) ? ? ? ?

C5b C6b H6b 117.1(3) ? ? ? ?

$\mathrm{C} 4 \mathrm{~b}$ O1b H1b 112(2) ? ? ? ?

C2b' C1b' C6b' 116.7(3) ? ? ? ? 


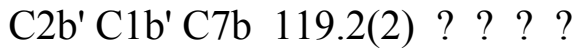
$\mathrm{C}^{\prime} \mathrm{b}^{\prime} \mathrm{C} 1 \mathrm{~b}^{\prime} \mathrm{C} 7 \mathrm{~b}$ 124.1(3) ?? ? ? $\mathrm{C} 1 b^{\prime} \mathrm{C} 2 b^{\prime} \mathrm{C} 3 b^{\prime} 122.4(3)$ ? ? ? ?

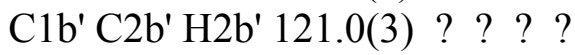
$\mathrm{C}^{2} b^{\prime} \mathrm{C} 2 \mathrm{~b}^{\prime} \mathrm{H} 2 \mathrm{~b}^{\prime}$ 116.6(3) ?? ? ? $\mathrm{C} 2 b^{\prime} \mathrm{C} 3 b^{\prime} \mathrm{C} 4 b^{\prime}$ 119.5(3) ?? ? ?

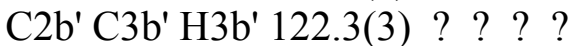
$\mathrm{C} 4 b^{\prime} \mathrm{C} 3 b^{\prime} \mathrm{H} 3 b^{\prime} 118.2(3)$ ? ? ? ?

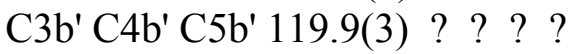
C3b' C4b' O1b' 116.9(3) ?? ? ?

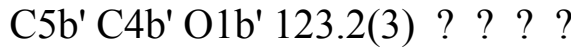
C4b' C5b' C6b' 119.5(3) ? ? ? ? C4b' C5b' H5b' 122.1(3) ?? ? ?

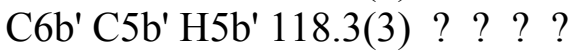

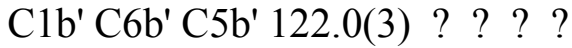
C1b' C6b' H6b' 119.8(3) ?? ? ? C5b' C6b' H6b' 118.1(3) ?? ? ? C4b' O1b' H1b' 107(2) ? ? ? ?

C1b C7b C1b' 107.9(2) ?? ? ? $\mathrm{C} 1 \mathrm{~b}$ C7b C8b 107.5(2) ?? ? ? $\mathrm{C} 1 \mathrm{~b}$ C7b C9b 112.0(2) ?? ? ? $\mathrm{C} 1 b^{\prime} \mathrm{C} 7 \mathrm{~b} \mathrm{C} 8 \mathrm{~b}$ 112.1(2) ?? ? ? $\mathrm{C} 1 b^{\prime} \mathrm{C} 7 \mathrm{~b}$ C9b 108.8(2) ?? ? ? $\mathrm{C} 8 \mathrm{~b}$ C7b C9b 108.7(3) ?? ? ? $\mathrm{C} 7 \mathrm{~b}$ C8b H8ba 107.6(3) ?? ? ? $\mathrm{C} 7 \mathrm{~b} \mathrm{C} 8 \mathrm{~b} \mathrm{H} 8 \mathrm{bb}$ 110.7(3) ?? ? ? $\mathrm{C} 7 \mathrm{~b}$ C8b H8bc 106.2(3) ?? ? ? $\mathrm{H} 8 \mathrm{ba} \mathrm{C} 8 \mathrm{~b} \mathrm{H} 8 \mathrm{bb}$ 110.6(3) ?? ? ? $\mathrm{H} 8 \mathrm{ba} \mathrm{C} 8 \mathrm{~b} \mathrm{H} 8 \mathrm{bc}$ 118.5(4)？? ? ? $\mathrm{H} 8 \mathrm{bb} \mathrm{C} 8 \mathrm{~b} \mathrm{H} 8 \mathrm{bc}$ 103.2(3) ? ? ? ? C7b C9b H9ba 113.5(3) ? ? ? ? $\mathrm{C} 7 \mathrm{~b}$ C9b H9bb 107.5(3) ?? ? ? $\mathrm{C} 7 \mathrm{~b}$ C9b H9bc 112.5(3) ?? ? ? $\mathrm{H} 9 \mathrm{ba} \mathrm{C}$ b H9bb 102.1(3) ? ? ? ? H9ba C9b H9bc 106.0(3) ? ? ? $\mathrm{H} 9 \mathrm{bb}$ C9b H9bc 115.0(4) ? ? ? $\mathrm{C} 2 \mathrm{c}$ C1c C6c 117.4(3) ?? ? ? $\mathrm{C} 2 \mathrm{c} \mathrm{C} 1 \mathrm{c} \mathrm{C} 7 \mathrm{c}$ 119.6(2) ?? ? ? $\mathrm{C} 6 \mathrm{c} \mathrm{C1c} \mathrm{C7c} \mathrm{123.0(3)} \mathrm{??} \mathrm{?} \mathrm{?}$ $\mathrm{C} 1 \mathrm{c} 2 \mathrm{c} C 3 \mathrm{c}$ 121.8(3) ?? ? ? $\mathrm{C} 1 \mathrm{c} \mathrm{C} 2 \mathrm{c} \mathrm{H} 2 \mathrm{c}$ 118.7(3) ?? ? ? $\mathrm{C} 3 \mathrm{c} \mathrm{C} 2 \mathrm{c} \mathrm{H} 2 \mathrm{c}$ 119.5(3) ?? ? ? $\mathrm{C} 2 \mathrm{c} \mathrm{C} 3 \mathrm{c} \mathrm{C} 4 \mathrm{c}$ 119.5(3) ?? ? ? $\mathrm{C} 2 \mathrm{c} \mathrm{C} 3 \mathrm{c} \mathrm{H} 3 \mathrm{c}$ 121.4(3) ?? ? ? $\mathrm{C} 4 \mathrm{c} \mathrm{C} 3 \mathrm{c} \mathrm{H} 3 \mathrm{c}$ 118.9(3) ?? ? ? 
$\mathrm{C} 3 \mathrm{c} \mathrm{C} 4 \mathrm{c} 5 \mathrm{c}$ 120.2(3) ?? ? ?

$\mathrm{C} 3 \mathrm{c} \mathrm{C} 4 \mathrm{c} \mathrm{O} 1 \mathrm{c}$ 117.1(3) ?? ? ?

$\mathrm{C} 5 \mathrm{c} \mathrm{C} 4 \mathrm{c} \mathrm{O} 1 \mathrm{c}$ 122.7(3) ?? ? ?

$\mathrm{C} 4 \mathrm{c} \mathrm{C} 5 \mathrm{c}$ C6c 120.0(3) ?? ? ?

$\mathrm{C} 4 \mathrm{c} 55 \mathrm{c} \mathrm{H} 5 \mathrm{c}$ 121.5(3) ?? ? ?

$\mathrm{C} 6 \mathrm{c} 5 \mathrm{c} \mathrm{H} 5 \mathrm{c}$ 118.4(3) ?? ? ?

$\mathrm{C} 1 \mathrm{c} 6 \mathrm{c} \mathrm{C} 5 \mathrm{c}$ 121.0(3) ?? ? ?

$\mathrm{C} 1 \mathrm{c} 66 \mathrm{cH} 6 \mathrm{c}$ 122.1(3) ?? ? ?

$\mathrm{C} 5 \mathrm{c} \mathrm{C} 6 \mathrm{c} \mathrm{H} 6 \mathrm{c}$ 117.0(3) ?? ? ?

$\mathrm{C} 4 \mathrm{c} \mathrm{O} 1 \mathrm{c} \mathrm{H} 1 \mathrm{c}$ 106(2) ? ? ? ?

$\mathrm{C} 2 \mathrm{c}^{\prime} \mathrm{C} 1 \mathrm{c}^{\prime} \mathrm{C} 6 \mathrm{c}^{\prime} 117.8(2)$ ? ? ? ?

$\mathrm{C} 2 \mathrm{c}^{\prime} \mathrm{C} 1 \mathrm{c}^{\prime} \mathrm{C} 7 \mathrm{c}$ 120.1(2) ? ? ? ?

$\mathrm{C} 6 \mathrm{c}^{\prime} \mathrm{C} 1 \mathrm{c}^{\prime} \mathrm{C} 7 \mathrm{c}$ 122.0(2) ? ? ? ?

$\mathrm{C} 1 \mathrm{c}^{\prime} \mathrm{C} 2 \mathrm{c}^{\prime} \mathrm{C} 3 \mathrm{c}^{\prime} 121.9(2)$ ? ? ? ?

$\mathrm{C} 1 \mathrm{c}^{\prime} \mathrm{C} 2 \mathrm{c}^{\prime} \mathrm{H} 2 \mathrm{c}^{\prime} 121.6(3)$ ? ? ? ?

$\mathrm{C} 3 \mathrm{c}^{\prime} \mathrm{C} 2 \mathrm{c}^{\prime} \mathrm{H} 2 \mathrm{c}^{\prime}$ 116.4(3) ? ? ? ?

$\mathrm{C} 2 \mathrm{c}^{\prime} \mathrm{C} 3 \mathrm{c}^{\prime} \mathrm{C} 4 \mathrm{c}^{\prime} 118.5(3)$ ? ? ? ?

$\mathrm{C} 2 \mathrm{c}^{\prime} \mathrm{C} 3 \mathrm{c}^{\prime} \mathrm{H} 3 \mathrm{c}^{\prime}$ 116.7(3) ? ? ? ?

$\mathrm{C} 4 \mathrm{c}^{\prime} \mathrm{C} 3 \mathrm{c}^{\prime} \mathrm{H} 3 \mathrm{c}^{\prime}$ 124.7(3) ? ? ? ?

$\mathrm{C} 3 \mathrm{c}^{\prime} \mathrm{C} 4 \mathrm{c}^{\prime} \mathrm{C} 5 \mathrm{c}^{\prime} 121.3(2)$ ? ? ? ?

$\mathrm{C} 3 \mathrm{c}^{\prime} \mathrm{C} 4 \mathrm{c}^{\prime} \mathrm{O} 1 \mathrm{c}^{\prime} 121.3(3)$ ? ? ? ?

$\mathrm{C} 5 \mathrm{c}^{\prime} \mathrm{C} 4 \mathrm{c}^{\prime} \mathrm{O} 1 \mathrm{c}^{\prime} 117.4(2)$ ? ? ? ?

$\mathrm{C} 4 \mathrm{c}^{\prime} \mathrm{C} 5 \mathrm{c}^{\prime} \mathrm{C} 6 \mathrm{c}^{\prime}$ 119.1(2) ? ? ? ?

$\mathrm{C} 4 \mathrm{c}^{\prime} \mathrm{C} 5 \mathrm{c}^{\prime} \mathrm{H} 5 \mathrm{c}^{\prime} 121.7(3)$ ? ? ? ?

$\mathrm{C} 6 \mathrm{c}^{\prime} \mathrm{C} 5 \mathrm{c}^{\prime} \mathrm{H} 5 \mathrm{c}^{\prime}$ 119.1(3) ? ? ? ?

$\mathrm{C} 1 \mathrm{c}^{\prime} \mathrm{C} 6 \mathrm{c}^{\prime} \mathrm{C} 5 \mathrm{c}^{\prime} 121.3(3)$ ? ? ? ?

$\mathrm{C} 1 \mathrm{c}^{\prime} \mathrm{C} 6 \mathrm{c}^{\prime} \mathrm{H} 6 \mathrm{c}^{\prime}$ 124.7(3) ? ? ? ?

C5c' C6c' H6c' 114.0(3) ? ? ? ?

$\mathrm{C} 4 \mathrm{c}^{\prime} \mathrm{O} 1 \mathrm{c}^{\prime} \mathrm{H} 1 \mathrm{c}^{\prime} 105(2)$ ? ? ? ?

$\mathrm{C} 1 \mathrm{c} C 7 \mathrm{c} C 1 c^{\prime}$ 109.0(2) ?? ? ?

$\mathrm{C} 1 \mathrm{c} \mathrm{C} 7 \mathrm{c} 8 \mathrm{c}$ 109.3(2) ?? ? ?

$\mathrm{C} 1 \mathrm{c} \mathrm{C} 7 \mathrm{c} C \mathrm{c}$ 112.2(2) ?? ? ?

$\mathrm{C} 1 \mathrm{c}^{\prime} \mathrm{C} 7 \mathrm{c} \mathrm{C} 8 \mathrm{c}$ 111.6(2) ?? ? ?

$\mathrm{C} 1 \mathrm{c}^{\prime} \mathrm{C} 7 \mathrm{c} \mathrm{C} 9 \mathrm{c}$ 106.9(2) ?? ? ?

$\mathrm{C} 8 \mathrm{c} \mathrm{C} 7 \mathrm{c} \mathrm{C9c} \mathrm{107.8(2)} \mathrm{??} \mathrm{?} \mathrm{?}$

$\mathrm{C} 7 \mathrm{c} \mathrm{C} 8 \mathrm{c} \mathrm{H} 8 \mathrm{ca}$ 110.6(3) ?? ? ?

$\mathrm{C} 7 \mathrm{c} \mathrm{C} 8 \mathrm{c} \mathrm{H} 8 \mathrm{cb}$ 114.7(3) ?? ? ?

$\mathrm{C} 7 \mathrm{c} \mathrm{C} 8 \mathrm{c} \mathrm{H} 8 \mathrm{cc}$ 106.8(3) ?? ? ?

$\mathrm{H} 8 \mathrm{ca} \mathrm{C} 8 \mathrm{c} \mathrm{H} 8 \mathrm{cb}$ 107.3(3) ? ? ? ?

$\mathrm{H} 8 \mathrm{ca} \mathrm{C} 8 \mathrm{c} \mathrm{H} 8 \mathrm{cc} 112.5(3)$ ? ? ? ?

$\mathrm{H} 8 \mathrm{cb} \mathrm{C} 8 \mathrm{c} \mathrm{H} 8 \mathrm{cc} 104.8(3)$ ? ? ? ?

$\mathrm{C} 7 \mathrm{c}$ C9c H9ca 111.2(3) ? ? ? ?

$\mathrm{C} 7 \mathrm{c} \mathrm{C} 9 \mathrm{c} \mathrm{H} 9 \mathrm{cb}$ 111.5(3) ?? ? ?

$\mathrm{C} 7 \mathrm{c}$ C9c H9cc 115.2(3) ?? ? ?

$\mathrm{H} 9 \mathrm{ca} \mathrm{C9c} \mathrm{H9cb} \mathrm{111.6(3)？？？}$ 
$\mathrm{H} 9 \mathrm{ca} \mathrm{C} 9 \mathrm{c} \mathrm{H} 9 \mathrm{cc}$ 100.5(3) ? ? ? ?

$\mathrm{H} 9 \mathrm{cb} \mathrm{C9c} \mathrm{H9cc} \mathrm{106.4(3)} \mathrm{?} \mathrm{?} \mathrm{?}$

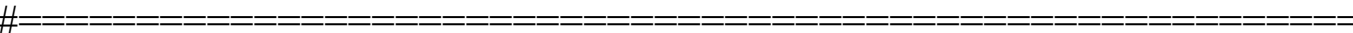

$=$

\# Additional structures (sections 5-10 and associated data_? identifiers)

\# may be added at this point.

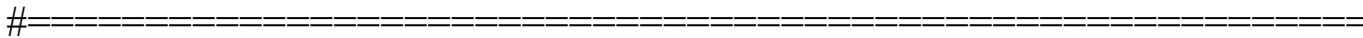

$==\mathrm{=}=\mathrm{=}=\mathrm{=}=\mathrm{=}=\mathrm{=}=\mathrm{=}=$

\# The following lines are used to test the character set of files sent by

\# network email or other means. They are not part of the CIF data set. 